\title{
A Ligand Exchange Process for the Diversification of Palladium Oxidative Addition Complexes
}

\author{
Ryan P. King, ${ }^{1}$ Shane W. Krska, ${ }^{2}$ and Stephen L. Buchwald ${ }^{1 *}$ \\ ${ }^{1}$ Department of Chemistry, Massachusetts Institute of Technology, Cambridge, Massachusetts \\ 02139, United States \\ ${ }^{2}$ Merck \& Co., Inc., Kenilworth, New Jersey 07033, United States \\ *Correspondence to: sbuchwal@mit.edu
}

Supporting Information 


\section{Table of Contents}

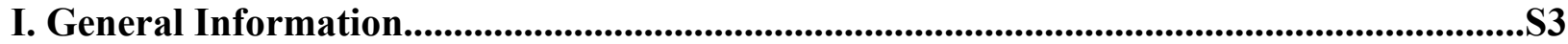

II. Synthesis of Pd OAC Starting Material..............................................................................S9

III. Pd OAC Ligand Exchange Reactions.....................................................................S18

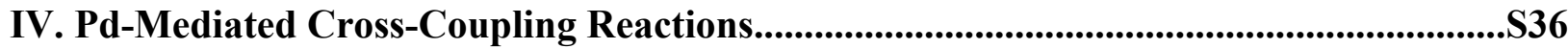

V. Pd-Catalyzed Cross-Coupling Reactions...............................................................................S39

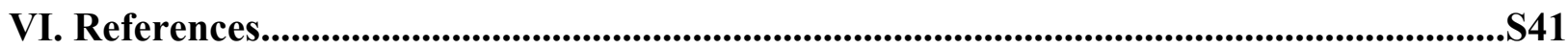

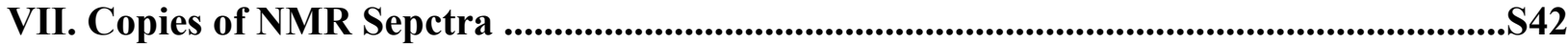




\section{General Information}

\section{General Analytical Information}

All compounds were characterized by ${ }^{1} \mathrm{H}$ NMR, ${ }^{13} \mathrm{C}$ NMR, ${ }^{19} \mathrm{~F}$ NMR (where applicable), ${ }^{31} \mathrm{P}$ NMR (where applicable), and Infrared (IR) spectroscopy, and melting point analysis. All new compounds were also characterized by either elemental analysis (EA) or high-resolution mass spectrometry (HRMS). Nuclear Magnetic Resonance spectra were recorded on a Bruker 400, 500 , or $600 \mathrm{MHz}$ instrument. All NMR spectra are recorded in $\delta$ units and parts per million (ppm). The multiplicities are abbreviated with s (singlet), br s (broad singlet), $d$ (doublet), $t$ (triplet), q (quartet), and hept (heptet). All ${ }^{1} \mathrm{H}$ and ${ }^{13} \mathrm{C}$ spectra were calibrated using residual solvent as an internal reference $\left(\mathrm{CDCl}_{3}: \delta 7.26 \mathrm{ppm}\right.$ and $\delta 77.16 \mathrm{ppm}$ respectively; $\mathrm{CD}_{2} \mathrm{Cl}_{2}: \delta$ $5.32 \mathrm{ppm}$ and $\delta 53.84 \mathrm{ppm}$ respectively). ${ }^{1}$ All ${ }^{19} \mathrm{~F}$ NMR spectra were calibrated to an external standard of trifluorotoluene $\left(\mathrm{PhCF}_{3}\right)$ in $\mathrm{CDCl}_{3}(\delta-63.72 \mathrm{ppm})$. All ${ }^{31} \mathrm{P} \mathrm{NMR}$ spectra were calibrated to an external standard of triphenylphosphine in $\mathrm{CDCl}_{3}(\delta-4.90 \mathrm{ppm})$. All ${ }^{13} \mathrm{C} \mathrm{NMR}$ and ${ }^{31} \mathrm{P}$ NMR spectra were obtained with ${ }^{1} \mathrm{H}$ NMR decoupling. Elemental analyses were performed by Atlantic Microlabs Inc., Norcross, GA, USA. All HRMS were recorded on an Agilent Technologies 6545 Q-TOF LC/MS system. All IR spectra were obtained on a Thermo Scientific Nicolet iS5 spectrometer (iD5 ATR, diamond) and were reported in wavenumbers $\left(\mathrm{cm}^{-1}\right)$. Melting points were obtained using a Stanford Research Systems EZ-melt melting point apparatus. All melting points herein described as decomposed (dec.) were compounds that turned black and melted over the reported range. LC/MS analysis was performed with a Thermo Scientific Accucore C18 column ( $30 \times 2.1 \mathrm{~mm}, 2.6 \mu \mathrm{m}$ particle size) maintained at $45{ }^{\circ} \mathrm{C}$ within an instrument consisting of Agilent 1260 series binary pump and an Agilent 6120 quadrupole MS operating in positive MM-ES+APCI ionization mode. Flash column chromatography was performed using SiliCycle SiliaFlash ${ }^{\circledR}$ F60 silica gel $(40-63 \mu \mathrm{m}, 230-400$ mesh, $60 \AA$ pore diameter). Preparatory thin-layer chromatography was performed using SiliCycle glass-backed plates pre-coated with silica gel $(1000 \mu \mathrm{m}, 60 \AA$ pore diameter $)$ impregnated with a fluorescent indicator $(254 \mathrm{~nm})$.

\section{General Reagent Information}

Reagent information: All reagents were purchased from commercial sources and used as received unless otherwise noted. 4,4'-Di-tert-butyl-2,2'-dipyridyl (dtbbpy), potassium phosphate monohydrate, tribasic $\left(\mathrm{K}_{3} \mathrm{PO}_{4}-\mathrm{H}_{2} \mathrm{O}\right)$, silver methanesulfonate (AgOMs), trimethylphosphite $\left[\mathrm{P}(\mathrm{OMe})_{3}\right]$, and trimethylphosphine $\left(\mathrm{PMe}_{3}, 1.0 \mathrm{M}\right.$ in toluene, Sure-Seal $\left.{ }^{\mathrm{TM}}\right)$ were purchased from Millipore-Sigma. Informer compounds X4 and X14 were obtained from Merck \& Co., Inc. Rivaroxaban was purchased from Combi-Blocks. Indometacin was purchased from Acros. Triphenylphosphite $\left[\mathrm{P}(\mathrm{OPh})_{3}\right]$ was purchased from Tokyo Chemical Industry. SilaMetS DMT resin was purchased from SiliCycle. Deuterated solvents were purchased from Cambridge Isotope Laboratories and dried over activated molecular sieves ( $4 \AA$ ) overnight prior to use. XantPhos and JosiPhos SL-J009-1 were purchased from Strem. Tert-ButylXPhos ( $t$-BuXPhos), XPhos, SPhos, RuPhos, tert-ButylBrettPhos ( $t$-BuBrettPhos), and BrettPhos were gifts from Millipore-Sigma, for which we are grateful. Sodium tert-butoxide $(\mathrm{NaO} t$-Bu) was purchased from Strem and stored in a $\mathrm{N}_{2}$-filled glovebox. Portions were removed from the glovebox in an oven-dried $20 \mathrm{~mL}$ scintillation vial, stored in a desiccator box, and used within 3 days. 
Solvent information: Pentane (reagent grade, SKU: 158941), methylene chloride $\left(\mathrm{CH}_{2} \mathrm{Cl}_{2}, \mathrm{HPLC}\right.$ grade, stabilized, SKU: 34856), methanol (MeOH, HPLC grade), ethyl acetate (EtOAc, HPLC grade), and diethyl ether $\left(\mathrm{Et}_{2} \mathrm{O}\right.$, stabilized, SKU: 673811) for purifications were purchased from Millipore-Sigma. $n$-Hexane (anhydrous, Sure-Seal ${ }^{\mathrm{TM}}$, SKU: 296090), cyclohexane (anhydrous, Sure-SealTM, SKU: 227048), and 1,4-dioxane (anhydrous, Sure-SealTM, SKU: 296309) for reactions were purchased from Millipore-Sigma and degassed prior to use by placing under vacuum for $10 \mathrm{~s}$, sonicating, and replenishing with an atmosphere of nitrogen gas. This cycle was repeated 3 times. Pentane (anhydrous, Sure-SealTM, SKU: 236705) for reactions was purchased from Millipore-Sigma and degassed by sparging with argon for $30 \mathrm{~min}$ prior to use. Tetrahydrofuran (THF, anhydrous) and dichloromethane (anhydrous) for reactions were purchased from J.T. Baker in CYCLE-TAINER ${ }^{\circledR}$ delivery kegs and purified by passing through two packed columns of neutral alumina and copper(II) oxide successively under an argon atmosphere prior to use. $\begin{array}{lcccc}(\mathrm{cod}) \mathrm{Pd}\left(\mathrm{CH}_{2} \mathrm{TMS}\right)_{2},{ }^{2} & {[(t \text {-BuXPhos }) \mathrm{Pd}]_{2}(\mathrm{cod}),{ }^{3}} & {[(t \text {-BuBrettPhos }) \mathrm{Pd}]_{2}(\mathrm{cod}),{ }^{3},} & (t \text { - } \\ \text { BuXPhos }) \mathrm{Pd}(\text { rivaroxaban }) \mathrm{Cl},{ }^{2} & (t \text {-BuXPhos }) \mathrm{Pd}(\mathrm{X} 4) \mathrm{Br},{ }^{2} & (t \text {-BuXPhos }) \mathrm{Pd}(\mathrm{Ph}) \mathrm{Cl},{ }^{2} & 4-\end{array}$ (trifluoromethyl)phenyl trifluoromethanesulfonate, ${ }^{4}$ CPhos, ${ }^{5} \mathrm{PhXPhos},{ }^{6} t$-BuXPhos Pd G4, ${ }^{7}$ RuPhos Pd G4, and XPhos Pd G4 ${ }^{7}$ were prepared according to literature procedures

\section{General Reaction Information}

-For $\mathrm{CH}_{2} \mathrm{Cl}_{2}$ and pentane, both solvents stored under an inert atmosphere (nitrogen or argon, and degassed, see above) and stored in air were used. In specific procedures, $\mathrm{CH}_{2} \mathrm{Cl}_{2}$ and pentane stored under an inert atmosphere are denoted as (degassed).

-Glassware denoted as oven-dried was placed in an oven at $140{ }^{\circ} \mathrm{C}$ for at least $16 \mathrm{~h}$ and then allowed to cool to room temperature under vacuum prior to use.

-Unless otherwise noted, all products were dried in vacuo on a Schlenk line at room temperature overnight $(\sim 16 \mathrm{~h})$.

-The components for reactions involving $8 \mathrm{~mL}, 16 \mathrm{~mL}, 24 \mathrm{~mL}$, and $36 \mathrm{~mL}$ reaction tubes are described below. 


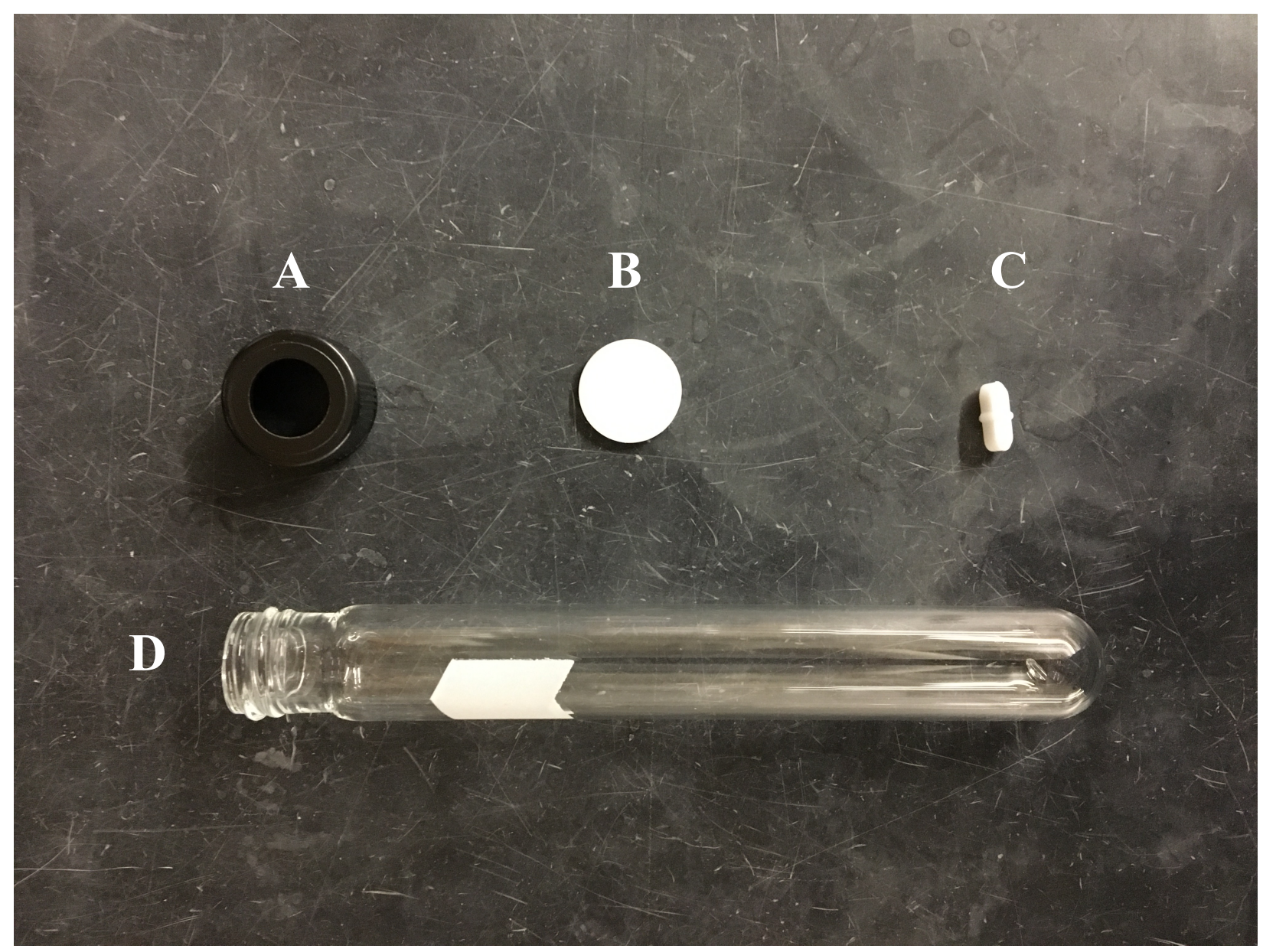

Figure S1: $8 \mathrm{~mL}$ reaction tube components

A. Thermo Scientific 13-425 phenolic cap (part \# C4014-66)

B. Thermo Scientific PTFE-lined silicone septum (part \# C45015-60)

C. A small PTFE-coated magnetic stir bar

D. Fisher Scientific 13 x $100 \mathrm{~mm}$ (8 mL) disposable culture tube S/C (part \# 1495935C) 


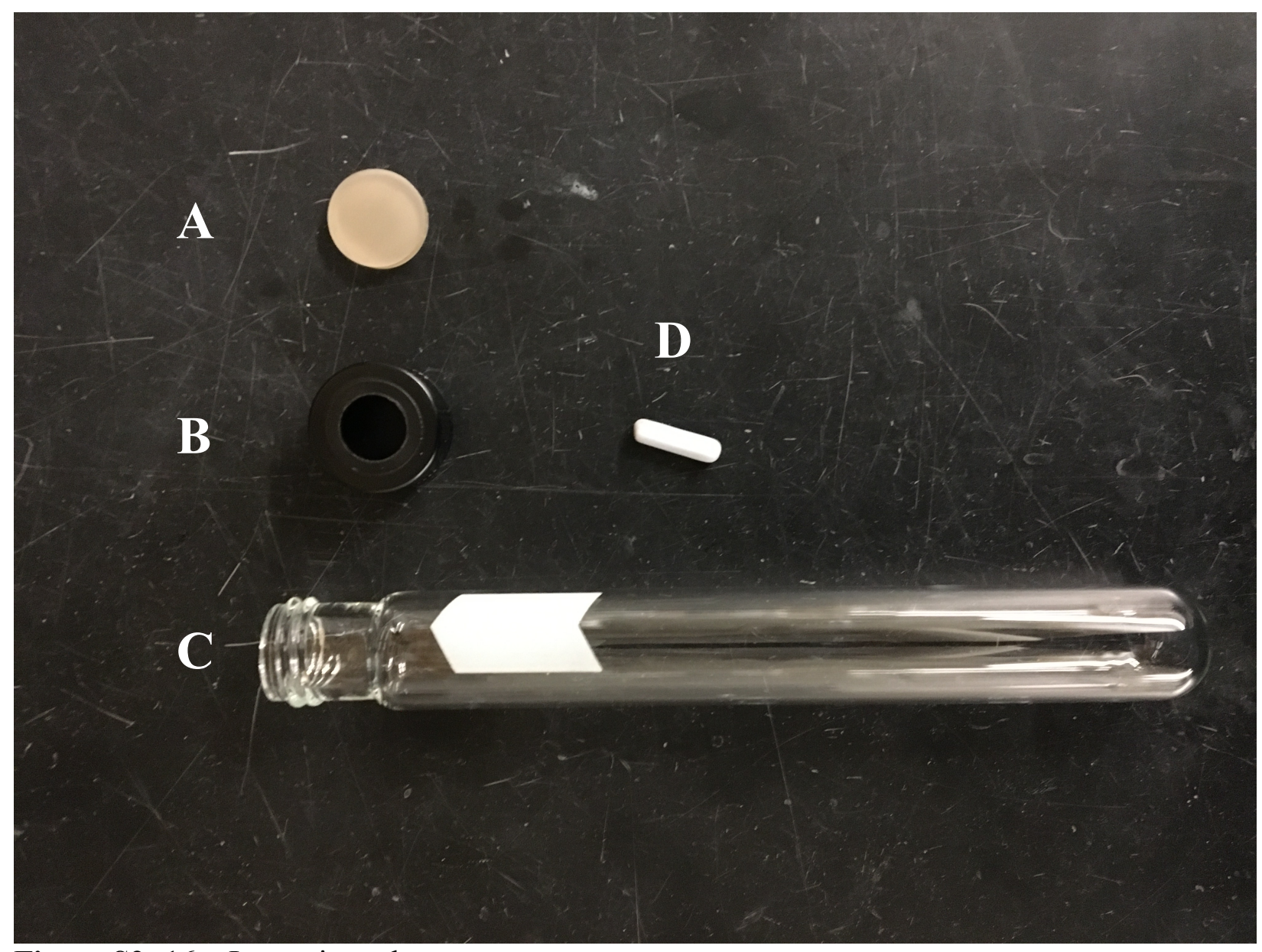

Figure S2: $16 \mathrm{~mL}$ reaction tube components

A. Thermo Scientific PTFE-lined silicone septum (part \# B7995-15)

B. Kimble Chase 15-425 phenolic open top S/T cap (cat. \# 73804-15425)

C. Fisher Scientific 16 x $125 \mathrm{~mm}$ (16 mL) disposable culture tube S/C (part \# 1496226G)

D. A small PTFE-coated magnetic stir bar 


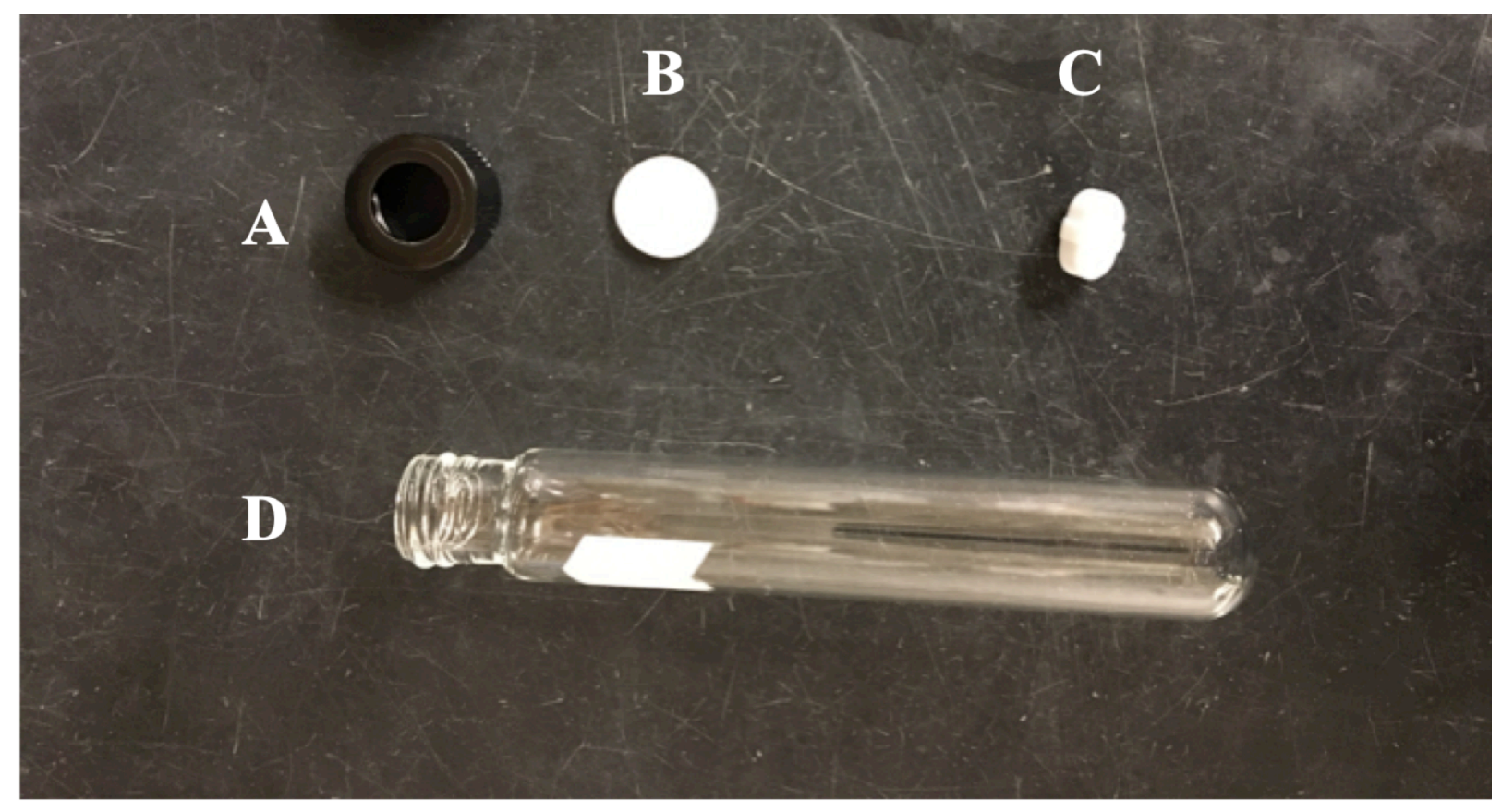

Figure S3: $24 \mathrm{~mL}$ reaction tube components

A. Kimble Chase 18-400 phenolic open top S/T cap (cat. \# 73804-18400). Henceforth referred to as a septum cap (when applicable)

B. Thermo Scientific PTFE-lined silicone septum (part \# C45015-60)

C. A small PTFE-coated magnetic stir bar

D. Fisher Scientific 20 x $125 \mathrm{~mm}(24 \mathrm{~mL})$ disposable culture tube S/C (part \# 1495937A) 


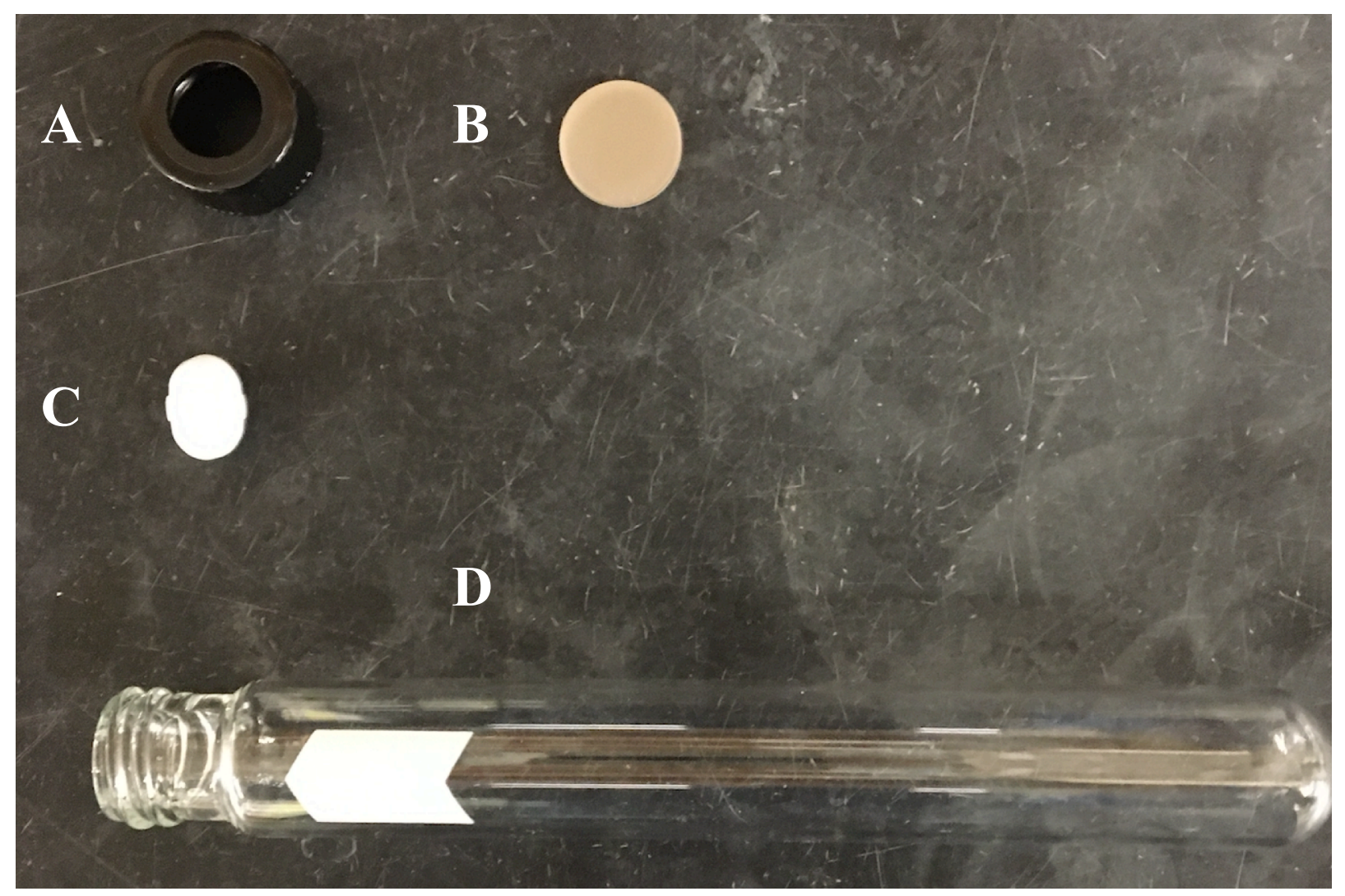

Figure S4: $36 \mathrm{~mL}$ reaction tube components

A. Kimble Chase 18-400 phenolic open top S/T cap (cat. \# 73804-18400). Henceforth referred to as a septum cap (when applicable)

B. Thermo Scientific PTFE-lined silicone septum (part \# C45015-60)

C. A small PTFE-coated magnetic stir bar

D. Fisher Scientific 20 x 150 mm (36 mL) disposable culture tube S/C (part \# 1495937C) 


\section{Synthesis of Palladium OAC Starting Material}

\section{General Procedure A: Synthesis of OACs from (cod)Pd(CH2 $\left(\mathrm{CHS}_{2}\right.$}

An oven-dried $36 \mathrm{~mL}$ reaction tube was equipped, while still hot, with a magnetic stir bar and septum cap. The tube was allowed to cool to room temperature under vacuum by piercing the septum with a needle connected to a dual manifold by a rubber hose. At this point, the cap was removed and the tube was charged with ligand (1.00 mmol, 1.0 equiv). The tube was brought into a nitrogen-filled glovebox where (cod)Pd( $\left.\mathrm{CH}_{2} \mathrm{TMS}\right)_{2}$ (389 mg, $1.00 \mathrm{mmol}, 1.0$ equiv), aryl halide (1.50 mmol, 1.5 equiv), and pentane (degassed, $10 \mathrm{~mL}$ ) were subsequently added. The tube was capped, removed from the glovebox, and placed under nitrogen by piercing the septum with a needle connected to the dual manifold by a rubber hose. The reaction mixture was allowed to stir at room temperature overnight $(\sim 16 \mathrm{~h})$. Over the course of the reaction, the mixture became homogeneous and subsequently the corresponding OAC precipitated. At this time, the reaction tube was opened to air and the reaction mixture was diluted with pentane $(5 \mathrm{~mL})$ to assist with precipitation. The precipitate was collected on a fritted funnel, washed with pentane $(20 \mathrm{~mL})$, and dried in vacuo on a Schlenk line overnight $(\sim 16 \mathrm{~h})$ to afford the desired complex.

\section{General Procedure B: Synthesis of OACs from $(\mathrm{L} \cdot \mathrm{Pd})_{2}(\operatorname{cod})$}

An oven-dried $24 \mathrm{~mL}$ reaction tube was equipped, while still hot, with a magnetic stir bar and septum cap. The tube was allowed to cool to room temperature under vacuum by piercing the septum with a needle connected to a dual manifold by a rubber hose. At this point, the cap was removed and the tube was charged with aryl halide $(0.500 \mathrm{mmol}, 1.0$ equiv). The tube was brought into a nitrogen-filled glovebox where $(\mathrm{L} \cdot \mathrm{Pd})_{2}(\mathrm{cod})(0.250 \mathrm{mmol}, 0.5$ equiv, 1.0 equiv $[\mathrm{Pd}])$, and THF $(5 \mathrm{~mL})$ were subsequently added. The tube was capped, removed from the glovebox, and placed under nitrogen by piercing the septum with a needle connected to the dual manifold by a rubber hose. The reaction mixture was allowed to stir at room temperature for $1 \mathrm{~h}$. After $1 \mathrm{~h}$, the reaction tube was opened to air and the reaction mixture was pipetted into a 100 $\mathrm{mL}$ beaker containing $50 \mathrm{~mL}$ pentane dropwise to precipitate the OAC. The precipitate was collected on a fritted funnel, washed with pentane $(50 \mathrm{~mL})$, and dried in vacuo on a Schlenk line overnight $(\sim 16 \mathrm{~h})$ to afford the desired complex.

\section{(t-BuXPhos)Pd[(4-CF 3$) \mathrm{Ph}] \mathrm{Cl}(\mathrm{S} 1)$}

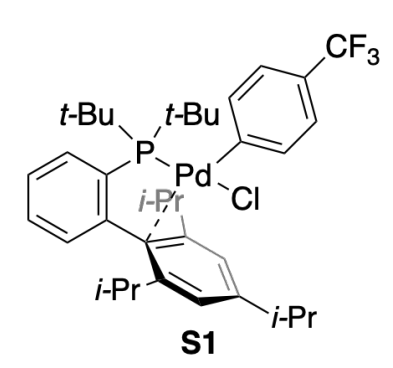

Compound S1 was prepared according to the General Procedure A using (cod) $\mathrm{Pd}\left(\mathrm{CH}_{2} \mathrm{TMS}\right)_{2}$ (389 mg, $1.00 \mathrm{mmol}, 1$ equiv), $t$-BuXPhos (425 mg, $1.00 \mathrm{mmol}, 1.0$ equiv), 4-chlorobenzotrifluoride (200 $\mu \mathrm{L}$, $1.50 \mathrm{mmol}, 1.5$ equiv), and pentane (degassed, $10.0 \mathrm{~mL}$ ). The precipitate was collected on a fritted funnel to yield S1 $(554 \mathrm{mg}$, $78 \%$ ) as a pale-yellow powder.

${ }^{1} \mathbf{H}$ NMR $\left(600 \mathrm{MHz}, \mathrm{CD}_{2} \mathrm{Cl}_{2}\right) \delta 7.99(\mathrm{td}, J=6.4,3.3 \mathrm{~Hz}, 1 \mathrm{H}), 7.45-$ $7.39(\mathrm{~m}, 2 \mathrm{H}), 7.30(\mathrm{~d}, J=7.3 \mathrm{~Hz}, 2 \mathrm{H}), 7.10$ (s, 2H), 7.08 (d, $J=8.2$ 
$\mathrm{Hz}, 2 \mathrm{H}), 6.82-6.77$ (m, 1H), 3.02 (hept, $J=6.9 \mathrm{~Hz}, 1 \mathrm{H}), 2.55$ (hept, $J=6.7 \mathrm{~Hz}, 2 \mathrm{H}), 1.59$ (d, $J$ $=6.8 \mathrm{~Hz}, 6 \mathrm{H}), 1.41(\mathrm{~s}, 9 \mathrm{H}), 1.38(\mathrm{~s}, 9 \mathrm{H}), 1.36(\mathrm{~d}, J=7.0 \mathrm{~Hz}, 6 \mathrm{H}), 0.91(\mathrm{~d}, J=6.7 \mathrm{~Hz}, 6 \mathrm{H})$.

${ }^{13} \mathrm{C}$ NMR $\left(151 \mathrm{MHz}, \mathrm{CD}_{2} \mathrm{Cl}_{2}\right) \delta 158.0,153.0,147.7,147.6,143.7,138.9,138.9,136.6,136.4$, $135.6,134.8,134.7,130.7,130.6,128.0,126.3,126.3,126.2,125.6,125.4,125.1,125.1,124.9$, $124.5,124.5,124.4,122.6,122.2,122.2,122.2,122.2,39.8,39.7,35.0,31.8,31.8,31.7,25.6$, 24.9, 24.8 .

${ }^{19}$ F NMR $\left(565 \mathrm{MHz}, \mathrm{CD}_{2} \mathrm{Cl}_{2}\right) \delta-63.05$.

${ }^{31} \mathbf{P}$ NMR $\left(202 \mathrm{MHz}, \mathrm{CD}_{2} \mathrm{Cl}_{2}\right) \delta 53.86$.

IR (neat): 3065, 2965, 2927, 2901, 2866, 1604, 1585, 1559, 1476, 1459, 1426, 1400, 1383, 1360, $1320,1267,1251,1151,1104,1095,1064,1016,1007,938,870,825,813,765,758,749$, 735, $718,675,645,595 \mathrm{~cm}^{-1}$

M.P.: $155-157^{\circ} \mathrm{C}($ dec.)

EA Calcd. for $\mathrm{C}_{36} \mathrm{H}_{49} \mathrm{ClF}_{3} \mathrm{PPd}$ : C, 60.76; H, 6.94. Found: C, 60.57; H, 6.98.

\section{$(t$-BuXPhos)Pd[(4-CF $) \operatorname{Ph}] \mathrm{Br}(\mathrm{S} 2)$}

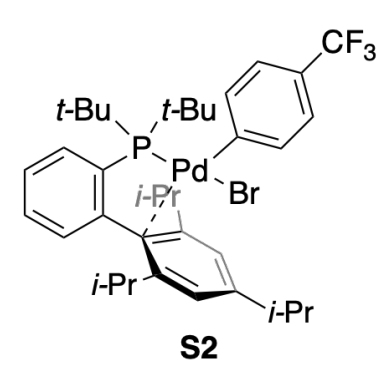

Compound $\mathbf{S 2}$ was prepared according to the General Procedure $\mathbf{A}$ using (cod) $\mathrm{Pd}\left(\mathrm{CH}_{2} \mathrm{TMS}\right)_{2}$ (389 mg, $1.00 \mathrm{mmol}, 1$ equiv), $t$-BuXPhos (425 mg, $1.00 \mathrm{mmol}, 1.0$ equiv), 4-bromobenzotrifluoride $(210 \mu \mathrm{L}$, $1.50 \mathrm{mmol}, 1.5$ equiv), and pentane (degassed, $10.0 \mathrm{~mL}$ ). The precipitate was collected on a fritted funnel to yield $\mathbf{S 2}(659 \mathrm{mg}$, $87 \%$ ) as a yellow powder.

${ }^{1} \mathbf{H}$ NMR $\left(500 \mathrm{MHz}, \mathrm{CD}_{2} \mathrm{Cl}_{2}\right) \delta 8.03-7.96(\mathrm{~m}, 1 \mathrm{H}), 7.45-7.37(\mathrm{~m}$, $2 \mathrm{H}), 7.30(\mathrm{~d}, J=7.5 \mathrm{~Hz}, 2 \mathrm{H}), 7.11(\mathrm{~s}, 2 \mathrm{H}), 7.07(\mathrm{~d}, J=8.2 \mathrm{~Hz}, 2 \mathrm{H})$,

6.76 (ddd, $J=5.1,3.8,2.7 \mathrm{~Hz}, 1 \mathrm{H}$ ), 3.07 (hept, $J=6.9 \mathrm{~Hz}, 1 \mathrm{H}$ ), 2.55 (hept, $J=6.7 \mathrm{~Hz}, 2 \mathrm{H}$ ), 1.59 $(\mathrm{d}, J=6.8 \mathrm{~Hz}, 6 \mathrm{H}), 1.40(\mathrm{~s}, 9 \mathrm{H}), 1.40-1.37(\mathrm{~m}, 15 \mathrm{H}), 0.91(\mathrm{~d}, J=6.6 \mathrm{~Hz}, 6 \mathrm{H})$.

${ }^{13}$ C NMR $\left(126 \mathrm{MHz}, \mathrm{CD}_{2} \mathrm{Cl}_{2}\right) \delta 157.9,152.9,147.4,147.3,139.8,139.7,139.7,139.6,136.4$, $136.2,135.7,135.0,134.9,130.7,130.6,129.0,126.3,126.2,126.2,125.4,125.3,125.1,124.9$, $124.9,124.2,122.0,122.0,121.9,121.9,40.1,40.0,34.8,31.8,31.8,31.7,25.6,24.9,24.8$.

${ }^{19}$ F NMR $\left(471 \mathrm{MHz}, \mathrm{CD}_{2} \mathrm{Cl}_{2}\right) \delta-63.03$.

${ }^{31} \mathbf{P}$ NMR $\left(203 \mathrm{MHz}, \mathrm{CD}_{2} \mathrm{Cl}_{2}\right) \delta 51.40$.

IR (neat): 3058, 3008, 2968, 2951, 2925, 2901, 2866, 1601, 1585, 1554, 1478, 1459, 1419, 1383, 1360, 1324, 1251, 1220, 1151, 1116, 1097, 1069, 1050, 1019, 1005, 934, 872, 829, 815, 782, $768,756,732,718,678,645,593 \mathrm{~cm}^{-1}$

M.P.: $163-165^{\circ} \mathrm{C}(\mathrm{dec}$.

HRMS (ESI) Calcd. For $\mathrm{C}_{36} \mathrm{H}_{49} \mathrm{~F}_{3} \mathrm{PPd}(\mathrm{M}-\mathrm{Br})^{+}$: 675.2553. Found: 675.2569.

\section{(t-BuXPhos)Pd[(4-CF 3$) P h] O T f(S 3)$}

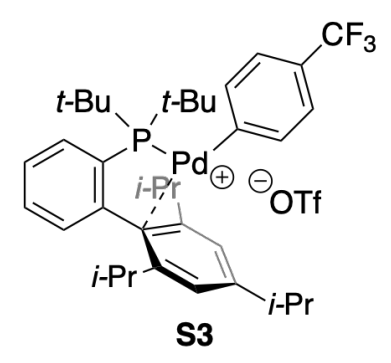

Compound $\mathbf{S 3}$ was prepared according to the General Procedure $\mathbf{A}$ using (cod) $\mathrm{Pd}\left(\mathrm{CH}_{2} \mathrm{TMS}\right)_{2}$ (389 mg, $1.00 \mathrm{mmol}, 1$ equiv), $t$-BuXPhos (425 mg, $1.00 \mathrm{mmol}, 1.0$ equiv), 4-(trifluoromethyl)phenyl trifluoromethanesulfonate (441 $\mathrm{mg}, 1.50 \mathrm{mmol}, 1.5 \mathrm{equiv})$, and pentane (degassed, $10.0 \mathrm{~mL}$ ). The precipitate was collected on a fritted funnel to yield $\mathbf{S 3}$ (712 $\mathrm{mg}, 86 \%$ ) as a yellow powder.

${ }^{1} \mathbf{H}$ NMR $\left(600 \mathrm{MHz}, \mathrm{CD}_{2} \mathrm{Cl}_{2}\right) \delta 7.93(\mathrm{t}, J=7.9 \mathrm{~Hz}, 1 \mathrm{H}), 7.85-7.79$ 
(m, 2H), 7.73 (ddt, $J=8.5,6.3,2.2 \mathrm{~Hz}, 1 \mathrm{H}), 7.40$ (dd, $J=8.1,3.6 \mathrm{~Hz}, 2 \mathrm{H}), 7.31$ (s, 2H), 7.26 (d, $J=8.0 \mathrm{~Hz}, 2 \mathrm{H}), 2.58-2.46(\mathrm{~m}, 3 \mathrm{H}), 1.48(\mathrm{~d}, J=6.7 \mathrm{~Hz}, 6 \mathrm{H}), 1.36(\mathrm{~s}, 9 \mathrm{H}), 1.33$ (s, 9H), 1.18 (d, $J=6.8 \mathrm{~Hz}, 6 \mathrm{H}), 0.93(\mathrm{~d}, J=7.0 \mathrm{~Hz}, 6 \mathrm{H})$.

${ }^{13}$ C NMR (151 MHz, $\left.\mathrm{CD}_{2} \mathrm{Cl}_{2}\right) \delta 151.1,146.9,146.9,143.5,143.4,141.8,141.7,139.8,139.7$, $139.7,139.5,136.1,132.5,132.5,131.6,131.6,131.6,131.6,129.2,129.2,129.1,128.9,128.7$, $128.5,127.3,125.5,124.6,124.6,124.6,124.6,124.5,123.7,122.5,121.9,121.2,121.1,120.4$, $118.2,114.8,40.9,40.7,33.9,31.7,30.8,30.8,26.5,22.3,21.4$.

${ }^{19}$ F NMR $\left(565 \mathrm{MHz}, \mathrm{CD}_{2} \mathrm{Cl}_{2}\right) \delta-63.68,-79.79$.

${ }^{31} \mathbf{P}$ NMR $\left(203 \mathrm{MHz}, \mathrm{CD}_{2} \mathrm{Cl}_{2}\right) \delta 96.29$.

IR (neat): 3065, 2975, 2930, 2882, 1585, 1464, 1417, 1393, 1369, 1324, 1275, 1258, 1222, 1149 , $1118,1097,1066,1028,1002,926,881,839,820,782,770,763,751,723,638 \mathrm{~cm}^{-1}$

M.P.: $215-218{ }^{\circ} \mathrm{C}(\mathrm{dec}$.)

EA Calcd. for $\mathrm{C}_{37} \mathrm{H}_{49} \mathrm{~F}_{3} \mathrm{O}_{3}$ PPdS: C, 53.85; H, 5.99. Found: C, 53.61; H, 6.11 .

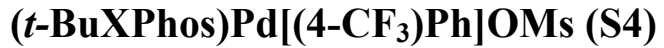

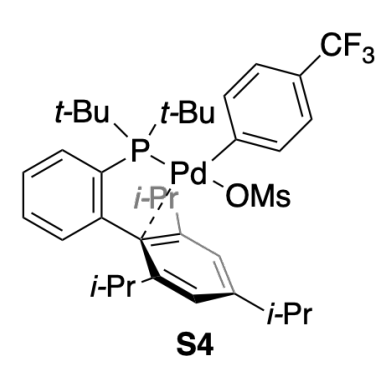

An oven-dried $24 \mathrm{~mL}$ reaction tube was equipped, while still hot, with a magnetic stir bar and septum cap. The tube was allowed to cool to room temperature under vacuum by piercing the septum with a needle connected to a dual manifold by a rubber hose. At this point the cap was removed and the tube was charged with $\mathbf{S 2}(378 \mathrm{mg}$, $0.500 \mathrm{mmol}$ ) and AgOMs (122 $\mathrm{mg}, 0.600 \mathrm{mmol}, 1.2$ equiv). The tube was recapped and covered in aluminum foil. The septum was pierced with a needle connected to the double manifold by a rubber hose, and the tube was evacuated and backfilled with nitrogen (the evacuation/backfill process was repeated a total of three times). Under nitrogen, $\mathrm{CH}_{2} \mathrm{Cl}_{2}$ (degassed, $2.5 \mathrm{~mL}$ ) was added to the tube via syringe. The reaction mixture was allowed to stir at room temperature for $2 \mathrm{~h}$. At this time, the reaction tube was opened to air and the reaction mixture was filtered through a $\sim 1 \mathrm{~cm}$ plug of Celite ${ }^{\mathbb{B}}$ into a $100 \mathrm{~mL}$ round-bottom flask. The Celite ${ }^{\circledR}$ plug was further washed with $\mathrm{CH}_{2} \mathrm{Cl}_{2}(25 \mathrm{~mL})$. The combined yellow-colored filtrate was concentrated in vacuo with the assistance of a rotary evaporator. The resulting residue was suspended in pentane $(25 \mathrm{~mL})$ and the suspension was triturated with the assistance of sonication to yield a yellow powder. The powder was collected on a fritted funnel, washed with pentane (25 $\mathrm{mL})$, and dried in vacuo on a Schlenk line overnight $(\sim 16 \mathrm{~h})$ to yield $\mathbf{S 4}(335 \mathrm{mg}, 87 \%)$ as a yellow powder.

${ }^{1}$ H NMR $\left(600 \mathrm{MHz}, \mathrm{CD}_{2} \mathrm{Cl}_{2}\right) \delta 8.00-7.94(\mathrm{~m}, 1 \mathrm{H}), 7.56-7.47(\mathrm{~m}, 4 \mathrm{H}), 7.26(\mathrm{~s}, 2 \mathrm{H}), 7.19(\mathrm{~d}$, $J=8.2 \mathrm{~Hz}, 2 \mathrm{H}), 7.07$ (dd, $J=8.0,3.3 \mathrm{~Hz}, 1 \mathrm{H}), 3.01$ (hept, $J=7.0 \mathrm{~Hz}, 1 \mathrm{H}), 2.53$ (h, $J=6.7 \mathrm{~Hz}$, 2H), $1.64(\operatorname{app~d}, J=6.8 \mathrm{~Hz}, 9 \mathrm{H}), 1.41$ (s, 9H), $1.38(\mathrm{~s}, 9 \mathrm{H}), 1.23(\mathrm{~d}, J=7.0 \mathrm{~Hz}, 6 \mathrm{H}), 0.97$ (d, $J$ $=6.7 \mathrm{~Hz}, 6 \mathrm{H})$.

${ }^{13}$ C NMR $\left(151 \mathrm{MHz}, \mathrm{CD}_{2} \mathrm{Cl}_{2}\right) \delta 155.8,152.5,146.1,146.0,143.9,143.85,138.81,138.79$, 135.98, 135.78, 135.38, 133.83, 133.76, 131.2, 131.2, 127.6, 127.5, 127.3, 127.1, 127.0, 127.0, $126.9,125.8,124.0,123.2,123.1,123.1,123.1,122.9,122.9,122.2,40.4,40.3,39.5,34.0,31.9$, $31.5,31.5,26.2,26.2,24.0,23.2,23.1$.

${ }^{19}$ F NMR $\left(565 \mathrm{MHz}, \mathrm{CD}_{2} \mathrm{Cl}_{2}\right) \delta-63.41$.

${ }^{31} \mathbf{P}$ NMR $\left(202 \mathrm{MHz}, \mathrm{CD}_{2} \mathrm{Cl}_{2}\right) \delta 66.79$. 
IR (neat): 3050, 2972, 2925, 2901, 2866, 1604, 1580, 1559, 1537, 1476, 1455, 1426, 1400, 1386, $1360,1329,1320,1258,1182,1159,1142,1102,1095,1040,1007,998,953,936,926,891$, $874,822,808,770,756,747,735,723,675,649,595 \mathrm{~cm}^{-1}$

M.P.: $154-159^{\circ} \mathrm{C}(\mathrm{dec}$.

HRMS (ESI) Calcd. For $\mathrm{C}_{36} \mathrm{H}_{49} \mathrm{~F}_{3} \mathrm{PPd}(\mathrm{M}-\mathrm{OMs})^{+}:$675.2553. Found: 675.2564.

\section{(t-BuXPhos)Pd[(2-Me-4-CF 3$) P h] B r(S 5)$}

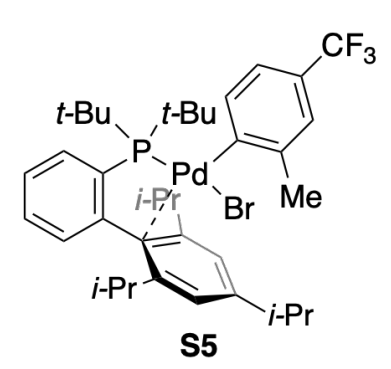

Compound S5 was prepared according to the General Procedure A using (cod) $\mathrm{Pd}\left(\mathrm{CH}_{2} \mathrm{TMS}\right)_{2}$ (389 mg, $1.00 \mathrm{mmol}, 1$ equiv), $t$-BuXPhos (425 mg, 1.00 mmol, 1.00 equiv), 1-bromo-2-methyl-4(trifluoromethyl)benzene (359 $\mathrm{mg}, 1.50 \mathrm{mmol}, 1.5$ equiv), and pentane (degassed, $10.0 \mathrm{~mL}$ ). The precipitate was collected on a fritted funnel to yield $\mathbf{S 5}$ (608 $\mathrm{mg}, 79 \%)$ as a pale-yellow powder. Note: Compound S5 is a 1:1 mixture of atropisomers

${ }^{1} \mathbf{H}$ NMR $\left(500 \mathrm{MHz}, \mathrm{CD}_{2} \mathrm{Cl}_{2}\right) \delta 8.03(\mathrm{tt}, J=7.2,3.4 \mathrm{~Hz}, 1 \mathrm{H}), 7.41$ $(\mathrm{ddd}, J=5.8,3.5,1.4 \mathrm{~Hz}, 2 \mathrm{H}), 7.28(\mathrm{~d}, J=8.2 \mathrm{~Hz}, 1 \mathrm{H}), 7.10(\mathrm{dd}, J=10.0,1.8 \mathrm{~Hz}, 2 \mathrm{H}), 6.98-$ $6.91(\mathrm{~m}, 2 \mathrm{H}), 6.75$ (dt, $J=6.1,3.5 \mathrm{~Hz}, 1 \mathrm{H}), 3.10$ (hept, $J=7.0 \mathrm{~Hz}, 1 \mathrm{H}), 2.67-2.59(\mathrm{~m}, 4 \mathrm{H}), 2.55$ $(\mathrm{td}, J=14.6,13.4,7.8 \mathrm{~Hz}, 1 \mathrm{H}), 1.55(\mathrm{dd}, J=9.4,6.7 \mathrm{~Hz}, 6 \mathrm{H}), 1.48(\mathrm{~d}, J=14.0 \mathrm{~Hz}, 9 \mathrm{H}), 1.40$ $(\mathrm{dd}, J=7.0,4.0 \mathrm{~Hz}, 6 \mathrm{H}), 1.35$ (d, $J=13.9 \mathrm{~Hz}, 9 \mathrm{H}), 0.94$ (d, $J=6.6 \mathrm{~Hz}, 3 \mathrm{H}), 0.82$ (d, $J=6.7 \mathrm{~Hz}$, $3 \mathrm{H})$.

${ }^{13}$ C NMR $\left(126 \mathrm{MHz}, \mathrm{CD}_{2} \mathrm{Cl}_{2}\right) \delta 158.4,153.5,153.2,147.3,147.2,145.6,145.5,140.0,140.0$, $140.0,140.0,138.8,138.8,136.4,136.2,135.8,135.4,135.3,130.5,130.5,126.5,126.2,126.1$, $125.9,125.6,125.6,125.5,125.5,125.4,124.8,124.3,124.1,124.1,124.0,124.0,119.4,119.4$, $119.4,119.3,39.8,39.6,39.3,39.2,34.9,32.2,32.1,32.1,31.5,31.0,31.0,29.4,26.0,25.8,25.0$, 24.9, 24.7, 23.5.

${ }^{19}$ F NMR $\left(471 \mathrm{MHz}, \mathrm{CD}_{2} \mathrm{Cl}_{2}\right) \delta-62.89$.

${ }^{31} \mathbf{P}$ NMR $\left(203 \mathrm{MHz}, \mathrm{CD}_{2} \mathrm{Cl}_{2}\right) \delta 52.43$.

IR (neat): 3062, 2965, 2927, 2866, 1604, 1585, 1459, 1424, 1393, 1383, 1360, 1322, 1282, 1253 , 1187, 1154, 1128, 1104, 1073, 1012, 990, 936, 872, 813, 773, 758, 749, 739, 713, 645, $597 \mathrm{~cm}^{-1}$ M.P.: $158-160{ }^{\circ} \mathrm{C}($ dec.)

EA Calcd. for $\mathrm{C}_{37} \mathrm{H}_{51} \mathrm{BrF}_{3} \mathrm{PPd}$ : C, 57.71; H, 6.68. Found: C, 57.67; H, 6.52.

\section{(t-BuXPhos)Pd[(4-OMe)Ph]Cl (S6)}
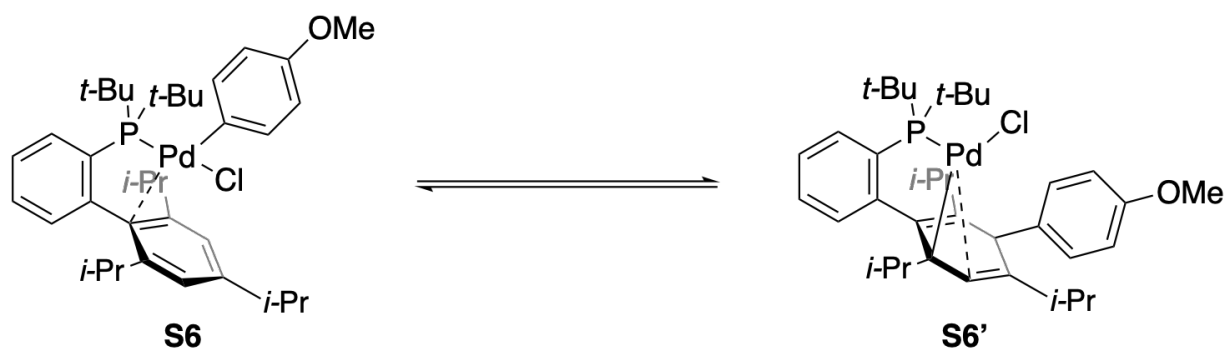

Compound S6 was prepared according to the General Procedure A using (cod) $\mathrm{Pd}\left(\mathrm{CH}_{2} \mathrm{TMS}\right)_{2}$ (389 mg, $1.00 \mathrm{mmol}, 1$ equiv), $t$-BuXPhos (425 mg, $1.00 \mathrm{mmol}, 1.00$ equiv), 4-chloroanisole 
(184 $\mu \mathrm{L}, 1.50 \mathrm{mmol}, 1.5$ equiv), and pentane (degassed, $10.0 \mathrm{~mL}$ ). The precipitate was collected on a fritted funnel to yield S6 (551 $\mathrm{mg}, 82 \%)$ as a pale-yellow powder.

${ }^{1} \mathbf{H}$ NMR $\left(500 \mathrm{MHz}, \mathrm{CD}_{2} \mathrm{Cl}_{2}\right) \delta 8.03-7.96(\mathrm{~m}, 1 \mathrm{H}), 7.40(\mathrm{dd}, J=5.9,3.5 \mathrm{~Hz}, 2 \mathrm{H}), 7.08(\mathrm{~s}, 2 \mathrm{H})$, $6.93(\mathrm{~d}, J=7.9 \mathrm{~Hz}, 2 \mathrm{H}), 6.79(\mathrm{dt}, J=6.1,3.4 \mathrm{~Hz}, 1 \mathrm{H}), 6.52(\mathrm{~d}, J=8.5 \mathrm{~Hz}, 2 \mathrm{H}), 3.67(\mathrm{~s}, 3 \mathrm{H})$, 3.01 (hept, $J=7.0 \mathrm{~Hz}, 1 \mathrm{H}), 2.54$ (hept, $J=6.7 \mathrm{~Hz}, 2 \mathrm{H}), 1.58$ (d, $J=6.8 \mathrm{~Hz}, 6 \mathrm{H}), 1.41$ (s, 9H), $1.38(\mathrm{~s}, 9 \mathrm{H}), 1.36(\mathrm{~d}, J=7.0 \mathrm{~Hz}, 6 \mathrm{H}), 0.90(\mathrm{~d}, J=6.7 \mathrm{~Hz}, 6 \mathrm{H})$.

${ }^{13}$ C NMR $\left(126 \mathrm{MHz}, \mathrm{CD}_{2} \mathrm{Cl}_{2}\right) \delta 177.5,177.4,159.5,157.3,157.3,156.8,152.2,147.9,147.8$, $146.9,138.2,138.2,136.9,136.7,135.8,135.1,134.8,134.7,134.4,132.3,132.2,131.4,131.3$, $130.4,130.4,129.9,129.5,129.2,127.1,127.1,126.1,126.0,125.6,125.5,124.8,122.6,122.6$, $120.8,113.9,112.7,97.9,97.9,65.7,65.6,55.6,55.3,55.3,55.3,39.6,39.6,39.6,39.5,39.5$, $38.7,38.5,37.8,37.7,35.0,35.0,35.0,34.2,34.2,32.5,32.3,32.2,31.8,31.8,31.7,29.6,29.6$, 25.6, 24.8, 24.7, 23.0, 23.0, 22.1, 22.1, 21.2, 21.1, 20.9.

${ }^{31}$ P NMR $\left(203 \mathrm{MHz}, \mathrm{CD}_{2} \mathrm{Cl}_{2}\right) \delta 67.05,52.38$.

IR (neat): 3053, 2958, 2925, 2897, 2859, 2835, 1601, 1580, 1568, 1552, 1478, 1459, 1440, 1419 , $1400,1388,1374,1367,1357,1331,1312,1293,1270,1253,1234,1180,1170,1159,1125$, 1097, 1073, 1057, 1050, 1031, 1021, 1000, 936, 874, 839, 806, 794, 780, 758, 732, 697, 666, $645,585 \mathrm{~cm}^{-1}$

M.P.: $161-162^{\circ} \mathrm{C}$ (dec.)

HRMS (ESI) Calcd. For $\mathrm{C}_{36} \mathrm{H}_{52} \mathrm{OPPd}(\mathrm{M}-\mathrm{Cl})^{+}$: 637.2785. Found: 637.2803.

\section{(t-BuBrettPhos)Pd[(4-CF $) \mathrm{Ph}] \mathrm{Cl}(\mathrm{S} 7)$}
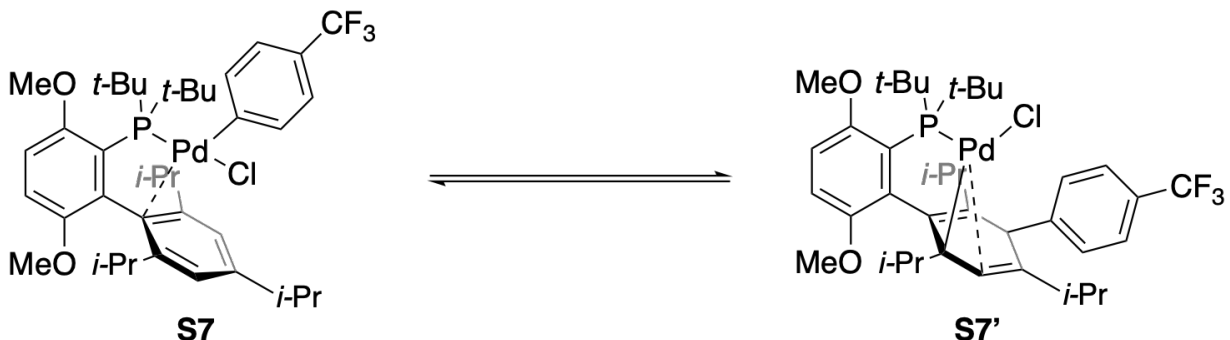

Compound S7 was prepared according to General Procedure A using (cod)Pd( $\left.\mathrm{CH}_{2} \mathrm{TMS}\right)_{2}(194$ mg, 0.500 mmol, 1 equiv), $t$-BuBrettPhos (243 mg, 0.500 mmol, 1.0 equiv), 4chlorobenzotrifluoride ( $100 \mu \mathrm{L}, 0.75 \mathrm{mmol}, 1.5$ equiv), and pentane (degassed, $5.0 \mathrm{~mL}$ ). The precipitate was collected on a fritted funnel to yield S7 $(291 \mathrm{mg}, 75 \%)$ as a tan powder.

${ }^{1}$ H NMR $\left(500 \mathrm{MHz}, \mathrm{CD}_{2} \mathrm{Cl}_{2}\right) \delta 7.27(\mathrm{~d}, J=7.7 \mathrm{~Hz}, 2 \mathrm{H}), 7.04(\mathrm{~s}, 2 \mathrm{H}), 7.02(\mathrm{~d}, J=8.3 \mathrm{~Hz}, 2 \mathrm{H})$, $6.94(\mathrm{dd}, J=8.9,2.7 \mathrm{~Hz}, 1 \mathrm{H}), 6.89(\mathrm{~d}, J=8.3 \mathrm{~Hz}, 1 \mathrm{H}), 3.80(\mathrm{~s}, 3 \mathrm{H}), 3.34(\mathrm{~s}, 3 \mathrm{H}), 2.98$ (hept, $J=$ $6.9 \mathrm{~Hz}, 1 \mathrm{H}), 2.60$ (hept, $J=6.7 \mathrm{~Hz}, 2 \mathrm{H}), 1.60$ (d, $J=6.8 \mathrm{~Hz}, 6 \mathrm{H}), 1.41$ (s, 9H), 1.38 (s, 9H), 1.34 $(\mathrm{d}, J=6.9 \mathrm{~Hz}, 6 \mathrm{H}), 0.84(\mathrm{~d}, J=6.7 \mathrm{~Hz}, 6 \mathrm{H})$.

${ }^{13} \mathrm{C}$ NMR $\left(126 \mathrm{MHz}, \mathrm{CD}_{2} \mathrm{Cl}_{2}\right) \delta 158.7,155.0,154.3,154.3,152.3,152.2,147.4,147.4,147.3$, $139.7,139.6,139.6,138.6,138.4,132.3,132.1,128.0,127.8,126.6,125.9,125.9,124.9,124.6$, $124.4,124.3,124.1,121.6,121.5,121.5,121.5,116.2,116.1,114.2,113.9,113.9,110.9,110.9$, $110.7,110.7,99.2,99.2,55.1,54.8,54.5,54.2,41.3,41.2,41.2,35.1,33.9,33.0,32.7,32.7,31.7$, $30.4,30.3,25.9,24.8,24.7,22.7,22.7,22.5,22.5,22.4,21.4,21.4,20.4,20.3,20.3$.

${ }^{19}$ F NMR $\left(471 \mathrm{MHz}, \mathrm{CD}_{2} \mathrm{Cl}_{2}\right) \delta-62.91,-63.44$.

${ }^{31} \mathbf{P}$ NMR $\left(203 \mathrm{MHz}, \mathrm{CD}_{2} \mathrm{Cl}_{2}\right) \delta 80.78,73.97$. 
IR (neat): 3077, 3017, 2977, 2956, 2925, 2897, 2863, 2828, 1604, 1585, 1481, 1455, 1419, 1381, $1360,1322,1265,1237,1185,1170,1156,1109,1097,1083,1066,1052,1007,941,886,874$, $841,832,813,784,768,756,723,678,649,619 \mathrm{~cm}^{-1}$

M.P.: $173-176^{\circ} \mathrm{C}(\mathrm{dec}$.)

EA Calcd. for $\mathrm{C}_{38} \mathrm{H}_{53} \mathrm{ClF}_{3} \mathrm{O}_{2} \mathrm{PPd}$ : C, 59.15; H, 6.92. Found: C, 59.12; H, 6.93.

(t-BuBrettPhos)Pd[(4-CF 3$)$ Ph]Br (S8)
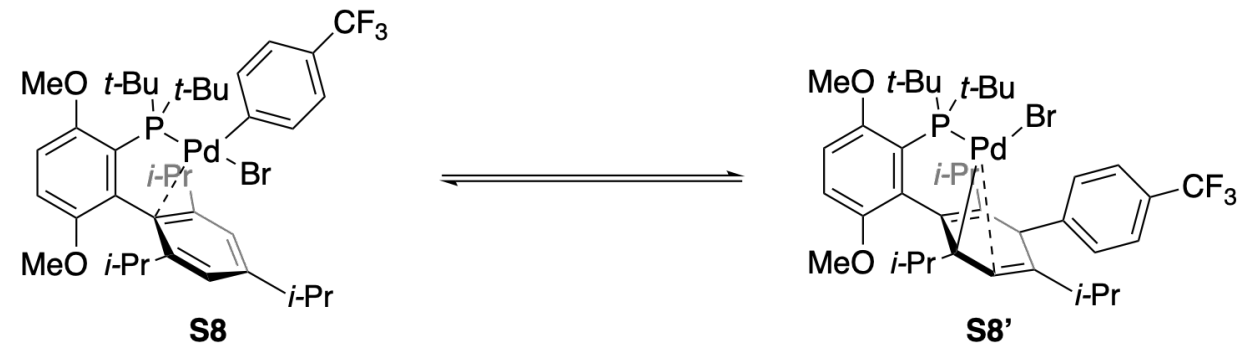

Compound S8 was prepared according to General Procedure A using (cod) $\mathrm{Pd}\left(\mathrm{CH}_{2} \mathrm{TMS}\right)_{2}(194$ $\mathrm{mg}, \quad 0.500$ mmol, 1 equiv), $t$-BuBrettPhos (243 $\mathrm{mg}, 0.500 \mathrm{mmol}, 1.0$ equiv), 4bromobenzotrifluoride (105 $\mu \mathrm{L}, 0.75 \mathrm{mmol}, 1.5$ equiv), and pentane (degassed, $5.0 \mathrm{~mL}$ ). The precipitate was collected on a fritted funnel to yield S8 (301 $\mathrm{mg}, 74 \%)$ as a pale-yellow powder.

${ }^{1}$ H NMR $\left(500 \mathrm{MHz}, \mathrm{CD}_{2} \mathrm{Cl}_{2}\right) \delta 7.29(\mathrm{~d}, J=8.1 \mathrm{~Hz}, 2 \mathrm{H}), 7.07(\mathrm{~s}, 2 \mathrm{H}), 7.02(\mathrm{~d}, J=8.2 \mathrm{~Hz}, 2 \mathrm{H})$, $6.95(\mathrm{dd}, J=8.9,2.6 \mathrm{~Hz}, 1 \mathrm{H}), 6.90-6.87(\mathrm{~m}, 1 \mathrm{H}), 3.80$ (s, 3H), 3.34 (s, 3H), 3.05 (hept, $J=6.9$ Hz, 1H), 2.61 (hept, $J=6.8 \mathrm{~Hz}, 2 \mathrm{H}), 1.60(\mathrm{~d}, J=6.8 \mathrm{~Hz}, 3 \mathrm{H}), 1.41(\mathrm{~s}, 9 \mathrm{H}), 1.38(\mathrm{~s}, 9 \mathrm{H}), 1.37$ (d, $J=6.9 \mathrm{~Hz}, 6 \mathrm{H}), 0.84(\mathrm{~d}, J=6.6 \mathrm{~Hz}, 6 \mathrm{H})$.

${ }^{13}$ C NMR $\left(126 \mathrm{MHz}, \mathrm{CD}_{2} \mathrm{Cl}_{2}\right) \delta 172.6,172.5,158.5,155.3,155.3,155.0,154.4,154.3,152.2$, $152.1,151.7,151.7,143.7,143.6,143.0,143.0,143.0,143.0,140.6,140.5,138.3,138.1,136.5$, $136.3,135.0,134.9,132.7,132.1,130.0,129.7,129.5,128.7,127.7,127.6,126.5,125.9,125.7$, $125.7,125.6,125.2,125.1,125.0,124.8,124.5,124.4,124.2,124.0,123.8,122.2,121.2,121.2$, $121.2,121.1,119.1,118.9,116.4,116.4,114.3,114.3,113.9,113.9,111.0,111.0,110.8,110.8$, 99.8, 99.8, 68.3, 68.2, 55.2, 54.7, 54.6, 54.2, 52.3, 52.2, 41.6, 41.5, 40.2, 40.1, 39.7, 39.6, 34.9, $34.2,34.1,33.0,33.0,32.7,32.7,31.7,30.6,30.5,26.0,25.9,25.9,25.9,24.9,24.9,24.7,24.6$, 24.6, 24.6, 24.6, 22.6, 22.5, 22.4, 22.4, 22.4, 21.5, 21.4, 20.4, 20.3, 20.2.

${ }^{19}$ F NMR $\left(471 \mathrm{MHz}, \mathrm{CD}_{2} \mathrm{Cl}_{2}\right) \delta-62.86$.

${ }^{31} \mathbf{P}$ NMR $\left(203 \mathrm{MHz}, \mathrm{CD}_{2} \mathrm{Cl}_{2}\right) \delta 82.43,71.73$.

IR (neat): 3077, 3017, 2977, 2953, 2925, 1897, 1866, 1828, 1606, 1580, 1478, 1455, 1417, 1381, $1367,1360,1320,1265,1239,1185,1170,1156,1111,1097,1083,1066,1054,1007,941,889$, $872,829,810,787,768,756,723,678,647,619 \mathrm{~cm}^{-1}$

M.P.: $181-182^{\circ} \mathrm{C}($ dec. $)$

EA Calcd. for $\mathrm{C}_{38} \mathrm{H}_{53} \mathrm{BrF}_{3} \mathrm{O}_{2} \mathrm{PPd}$ : C, 55.92; H, 6.55. Found: C, 55.96; H, 6.71. 


\section{(t-BuBrettPhos)Pd[(4-CF 3$)$ Ph]OTf (S9)}

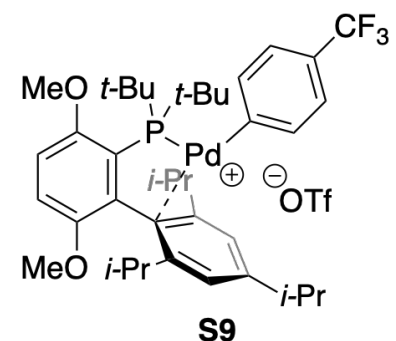

S9

Compound S9 was prepared according to General Procedure A using (cod) $\mathrm{Pd}\left(\mathrm{CH}_{2} \mathrm{TMS}\right)_{2}$ (194 mg, $0.500 \mathrm{mmol}, 1$ equiv), $t$-BuBrettPhos (243 mg, 0.500 mmol, 1.0 equiv), 4-(trifluoromethyl)phenyl trifluoromethanesulfonate $(220 \mathrm{mg}, 0.75 \mathrm{mmol}, 1.5 \mathrm{equiv})$, and pentane (degassed, $5.0 \mathrm{~mL}$ ). The precipitate was collected on a fritted funnel to yield $\mathbf{S 9}$ (364 $\mathrm{mg}, 82 \%$ ) as a yellow-orange powder.

${ }^{1}$ H NMR $\left(600 \mathrm{MHz}, \mathrm{CD}_{2} \mathrm{Cl}_{2}\right) \delta 7.38(\mathrm{dd}, J=8.1,3.4 \mathrm{~Hz}, 2 \mathrm{H}), 7.34$ $(\mathrm{d}, J=9.1 \mathrm{~Hz}, 1 \mathrm{H}), 7.25-7.20(\mathrm{~d}, J=8.4 \mathrm{~Hz}, 5 \mathrm{H}), 3.87(\mathrm{~s}, 3 \mathrm{H})$, $3.80(\mathrm{~s}, 3 \mathrm{H}), 2.60$ (hept, $J=6.7 \mathrm{~Hz}, 2 \mathrm{H}), 2.33$ (heptd, $J=7.0,1.7 \mathrm{~Hz}, 1 \mathrm{H}), 1.52$ (d, $J=6.7 \mathrm{~Hz}$, $6 \mathrm{H}), 1.36(\mathrm{~s}, 9 \mathrm{H}), 1.33(\mathrm{~s}, 9 \mathrm{H}), 1.18(\mathrm{~d}, J=6.7 \mathrm{~Hz}, 6 \mathrm{H}), 0.88(\mathrm{~d}, J=7.0 \mathrm{~Hz}, 6 \mathrm{H})$.

${ }^{13}$ C NMR $\left(151 \mathrm{MHz}, \mathrm{CD}_{2} \mathrm{Cl}_{2}\right) \delta 154.6,152.9,152.8,151.2,146.6,146.5,145.4,145.3,140.2$, $140.2,140.1,131.4,131.3,129.4,129.2,128.7,128.5,128.3,128.1,127.4,125.6,124.3,124.2$, $124.2,124.2,124.2,123.8,122.5,120.4,117.4,117.4,117.0,117.0,115.2,114.5,114.4,55.4$, $55.2,42.3,42.2,33.8,31.8,31.7,25.5,23.4,22.0$.

${ }^{19}$ F NMR $\left(565 \mathrm{MHz}, \mathrm{CD}_{2} \mathrm{Cl}_{2}\right) \delta-63.70,-79.86$.

${ }^{31} \mathbf{P}$ NMR $\left(203 \mathrm{MHz}, \mathrm{CD}_{2} \mathrm{Cl}_{2}\right) \delta 113.60$.

IR (neat): 3048, 2970, 2927, 2871, 1580, 1471, 1426, 1393, 1367, 1320, 1260, 1220, 1187, 1154, $1114,1099,1069,1054,1028,1002,941,886,818,796,770,749,723,711,633,574 \mathrm{~cm}^{-1}$ M.P.: $212-215^{\circ} \mathrm{C}($ dec.)

EA Calcd. for $\mathrm{C}_{39} \mathrm{H}_{53} \mathrm{~F}_{6} \mathrm{O}_{5} \mathrm{PPdS}$ : C, 52.91; H, 6.03. Found: C, 52.65; H, 6.04.

(t-BuXPhos)Pd(X14)I (S10)

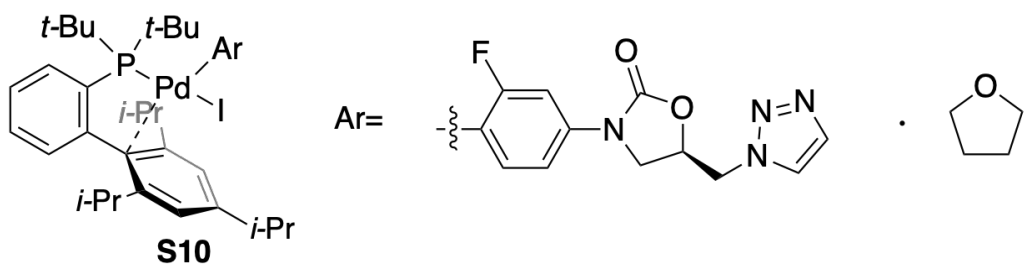

Compound S10 was prepared according to General Procedure B using $[(t-\mathrm{BuXPhos}) \mathrm{Pd}]_{2}(\operatorname{cod})$ (293 mg, $0.250 \mathrm{mmol}, 1$ equiv), X14 (194 mg, $0.5 \mathrm{mmol}, 1.0$ equiv w.r.t. [Pd]), and THF (5.0 $\mathrm{mL}$ ). The precipitate was collected on a fritted funnel to yield $\mathbf{S 1 0}(376 \mathrm{mg}, 82 \%)$ as an orange powder.

${ }^{1} \mathbf{H}$ NMR $\left(600 \mathrm{MHz}, \mathrm{CD}_{2} \mathrm{Cl}_{2}\right) \delta 8.00(\mathrm{q}, J=5.9 \mathrm{~Hz}, 1 \mathrm{H}), 7.78(\mathrm{~s}, 1 \mathrm{H}), 7.68(\mathrm{~s}, 1 \mathrm{H}), 7.52$ (br s, 1H), $7.42-7.32(\mathrm{~m}, 2 \mathrm{H}), 7.14-7.09(\mathrm{~m}, 2 \mathrm{H}), 7.06-7.01(\mathrm{~m}, 1 \mathrm{H}), 6.87-6.79(\mathrm{~m}, 1 \mathrm{H}), 6.64$ $(\mathrm{dt}, J=5.3,3.2 \mathrm{~Hz}, 1 \mathrm{H}), 4.98(\mathrm{dd}, J=6.5,3.4 \mathrm{~Hz}, 1 \mathrm{H}), 4.76(\mathrm{dd}, J=14.7,4.0 \mathrm{~Hz}, 1 \mathrm{H}), 4.68$ (ddd, $J=14.7,6.1,3.2 \mathrm{~Hz}, 1 \mathrm{H}), 4.08(\mathrm{t}, J=9.1 \mathrm{~Hz}, 1 \mathrm{H}), 3.75(\mathrm{dd}, J=9.4,6.5 \mathrm{~Hz}, 1 \mathrm{H}), 3.17$ (hept, $J=7.0 \mathrm{~Hz}, 1 \mathrm{H}), 2.66-2.43(\mathrm{~m}, 2 \mathrm{H}), 1.61(\mathrm{~d}, J=6.9 \mathrm{~Hz}, 3 \mathrm{H}), 1.53-1.44(\mathrm{~m}, 9 \mathrm{H}), 1.40$ $(\mathrm{dd}, J=7.0,3.1 \mathrm{~Hz}, 6 \mathrm{H}), 1.35(\mathrm{~d}, J=14.0 \mathrm{~Hz}, 8 \mathrm{H}), 1.26-1.19(\mathrm{~m}, 1 \mathrm{H}), 1.19-1.13(\mathrm{~m}, 1 \mathrm{H})$, $0.94(\mathrm{~d}, J=6.7 \mathrm{~Hz}, 2 \mathrm{H}), 0.89(\mathrm{~d}, J=6.6 \mathrm{~Hz}, 3 \mathrm{H}), 0.82(\mathrm{~d}, J=6.6 \mathrm{~Hz}, 3 \mathrm{H})$.

${ }^{13}$ C NMR $\left(151 \mathrm{MHz}, \mathrm{CD}_{2} \mathrm{Cl}_{2}\right) \delta 165.0,163.5,159.0,154.3,154.0,153.6,146.9,146.7,142.2$, $142.1,136.5,136.3,136.0,136.0,135.7,135.2$, 135.2, 134.4, 130.6, 128.1, 126.2, 126.0, 126.0, $125.9,125.4,123.8,120.8,120.1,111.9,104.3,104.1,98.1,97.9,71.0,71.0,68.1,52.6,52.5$, 
47.7, 47.7, 40.1, 40.0, 39.9, 39.8, 34.5, 34.5, 33.2, 32.1, 32.0, 32.0, 31.9, 31.3, 31.2, 31.1, 31.1, $31.0,30.9,28.1,26.5,26.0,25.6,25.5,25.0,24.9,24.8,24.2,23.7,22.8$.

${ }^{19}$ F NMR $\left(565 \mathrm{MHz}, \mathrm{CD}_{2} \mathrm{Cl}_{2}\right) \delta-80.78$.

${ }^{31} \mathbf{P}$ NMR $\left(202 \mathrm{MHz}, \mathrm{CD}_{2} \mathrm{Cl}_{2}\right) \delta 47.27$.

IR (neat): 2965, 2930, 2901, 2866, 1758, 1599, 1563, 1473, 1414, 1383, 1360, 1315, 1282, 1220, 1182, 1128, 1109, 1071, 1021, 938, 903, 872, 853, 836, 777, 747, 735, 694, $671 \mathrm{~cm}^{-1}$

M.P.: $186-189^{\circ} \mathrm{C}($ dec.)

HRMS (ESI) Calcd. For $\mathrm{C}_{41} \mathrm{H}_{55} \mathrm{FN}_{4} \mathrm{O}_{2} \mathrm{PPd}(\mathrm{M}-\mathrm{I})^{+}:$791.3076. Found: 791.3093.

(t-BuXPhos)Pd(Indometacin)Cl (S11)
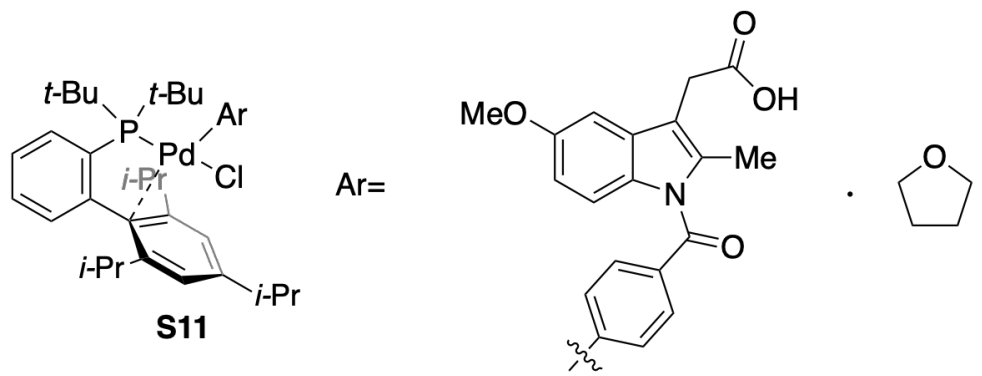

Compound S11 was prepared according to General Procedure B using $[(t \text {-BuXPhos }) \mathrm{Pd}]_{2}(\operatorname{cod})$ (293 mg, $0.250 \mathrm{mmol}, 1$ equiv), Indometacin (179 mg, $0.5 \mathrm{mmol}, 1.0$ equiv w.r.t. [Pd]), and THF $(5.0 \mathrm{~mL})$. The precipitate was collected on a fritted funnel to yield $\mathbf{S 1 1}(454 \mathrm{mg}, 96 \%)$ as a grey powder. Note: Samples of $\mathbf{S 1 1}$ contain 1 equivalent of THF and the isolated yield is based off of S11·THF

${ }^{1} \mathbf{H}$ NMR $\left(600 \mathrm{MHz}, \mathrm{CD}_{2} \mathrm{Cl}_{2}\right) \delta 8.02,8.02,8.01,8.01,8.00,8.00,7.44,7.44,7.44,7.43,7.43$, $7.43,7.43,7.42,7.35,7.35,7.33,7.33,7.16,7.15,7.10,6.92,6.91,6.82,6.81,6.80,6.80,6.79$, $6.79,6.78,6.56,3.79,3.68,3.06,3.05,3.04,3.03,3.01,3.00,2.99,2.59,2.58,2.56,2.55,2.54$, $2.53,2.52,2.32,2.32,1.59,1.58,1.46,1.44,1.37,1.36,0.92,0.91$.

${ }^{13}$ C NMR $\left(151 \mathrm{MHz}, \mathrm{CD}_{2} \mathrm{Cl}_{2}\right) \delta 175.8,170.0,158.1,156.0,153.1,148.5,147.7,147.6,139.1$, $139.1,136.5,136.3,135.7,134.8,134.7,131.4,130.7,130.7,130.5,130.2,127.1,126.3,126.3$, $125.1,124.3,124.3,115.3,111.5,111.2,100.9,56.0,40.0,39.9,35.0,31.9,31.8,31.8,30.2$, 25.6, 24.9, 24.8 .

${ }^{31} \mathbf{P}$ NMR $\left(202 \mathrm{MHz}, \mathrm{CD}_{2} \mathrm{Cl}_{2}\right) \delta 54.27$.

IR (neat): 3058, 2963, 2925, 2868, 2560, 1720, 1677, 1616, 1606, 1563, 1476, 1459, 1436, 1421, 1383, 1360, 1308, 1286, 1263, 1225, 1170, 1142, 1130, 1099, 1064, 1043, 1019, 1007, 962, 943, $919,896,872,855,834,825,808,796,773,758,749,732,713,692,654,647,600 \mathrm{~cm}^{-1}$

M.P.: $165-167^{\circ} \mathrm{C}(\mathrm{dec}$.

HRMS (ESI) Calcd. For $\mathrm{C}_{48} \mathrm{H}_{61} \mathrm{NO}_{4} \mathrm{PPd}(\mathrm{M}-\mathrm{Cl})^{+}:$852.3368. Found: 852.3381. 


\section{(t-BuBrettPhos)Pd(rivaroxaban)Cl (S12)}
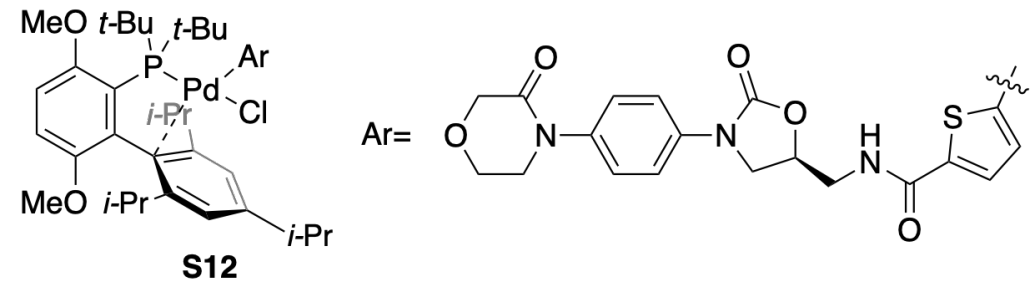

Compound S12 was prepared according to General Procedure B using [( $t$-BuBrettPhos $) \mathrm{Pd}]_{2}(\mathrm{cod})$ (323 mg, $0.250 \mathrm{mmol}, 1$ equiv), rivaroxaban (218 mg, $0.5 \mathrm{mmol}, 1.0$ equiv w.r.t. [Pd]), and THF $(5.0 \mathrm{~mL})$. The precipitate was collected on a fritted funnel to yield $\mathbf{S 1 2}(483 \mathrm{mg}, 88 \%)$ as a green powder. Note: Samples of $\mathbf{S 1 2}$ contain 1 equivalent of THF and the isolated yield is based off of S12·THF

${ }^{1}$ H NMR $\left(600 \mathrm{MHz}, \mathrm{CD}_{2} \mathrm{Cl}_{2}\right) \delta 7.60-7.55(\mathrm{~m}, 1 \mathrm{H}), 7.17-7.06(\mathrm{~m}, 5 \mathrm{H}), 6.95(\mathrm{dd}, J=9.0,2.9$ $\mathrm{Hz}, 1 \mathrm{H}), 6.89(\mathrm{~d}, J=8.9 \mathrm{~Hz}, 1 \mathrm{H}), 6.83(\mathrm{br} \mathrm{s}, 1 \mathrm{H}), 6.56(\mathrm{~d}, J=3.9 \mathrm{~Hz}, 1 \mathrm{H}), 4.74(\mathrm{tt}, J=8.6,5.2$ $\mathrm{Hz}, 1 \mathrm{H}), 4.26-4.19(\mathrm{~m}, 3 \mathrm{H}), 3.96(\mathrm{t}, J=5.1 \mathrm{~Hz}, 2 \mathrm{H}), 3.94-3.87(\mathrm{~m}, 1 \mathrm{H}), 3.80(\mathrm{~s}, 3 \mathrm{H}), 3.71$ $(\mathrm{dd}, J=9.7,5.6 \mathrm{~Hz}, 1 \mathrm{H}), 3.56(\mathrm{tq}, J=17.4,7.1,6.0 \mathrm{~Hz}, 2 \mathrm{H}), 3.34(\mathrm{~s}, 3 \mathrm{H}), 3.25(\mathrm{~d}, J=13.6 \mathrm{~Hz}$, $1 \mathrm{H}), 3.01$ (hept, $J=7.0 \mathrm{~Hz}, 1 \mathrm{H}), 2.80-2.73(\mathrm{~m}, 1 \mathrm{H}), 2.47-2.39(\mathrm{~m}, 1 \mathrm{H}), 1.59$ (dd, $J=8.5,6.8$ $\mathrm{Hz}, 6 \mathrm{H}), 1.54(\mathrm{~d}, J=15.3 \mathrm{~Hz}, 9 \mathrm{H}), 1.42-1.36(\mathrm{~m}, 6 \mathrm{H}), 1.32(\mathrm{~d}, J=15.5 \mathrm{~Hz}, 9 \mathrm{H}), 0.89(\mathrm{~d}, J=$ $6.6 \mathrm{~Hz}, 3 \mathrm{H}), 0.77(\mathrm{~d}, J=6.6 \mathrm{~Hz}, 3 \mathrm{H})$.

${ }^{13} \mathrm{C}$ NMR $\left(151 \mathrm{MHz}, \mathrm{CD}_{2} \mathrm{Cl}_{2}\right) \delta 167.0,166.7,162.4,161.9,160.1,158.1,155.6,155.0,154.7$, $154.1,154.1,152.2,152.1,140.5,138.3,138.2,137.5,137.2,136.7,136.2,129.2,128.3,127.8$, 127.7, 127.6, 126.5, 126.1, 125.7, 125.0, 119.2, 118.8, 114.3, 114.2, 114.1, 111.3, 111.3, 72.0, $71.6,68.9,64.5,54.7,54.3,49.9,49.1,42.2,42.1,41.7,41.5,41.4,35.2,33.0,33.0,32.2,32.0$, 32.0, 31.4, 25.9, 25.6, 24.9, 24.8, 24.8, 24.5.

${ }^{31} \mathbf{P}$ NMR $\left(202 \mathrm{MHz}, \mathrm{CD}_{2} \mathrm{Cl}_{2}\right) \delta 79.61$.

IR (neat): 3301, 2960, 2927, 2904, 2863, 2830, 1746, 1665, 1637, 1606, 1582, 1535, 1516, 1473, $1457,1443,1431,1419,1407,1388,1360,1343,1322,1310,1301,1279,1263,1237,1225$, 1208, 1189, 1159, 1128, 1111, 1085, 1057, 1043, 1031, 1009, 998, 964, 941, 919, 889, 832, 808, $787,773,763,754,744,718,704,685,657,623,607 \mathrm{~cm}^{-1}$

M.P.: $197-201{ }^{\circ} \mathrm{C}($ dec.)

HRMS (ESI) Calcd. For $\mathrm{C}_{50} \mathrm{H}_{67} \mathrm{~N}_{3} \mathrm{O}_{7} \mathrm{PPdS}(\mathrm{M}-\mathrm{Cl})^{+}$: 990.3467 . Found: 990.3485 . 


\section{Pd OAC Ligand Exchange Reactions}

\section{General Procedure C: Ligand Exchange on Pharmaceutical-Derived OACs}

An oven-dried $24 \mathrm{~mL}$ reaction tube was equipped, while still hot, with a magnetic stir bar and septum cap. The tube was allowed to cool to room temperature under vacuum by piercing the septum with a needle connected to a dual manifold by a rubber hose. At this point, the cap was removed and the tube was charged with $\mathrm{OAC}(0.250 \mathrm{mmol}, 1.0$ equiv $)$ and ligand $(0.275 \mathrm{mmol}$, 1.1 equiv). The tube was recapped, the septum was pierced with a needle connected to the dual manifold by a rubber hose, and the tube was evacuated and backfilled with nitrogen (the evacuation/backfill process was repeated a total of three times). Under nitrogen, THF $(2.5 \mathrm{~mL})$ was added via syringe. The tube was then placed in a preheated oil bath (bath temperature $=60$ ${ }^{\circ} \mathrm{C}$ ). The reaction mixture was allowed to stir at $60{ }^{\circ} \mathrm{C}$ for $1 \mathrm{~h}$. At this point, the reaction vessel was removed from the oil bath and the reaction mixture was allowed to cool to room temperature. The reaction tube was then opened to air and the reaction mixture was diluted with THF $(2.5 \mathrm{~mL})$ and filtered through a $\sim 1 \mathrm{~cm}$ plug of Celite ${ }^{\circledR}$ into a $50 \mathrm{~mL}$ round-bottom flask. The Celite ${ }^{\circledR}$ plug was further washed with THF $(15 \mathrm{~mL})$. The combined filtrate solution was concentrated in vacuo with the assistance of a rotary evaporator. The resulting residue was dissolved in THF $(2.5 \mathrm{~mL})$ and pipetted into a $50 \mathrm{~mL}$ beaker containing $25 \mathrm{~mL}$ of pentane dropwise to precipitate the complex. The precipitate was collected on a fritted funnel and washed with pentane $(25 \mathrm{~mL})$ and dried in vacuo on a Schlenk line overnight $(\sim 16 \mathrm{~h})$ to afford the desired complex.

\section{General Procedure D: Ligand Exchange on OACs of Simple Aryl Halides}

An oven-dried $24 \mathrm{~mL}$ reaction tube was equipped, while still hot, with a magnetic stir bar and septum cap. The tube was allowed to cool to room temperature under vacuum by piercing the septum with a needle connected to a dual manifold by a rubber hose. At this point, the cap was removed and the tube was charged with $\mathrm{OAC}(0.250 \mathrm{mmol}, 1.0$ equiv $)$ and ligand $(0.275 \mathrm{mmol}$, 1.1 equiv). The tube was recapped, the septum was pierced with a needle connected to the dual manifold by a rubber hose, and the tube was evacuated and backfilled with nitrogen (the evacuation/backfill process was repeated a total of three times). Under nitrogen, $n$-hexane (2.5 $\mathrm{mL}$ ) was added via syringe. The tube was then placed in a preheated oil bath (bath temperature $=$ $60{ }^{\circ} \mathrm{C}$ ). The reaction mixture was allowed to stir at $60{ }^{\circ} \mathrm{C}$ for $1 \mathrm{~h}$. At this point, the reaction vessel was removed from the oil bath and the mixture was allowed to cool to room temperature. The reaction tube was then opened to air and the reaction mixture was diluted with pentane (2.5 $\mathrm{mL}$ ) to assist with precipitation. The precipitate was collected on a fritted funnel, washed with pentane $(25 \mathrm{~mL})$, and dried in vacuo on a Schlenk line overnight $(\sim 16 \mathrm{~h})$ to afford the desired complex. 


\section{(XPhos)Pd(rivaroxaban)Cl (2a)}

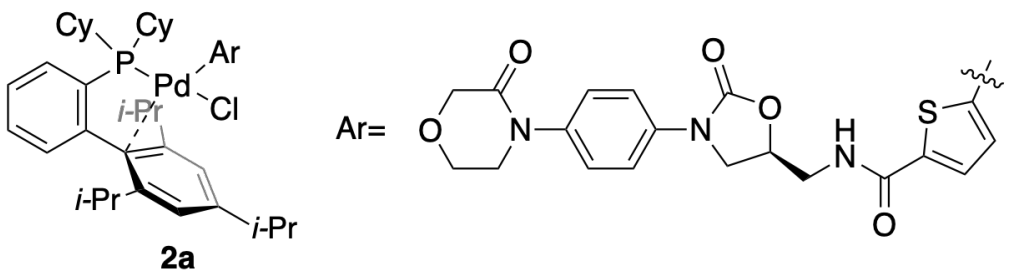

Compound 2a was prepared according to General Procedure $\mathbf{C}$ using ( $t$ BuXPhos)Pd(rivaroxaban)Cl (242 mg, $0.250 \mathrm{mmol}, 1.0$ equiv), XPhos (131 mg, $0.275 \mathrm{mmol}, 1.1$ equiv), and THF (2.5 mL). The precipitate was collected on a fritted funnel to yield $2 \mathrm{a}(202 \mathrm{mg}$, $79 \%)$ as an off-white powder.

${ }^{1} \mathbf{H}$ NMR $\left(600 \mathrm{MHz}, \mathrm{CD}_{2} \mathrm{Cl}_{2}\right)$ Complex spectrum, see below.

${ }^{13}$ C NMR $\left(151 \mathrm{MHz}, \mathrm{CD}_{2} \mathrm{Cl}_{2}\right) \delta 167.1,167.0,167.0,166.9,166.9,166.9,162.8,162.7,162.4$, $158.5,154.8,154.8,154.7,154.7,154.6,151.9,151.8,149.5,149.1,147.6,147.5,146.7,142.9$, $142.4,142.1,141.0,141.0,140.1,138.9,137.8,137.8,137.7,137.7,137.6,137.5,137.4,137.3$, 137.3, 137.2, 137.0, 136.9, 136.2, 134.8, 134.6, 133.8, 133.7, 132.5, 132.1, 132.1, 131.5, 130.9, $129.5,129.3,128.9,128.8,128.6,128.2,128.2,127.7,127.5,127.5,126.6,126.6,126.5,126.4$, $126.4,125.9,125.3,125.2,122.9,122.8,121.7,120.7,119.2,119.1,119.1,119.0,118.9,72.7$, $72.6,72.4,72.3,72.2,72.1,68.9,64.5,64.5,50.0,50.0,50.0,48.5,48.2,47.9,42.8,42.2,36.8$, $36.3,36.1,35.9,35.3,35.2,35.0,34.8,34.7,34.5,32.1,32.1,31.3,30.7,30.7,28.3,28.1,27.9$, $27.8,27.8,27.8,27.7,27.5,27.4,27.1,26.8,26.2,26.0,25.9,25.6,25.5,24.9,24.8,24.4,24.3$, $24.3,24.2,24.2,21.8$.

${ }^{31} \mathbf{P}$ NMR $\left(203 \mathrm{MHz}, \mathrm{CD}_{2} \mathrm{Cl}_{2}\right) \delta 36.28$.

IR (neat): 3344, 3058, 2958, 2927, 2856, 1758, 1656, 1606, 1516, 1462, 1440, 1417, 1400, 1381, $1360,1341,1305,1286,1270,1237,1220,1161,1125,1066,1054,995,936,919,877,851$, $832,806,780,763,742,709,687 \mathrm{~cm}^{-1}$

M.P.: $193-197{ }^{\circ} \mathrm{C}(\mathrm{dec}$.

EA Calcd. for $\mathrm{C}_{52} \mathrm{H}_{67} \mathrm{ClN}_{3} \mathrm{O}_{5}$ PPdS: C, 61.29; H, 6.63. Found: C, 61.02; H, 6.53.

\section{(RuPhos)Pd(rivaroxaban)Cl (2b)}
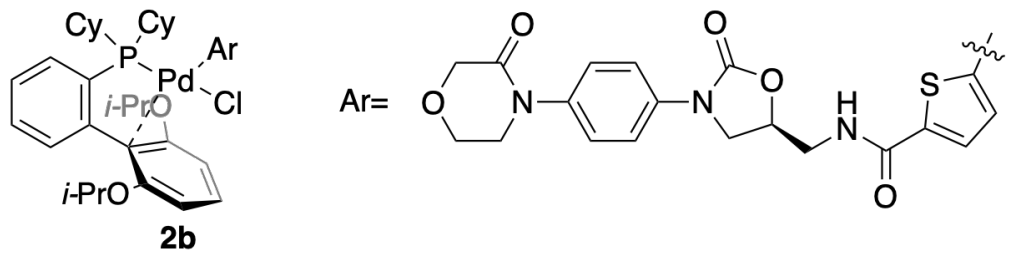

Compound 2b was prepared according to General Procedure $\mathbf{C}$ using ( $t$ BuXPhos)Pd(rivaroxaban)Cl (242 mg, $0.250 \mathrm{mmol}, 1.0$ equiv), RuPhos (128 mg, $0.275 \mathrm{mmol}$, 1.1 equiv), and THF $(2.5 \mathrm{~mL}$ ). The precipitate was collected on a fritted funnel to yield $\mathbf{2 b}$ (232 $\mathrm{mg}, 92 \%$ ) as an off-white powder. Note: samples of $\mathbf{2 b}$ contain $\sim 5 \%$ reduced rivaroxaban, however $\mathbf{2 b}$ still passes elemental analysis. 
${ }^{1} \mathbf{H}$ NMR $\left(600 \mathrm{MHz}, \mathrm{CD}_{2} \mathrm{Cl}_{2}\right) \delta 7.69(\mathrm{t}, J=8.4 \mathrm{~Hz}, 1 \mathrm{H}), 7.64-7.50(\mathrm{~m}, 3 \mathrm{H}), 7.50-7.44(\mathrm{~m}$, $1 \mathrm{H}), 7.40$ (tt, $J=7.4,1.5 \mathrm{~Hz}, 1 \mathrm{H}), 7.32-7.30(\mathrm{~m}, 1 \mathrm{H}), 7.29(\mathrm{~s}, 1 \mathrm{H}), 6.86(\mathrm{ddd}, J=7.8,3.0,1.3$ $\mathrm{Hz}, 1 \mathrm{H}), 6.63(\mathrm{dd}, J=15.2,8.5 \mathrm{~Hz}, 2 \mathrm{H}), 6.59-6.53(\mathrm{~m}, 2 \mathrm{H}), 4.81(\mathrm{dq}, J=8.6,5.8 \mathrm{~Hz}, 1 \mathrm{H})$, 4.63 (dhept, $J=12.1,6.1 \mathrm{~Hz}, 2 \mathrm{H}), 4.26(\mathrm{~s}, 2 \mathrm{H}), 4.10(\mathrm{t}, J=8.9 \mathrm{~Hz}, 1 \mathrm{H}), 3.99$ (dd, $J=5.9,4.3$ $\mathrm{Hz}, 2 \mathrm{H}), 3.86(\mathrm{dd}, J=9.2,6.6 \mathrm{~Hz}, 1 \mathrm{H}), 3.78(\mathrm{ddd}, J=14.3,6.9,5.7 \mathrm{~Hz}, 1 \mathrm{H}), 3.74-3.66(\mathrm{~m}$, 2H), 3.62 (dt, $J=14.3,5.6 \mathrm{~Hz}, 1 \mathrm{H}), 2.24$ (dddd, $J=22.5,15.3,9.7,2.7 \mathrm{~Hz}, 2 \mathrm{H}), 1.95-1.73(\mathrm{~m}$, $5 \mathrm{H}), 1.72-1.54(\mathrm{~m}, 7 \mathrm{H}), 1.39(\mathrm{dd}, J=8.1,6.0 \mathrm{~Hz}, 6 \mathrm{H}), 1.32-1.18(\mathrm{~m}, 5 \mathrm{H}), 1.18-1.06(\mathrm{~m}$, $2 \mathrm{H}), 1.02(\mathrm{dd}, J=8.0,6.0 \mathrm{~Hz}, 6 \mathrm{H}), 0.93-0.73(\mathrm{~m}, 2 \mathrm{H})$.

${ }^{13}$ C NMR $\left(151 \mathrm{MHz}, \mathrm{CD}_{2} \mathrm{Cl}_{2}\right) \delta 166.9,162.6,160.9,160.9,154.8,145.3,145.2,140.6,137.6$, 137.4, 136.9, 133.6, 133.3, 132.9, 132.8, 132.3, 132.3, 131.5, 131.5, 131.4, 129.1, 128.9, 128.8, $128.3,128.2$, 127.8, 127.7, 127.0, 126.9, 126.6, 126.4, 119.2, 119.0, 108.9, 108.8, 107.7, 107.7, $106.2,72.3,72.2,71.9,71.7,70.5,68.9,64.5,50.0,50.0,48.4,48.2,42.8,42.3,35.0,34.9,34.7$, $34.7,34.6,34.5,30.8,30.7,30.3,30.2,28.8,28.6,28.3,28.1,27.8,27.7,27.7,27.5,27.4,27.4$, 27.4, 27.1, 27.1, 27.1, 27.0, 27.0, 26.3, 26.2, 22.5, 22.3, 22.2, 21.7, 21.6.

${ }^{31} \mathbf{P}$ NMR $\left(203 \mathrm{MHz}, \mathrm{CD}_{2} \mathrm{Cl}_{2}\right) \delta 40.15$.

IR (neat): 3323, 3053, 2975, 2925, 2854, 1755, 1649, 1589, 1516, 1483, 1450, 1400, 1381, 1341, $1308,1286,1270,1244,1218,1163,1114,1054,993,938,917,896,851,832,808,739,704$, $683 \mathrm{~cm}^{-1}$

M.P.: $195-200{ }^{\circ} \mathrm{C}($ dec.)

EA Calcd. for $\mathrm{C}_{49} \mathrm{H}_{61} \mathrm{ClN}_{3} \mathrm{O}_{7} \mathrm{PPdS}$ : C, 58.33; H, 6.09. Found: C, 58.47; H, 6.19.

\section{(SPhos)Pd(rivaroxaban)Cl (2c)}

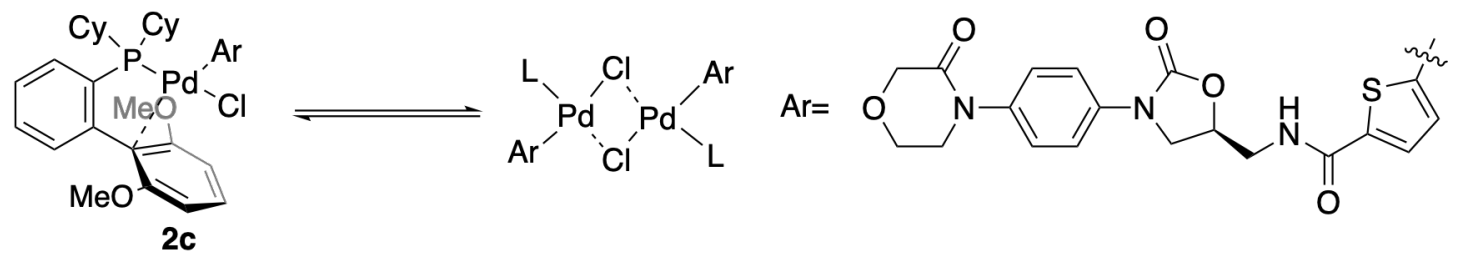

Compound 2c was prepared according to a modified General Procedure $\mathbf{C}$ using ( $t$ BuXPhos)Pd(rivaroxaban)Cl (242 mg, $0.250 \mathrm{mmol}, 1.0$ equiv), SPhos (113 mg, $0.275 \mathrm{mmol}, 1.1$ equiv), and THF $(2.5 \mathrm{~mL})$. The precipitate was collected on a fritted funnel and washed with pentane $(25 \mathrm{~mL})$ and $\mathrm{Et}_{2} \mathrm{O}(25 \mathrm{~mL})$ to yield $2 \mathrm{c}(215 \mathrm{mg}, 90 \%)$ as an off-white powder.

Note: 2c exists both as a monomeric and chloro-bridged dimeric species, as determined by ${ }^{1} \mathrm{H}$ NMR and ${ }^{31} \mathrm{P}$ NMR spectroscopy in $\mathrm{CD}_{2} \mathrm{Cl}_{2}$. At high concentrations $(\sim 30 \mathrm{mg} / \mathrm{mL})$ the dimeric species predominates. When these samples were diluted 10-fold, the monomeric species predominated. ${ }^{31} \mathrm{P}$ NMR spectra of both concentrated and dilute samples are provided.

${ }^{1}$ H NMR $\left(600 \mathrm{MHz}, \mathrm{CD}_{2} \mathrm{Cl}_{2}\right) \delta 7.62-7.42(\mathrm{~m}, 3 \mathrm{H}), 7.41-7.25(\mathrm{~m}, 3 \mathrm{H}), 7.24-6.93(\mathrm{~m}, 3 \mathrm{H})$, $6.92-6.56(\mathrm{~m}, 2 \mathrm{H}), 6.50-6.19(\mathrm{~m}, 1 \mathrm{H}), 6.09-5.88(\mathrm{~m}, 1 \mathrm{H}), 4.84-4.67(\mathrm{~m}, 1 \mathrm{H}), 4.25(\mathrm{~s}, 2 \mathrm{H})$, $4.08-3.95(\mathrm{~m}, 3 \mathrm{H}), 3.87-3.56(\mathrm{~m}, 10 \mathrm{H}), 2.85$ (br s, $1 \mathrm{H}), 2.44-2.25(\mathrm{~m}, 1 \mathrm{H}), 2.05-0.83(\mathrm{~m}$, $22 \mathrm{H})$.

${ }^{13}$ C NMR $\left(151 \mathrm{MHz}, \mathrm{CD}_{2} \mathrm{Cl}_{2}\right) \delta 167.0,166.9,166.9,163.6,162.9,162.8,162.6,161.9,158.3$, $158.3,157.9,154.7,154.7,151.1,150.2,140.2,139.2,138.5,138.3,137.7,137.6,137.3,134.4$, 133.2 , 132.9, 132.1, 131.9, 131.5, 130.9, 130.4, 130.1, 130.0, 130.0, 129.5, 129.1, 128.9, 128.8, $128.6,128.4,128.2,128.1,127.7,127.4,126.6,126.5,126.5,126.4,126.4,125.0,119.8,119.1$, 119.1, 118.8, 118.5, 118.2, 106.3, 104.1, 103.9, 103.4, 72.8, 72.6, 72.5, 72.2, 72.1, 68.9, 64.5, 
$64.5,56.4,56.2,55.5,55.5,55.4,50.0,49.9,48.1,48.1,47.8,42.8,42.4,42.0,38.8,38.6,34.5$, $34.4,30.6,30.5,29.7,29.7,28.5,28.3,28.2,28.0,27.8,27.7,27.6,27.4,27.3,26.9,26.8,26.6$, 26.1, 24.2, 21.8 .

${ }^{31} \mathbf{P}$ NMR $\left(203 \mathrm{MHz}, \mathrm{CD}_{2} \mathrm{Cl}_{2}\right) \delta 46.93,43.72,41.94,40.37$.

IR (neat): 2932, 2849, 1758, 1649, 1589, 1516, 1471, 1428, 1398, 1341, 1303, 1284, 1249, 1218, $1125,1109,1033,998,919,889,851,832,782,728,706 \mathrm{~cm}^{-1}$

M.P.: $197-200{ }^{\circ} \mathrm{C}($ dec.)

EA Calcd. for $\mathrm{C}_{45} \mathrm{H}_{53} \mathrm{ClN}_{3} \mathrm{O}_{7} \mathrm{PPdS}$ : C, 56.72; H, 5.61. Found: C, 56.99; H, 5.88.

\section{(CPhos)Pd(rivaroxaban)Cl (2d)}
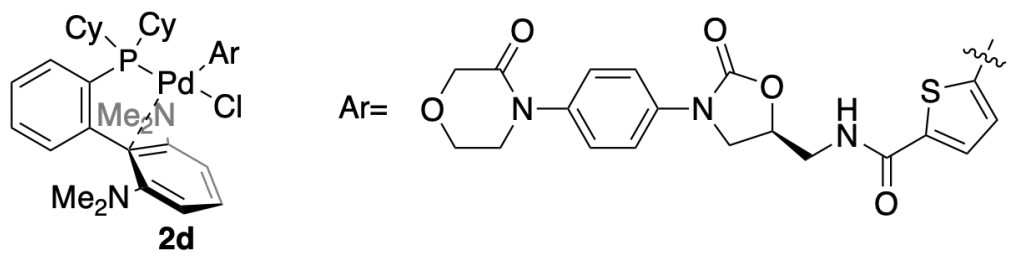

Compound 2d was prepared according to a modified General Procedure $\mathbf{C}$ using ( $t$ BuXPhos)Pd(rivaroxaban)Cl (242 mg, $0.250 \mathrm{mmol}, 1.0$ equiv), CPhos (120 mg, $0.275 \mathrm{mmol}, 1.1$ equiv), and THF (2.5 mL). The precipitate was collected on a fritted funnel and washed with pentane $(25 \mathrm{~mL})$ and $\mathrm{Et}_{2} \mathrm{O}(25 \mathrm{~mL})$ to yield $\mathbf{2 d}(218 \mathrm{mg}, 89 \%)$ as a pale-yellow powder.

${ }^{1} \mathbf{H}$ NMR $\left(500 \mathrm{MHz}, \mathrm{CD}_{2} \mathrm{Cl}_{2}\right) \delta 7.69(\mathrm{t}, J=7.5 \mathrm{~Hz}, 1 \mathrm{H}), 7.60-7.52(\mathrm{~m}, 3 \mathrm{H}), 7.46(\mathrm{t}, J=7.7 \mathrm{~Hz}$, $1 \mathrm{H}), 7.37-7.26(\mathrm{~m}, 4 \mathrm{H}), 7.10-7.00(\mathrm{~m}, 1 \mathrm{H}), 6.84-6.73(\mathrm{~m}, 2 \mathrm{H}), 6.54(\mathrm{~d}, J=3.8 \mathrm{~Hz}, 1 \mathrm{H})$, $4.79(\mathrm{dt}, J=10.3,6.5 \mathrm{~Hz}, 1 \mathrm{H}), 4.26(\mathrm{~s}, 2 \mathrm{H}), 4.08(\mathrm{t}, J=8.9 \mathrm{~Hz}, 1 \mathrm{H}), 4.02-3.96(\mathrm{~m}, 2 \mathrm{H}), 3.85$ $(\mathrm{dd}, J=9.3,6.6 \mathrm{~Hz}, 1 \mathrm{H}), 3.78-3.64(\mathrm{~m}, 4 \mathrm{H}), 3.64-3.54(\mathrm{~m}, 1 \mathrm{H}), 2.61(\mathrm{~d}, J=12.1 \mathrm{~Hz}, 12 \mathrm{H})$, $2.47-2.30(\mathrm{~m}, 2 \mathrm{H}), 2.08(\mathrm{~d}, J=54.8 \mathrm{~Hz}, 2 \mathrm{H}), 1.80-1.61(\mathrm{~m}, 7 \mathrm{H}), 1.59-1.43(\mathrm{~m}, 3 \mathrm{H}), 1.34-$ $1.18(\mathrm{~m}, 4 \mathrm{H}), 1.09(\mathrm{dt}, J=34.3,11.8 \mathrm{~Hz}, 4 \mathrm{H})$.

${ }^{13}$ C NMR $\left(126 \mathrm{MHz}, \mathrm{CD}_{2} \mathrm{Cl}_{2}\right) \delta 167.1,166.9,166.9,162.8,162.5,162.5,158.7,154.8,154.8$, 154.7, 147.5, 147.4, 140.9, 139.6, 139.2, 137.7, 137.6, 137.5, 137.4, 137.4, 137.3, 136.6, 134.5, $134.2,133.9,133.8,133.3,133.3,133.1,133.1,131.9,131.7,130.8,129.0,128.9,128.8,128.5$, $128.1,127.7,126.5,126.4,126.0,119.2,119.0,114.6,114.4,114.3,110.3,72.3,72.2,72.0,68.9$, $64.5,50.0,50.0,48.5,48.4,48.3,45.5,45.1,45.1,45.0,42.8,42.2,37.4,37.2,35.5,35.4,35.1$, $34.6,31.8,31.6,31.3,30.7,30.5,30.5,30.1,30.1,30.0,29.7,28.2$, 28.2, 28.1, 28.1, 27.9, 27.8, 27.8, 27.7, 27.7, 27.6, 26.9, 26.2, 26.2, 24.8, 24.1, 22.7, 21.8.

${ }^{31} \mathbf{P}$ NMR $\left(203 \mathrm{MHz}, \mathrm{CDCl}_{3}\right) \delta 41.00$.

IR (neat): 3335, 3055, 2925, 2852, 2781, 1755, 1649, 1568, 1514, 1476, 1459, 1443, 1426, 1395 , $1343,1305,1284,1215,1161,1125,1076,1043,1007,995,934,922,889,851,832,803,747$, $709,692,645 \mathrm{~cm}^{-1}$

M.P.: $196-200{ }^{\circ} \mathrm{C}$ (dec.)

EA Calcd. for $\mathrm{C}_{47} \mathrm{H}_{59} \mathrm{ClN}_{5} \mathrm{O}_{5} \mathrm{PPdS}$ : C, 57.67; H, 6.08. Found: C, 57.50; H, 6.28. 


\section{(PhXPhos)Pd(rivaroxaban)Cl (2e)}

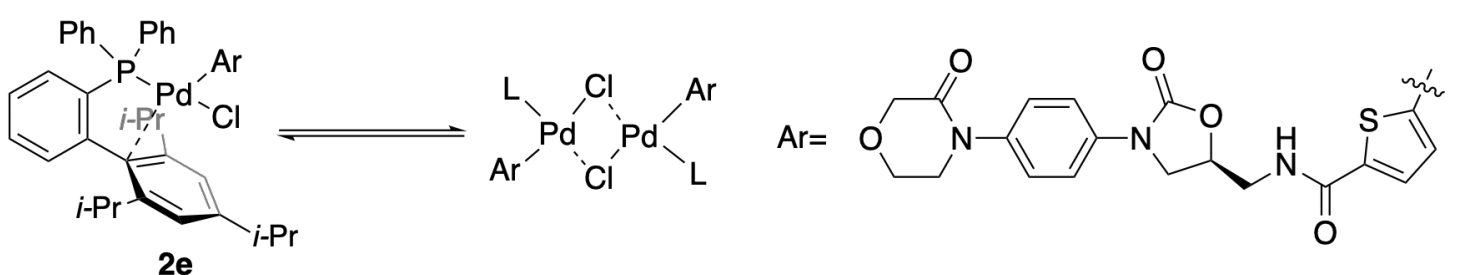

Compound 2e was prepared according to a modified General Procedure $\mathbf{C}$ using ( $t$ BuXPhos)Pd(rivaroxaban)Cl (242 mg, $0.250 \mathrm{mmol}, 1.0$ equiv), $\mathrm{PhXPhos}$ (128 mg, $0.275 \mathrm{mmol}$, 1.1 equiv), and THF $(2.5 \mathrm{~mL})$. The reaction mixture was heated at $60{ }^{\circ} \mathrm{C}$ for $2 \mathrm{~h}$. The precipitate was collected on a fritted funnel to yield $2 \mathrm{e}(227 \mathrm{mg}, 90 \%)$ as a blue-grey powder.

Note: 2e exists both as a monomeric and chloro-bridged dimeric species, as determined by ${ }^{1} \mathrm{H}$ NMR and ${ }^{31} \mathrm{P}$ NMR spectroscopy in $\mathrm{CD}_{2} \mathrm{Cl}_{2}$. In high concentrations $(\sim 30 \mathrm{mg} / \mathrm{mL})$ the dimeric species predominates. When these samples were diluted 10-fold, the monomeric species predominated. ${ }^{31} \mathrm{P}$ NMR spectra of both concentrated and dilute samples are provided.

${ }^{1} \mathbf{H}$ NMR (500 MHz, $\mathrm{CD}_{2} \mathrm{Cl}_{2}$ ) Complicated spectrum, see below.

${ }^{13}$ C NMR $\left(126 \mathrm{MHz}, \mathrm{CD}_{2} \mathrm{Cl}_{2}\right) \delta 167.0,162.8,162.8,162.6,154.8,154.7,154.7,149.2,148.9$, $148.9,146.9,146.7,144.8,144.0,141.2,141.1,141.0,140.8,139.7,137.7,137.7,137.3,135.6$, 135.2 , 134.5, 134.4, 133.8, 133.7, 133.6, 132.3, 132.1, 131.8, 130.8, 130.7, 130.3, 130.2, 130.1, $129.6,129.1,128.9,128.2,128.0,128.0,127.5,127.5,127.5,126.6,126.5,126.5,126.4,125.2$, $120.8,120.7,120.6,119.2,119.1,118.9,72.5,72.4,72.1,68.9,64.5,64.5,50.1,50.0,48.3,48.1$, $47.9,42.8,42.4,42.2,35.1,34.7,34.5,34.3,32.3,30.8,30.6,30.5,26.0,25.9,25.1,24.9,24.2$, 21.9, 21.8, 21.7.

${ }^{31} \mathbf{P}$ NMR $\left(203 \mathrm{MHz}, \mathrm{CD}_{2} \mathrm{Cl}_{2}\right) \delta 41.86,35.76,29.90$.

IR (neat): 3337, 3060, 2960, 2930, 2863, 1758, 1658, 1604, 1587, 1518, 1483, 1466, 1431, 1400, 1379, 1362, 1341, 1308, 1284, 1260, 1239, 1221, 1159, 1123, 1095, 1054, 998, 938, 919, 872, $832,801,780,739,704,690,647 \mathrm{~cm}^{-1}$

M.P.: $184-187^{\circ} \mathrm{C}($ dec.)

EA Calcd. for $\mathrm{C}_{52} \mathrm{H}_{55} \mathrm{ClN}_{3} \mathrm{O}_{5} \mathrm{PPdS}$ : C, 62.03; H, 5.51. Found: C, 62.12; H, 5.66.

\section{(JosiPhos)Pd(rivaroxaban)Cl (2f)}
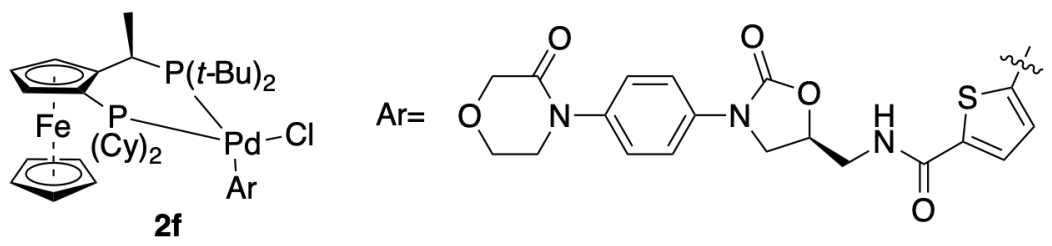

Compound 2f was prepared according to General Procedure C using ( $t$ BuXPhos)Pd(rivaroxaban)Cl (242 mg, $0.250 \mathrm{mmol}, 1.0$ equiv), JosiPhos SL-J009-1 (153 mg, $0.275 \mathrm{mmol}, 1.1$ equiv), and THF $(2.5 \mathrm{~mL})$. The precipitate was collected on a fritted funnel to yield $\mathbf{2 f}(260 \mathrm{mg}, 95 \%)$ as an orange powder. 
${ }^{1}$ H NMR $\left(600 \mathrm{MHz}, \mathrm{CD}_{2} \mathrm{Cl}_{2}\right) \delta 7.61-7.55(\mathrm{~m}, 2 \mathrm{H}), 7.48-7.44(\mathrm{~m}, 1 \mathrm{H}), 7.35-7.28(\mathrm{~m}, 2 \mathrm{H})$, $6.79-6.75(\mathrm{~m}, 1 \mathrm{H}), 6.48(\mathrm{t}, J=6.2 \mathrm{~Hz}, 1 \mathrm{H}), 4.92-4.79(\mathrm{~m}, 2 \mathrm{H}), 4.59(\mathrm{~s}, 1 \mathrm{H}), 4.54(\mathrm{~s}, 1 \mathrm{H}), 4.29$ $(\mathrm{s}, 4 \mathrm{H}), 4.26(\mathrm{~s}, 2 \mathrm{H}), 4.07(\mathrm{t}, J=8.9 \mathrm{~Hz}, 1 \mathrm{H}), 4.02-3.97(\mathrm{~m}, 2 \mathrm{H}), 3.88(\mathrm{dd}, J=9.1,6.7 \mathrm{~Hz}, 1 \mathrm{H})$, $3.78-3.64(\mathrm{~m}, 5 \mathrm{H}), 3.15(\mathrm{p}, J=6.8 \mathrm{~Hz}, 1 \mathrm{H}), 2.65(\mathrm{br} \mathrm{s}, 1 \mathrm{H}), 2.38(\mathrm{q}, J=12.6 \mathrm{~Hz}, 1 \mathrm{H}), 2.27$ (br $\mathrm{s}, 1 \mathrm{H}), 2.03-1.95(\mathrm{~m}, 4 \mathrm{H}), 1.93-1.81(\mathrm{~m}, 4 \mathrm{H}), 1.74(\mathrm{dd}, J=37.1,12.9 \mathrm{~Hz}, 4 \mathrm{H}), 1.63(\mathrm{~d}, J=$ $12.0 \mathrm{~Hz}, 9 \mathrm{H}), 1.55-1.42(\mathrm{~m}, 2 \mathrm{H}), 1.42-1.29(\mathrm{~m}, 5 \mathrm{H}), 1.24(\mathrm{t}, J=13.3 \mathrm{~Hz}, 2 \mathrm{H}), 1.18(\mathrm{~d}, J=$ $13.4 \mathrm{~Hz}, 9 \mathrm{H})$.

${ }^{13}$ C NMR $\left(151 \mathrm{MHz}, \mathrm{CD}_{2} \mathrm{Cl}_{2}\right) \delta 166.9,164.8,163.8,163.1,154.8,139.3,139.3,139.3,139.3$, 137.6, 137.4, 126.4, 126.4, 119.1, 119.1, 119.1, 97.5, 97.4, 97.4, 97.3, 72.9, 72.9, 72.4, 72.4, $72.3,72.1,72.1,70.2,70.0,69.9,69.8,69.4,69.4,68.9,64.5,50.0,48.3,42.4,39.4,39.4,38.5$, $38.5,33.1,33.1,33.0,32.2,32.2,31.2,31.2$, 28.2, 27.8, 27.7, 27.6, 27.6, 27.5, 27.4, 27.3, 27.1, 27.0, 26.5, 26.1, 17.8, 17.8.

${ }^{31}$ P NMR $\left(203 \mathrm{MHz}, \mathrm{CD}_{2} \mathrm{Cl}_{2}\right) \delta 81.93(\mathrm{~d}, J=22.1 \mathrm{~Hz}), 26.30$.

IR (neat): 3330, 3088, 2925, 2854, 1755, 1663, 1642, 1514, 1481, 1445, 1391, 1343, 1305, 1267, $1241,1215,1173,1154,1123,1109,1047,998,919,889,827,810,749,737,706,687 \mathrm{~cm}^{-1}$

M.P.: $222-224^{\circ} \mathrm{C}($ dec.)

EA Calcd. for $\mathrm{C}_{51} \mathrm{H}_{70} \mathrm{ClFeN}_{3} \mathrm{O}_{5} \mathrm{P}_{2} \mathrm{PdS}$ : C, 55.85; H, 6.43. Found: C, 56.12; H, 6.64.

\section{(XantPhos)Pd(rivaroxaban)Cl (2g)}
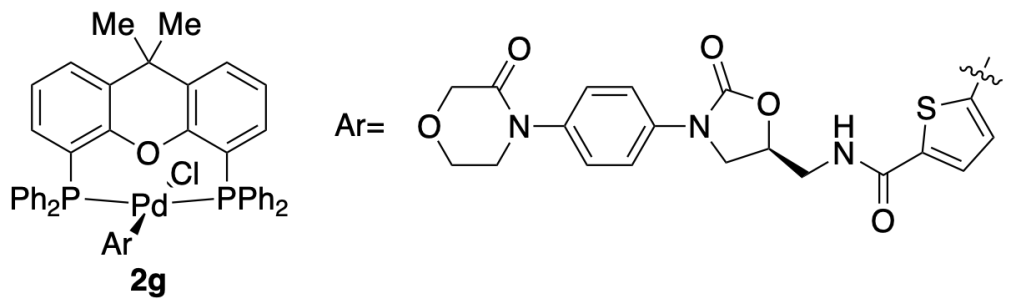

Compound $\mathbf{2 g}$ was prepared according to a modified General Procedure $\mathbf{C}$ using ( $t$ BuXPhos)Pd(rivaroxaban)Cl (242 mg, $0.250 \mathrm{mmol}, 1$ equiv), XantPhos (159 mg, $0.275 \mathrm{mmol}$, 1.1 equiv), and THF $(2.5 \mathrm{~mL})$. The precipitate was collected on a fritted funnel and washed with pentane $(25 \mathrm{~mL})$ and $\mathrm{Et}_{2} \mathrm{O}(25 \mathrm{~mL})$ to yield $\mathbf{2 g}(257 \mathrm{mg}, 92 \%)$ as a pale-yellow powder.

${ }^{1} \mathbf{H}$ NMR (500 MHz, $\mathrm{CD}_{2} \mathrm{Cl}_{2}$ ) Complex spectrum, see below.

${ }^{13}$ C NMR $\left(126 \mathrm{MHz}, \mathrm{CD}_{2} \mathrm{Cl}_{2}\right) \delta 166.9,163.0,156.9,156.8,155.9,155.9,155.8,154.7,138.3$, $137.8,137.3,135.5,135.5,135.5,132.7,131.6,131.6,131.5,130.1,128.5,128.1,126.5,124.8$, $124.8,124.8,121.3,121.2,121.0,119.0,72.6,68.9,64.5,50.0,48.0,42.3,36.7$.

${ }^{31} \mathbf{P}$ NMR $\left(203 \mathrm{MHz}, \mathrm{CD}_{2} \mathrm{Cl}_{2}\right) \delta 9.50$.

IR (neat): 3344, 3058, 2968, 1753, 1658, 1649, 1521, 1481, 1433, 1398, 1341, 1308, 1284, 1239 , $1213,1159,1125,1095,1066,1026,995,922,870,832,792,780,742,694 \mathrm{~cm}^{-1}$

M.P.: $216-218^{\circ} \mathrm{C}$ (dec.)

HRMS (ESI) Calcd. For $\mathrm{C}_{58} \mathrm{H}_{50} \mathrm{~N}_{3} \mathrm{O}_{6} \mathrm{P}_{2} \mathrm{PdS}(\mathrm{M}-\mathrm{Cl})^{+}$: 1084.1925. Found: 1084.1934. 


\section{(dtbbpy)Pd(rivaroxaban)Cl (2h)}

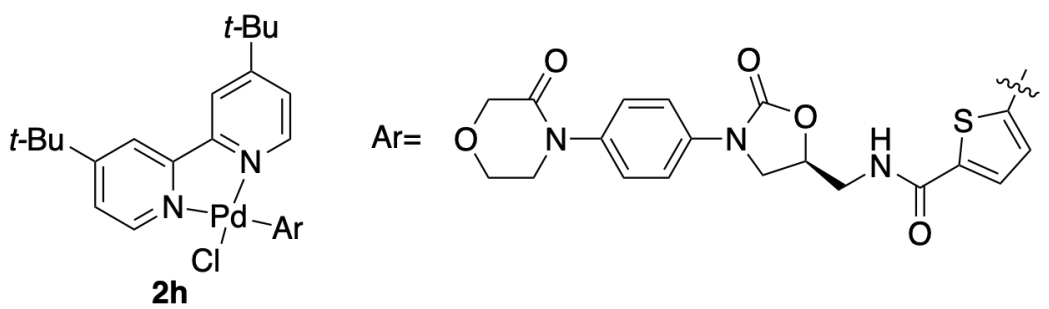

Compound $\mathbf{2 h}$ was prepared according to General Procedure $\mathbf{C}$ using ( $t$ BuXPhos)Pd(rivaroxaban)Cl (242 mg, $0.250 \mathrm{mmol}, 1$ equiv), dtbbpy ( $74 \mathrm{mg}, 0.275 \mathrm{mmol}, 1.1$ equiv), and THF $(2.5 \mathrm{~mL})$. The precipitate was collected on a fritted funnel to yield $\mathbf{2 h}(182 \mathrm{mg}$, $90 \%$ ) as an off-white powder.

${ }^{1} \mathbf{H}$ NMR $\left(600 \mathrm{MHz}, \mathrm{CD}_{2} \mathrm{Cl}_{2}\right) \delta 9.05(\mathrm{~d}, J=5.8 \mathrm{~Hz}, 1 \mathrm{H}), 8.03(\mathrm{~d}, J=2.0 \mathrm{~Hz}, 2 \mathrm{H}), 7.90(\mathrm{~d}, J=$ $6.1 \mathrm{~Hz}, 1 \mathrm{H}), 7.60(\mathrm{dd}, J=5.8,1.9 \mathrm{~Hz}, 1 \mathrm{H}), 7.58-7.50(\mathrm{~m}, 3 \mathrm{H}), 7.36(\mathrm{dd}, J=6.1,2.1 \mathrm{~Hz}, 1 \mathrm{H})$, $7.32-7.26(\mathrm{~m}, 2 \mathrm{H}), 6.87(\mathrm{~d}, J=3.6 \mathrm{~Hz}, 1 \mathrm{H}), 6.75(\mathrm{t}, J=6.1 \mathrm{~Hz}, 1 \mathrm{H}), 4.86(\mathrm{ddt}, J=8.8,6.7,5.4$ $\mathrm{Hz}, 1 \mathrm{H}), 4.26(\mathrm{~s}, 2 \mathrm{H}), 4.11(\mathrm{t}, J=8.9 \mathrm{~Hz}, 1 \mathrm{H}), 4.02-3.97(\mathrm{~m}, 2 \mathrm{H}), 3.91(\mathrm{dd}, J=9.1,6.7 \mathrm{~Hz}$, $1 \mathrm{H}), 3.81-3.69(\mathrm{~m}, 4 \mathrm{H}), 1.43(\mathrm{~s}, 9 \mathrm{H}), 1.38(\mathrm{~s}, 9 \mathrm{H})$.

${ }^{13}$ C NMR $\left(151 \mathrm{MHz}, \mathrm{CD}_{2} \mathrm{Cl}_{2}\right) \delta 166.9,164.9,164.6,163.0,156.8,154.8,154.5,152.5,151.7$, 149.1, 139.6, 137.6, 137.3, 130.4, 129.8, 126.5, 124.5, 124.2, 119.6, 119.1, 118.9, 72.4, 68.9, $64.5,50.0,48.3,42.5,36.0,35.9,30.4,30.3$.

IR (neat): 3337, 3065, 2965, 2911, 2878, 1746, 1649, 1616, 1514, 1476, 1398, 1341, 1308, 1291, $1249,1218,1123,1076,1033,1024,995,922,898,834,803,744,718,706,685,602 \mathrm{~cm}^{-1}$

M.P.: $222-224^{\circ} \mathrm{C}$ (dec.)

HRMS (ESI) Calcd. For $\mathrm{C}_{37} \mathrm{H}_{42} \mathrm{~N}_{5} \mathrm{O}_{5} \mathrm{PdS}(\mathrm{M}-\mathrm{Cl})^{+}$: 774.1936. Found: 774.1955.

\section{$\left(\mathrm{PMe}_{3}\right)_{2} \mathbf{P d}($ rivaroxaban $) \mathrm{Cl}(2 \mathrm{i})$}
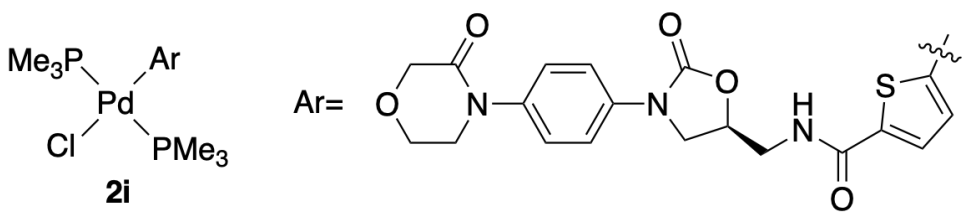

Compound 2i was prepared according to a modified General Procedure C. An oven-dried $16 \mathrm{~mL}$ reaction tube was equipped, while still hot, with a magnetic stir bar and septum cap. The tube was allowed to cool to room temperature under vacuum by piercing the septum with a needle connected to a dual manifold by a rubber hose. At this point, the cap was removed and the tube was charged with ( $t$-BuXPhos)Pd(rivaroxaban)Cl (97 mg, $0.100 \mathrm{mmol}, 1.0$ equiv). The tube was recapped, the septum was pierced with a needle and reconnected to the dual manifold by a rubber hose. The tube was evacuated and backfilled with nitrogen (the evacuation/backfill process was repeated a total of three times). Under nitrogen, THF $(1.0 \mathrm{~mL})$ and $\mathrm{PMe}_{3}(1.0 \mathrm{M}, 240 \mu \mathrm{L}, 2.4$ equiv) were added to the tube sequentially via syringe. The reaction mixture was allowed to stir at room temperature for $30 \mathrm{~min}$. Over the course of the reaction, a pale-blue-grey powder precipitated. At this time, the reaction tube was opened to air and the reaction mixture was pipetted dropwise into a $20 \mathrm{~mL}$ scintillation vial containing $10 \mathrm{~mL}$ of pentane to assist with 
precipitation. The precipitate was collected on a fritted funnel and washed with pentane $(10 \mathrm{~mL})$ to yield $\mathbf{2 i}$ (64 $\mathrm{mg}, 92 \%$ ) as a pale-blue-grey powder.

${ }^{1} \mathbf{H}$ NMR $\left(600 \mathrm{MHz}, \mathrm{CD}_{2} \mathrm{Cl}_{2}\right) \delta 7.59-7.53(\mathrm{~m}, 2 \mathrm{H}), 7.49(\mathrm{~d}, J=3.6 \mathrm{~Hz}, 1 \mathrm{H}), 7.37-7.30(\mathrm{~m}$, $2 \mathrm{H}), 6.71(\mathrm{~d}, J=3.6 \mathrm{~Hz}, 1 \mathrm{H}), 6.43(\mathrm{t}, J=6.2 \mathrm{~Hz}, 1 \mathrm{H}), 4.84(\mathrm{dtd}, J=8.8,6.4,3.7 \mathrm{~Hz}, 1 \mathrm{H}), 4.26$ $(\mathrm{s}, 2 \mathrm{H}), 4.09(\mathrm{t}, J=8.9 \mathrm{~Hz}, 1 \mathrm{H}), 4.02-3.98(\mathrm{~m}, 2 \mathrm{H}), 3.85(\mathrm{dd}, J=9.1,6.7 \mathrm{~Hz}, 1 \mathrm{H}), 3.80$ (ddd, $J$ $=14.6,6.3,3.7 \mathrm{~Hz}, 1 \mathrm{H}), 3.73-3.70(\mathrm{~m}, 2 \mathrm{H}), 3.70-3.64(\mathrm{~m}, 1 \mathrm{H}), 1.21(\mathrm{t}, J=3.7 \mathrm{~Hz}, 18 \mathrm{H})$.

${ }^{13}$ C NMR $\left(151 \mathrm{MHz}, \mathrm{CD}_{2} \mathrm{Cl}_{2}\right) \delta 166.9,162.9,155.3,155.2,155.1,154.7,139.9,139.8,139.8$, $137.8,137.2,130.8,130.7,130.7,130.2,126.5,119.1,72.6,68.9,64.5,50.0,48.1,42.6,13.4$, 13.3, 13.2 .

${ }^{31} \mathbf{P}$ NMR $\left(202 \mathrm{MHz}, \mathrm{CD}_{2} \mathrm{Cl}_{2}\right) \delta-13.76$.

IR (neat): 3429, 3408, 2975, 2904, 1736, 1653, 1634, 1516, 1497, 1488, 1473, 1348, 1398, 1343, 1312, 1303, 1293, 1241, 1230, 1147, 1135, 1123, 1099, 1087, 1052, 1035, 1002, 993, 948, 924, $872,855,820,806,777,744,704,690,666 \mathrm{~cm}^{-1}$

M.P.: $231-233^{\circ} \mathrm{C}$ (dec.)

EA Calcd. for $\mathrm{C}_{25} \mathrm{H}_{36} \mathrm{ClN}_{3} \mathrm{O}_{5} \mathrm{P}_{2} \mathrm{PdS}$ : C, 43.24; H, 5.23. Found: C, 43.43; H, 5.26.

\section{$\left[\mathbf{P}(\mathrm{OMe})_{3}\right]_{2} \mathbf{P d}($ rivaroxaban $) \mathrm{Cl}(\mathbf{2 j})$}
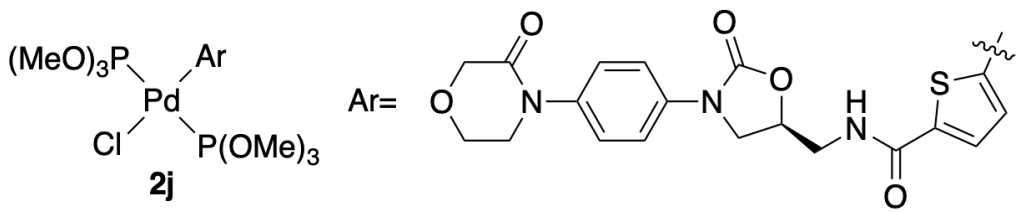

Compound $\mathbf{2} \mathbf{j}$ was prepared according to a modified General Procedure $\mathbf{C}$. An oven-dried $16 \mathrm{~mL}$ reaction tube was equipped, while still hot, with a magnetic stir bar and septum cap. The tube was allowed to cool to room temperature under vacuum by piercing the septum with a needle connected to a dual manifold by a rubber hose. At this point, the cap was removed and the tube was charged with ( $t$-BuXPhos)Pd(rivaroxaban)Cl ( $97 \mathrm{mg}, 0.100 \mathrm{mmol}, 1.0$ equiv). The tube was recapped, the septum was pierced with a needle and reconnected to the dual manifold by a rubber hose. The tube was evacuated and backfilled with nitrogen (the evacuation/backfill process was repeated a total of three times). Under nitrogen, THF $(1.0 \mathrm{~mL})$ and $\mathrm{P}(\mathrm{OMe})_{3}(28 \mu \mathrm{L}, 0.24 \mathrm{mmol}$, 2.4 equiv) were added to the tube sequentially via syringe. The reaction mixture was allowed to stir at room temperature for $30 \mathrm{~min}$. Over the course of the reaction, a light-grey powder precipitated. At this time, the reaction tube was opened to air and the reaction mixture was pipetted dropwise into a $20 \mathrm{~mL}$ scintillation vial containing $10 \mathrm{~mL}$ of pentane to assist with precipitation. The precipitate was collected on a fritted funnel and washed with pentane $(10 \mathrm{~mL})$ to yield $\mathbf{2} \mathbf{j}$ (66 $\mathrm{mg}, 84 \%$ ) as a light-grey powder.

Note: Compound $\mathbf{2} \mathbf{j}$ was found to be thermally unstable and showed significant decomposition after 2 days in air and in vacuo by ${ }^{1} \mathrm{H}$ and ${ }^{31} \mathrm{P}$ spectroscopic analysis. The ${ }^{31} \mathrm{P}$ NMR spectrum was obtained with a dilute sample of $\mathbf{2} \mathbf{j}$, while the ${ }^{1} \mathrm{H}$ and ${ }^{13} \mathrm{C}$ NMR spectra were obtained with a more concentrated sample.

${ }^{1} \mathbf{H}$ NMR $\left(500 \mathrm{MHz}, \mathrm{CD}_{2} \mathrm{Cl}_{2}\right) \delta 7.60-7.51(\mathrm{~m}, 2 \mathrm{H}), 7.49(\mathrm{~d}, J=3.8 \mathrm{~Hz}, 1 \mathrm{H}), 7.36-7.29(\mathrm{~m}$, 2H), $6.77(\mathrm{~d}, J=3.7 \mathrm{~Hz}, 1 \mathrm{H}), 6.46(\mathrm{t}, J=6.4 \mathrm{~Hz}, 1 \mathrm{H}), 4.84(\mathrm{dtd}, J=8.7,6.4,3.9 \mathrm{~Hz}, 1 \mathrm{H}), 4.26$ 
(s, 2H), $4.09(\mathrm{t}, J=8.9 \mathrm{~Hz}, 1 \mathrm{H}), 4.00(\mathrm{dd}, J=6.0,4.1 \mathrm{~Hz}, 2 \mathrm{H}), 3.89-3.79(\mathrm{~m}, 2 \mathrm{H}), 3.79-3.70$ (m, 3H), $3.66(\mathrm{~s}, 18 \mathrm{H})$.

${ }^{13}$ C NMR $\left(126 \mathrm{MHz}, \mathrm{CD}_{2} \mathrm{Cl}_{2}\right) \delta 167.0,162.9,154.7,140.3,137.8,137.3,132.8,129.4,126.6$, $126.5,119.2,72.5,72.0,68.9,64.5,53.0,50.0,48.2,43.0,42.7$.

${ }^{31} \mathbf{P}$ NMR $\left(202 \mathrm{MHz}, \mathrm{CD}_{2} \mathrm{Cl}_{2}\right) \delta 108.95$.

IR (neat): 3410, 2956, 2894, 2842, 1734, 1653, 1632, 1516, 1485, 1473, 1438, 1400, 1346, 1310, 1301, 1291, 1277, 1241, 1222, 1177, 1144, 1123, 1099, 1085, 1009, 943, 934, 929, 872, 822, $796,775,744,706 \mathrm{~cm}^{-1}$

M.P.: $111-115^{\circ} \mathrm{C}(\mathrm{dec}$.

EA Calcd. for $\mathrm{C}_{25} \mathrm{H}_{36} \mathrm{ClN}_{3} \mathrm{O}_{11} \mathrm{P}_{2} \mathrm{PdS}$ : C, 37.99; H, 4.59. Found: $\mathrm{C}, 38.10 ; \mathrm{H}, 4.58$.

$\left[\mathrm{P}(\mathrm{OPh})_{3}\right]_{2} \mathrm{Pd}($ rivaroxaban $) \mathrm{Cl}(\mathbf{2 k})$
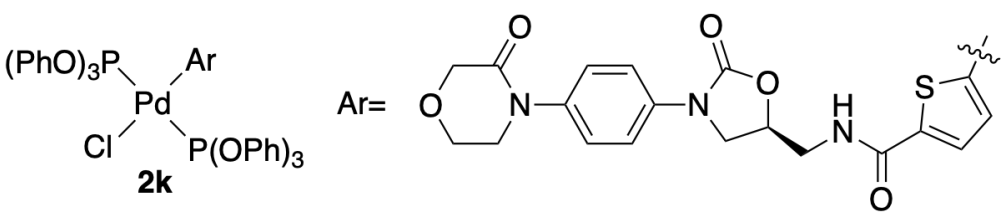

Compound 2k was prepared according to a modified General Procedure C. An oven-dried $16 \mathrm{~mL}$ reaction tube was equipped, while still hot, with a magnetic stir bar and septum cap. The tube was allowed to cool to room temperature under vacuum by piercing the septum with a needle connected to a dual manifold by a rubber hose. At this point, the cap was removed and the tube was charged with ( $t$-BuXPhos)Pd(rivaroxaban) $\mathrm{Cl}$ ( $48 \mathrm{mg}, 0.050 \mathrm{mmol}, 1.0$ equiv). The tube was recapped, the septum was pierced with a needle connected to the dual manifold by a rubber hose. The tube was evacuated and backfilled with nitrogen (the evacuation/backfill process was repeated a total of three times). Under nitrogen, THF $(1.0 \mathrm{~mL})$ and $\mathrm{P}(\mathrm{OPh})_{3}(31 \mu \mathrm{L}, 0.12 \mathrm{mmol}$, 2.4 equiv) were added sequentially via syringe. The reaction mixture was allowed to stir at room temperature for $30 \mathrm{~min}$. At this time, the reaction tube was opened to air and the reaction mixture was pipetted dropwsie into a $20 \mathrm{~mL}$ scintillation vial containing $10 \mathrm{~mL}$ of pentane to assist with precipitation. The precipitate was collected on a fritted funnel and washed with pentane $(10 \mathrm{~mL})$ to yield $2 \mathbf{k}(39 \mathrm{mg}, 68 \%)$ as a white powder.

${ }^{1} \mathbf{H}$ NMR $\left(600 \mathrm{MHz}, \mathrm{CD}_{2} \mathrm{Cl}_{2}\right) \delta 7.60-7.53(\mathrm{~m}, 2 \mathrm{H}), 7.39-7.30(\mathrm{~m}, 3 \mathrm{H}), 7.30-7.13(\mathrm{~m}, 18 \mathrm{H})$, $7.07(\mathrm{~d}, J=8.0 \mathrm{~Hz}, 10 \mathrm{H}), 6.90(\mathrm{~d}, J=8.2 \mathrm{~Hz}, 1 \mathrm{H}), 6.17(\mathrm{t}, J=6.3 \mathrm{~Hz}, 1 \mathrm{H}), 5.81(\mathrm{~d}, J=3.7 \mathrm{~Hz}$, $1 \mathrm{H}), 4.83$ (dtd, $J=8.7,6.6,3.5 \mathrm{~Hz}, 1 \mathrm{H}), 4.26$ (s, 2H), 4.09 (t, $J=8.9 \mathrm{~Hz}, 1 \mathrm{H}), 4.00-3.95$ (m, $2 \mathrm{H}), 3.86-3.79(\mathrm{~m}, 2 \mathrm{H}), 3.73-3.62(\mathrm{~m}, 3 \mathrm{H})$.

${ }^{13}$ C NMR $\left(151 \mathrm{MHz}, \mathrm{CD}_{2} \mathrm{Cl}_{2}\right) \delta 166.9,162.6,154.7,150.7,150.7,150.7,142.4,140.8,137.9$, $137.2,132.6,132.5,132.5,130.4,130.3,130.2,129.4,126.5,126.1,125.9,125.8,121.5,121.4$, $120.9,120.9,120.8,120.6,120.5,119.1,119.1,72.5,68.9,64.5,50.0,48.1,42.6$.

${ }^{31} \mathbf{P}$ NMR $\left(203 \mathrm{MHz}, \mathrm{CD}_{2} \mathrm{Cl}_{2}\right) \delta 93.24$.

IR (neat): $3337,3067,2868,1753,1658,1639,1587,1516,1485,1455,1426,1400,1376,1343$, $1310,1284,1211,1175,1151,1125,1069,1021,1005,990,912,832,806,747,720,709,685$, $616,590 \mathrm{~cm}^{-1}$

M.P.: $115-120^{\circ} \mathrm{C}($ dec. $)$

EA Calcd. for $\mathrm{C}_{55} \mathrm{H}_{48} \mathrm{ClN}_{3} \mathrm{O}_{11} \mathrm{P}_{2} \mathrm{PdS}$ : C, 56.81; H, 4.16. Found: C, 56.65; H, 4.23. 
(RuPhos)Pd(X4)Br (3)

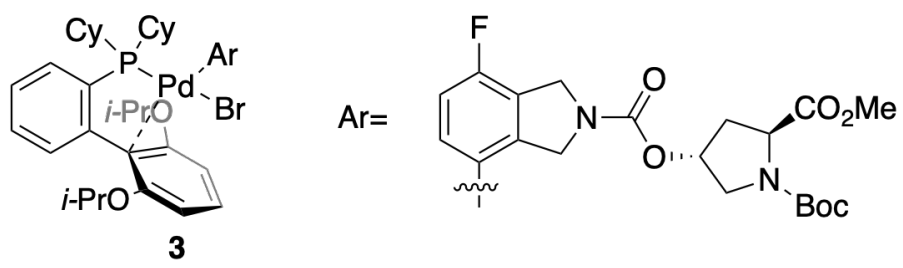

Compound 3 was prepared according to a modified General Procedure $\mathbf{C}$ using ( $t$ BuXPhos)Pd(X4)Br (255 mg, $0.250 \mathrm{mmol}, 1$ equiv), RuPhos (128 mg, $0.275 \mathrm{mmol}, 1.1$ equiv), and THF $(2.5 \mathrm{~mL})$. The reaction mixture was allowed to stir for $2 \mathrm{~h}$ at $60{ }^{\circ} \mathrm{C}$. Following filtration through Celite ${ }^{\circledR}$ and concentrating in vacuo with the assistance of a rotary evaporator, the resulting residue was suspended in pentane $(25 \mathrm{~mL})$ and sonicated for $\sim 10 \mathrm{~min}$ to form a grey powder. The powder was collected on a fritted funnel and washed with pentane $(25 \mathrm{~mL})$ to yield 3 (196 mg, 74\%) as a grey powder. Note: Compound 3 is mixture of rotamers.

${ }^{1}$ H NMR $\left(600 \mathrm{MHz}, \mathrm{CD}_{2} \mathrm{Cl}_{2}\right) \delta 7.72-7.65(\mathrm{~m}, 1 \mathrm{H}), 7.59(\mathrm{q}, J=7.0 \mathrm{~Hz}, 1 \mathrm{H}), 7.44(\mathrm{td}, J=7.5$, $1.8 \mathrm{~Hz}, 1 \mathrm{H}), 7.38(\mathrm{td}, J=7.7,4.2 \mathrm{~Hz}, 1 \mathrm{H}), 6.84-6.68(\mathrm{~m}, 3 \mathrm{H}), 6.66-6.49(\mathrm{~m}, 2 \mathrm{H}), 5.28-5.18$ $(\mathrm{m}, 1 \mathrm{H}), 4.85-4.74(\mathrm{~m}, 2 \mathrm{H}), 4.67(\mathrm{dd}, J=19.9,4.8 \mathrm{~Hz}, 2 \mathrm{H}), 4.64-4.50(\mathrm{~m}, 2 \mathrm{H}), 4.42-4.28$ $(\mathrm{m}, 1 \mathrm{H}), 3.90-3.45(\mathrm{~m}, 5 \mathrm{H}), 2.49-2.33(\mathrm{~m}, 2 \mathrm{H}), 2.28-2.06(\mathrm{~m}, 3 \mathrm{H}), 1.88-1.73(\mathrm{~m}, 4 \mathrm{H})$, $1.73-1.59(\mathrm{~m}, 4 \mathrm{H}), 1.55-1.42(\mathrm{~m}, 7 \mathrm{H}), 1.42-1.38(\mathrm{~m}, 5 \mathrm{H}), 1.36(\mathrm{~s}, 2 \mathrm{H}), 1.31(\mathrm{dd}, J=6.1,4.5$ $\mathrm{Hz}, 3 \mathrm{H}), 1.27-1.12(\mathrm{~m}, 6 \mathrm{H}), 1.11-1.07(\mathrm{~m}, 4 \mathrm{H}), 1.02-0.85(\mathrm{~m}, 4 \mathrm{H}), 0.49-0.18(\mathrm{~m}, 1 \mathrm{H})$.

${ }^{13}$ C NMR $\left(151 \mathrm{MHz}, \mathrm{CD}_{2} \mathrm{Cl}_{2}\right) \delta 173.7,173.6,173.5,173.4,173.1,173.1,162.1,162.0,161.9$, $161.3,160.5,160.4,160.3,160.1,157.6,157.5,156.0,155.9,154.6,154.3,154.3,154.0,153.9$, $153.8,153.6,146.9,145.8,145.7,145.7,145.6,145.5,145.4,136.8,136.7,136.6,136.2,136.2$, $135.4,135.1,135.0,134.9,134.4,134.1,132.8,132.7,132.6,132.5,132.4,131.5,131.4,131.3$, $131.2,131.2,131.1,131.1,128.1,127.0,126.9,126.9,126.9,125.9,124.0,123.9,123.9,123.8$, $121.6,121.5,121.3,121.2,120.8,113.5,113.3,113.2,113.1,109.6,108.8,108.4,107.7,107.4$, 107.3, 107.2, 107.0, 106.8, 106.7, 106.7, 106.6, 80.5, 80.5, 80.4, 80.4, 73.8, 73.7, 73.6, 73.5, $73.3,73.0,72.3,72.3,71.6,71.6,71.5,71.3,71.0,70.8,70.7,58.5,58.5,58.4,58.4,58.3,58.2$, 58.1, 57.6, 53.1, 53.1, 53.0, 52.8, 52.8, 52.7, 52.6, 52.4, 52.4, 50.9, 50.8, 50.8, 50.2, 37.4, 37.4, $37.3,36.4,36.3,36.0,35.3,35.2,35.1,35.1,35.1,35.0,34.9,32.7,32.7,32.6,32.6,32.5,31.2$, $31.1,30.8,30.7,30.6,29.7,29.6,29.6,29.5,28.5,28.5,28.4,28.3,28.3,27.6,27.6,27.6,27.5$, $27.5,27.4,27.1,27.0,26.9,26.9,26.8,26.8,26.7,26.6,26.5,26.5,26.4,26.2,26.0,25.8,25.8$, 25.8, 24.2, 22.9, 22.8, 22.7, 22.7, 22.7, 22.1, 22.1, 22.0, 22.0, 21.9, 21.9, 21.8.

${ }^{19}$ F NMR $\left(565 \mathrm{MHz}, \mathrm{CD}_{2} \mathrm{Cl}_{2}\right) \delta-130.29,-130.32,-130.35,-130.55(\mathrm{~d}, J=12.7 \mathrm{~Hz}),-130.66$ $(\mathrm{d}, J=11.4 \mathrm{~Hz})$.

${ }^{31}$ P NMR (203 MHz, $\left.\mathrm{CD}_{2} \mathrm{Cl}_{2}\right) \delta 34.68,34.49,34.09(\mathrm{~d}, J=16.2 \mathrm{~Hz}), 33.36,33.21$ (d, $J=7.6$ $\mathrm{Hz})$.

IR (neat): 3055, 2977, 2927, 2854, 1751, 1706, 1589, 1571, 1452, 1419, 1395, 1362, 1329, 1244, $1201,1177,1163,1109,1064,993,926,896,872,860,848,803,763,732,697,675 \mathrm{~cm}^{-1}$

M.P.: $217-219^{\circ} \mathrm{C}($ dec.)

HRMS (ESI) Calcd. For $\mathrm{C}_{50} \mathrm{H}_{67} \mathrm{FN}_{2} \mathrm{O}_{8} \mathrm{PPd}(\mathrm{M}-\mathrm{Br})^{+}$: 979.3648 . Found: 979.3663. 


\section{(RuPhos)Pd(X14)I (4)}

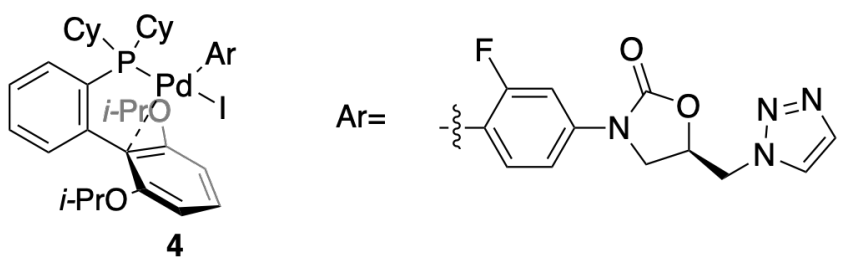

Compound 4 was prepared according to General Procedure C using S9 (230 mg, 0.250 mmol, 1 equiv), RuPhos (128 mg, $0.275 \mathrm{mmol}, 1.1$ equiv), and THF (2.5 mL). The precipitate was collected on a fritted funnel to yield $4(214 \mathrm{mg}, 89 \%)$ as a pale-yellow powder. Note: Compound 4 is a 1:1 mixture of atropisomers

${ }^{1}$ H NMR $\left(600 \mathrm{MHz}, \mathrm{CD}_{2} \mathrm{Cl}_{2}\right) \delta 7.80(\mathrm{~s}, 1 \mathrm{H}), 7.71-7.60(\mathrm{~m}, 2 \mathrm{H}), 7.41(\mathrm{dt}, J=38.7,7.5 \mathrm{~Hz}, 2 \mathrm{H})$, 6.91 (ddt, $J=23.5,8.7,5.5 \mathrm{~Hz}, 2 \mathrm{H}), 6.80(\mathrm{dd}, J=7.8,3.1 \mathrm{~Hz}, 1 \mathrm{H}), 6.66(\mathrm{~d}, J=8.4 \mathrm{~Hz}, 1 \mathrm{H}), 6.57$ $(\mathrm{d}, J=8.3 \mathrm{~Hz}, 1 \mathrm{H}), 5.00(\mathrm{dd}, J=5.9,3.3 \mathrm{~Hz}, 1 \mathrm{H}), 4.77(\mathrm{dd}, J=14.6,4.0 \mathrm{~Hz}, 1 \mathrm{H}), 4.70(\mathrm{ddd}, J=$ 14.7, 6.0, 3.2 Hz, 1H), $4.62-4.52(\mathrm{~m}, 2 \mathrm{H}), 4.10$ (q, $J=8.9 \mathrm{~Hz}, 1 \mathrm{H}), 3.78$ (ddd, $J=9.4,6.3,2.9$ $\mathrm{Hz}, 1 \mathrm{H}), 2.20(\mathrm{dq}, J=28.1,9.7,7.1 \mathrm{~Hz}, 2 \mathrm{H}), 2.07(\mathrm{q}, J=11.3 \mathrm{~Hz}, 1 \mathrm{H}), 1.76(\mathrm{~d}, J=13.4 \mathrm{~Hz}$, $5 \mathrm{H}), 1.72-1.63(\mathrm{~m}, 3 \mathrm{H}), 1.62-1.54(\mathrm{~m}, 2 \mathrm{H}), 1.49-1.39(\mathrm{~m}, 4 \mathrm{H}), 1.34(\mathrm{~d}, J=5.9 \mathrm{~Hz}, 4 \mathrm{H})$, $1.26-1.12(\mathrm{~m}, 6 \mathrm{H}), 1.07(\mathrm{~d}, J=5.9 \mathrm{~Hz}, 4 \mathrm{H}), 1.00(\mathrm{ddd}, J=12.5,8.5,4.5 \mathrm{~Hz}, 1 \mathrm{H}), 0.94(\mathrm{~d}, J=$ $6.1 \mathrm{~Hz}, 3 \mathrm{H}$ ), 0.57 (ddd, $J=18.8,12.1,6.7 \mathrm{~Hz}, 1 \mathrm{H}$ ).

${ }^{13}$ C NMR $\left(151 \mathrm{MHz}, \mathrm{CD}_{2} \mathrm{Cl}_{2}\right) \delta 164.8,163.3,161.7,160.5,160.5,153.6,153.6,145.3,145.1$, $138.8,138.8,138.7,136.3,136.0,135.9,134.5,134.4,134.3,132.8,132.7,131.3,131.3,131.2$, $126.8,126.7,125.4,113.4,109.0,108.2,107.5,107.3,107.2,104.6,104.6,104.4,104.3,72.2$, 72.2, 71.0, 70.9, 70.9, 52.6, 52.6, 47.9, 47.8, 35.9, 35.7, 33.6, 33.6, 33.4, 33.4, 31.4, 31.3, 31.1, $31.0,29.4,29.4,29.4,29.3,28.8,27.9,27.9,27.6,27.5,27.4,27.3,27.3,27.2$, 27.1, 27.0, 26.6, 26.6, 26.5, 26.3, 22.3, 22.0, 21.9, 21.7.

${ }^{19}$ F NMR $\left(565 \mathrm{MHz}, \mathrm{CD}_{2} \mathrm{Cl}_{2}\right) \delta-83.68,-83.79$.

${ }^{31} \mathbf{P}$ NMR $\left(203 \mathrm{MHz}, \mathrm{CD}_{2} \mathrm{Cl}_{2}\right) \delta 31.18,31.16,31.14,31.11$.

IR (neat): 2975, 2925, 2849, 1758, 1587, 1566, 1471, 1452, 1412, 1381, 1369, 1317, 1270, 1246, $1225,1185,1109,1061,1035,1002,938,919,898,848,808,785,765,744,697,675 \mathrm{~cm}^{-1}$

M.P.: $191-194^{\circ} \mathrm{C}$ (dec.)

EA Calcd. for $\mathrm{C}_{42} \mathrm{H}_{53} \mathrm{FIN}_{4} \mathrm{O}_{4} \mathrm{PPd}$ : C, 52.85; H, 5.88. Found: C, 53.10; H, 5.64.

\section{(RuPhos)Pd(Indometacin)Cl (5)}
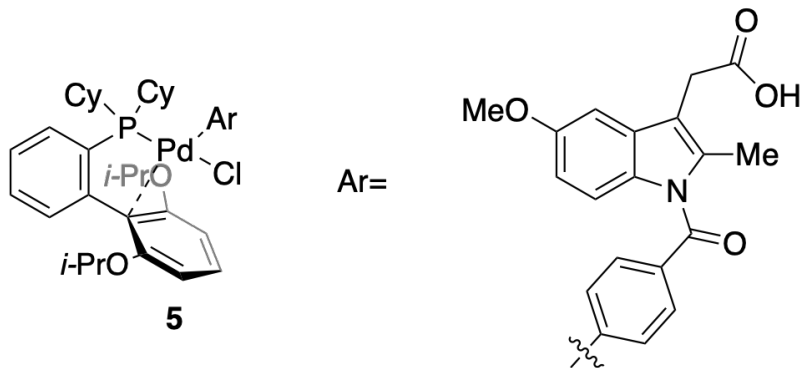
Compound 5 was prepared according to General Procedure C using S10 (219 mg, $0.250 \mathrm{mmol}, 1$ equiv), RuPhos (128 mg, $0.275 \mathrm{mmol}, 1.1$ equiv), and THF $(2.5 \mathrm{~mL})$. The precipitate was collected on a fritted funnel to yield $\mathbf{5}(130 \mathrm{mg}, 57 \%)$ as an off-white powder

${ }^{1} \mathbf{H}$ NMR $\left(500 \mathrm{MHz}, \mathrm{CD}_{2} \mathrm{Cl}_{2}\right) \delta 7.65(\mathrm{q}, J=7.1,5.9 \mathrm{~Hz}, 2 \mathrm{H}), 7.50-7.38(\mathrm{~m}, 2 \mathrm{H}), 7.34-7.26$ $(\mathrm{m}, 4 \mathrm{H}), 6.95(\mathrm{~d}, J=2.5 \mathrm{~Hz}, 2 \mathrm{H}), 6.92-6.86(\mathrm{~m}, 1 \mathrm{H}), 6.66(\mathrm{~d}, J=8.5 \mathrm{~Hz}, 2 \mathrm{H}), 6.59$ (br s, $1 \mathrm{H})$, 4.64 (hept, $J=6.0 \mathrm{~Hz}, 2 \mathrm{H}), 3.79$ (s, 3H), 3.67 (s, $2 \mathrm{H}), 2.35(\mathrm{~s}, 3 \mathrm{H}), 2.24-2.13(\mathrm{~m}, 2 \mathrm{H}), 1.82(\mathrm{~d}$, $J=13.5 \mathrm{~Hz}, 6 \mathrm{H}), 1.74-1.64(\mathrm{~m}, 4 \mathrm{H}), 1.64-1.54(\mathrm{~m}, 2 \mathrm{H}), 1.38(\mathrm{~d}, J=6.0 \mathrm{~Hz}, 6 \mathrm{H}), 1.33-1.17$ (m, 4H), $1.14(\mathrm{td}, J=11.8,3.7 \mathrm{~Hz}, 2 \mathrm{H}), 1.02(\mathrm{~d}, J=6.0 \mathrm{~Hz}, 6 \mathrm{H}), 0.96-0.84(\mathrm{~m}, 2 \mathrm{H})$.

${ }^{13}$ C NMR (126 MHz, $\left.\mathrm{CD}_{2} \mathrm{Cl}_{2}\right) \delta 175.2,170.0,159.7,156.1,145.2,145.1,137.8,137.7,135.5$, $133.4,133.1,133.0,132.9,131.5,131.3,131.2,131.2,130.7,128.1,126.9,126.9,115.0,111.4$, $111.3,107.6,101.2,71.5,56.0,34.3,34.1,30.4,28.7,28.5,28.5,27.5,27.4,27.2,27.1,26.5$, $22.7,22.3,22.0,21.8,21.7$.

${ }^{31} \mathbf{P}$ NMR $\left(203 \mathrm{MHz}, \mathrm{CD}_{2} \mathrm{Cl}_{2}\right) \delta 32.99$.

IR (neat): 3053, 2975, 2927, 2847, 1736, 1715, 1679, 1592, 1571, 1476, 1450, 1383, 1369, 1353, $1315,1225,1175,1135,1109,1061,1009,922,898,848,822,808,749,735,690 \mathrm{~cm}^{-1}$

M.P.: $201-204{ }^{\circ} \mathrm{C}(\mathrm{dec}$.)

HRMS (ESI) Calcd. For $\mathrm{C}_{49} \mathrm{H}_{59} \mathrm{NO}_{6} \mathrm{PPd}(\mathrm{M}-\mathrm{Cl})^{+}:$894.3109. Found: 894.3124.

(XPhos)Pd [(4-CF 3$) P h] C l(6)$

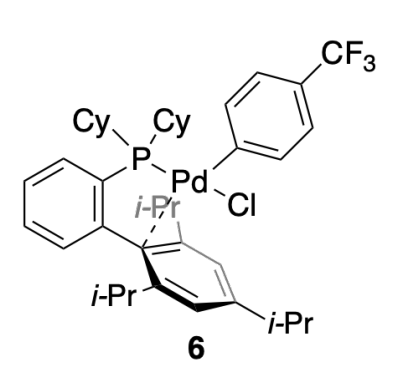

Compound 6 was prepared according to General Procedure D using S1 (178 mg, $0.250 \mathrm{mmol}, 1.0$ equiv), XPhos (131 mg, $0.275 \mathrm{mmol}$, 1.1 equiv), and $n$-hexane $(2.5 \mathrm{~mL})$. The precipitate was collected on a fritted funnel to yield $6(162 \mathrm{mg}, 85 \%)$ as a white powder.

Note: NMR spectroscopic analysis of $\mathbf{6}$ was performed at concentrations of $\sim 10 \mathrm{mg} / \mathrm{mL}$ in $\mathrm{CD}_{2} \mathrm{Cl}_{2}$. More concentrated samples lead to the formation of additional peaks in the ${ }^{1} \mathrm{H}$ NMR spectrum.

${ }^{1}$ H NMR $\left(500 \mathrm{MHz}, \mathrm{CD}_{2} \mathrm{Cl}_{2}\right) \delta 7.72-7.66(\mathrm{~m}, 1 \mathrm{H}), 7.49-7.43(\mathrm{~m}, 2 \mathrm{H}), 7.23(\mathrm{~d}, J=6.4 \mathrm{~Hz}$, $2 \mathrm{H}), 7.19$ (d, $J=8.2 \mathrm{~Hz}, 2 \mathrm{H}), 7.13(\mathrm{~s}, 2 \mathrm{H}), 6.92-6.84(\mathrm{~m}, 1 \mathrm{H}), 3.05$ (hept, $J=6.9 \mathrm{~Hz}, 1 \mathrm{H}), 2.46$ (hept, $J=6.7 \mathrm{~Hz}, 2 \mathrm{H}), 2.25-2.13(\mathrm{~m}, 2 \mathrm{H}), 1.98-1.88(\mathrm{~m}, 2 \mathrm{H}), 1.83-1.75(\mathrm{~m}, 2 \mathrm{H}), 1.76-$ $1.63(\mathrm{~m}, 6 \mathrm{H}), 1.60(\mathrm{~d}, J=6.8 \mathrm{~Hz}, 7 \mathrm{H}), 1.37(\mathrm{~d}, J=6.9 \mathrm{~Hz}, 6 \mathrm{H}), 1.31-1.05(\mathrm{~m}, 7 \mathrm{H}), 0.91(\mathrm{~d}, J=$ $6.7 \mathrm{~Hz}, 6 \mathrm{H}$ ), 0.62 (qt, $J=12.8,3.8 \mathrm{~Hz}, 2 \mathrm{H}$ ).

${ }^{13}$ C NMR $\left(126 \mathrm{MHz}, \mathrm{CD}_{2} \mathrm{Cl}_{2}\right) \delta 156.9,150.4,147.8,147.7,145.7,137.2,137.2,134.6,134.3$, $133.9,133.8,132.4,131.1,131.1,127.4,127.4,126.3,126.2,125.9,125.3,125.3,124.9,124.2$, $123.3,123.3,123.3,35.6,35.4,34.9,31.9,28.8,28.8,28.1,28.1,27.9,27.8,27.5,27.5,26.3$, 25.6, 24.8, 24.8, 24.5, 24.2 .

${ }^{19}$ F NMR $\left(471 \mathrm{MHz}, \mathrm{CD}_{2} \mathrm{Cl}_{2}\right) \delta-63.18$.

${ }^{31} \mathbf{P}$ NMR $\left(203 \mathrm{MHz}, \mathrm{CD}_{2} \mathrm{Cl}_{2}\right) \delta 29.51$.

IR (neat): 3058, 2965, 2927, 2859, 1606, 1587, 1566, 1552, 1462, 1445, 1419, 1383, 1360, 1350, $1317,1275,1208,1180,1156,1116,1095,1069,1050,1007,943,917,884,846,827,815,782$, $770,761,742,735,723,680,649,628,597 \mathrm{~cm}^{-1}$

M.P.: $230-233{ }^{\circ} \mathrm{C}(\mathrm{dec}$.)

EA Calcd. for $\mathrm{C}_{40} \mathrm{H}_{53} \mathrm{ClF}_{3} \mathrm{PPd}$ : C, 62.91; H, 7.00. Found: C, 63.21; H, 7.11. 
(XPhos)Pd[(4-CF $) \mathrm{Ph}] \mathrm{Br}(7)$

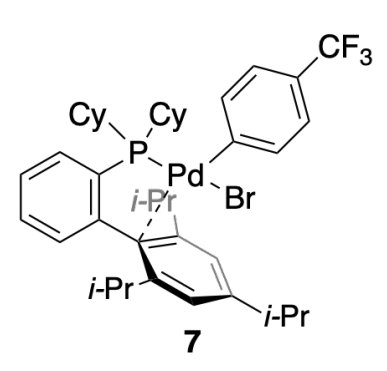

Compound 7 was prepared according to General Procedure D using S2 (189 mg, $0.250 \mathrm{mmol}, 1.0$ equiv), XPhos (131 mg, $0.275 \mathrm{mmol}$, 1.1 equiv), and $n$-hexane $(2.5 \mathrm{~mL})$. The precipitate was collected on a fritted funnel to yield $7(132 \mathrm{mg}, 65 \%)$ as a white powder.

Note: NMR spectroscopic analysis of 7 was performed at concentrations of $\sim 10 \mathrm{mg} / \mathrm{mL}$ in $\mathrm{CD}_{2} \mathrm{Cl}_{2}$. More concentrated samples lead to the formation of additional peaks on the ${ }^{1} \mathrm{H}$ NMR spectrum.

${ }^{1}$ H NMR $\left(600 \mathrm{MHz}, \mathrm{CD}_{2} \mathrm{Cl}_{2}\right) \delta 7.73-7.66(\mathrm{~m}, 1 \mathrm{H}), 7.48-7.43(\mathrm{~m}, 2 \mathrm{H}), 7.22(\mathrm{~d}, J=8.5 \mathrm{~Hz}$, 2H), $7.18(\mathrm{~d}, J=8.2 \mathrm{~Hz}, 2 \mathrm{H}), 7.14(\mathrm{~s}, 2 \mathrm{H}), 6.87-6.81(\mathrm{~m}, 1 \mathrm{H}), 3.10$ (hept, $J=6.9 \mathrm{~Hz}, 1 \mathrm{H}), 2.47$ (hept, $J=6.8 \mathrm{~Hz}, 2 \mathrm{H}), 2.26-2.16(\mathrm{~m}, 2 \mathrm{H}), 1.97-1.90(\mathrm{~m}, 2 \mathrm{H}), 1.79(\mathrm{~d}, J=11.5 \mathrm{~Hz}, 2 \mathrm{H}), 1.75$ $-1.64(\mathrm{~m}, 6 \mathrm{H}), 1.59(\mathrm{~d}, J=6.8 \mathrm{~Hz}, 7 \mathrm{H}), 1.39(\mathrm{~d}, J=6.9 \mathrm{~Hz}, 6 \mathrm{H}), 1.31-1.17(\mathrm{~m}, 5 \mathrm{H}), 1.11$ (qt, $J=12.9,3.7 \mathrm{~Hz}, 2 \mathrm{H}), 0.90(\mathrm{~d}, J=6.7 \mathrm{~Hz}, 6 \mathrm{H}), 0.59(\mathrm{qt}, J=12.9,3.8 \mathrm{~Hz}, 2 \mathrm{H})$.

${ }^{13}$ C NMR $\left(151 \mathrm{MHz}, \mathrm{CD}_{2} \mathrm{Cl}_{2}\right) \delta 156.9,150.5,147.6,147.5,143.0,137.7,137.6,134.4,134.2$, 134.0, 133.9, 132.5, 131.1, 131.1, 127.4, 127.3, 126.1, 126.0, 125.8, 125.4, 125.4, 125.2, 123.1, $123.1,123.1,35.6,35.4,34.7,31.9,28.7,28.7,28.1,28.0,27.9,27.8,27.5,27.5,26.2,25.5,24.8$, 24.6.

${ }^{19} \mathbf{F}$ NMR $\left(565 \mathrm{MHz}, \mathrm{CD}_{2} \mathrm{Cl}_{2}\right) \delta-63.14$.

${ }^{31}$ P NMR $\left(203 \mathrm{MHz}, \mathrm{CD}_{2} \mathrm{Cl}_{2}\right) \delta 27.67$.

IR (neat): $3055,2963,2930,2856,2585,1464,1443,1419,1383,1360,1317,1298,1270,1206$, $1177,1156,1118,1095,1069,1047,1009,943,915,879,846,829,818,784,768,765,742$, $732,723,680,647 \mathrm{~cm}^{-1}$

M.P.: $226-229^{\circ} \mathrm{C}(\mathrm{dec}$.)

EA Calcd. for $\mathrm{C}_{40} \mathrm{H}_{53} \mathrm{BrF}_{3} \mathrm{PPd}$ : C, 59.45; H, 6.61. Found: C, 59.66; H, 6.72.

\section{(XPhos)Pd[(4-CF 3$) P h] O T f(8)$}

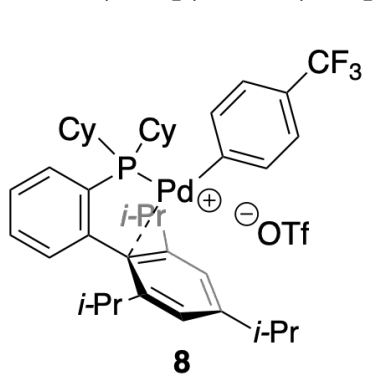

Compound $\mathbf{8}$ was prepared according to a modified General Procedure D using S3 (206 mg, $0.250 \mathrm{mmol}, 1.0$ equiv), XPhos (179 $\mathrm{mg}, 0.375 \mathrm{mmol}, 1.5$ equiv), and cyclohexane $(2.5 \mathrm{~mL})$. The reaction mixture was heated at $80^{\circ} \mathrm{C}$ for $18 \mathrm{~h}$. The precipitate was collected on a fritted funnel to yield $\mathbf{8}(204 \mathrm{mg}, 93 \%)$ as a grey powder Note: 0.5 equiv of cyclohexane precipitates with solid $\mathbf{8}$ that could not be removed even after heating at $80{ }^{\circ} \mathrm{C}$ under vacuum $(<50$ mtorr) for $24 \mathrm{~h}$.

${ }^{1} \mathbf{H}$ NMR $\left(600 \mathrm{MHz}, \mathrm{CD}_{2} \mathrm{Cl}_{2}\right) \delta 7.71(\mathrm{td}, J=7.7,1.5 \mathrm{~Hz}, 1 \mathrm{H}), 7.62-7.52(\mathrm{~m}, 2 \mathrm{H}), 7.33-7.30$ (m, 4H), 7.25 (d, $J=8.1 \mathrm{~Hz}, 2 \mathrm{H}$ ), 7.12 (ddd, $J=7.6,3.3,1.5 \mathrm{~Hz}, 1 \mathrm{H}$ ), 3.09 (hept, $J=6.9 \mathrm{~Hz}$, 1H), 2.49 (hept, $J=6.8 \mathrm{~Hz}, 2 \mathrm{H}$ ), 2.26 (tdt, $J=12.8,10.3,2.9 \mathrm{~Hz}, 2 \mathrm{H}), 1.97$ (dq, $J=10.2,3.3 \mathrm{~Hz}$, $2 \mathrm{H}), 1.84-1.77$ (m, 2H), $1.76-1.67(\mathrm{~m}, 6 \mathrm{H}), 1.65(\mathrm{~d}, J=6.8 \mathrm{~Hz}, 6 \mathrm{H}), 1.56$ (qdd, $J=12.3,5.1$, $3.6 \mathrm{~Hz}, 2 \mathrm{H}), 1.35-1.18(\mathrm{~m}, 10 \mathrm{H}), 1.10(\mathrm{ddt}, J=16.7,13.1,6.4 \mathrm{~Hz}, 2 \mathrm{H}), 0.98(\mathrm{~d}, J=6.7 \mathrm{~Hz}$, $6 \mathrm{H}), 0.62$ (qt, $J=12.8,4.1 \mathrm{~Hz}, 2 \mathrm{H})$.

${ }^{13}$ C NMR $\left(151 \mathrm{MHz}, \mathrm{CD}_{2} \mathrm{Cl}_{2}\right) \delta 155.9,150.9,146.2,146.1,144.0,137.5,137.5,136.7,134.7$, 134.6, 133.6, 133.3, 133.2, 133.1, 132.5, 132.0, 131.9, 128.3, 128.2, 128.2, 128.1, 127.9, 127.6, 
$125.8,124.0,124.0,124.0,123.9,123.7,123.6,123.1,122.3,121.3,119.1,36.3,36.1,34.9,34.5$, 34.1, 32.1, 31.7, 28.9, 28.1, 28.1, 27.6, 27.5, 27.2, 26.3, 26.1, 26.0, 24.1, 23.9, 23.4, 22.7, 22.3.

${ }^{19}$ F NMR $\left(377 \mathrm{MHz}, \mathrm{CDCl}_{3}\right) \delta-63.59,-79.99$.

${ }^{31}$ P NMR $\left(162 \mathrm{MHz}, \mathrm{CDCl}_{3}\right) \delta 37.05$.

IR (neat): 2953, 2930, 2859, 1601, 1585, 1561, 1485, 1466, 1450, 1426, 1386, 1362, 1324, 1307 , $1270,1260,1225,1201,1173,1151,1135,1116,1097,1069,1052,1028,1012,1007,960,941$, $922,893,881,846,818,775,758,747,735,720,678,666,630 \mathrm{~cm}^{-1}$

M.P.: $172-174{ }^{\circ} \mathrm{C}($ dec.)

EA Calcd. for $\mathrm{C}_{88} \mathrm{H}_{118} \mathrm{~F}_{12} \mathrm{O}_{6} \mathrm{P}_{2} \mathrm{Pd}_{2} \mathrm{~S}_{2}\left\{(\mathrm{XPhos}) \mathrm{Pd}\left[\left(4-\mathrm{CF}_{3}\right) \mathrm{Ph}\right] \mathrm{OTf}\right\}{ }_{2} \mathrm{Cy}$ : C, 57.48; H, 6.47. Found: C, 57.45; H, 6.54.

HRMS (ESI) Calcd. For $\mathrm{C}_{40} \mathrm{H}_{53} \mathrm{~F}_{3} \mathrm{PPd}(\mathrm{M}-\mathrm{OTf})^{+}$: 727.2866. Found: 727.2883.

(XPhos)Pd[(4-CF 3$) P h] O M s ~(9)$

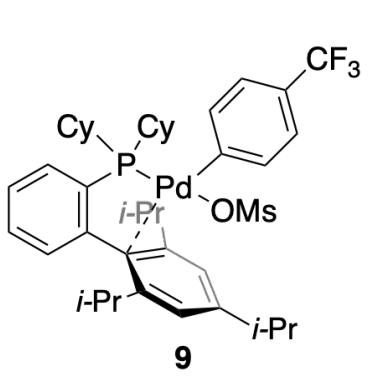

Compound 9 was prepared according to General Procedure D using S4 (193 mg, $0.250 \mathrm{mmol}, 1.0$ equiv), XPhos (131 mg, $0.275 \mathrm{mmol}$, 1.1 equiv), and $n$-hexane $(2.5 \mathrm{~mL})$. The precipitate was collected on a fritted funnel to yield 9 (193 $\mathrm{mg}, 94 \%)$ as a white powder

${ }^{1} \mathbf{H}$ NMR $\left(500 \mathrm{MHz}, \mathrm{CD}_{2} \mathrm{Cl}_{2}\right) \delta 7.69(\mathrm{t}, J=7.9 \mathrm{~Hz}, 1 \mathrm{H}), 7.53-7.46$ $(\mathrm{m}, 2 \mathrm{H}), 7.44(\mathrm{~d}, J=5.6 \mathrm{~Hz}, 2 \mathrm{H}), 7.27(\mathrm{~s}, 1 \mathrm{H}), 7.26(\mathrm{~s}, 3 \mathrm{H}), 6.95(\mathrm{dt}$, $J=6.3,2.9 \mathrm{~Hz}, 1 \mathrm{H}), 3.21$ (hept, $J=6.9 \mathrm{~Hz}, 1 \mathrm{H}$ ), 2.47 (hept, $J=6.7$ $\mathrm{Hz}, 2 \mathrm{H}), 2.29-2.17(\mathrm{~m}, 2 \mathrm{H}), 2.08-2.01(\mathrm{~m}, 2 \mathrm{H}), 1.84-1.77(\mathrm{~m}$, 2H), $1.69(\mathrm{~d}, J=6.8 \mathrm{~Hz}, 12 \mathrm{H}), 1.59$ (dt, $J=12.1,4.2 \mathrm{~Hz}, 2 \mathrm{H}), 1.45$ (s, 3H), 1.32 (d, $J=6.9 \mathrm{~Hz}$, $7 \mathrm{H}), 1.27-1.16(\mathrm{~m}, 3 \mathrm{H}), 1.16-1.03(\mathrm{~m}, 2 \mathrm{H}), 0.93(\mathrm{~d}, J=6.6 \mathrm{~Hz}, 6 \mathrm{H}), 0.59$ (q, $J=12.6 \mathrm{~Hz}$, $2 \mathrm{H})$.

${ }^{13}$ C NMR $\left(126 \mathrm{MHz}, \mathrm{CD}_{2} \mathrm{Cl}_{2}\right) \delta 157.2,150.8,147.6,147.4,145.8,139.1,139.1,137.8,137.8$, 133.6, 133.5, 133.3, 133.0, 132.1, 131.4, 131.4, 128.2, 127.9, 127.6, 127.6, 127.5, 127.4, 127.1, $126.1,124.7,123.9,123.8,123.8,123.6,123.6,123.6,121.8,39.4,35.9,35.6,33.7,32.1,31.3$, $31.3,28.9,28.0,28.0,27.8,27.7,27.5,27.4,26.2,26.2,26.0,24.3,23.3$.

${ }^{19}$ F NMR $\left(471 \mathrm{MHz}, \mathrm{CD}_{2} \mathrm{Cl}_{2}\right) \delta-63.40$.

${ }^{31} \mathbf{P}$ NMR $\left(203 \mathrm{MHz}, \mathrm{CD}_{2} \mathrm{Cl}_{2}\right) \delta 35.61$.

IR (neat): 3053, 2951, 2920, 2861, 1604, 1585, 1559, 1485, 1462, 1445, 1426, 1386, 1362, 1331 , $1260,1246,1211,1185,1128,1118,1097,1071,1052,1012,1007,953,924,893,881,874$, $853,815,770,761,756,744,735,725,680,647 \mathrm{~cm}^{-1}$

M.P.: $195-197^{\circ} \mathrm{C}($ dec.)

EA Calcd. for $\mathrm{C}_{41} \mathrm{H}_{56} \mathrm{~F}_{3} \mathrm{O}_{3} \mathrm{PPdS}$ : C, 59.81; H, 6.86. Found: C, 59.57; H, 6.96.

(RuPhos)Pd[(4-CF $)$ Ph]Cl (10)

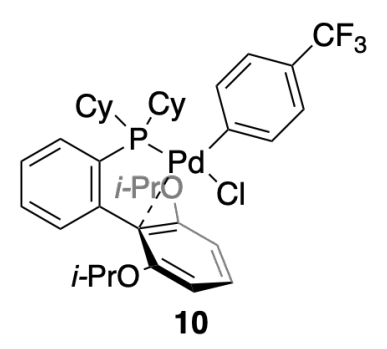

Compound 10 was prepared according to General Procedure D using S1 (178 mg, $0.250 \mathrm{mmol}, 1.0$ equiv), RuPhos (128 mg, $0.275 \mathrm{mmol}$, 1.1 equiv), and $n$-hexane $(2.5 \mathrm{~mL})$. The precipitate was collected on a fritted funnel to yield $9(167 \mathrm{mg}, 89 \%)$ as a white powder

${ }^{1}$ H NMR $\left(600 \mathrm{MHz}, \mathrm{CD}_{2} \mathrm{Cl}_{2}\right) \delta 7.66(\mathrm{t}, J=8.4 \mathrm{~Hz}, 1 \mathrm{H}), 7.63-7.59$ $(\mathrm{m}, 1 \mathrm{H}), 7.46(\mathrm{tt}, J=7.5,1.5 \mathrm{~Hz}, 1 \mathrm{H}), 7.40(\mathrm{tt}, J=7.5,1.4 \mathrm{~Hz}, 1 \mathrm{H})$, $7.28(\mathrm{~d}, J=6.3 \mathrm{~Hz}, 2 \mathrm{H}), 7.18(\mathrm{~d}, J=8.1 \mathrm{~Hz}, 2 \mathrm{H}), 6.88(\mathrm{ddd}, J=7.8$, 
3.1, $1.4 \mathrm{~Hz}, 1 \mathrm{H}), 6.66(\mathrm{~d}, J=8.5 \mathrm{~Hz}, 2 \mathrm{H}), 4.64$ (hept, $J=6.0 \mathrm{~Hz}, 2 \mathrm{H}), 2.11$ (tdt, $J=12.4,9.7,2.7$ $\mathrm{Hz}, 2 \mathrm{H}), 1.77$ (br d, $J=6.5 \mathrm{~Hz}, 6 \mathrm{H}), 1.66$ (br d, $J=11.2 \mathrm{~Hz}, 4 \mathrm{H}$ ), 1.57 (tdd, $J=12.4,9.1,5.2$ $\mathrm{Hz}, 2 \mathrm{H}), 1.39(\mathrm{~d}, J=6.0 \mathrm{~Hz}, 6 \mathrm{H}), 1.26-1.15(\mathrm{~m}, 4 \mathrm{H}), 1.15-1.05(\mathrm{~m}, 2 \mathrm{H}), 1.02(\mathrm{~d}, J=6.1 \mathrm{~Hz}$, $6 \mathrm{H}), 0.73(\mathrm{qt}, J=12.9,3.9 \mathrm{~Hz}, 2 \mathrm{H})$.

${ }^{13}$ C NMR $\left(151 \mathrm{MHz}, \mathrm{CD}_{2} \mathrm{Cl}_{2}\right) \delta 159.7,146.9,145.2,145.1,137.6,137.6,135.4,133.4,133.2$, $132.9,132.8,131.4,131.1,131.1,126.9,126.8,126.2,126.1,125.9,125.7,125.5,124.4,123.0$, 123.0, 111.2, 111.2, 107.6, 71.4, 34.1, 33.9, 28.6, 28.1, 28.1, 27.5, 27.4, 27.1, 27.1, 26.4, 22.3, 21.7.

${ }^{19}$ F NMR $\left(565 \mathrm{MHz}, \mathrm{CD}_{2} \mathrm{Cl}_{2}\right) \delta-63.14$.

${ }^{31}$ P NMR (202 MHz, $\left.\mathrm{CD}_{2} \mathrm{Cl}_{2}\right) \delta 33.28$.

IR (neat): 3095, 3062, 2965, 2937, 2918, 2849, 1585, 1455, 1381, 1367, 1346, 1320, 1293, 1270, 1258, 1244, 1204, 1151, 1132, 1109, 1095, 1069, 1057, 1007, 948, 919, 900, 886, 848, 846, 815, $777,768,761,742,735,720,680,668,616,597 \mathrm{~cm}^{-1}$

M.P.: $209-211^{\circ} \mathrm{C}(\mathrm{dec}$.

HRMS (ESI) Calcd. For $\mathrm{C}_{37} \mathrm{H}_{47} \mathrm{~F}_{3} \mathrm{O}_{2} \mathrm{PPd}(\mathrm{M}-\mathrm{Cl})^{+}$: 717.2295. Found: 717.2310.

\section{(BrettPhos)Pd[(4-CF $) \mathrm{Ph}] \mathrm{Cl}(11)$}

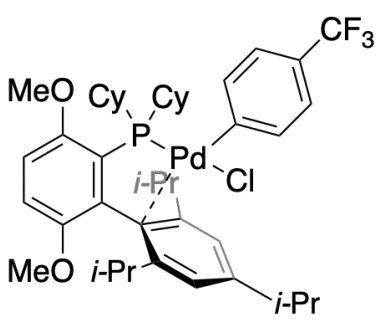

11 C-bound

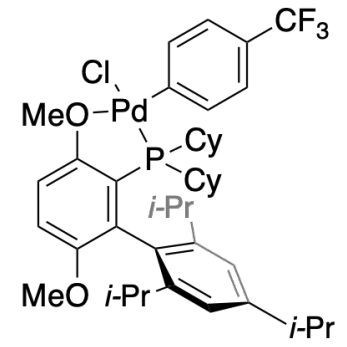

11 O-bound

Compound 11 was prepared according to a modified General Procedure D using S1 (178 mg, $0.250 \mathrm{mmol}, 1.0$ equiv), BrettPhos (148 mg, $0.275 \mathrm{mmol}, 1.1$ equiv), and 1,4-dioxane ( $2.5 \mathrm{~mL})$. The reaction mixture was heated at $80{ }^{\circ} \mathrm{C}$ for $1 \mathrm{~h}$. The precipitate was collected on a fritted funnel to yield $11(174 \mathrm{mg}, 85 \%)$ as a pale-yellow powder Note: Compound 11 was determined to be an 8:1 mixture of $C$-Bound to $O$-Bound isomer in solution.

${ }^{1} \mathbf{H}$ NMR $\left(600 \mathrm{MHz}, \mathrm{CD}_{2} \mathrm{Cl}_{2}\right)$ Complex spectrum, see below.

${ }^{13} \mathbf{C}$ NMR $\left(151 \mathrm{MHz}, \mathrm{CD}_{2} \mathrm{Cl}_{2}\right) \delta 157.6,156.9,156.8,155.2,155.2,154.5,154.4,153.0,152.7$, $152.4,152.3,149.6,149.3,147.3,144.6,138.9,138.9,138.5,138.5,138.5,138.3,137.8,137.8$, $135.6,133.5,133.5,130.8,130.7,128.1,126.3,126.2,125.7,125.6,125.5,125.4,125.3,125.1$, $125.0,124.8,124.5,122.7,122.6,122.6,122.0,120.7,118.3,118.1,117.0,117.0,114.1,114.1$, 111.7, 111.6, 111.5, 111.4, 67.4, 61.2, 55.3, 55.2, 54.8, 36.6, 36.5, 35.6, 35.4, 34.9, 34.7, 31.8, $31.3,29.8,29.7,28.3,28.1,28.0,28.0,27.0,26.9,26.7,26.7,26.5,25.6,25.5,24.8,24.6,24.1$, 23.5.

${ }^{19} \mathbf{F}$ NMR $\left(565 \mathrm{MHz}, \mathrm{CD}_{2} \mathrm{Cl}_{2}\right) \delta-63.02,-63.22$.

${ }^{31}$ P NMR $\left(202 \mathrm{MHz}, \mathrm{CD}_{2} \mathrm{Cl}_{2}\right) \delta 47.87,38.69$.

IR (neat): 3058, 2958, 2925, 2854, 2833, 1606, 1582, 1549, 1483, 1459, 1447, 1424, 1381, 1360 , $1317,1279,1260,1241,1213,1175,1149,1111,1092,1083,1066,1007,941,917,889,872$, $851,829,822,813,794,768,754,739,720,711,675,649,619 \mathrm{~cm}^{-1}$ 
M.P.: $241-242{ }^{\circ} \mathrm{C}($ dec.)

EA Calcd. for $\mathrm{C}_{42} \mathrm{H}_{57} \mathrm{ClF}_{3} \mathrm{O}_{2} \mathrm{PPd}$ : C, 61.24; H, 6.97. Found: C, 60.98; H, 7.02.

\section{(XPhos)Pd(Ph)Cl (12)}

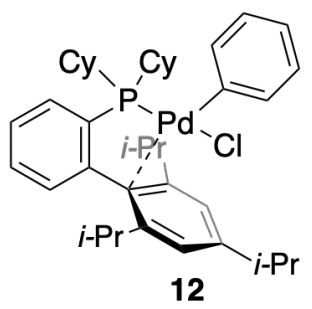

Compound 12 was prepared according to a modified General Procedure D using ( $t$-BuXPhos) $\mathrm{Pd}(\mathrm{Ph}) \mathrm{Cl}(161 \mathrm{mg}, 0.250 \mathrm{mmol}, 1.0$ equiv), XPhos (131 mg, $0.275 \mathrm{mmol}, 1.1$ equiv), and 2-MeTHF (2.5 $\mathrm{mL})$. Once the reaction mixture had cooled to room temperature, the reaction tube was opened to air and the reaction mixture was transferred to a $20 \mathrm{~mL}$ scintillation vial with the assistance of pentane $(5.0 \mathrm{~mL})$. The combined solution was concentrated in vacuo with the aid of a rotary evaporator to yield a viscous oil. The oil was layered with pentane $(5.0 \mathrm{~mL})$ and triturated with the assistance of sonication to form a homogenous solution. The vial was gently scratched with a glass stir rod, then capped and placed in a freezer at $-25{ }^{\circ} \mathrm{C}$ for $\sim 22 \mathrm{~h}$. Over this period, a grey crystalline precipitate formed. The mixture was triturated with the assistance of sonication for $\sim 1 \mathrm{~min}$ and returned to the $-25^{\circ} \mathrm{C}$ freezer for $1 \mathrm{~h}$. Over this period, a grey precipitate had formed. The precipitate was collected on a fritted funnel and washed with cold pentane $\left(\sim-25^{\circ} \mathrm{C}, 25 \mathrm{~mL}\right)$ and dried in vacuo on the Schlenk line overnight $(\sim 16 \mathrm{~h})$ to yield $12(128 \mathrm{mg}, 74 \%)$ as a grey powder.

Note: NMR spectroscopic analysis of 12 was performed at concentrations of $\sim 10 \mathrm{mg} / \mathrm{mL}$ in $\mathrm{CD}_{2} \mathrm{Cl}_{2}$. When more concentrated samples are prepared, additional peaks in the ${ }^{1} \mathrm{H}$ NMR spectrum are observed.

${ }^{1} \mathbf{H}$ NMR $\left(600 \mathrm{MHz}, \mathrm{CD}_{2} \mathrm{Cl}_{2}\right) \delta 7.73-7.67(\mathrm{~m}, 1 \mathrm{H}), 7.47-7.41(\mathrm{~m}, 2 \mathrm{H}), 7.12(\mathrm{~s}, 2 \mathrm{H}), 7.06-$ $7.01(\mathrm{~m}, 2 \mathrm{H}), 6.93(\mathrm{t}, J=7.3 \mathrm{~Hz}, 2 \mathrm{H}), 6.90-6.84(\mathrm{~m}, 2 \mathrm{H}), 3.05$ (hept, $J=6.9 \mathrm{~Hz}, 1 \mathrm{H}), 2.46$ (hept, $J=6.8 \mathrm{~Hz}, 2 \mathrm{H}), 2.21(\mathrm{dtd}, J=12.5,9.9,2.9 \mathrm{~Hz}, 2 \mathrm{H}), 1.92(\mathrm{t}, J=6.6 \mathrm{~Hz}, 2 \mathrm{H}), 1.82-1.74$ $(\mathrm{m}, 2 \mathrm{H}), 1.73-1.62(\mathrm{~m}, 8 \mathrm{H}), 1.59(\mathrm{~d}, J=6.8 \mathrm{~Hz}, 6 \mathrm{H}), 1.37(\mathrm{~d}, J=6.9 \mathrm{~Hz}, 6 \mathrm{H}), 1.28-1.06(\mathrm{~m}$, $6 \mathrm{H}), 0.90(\mathrm{~d}, J=6.7 \mathrm{~Hz}, 6 \mathrm{H}), 0.62(\mathrm{qt}, J=12.9,3.8 \mathrm{~Hz}, 2 \mathrm{H})$.

${ }^{13}$ C NMR $\left(151 \mathrm{MHz}, \mathrm{CD}_{2} \mathrm{Cl}_{2}\right) \delta 156.1,149.8,148.0,147.8,138.1,138.1,137.0,137.0,134.9$, $134.7,133.8,133.7,132.4,130.8,130.8,127.5,127.2$, 127.2, 126.2, 126.2, 124.6, 123.7, 35.5, $35.3,34.8,31.8,28.7,28.7,28.0,28.0,28.0,27.9,27.6,27.5,26.3,25.6,24.7,24.5$.

${ }^{31} \mathbf{P}$ NMR $\left(203 \mathrm{MHz}, \mathrm{CD}_{2} \mathrm{Cl}_{2}\right) \delta 28.72$.

IR (neat): 3053, 2958, 2920, 2852, 1606, 1559, 1457, 1440, 1414, 1381, 1362, 1315, 1267, 1237 , 1199, 1175, 1151, 1104, 1078, 1054, 1016, 995, 957, 936, 912, 874, 846, 815, 782, 763, 730, $711,694,654 \mathrm{~cm}^{-1}$

M.P.: $211-213^{\circ} \mathrm{C}($ dec.)

HRMS (ESI) Calcd. For $\mathrm{C}_{39} \mathrm{H}_{54} \mathrm{PPd}(\mathrm{M}-\mathrm{Cl})^{+}$: 659.2992 . Found: 659.3006 . 


\section{(XPhos)Pd[(4-OMe)Ph]Cl (13)}

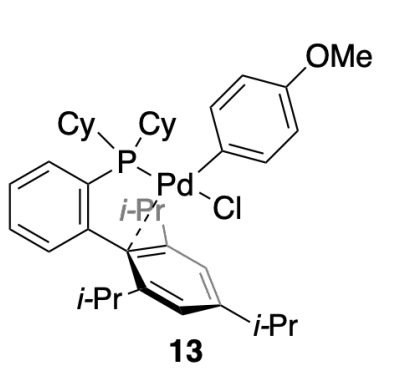

Compound 13 was prepared according to a modified General Procedure D using S6 (168 mg, $0.250 \mathrm{mmol}, 1.0$ equiv), XPhos (131 $\mathrm{mg}, 0.275 \mathrm{mmol}, 1.1$ equiv), and 2-MeTHF $(2.5 \mathrm{~mL})$. Once the reaction mixture had cooled to room temperature, the reaction tube was opened to air and the reaction mixture was transferred to a 20 $\mathrm{mL}$ scintillation vial with the assistance of pentane $(5.0 \mathrm{~mL})$. The combined solution was concentrated in vacuo with the aid of a rotary evaporator to yield a viscous oil. The oil was layered with pentane $(5.0 \mathrm{~mL})$ and triturated with the assistance of sonication to form a homogenous solution. The vial was gently scratched with a glass stir rod, then capped and placed in a freezer at $-25{ }^{\circ} \mathrm{C}$ for $\sim 22 \mathrm{~h}$. Over this period, a grey crystalline precipitate formed. The mixture was triturated with the assistance of sonication for $\sim 1 \mathrm{~min}$ and returned to the $-25{ }^{\circ} \mathrm{C}$ freezer for $1 \mathrm{~h}$. Over this period, a grey precipitate had formed. The precipitate was collected on a fritted funnel and washed with cold pentane $\left(\sim-25^{\circ} \mathrm{C}, 25 \mathrm{~mL}\right)$ and dried in vacuo on the Schlenk line overnight $(\sim 16 \mathrm{~h})$ to yield $\mathbf{1 3}(129 \mathrm{mg}, 71 \%)$ as a grey powder.

Note: NMR spectroscopic analysis of $\mathbf{1 3}$ was performed at concentrations of $\sim 10 \mathrm{mg} / \mathrm{mL}$ in $\mathrm{CD}_{2} \mathrm{Cl}_{2}$. When more concentrated samples are prepared, additional peaks in the ${ }^{1} \mathrm{H}$ NMR spectrum are observed.

${ }^{1}$ H NMR $\left(600 \mathrm{MHz}, \mathrm{CD}_{2} \mathrm{Cl}_{2}\right) \delta 7.70(\mathrm{td}, J=6.3,3.1 \mathrm{~Hz}, 1 \mathrm{H}), 7.44(\mathrm{ddd}, J=7.4,3.8,2.0 \mathrm{~Hz}$, 2H), $7.11(\mathrm{~s}, 2 \mathrm{H}), 6.87$ (app dt, $J=9.1,2.6 \mathrm{~Hz}, 3 \mathrm{H}), 6.61(\mathrm{~d}, J=8.5 \mathrm{~Hz}, 2 \mathrm{H}), 3.70(\mathrm{~s}, 3 \mathrm{H}), 3.04$ (hept, $J=7.0 \mathrm{~Hz}, 1 \mathrm{H}$ ), 2.46 (hept, $J=6.8 \mathrm{~Hz}, 2 \mathrm{H}$ ), 2.21 (dtd, $J=12.5,9.8,2.8 \mathrm{~Hz}, 2 \mathrm{H}$ ), $1.98-$ $1.90(\mathrm{~m}, 2 \mathrm{H}), 1.74-1.61(\mathrm{~m}, 7 \mathrm{H}), 1.59(\mathrm{~d}, J=6.8 \mathrm{~Hz}, 6 \mathrm{H}), 1.37(\mathrm{~d}, J=6.9 \mathrm{~Hz}, 6 \mathrm{H}), 1.28-1.07$ $(\mathrm{m}, 7 \mathrm{H}), 0.90(\mathrm{~d}, J=6.7 \mathrm{~Hz}, 6 \mathrm{H}), 0.68(\mathrm{qt}, J=12.8,3.8 \mathrm{~Hz}, 2 \mathrm{H})$.

${ }^{13}$ C NMR $\left(126 \mathrm{MHz}, \mathrm{CD}_{2} \mathrm{Cl}_{2}\right) \delta 157.4,156.1,149.8,148.0,147.9,136.6,136.6,135.2,134.9$, $133.8,133.7,132.4,130.8,130.8,127.2,127.2,126.2,126.2,125.3,125.2,124.6,113.8,113.8$, $55.5,35.7,35.5,34.8,31.8,28.8,28.8,28.2,28.1,28.0,27.9,27.7,27.6,26.3,26.3,25.6,24.7$, 24.5, 22.7 .

${ }^{31} \mathbf{P}$ NMR $\left(203 \mathrm{MHz}, \mathrm{CD}_{2} \mathrm{Cl}_{2}\right) \delta 28.98$.

IR (neat): 3050, 2958, 2923, 2852, 1606, 1578, 1563, 1481, 1459, 1445, 1419, 1381, 1360, 1317, 1298, 1265, 1230, 1173, 1116, 1097, 1069, 1052, 1028, 1002, 936, 912, 872, 853, 808, 794, 780, $765,735,713,649,585 \mathrm{~cm}^{-1}$

M.P.: $167-170{ }^{\circ} \mathrm{C}(\mathrm{dec}$.

HRMS (ESI) Calcd. For $\mathrm{C}_{40} \mathrm{H}_{56} \mathrm{OPPd}(\mathrm{M}-\mathrm{Cl})^{+}$: 689.3098. Found: 689.3111.

\section{(RuPhos)Pd[(2-Me-4-CF 3$) P h] B r(14)$}

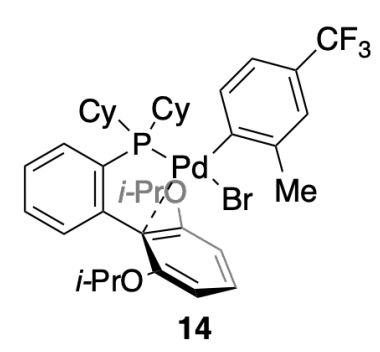

Compound $\mathbf{1 4}$ was prepared according to a modified General Procedure D using S5 (193 mg, $0.250 \mathrm{mmol}, 1.0$ equiv), XPhos (131 $\mathrm{mg}, 0.275 \mathrm{mmol}, 1.1$ equiv), and $n$-hexane $(2.5 \mathrm{~mL})$. The reaction mixture was heated at $60^{\circ} \mathrm{C}$ for $2 \mathrm{~h}$. The precipitate was collected on a fritted funnel to yield $\mathbf{1 4}(133 \mathrm{mg}, 66 \%)$ as a white powder Note: Compound $\mathbf{1 4}$ is a 1:1 mixture of atropisomers

${ }^{1}$ H NMR $\left(600 \mathrm{MHz}, \mathrm{CD}_{2} \mathrm{Cl}_{2}\right) \delta 7.68(\mathrm{t}, J=8.4 \mathrm{~Hz}, 1 \mathrm{H}), 7.61-7.57$ (m, 1H), $7.44(\mathrm{tt}, J=7.6,1.6 \mathrm{~Hz}, 1 \mathrm{H}), 7.38(\mathrm{t}, J=7.5 \mathrm{~Hz}, 1 \mathrm{H}), 7.08$ 
$-7.00(\mathrm{~m}, 3 \mathrm{H}), 6.81(\mathrm{dd}, J=7.7,1.8 \mathrm{~Hz}, 1 \mathrm{H}), 6.62(\mathrm{~d}, J=8.4 \mathrm{~Hz}, 1 \mathrm{H}), 6.58(\mathrm{~d}, J=8.4 \mathrm{~Hz}, 1 \mathrm{H})$, 4.57 (dhept, $J=24.2,6.2 \mathrm{~Hz}, 2 \mathrm{H}), 2.67(\mathrm{~s}, 3 \mathrm{H}), 2.50(\mathrm{t}, J=11.1 \mathrm{~Hz}, 1 \mathrm{H}), 2.28-2.19(\mathrm{~m}, 1 \mathrm{H})$, $2.13(\mathrm{ddd}, J=9.1,6.2,3.1 \mathrm{~Hz}, 1 \mathrm{H}), 1.82(\mathrm{q}, J=14.9,13.7 \mathrm{~Hz}, 3 \mathrm{H}), 1.77-1.67(\mathrm{~m}, 2 \mathrm{H}), 1.67-$ $1.56(\mathrm{~m}, 3 \mathrm{H}), 1.52-1.38(\mathrm{~m}, 3 \mathrm{H}), 1.35(\mathrm{dd}, J=15.1,6.0 \mathrm{~Hz}, 6 \mathrm{H}), 1.31-1.21(\mathrm{~m}, 1 \mathrm{H}), 1.20-$ $1.00(\mathrm{~m}, 9 \mathrm{H}), 0.89(\mathrm{~d}, J=6.1 \mathrm{~Hz}, 3 \mathrm{H}), 0.28(\mathrm{qd}, J=12.5,4.8 \mathrm{~Hz}, 1 \mathrm{H})$.

${ }^{13}$ C NMR $\left(151 \mathrm{MHz}, \mathrm{CD}_{2} \mathrm{Cl}_{2}\right) \delta 161.2,160.6,145.6,145.5,145.1,144.4,144.4,136.2,136.1$, $136.1,135.1,134.8,134.5,132.6,132.6,131.4,131.2,131.2,131.2,128.2,126.8,126.8,126.4$, 126.2, 126.0, 125.8, 125.6, 124.6, 123.6, 123.6, 123.6, 123.6, 122.8, 120.8, 120.8, 120.8, 120.7, $109.8,109.8,107.3,107.2,72.2,70.7,35.2,35.0,32.8,32.7,30.7,30.7,28.9,28.9,28.9,27.6$, 27.6, 27.5, 27.5, 27.2, 27.1, 26.9, 26.8, 26.6, 26.3, 26.3, 25.6, 22.7, 22.1, 21.9, 21.8.

${ }^{19}$ F NMR $\left(565 \mathrm{MHz}, \mathrm{CD}_{2} \mathrm{Cl}_{2}\right) \delta-62.94$.

${ }^{31} \mathbf{P}$ NMR $\left(203 \mathrm{MHz}, \mathrm{CD}_{2} \mathrm{Cl}_{2}\right) \delta 31.63$.

IR (neat): 2975, 2930, 2852, 1585, 1568, 1450, 1424, 1393, 1383, 1372, 1324, 1279, 1267, 1249, $1189,1159,1109,1076,1061,1021,1002,941,917,898,881,846,815,765,747,737,716,671$ $\mathrm{cm}^{-1}$

M.P.: $220-224^{\circ} \mathrm{C}$ (dec.)

EA Calcd. for $\mathrm{C}_{38} \mathrm{H}_{49} \mathrm{BrF}_{3} \mathrm{O}_{2} \mathrm{PPd}$ : C, 56.20; H, 6.08. Found: C, 56.20; H, 6.11. 


\section{Pd-Mediated Cross-Coupling Reactions}

Table 1
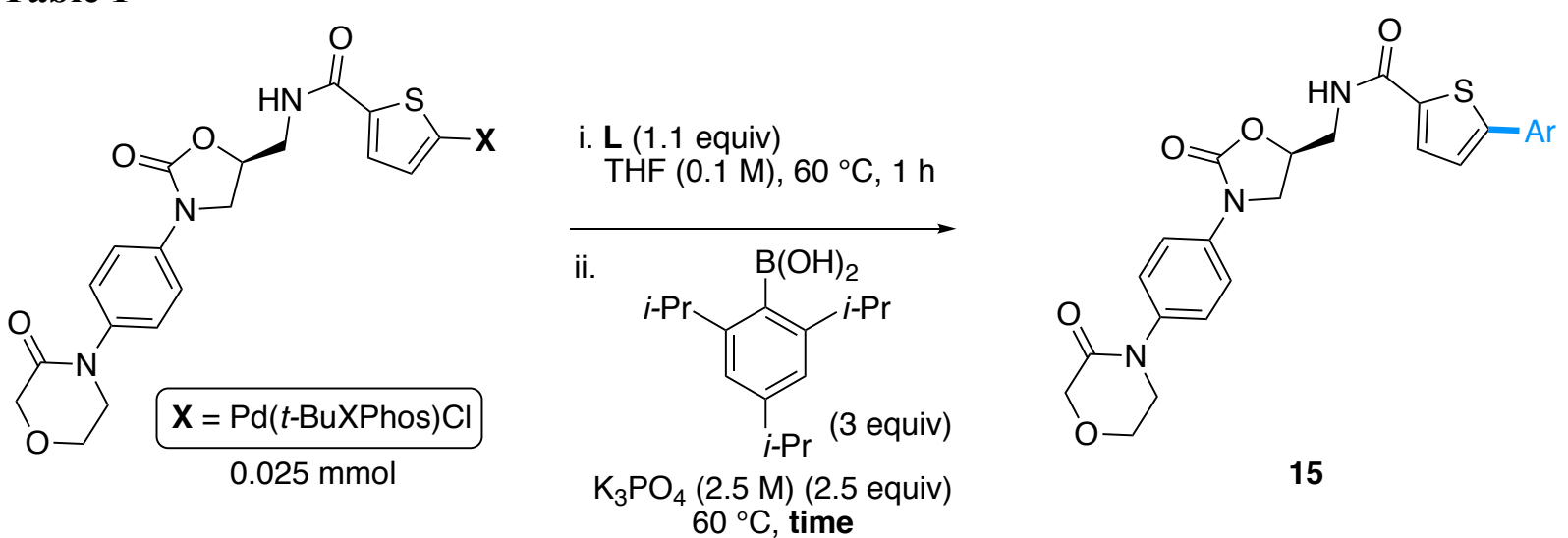

\begin{tabular}{ccccc}
\hline Entry & L & Time $(\mathrm{h})$ & Conversion (\%) & Yield 15 (\%) \\
\hline 1 & None & 1 & 100 & 0 \\
2 & SPhos & 2 & 50 & 23 \\
3 & SPhos & 16 & 90 & 36 \\
4 & CPhos & 2 & 92 & 24 \\
5 & RuPhos & 2 & 95 & 21 \\
6 & XPhos & 2 & 91 & 40 \\
7 & CPhos & 16 & 100 & 28 \\
8 & RuPhos & 16 & 100 & 21 \\
\hline 9 & XPhos & 16 & 100 & 43 \\
\hline
\end{tabular}

An oven-dried $8 \mathrm{~mL}$ reaction tube was equipped, while still hot, with a magnetic stir bar and septum cap. The tube was allowed to cool to room temperature under vacuum by piercing the septum with a needle connected to a dual manifold by a rubber hose. At this point, the cap was removed and the tube was charged with ( $t$-BuXPhos)Pd(rivaroxaban) $\mathrm{Cl}$ ( $24 \mathrm{mg}, 0.025 \mathrm{mmol}, 1.0$ equiv) and $\mathrm{L}(0.028 \mathrm{mmol}, 1.1$ equiv). The tube was recapped, the septum was pierced with a needle connected to a dual manifold by a rubber hose, and the tube was evacuated and backfilled with nitrogen (the evacuation/backfill process was repeated a total of three times). Under nitrogen, THF $(250 \mu \mathrm{L})$ was added via syringe. The tube was then placed in a preheated oil bath (bath temperature $=60^{\circ} \mathrm{C}$ ). The reaction mixture was allowed to stir at $60^{\circ} \mathrm{C}$ for $1 \mathrm{~h}$. After $1 \mathrm{~h}$, the reaction vessel was removed from the oil bath and the reaction mixture was allowed to cool for $5 \mathrm{~min}$. At this time, the cap was removed and the flask was charged with 2,4,6-tri-isopropylphenylboronic acid (19 $\mathrm{mg}, 0.150 \mathrm{mmol}, 3.0$ equiv). The cap was returned and aqueous $\mathrm{K}_{3} \mathrm{PO}_{4}$ (degassed, $2.5 \mathrm{M}, 25 \mu \mathrm{L}, 2.5$ equiv) was added via syringe. The needle connecting the tube to the dual manifold was removed and the cap was sealed with parafilm. The tube was then placed in a preheated oil bath (bath temperature $=60{ }^{\circ} \mathrm{C}$ ). The reaction mixture was allowed to stir at $60{ }^{\circ} \mathrm{C}$ for $16 \mathrm{~h}$. After $16 \mathrm{~h}$, the reaction vessel was removed from the oil bath and the reaction mixture was allowed to cool to room temperature. The reaction tube was opened to air and the reaction mixture was charged with a caffeine solution $\left(100 \mu \mathrm{L}, 0.25 \mathrm{M}\right.$ in $\mathrm{CH}_{2} \mathrm{Cl}_{2}, 1.0$ 
equiv). $10 \mu \mathrm{L}$ of the resulting solution was removed and transferred to a $2 \mathrm{~mL} \mathrm{LC}$ vial containing $1.5 \mathrm{~mL}$ DMSO. LC yield was determined by UV peak area $(254 \mathrm{~nm})$ of $\mathbf{1 5}$ against caffeine as an internal standard. ${ }^{2}$

Conversion was determined by UV peak area $(254 \mathrm{~nm})$ on the LC/MS using the formula:

$$
\% \text { Conversion } \text { PV }_{\text {roduct }}=\left(\frac{\text { Area }_{\text {Product }}}{\sum \text { Area }_{\text {Product }}+\text { Area }_{\text {Reduction }}+\text { Area }_{\mathrm{OAC}}}\right)
$$

(S)-N-((2-oxo-3-(4-(3-oxomorpholino)phenyl)oxazolidin-5-yl)methyl)-5-(2,4,6triisopropylphenyl)thiophene-2-carboxamide (15)<smiles>CCCc1cc(C(C)C)cc(C(C)C)c1-c1ccc(C(=O)NCC2CN(c3ccc(N4CCOCC4=O)cc3)C(=O)O2)s1</smiles>

Via OAC ligand exchange: An oven-dried $8 \mathrm{~mL}$ reaction tube was equipped, while still hot, with a magnetic stir bar and septum cap. The tube was allowed to cool to room temperature under vacuum by piercing the septum with a needle connected to a dual manifold by a rubber hose. At this point, the cap was removed and the tube was charged with ( $t$ BuXPhos)Pd(rivaroxaban)Cl (48 mg, $0.050 \mathrm{mmol}, 1.0$ equiv) and XPhos (26 mg, $0.055 \mathrm{mmol}$, 1.1 equiv). The tube was recapped, the septum was pierced with a needle connected to a dual manifold by a rubber hose, and the tube was evacuated and backfilled with nitrogen (the evacuation/backfill process was repeated a total of three times). Under nitrogen, THF $(500 \mu \mathrm{L})$ was added via syringe. The tube was then placed in a preheated oil bath (bath temperature $=60$ ${ }^{\circ} \mathrm{C}$ ). The reaction mixture was allowed to stir at $60^{\circ} \mathrm{C}$ for $1 \mathrm{~h}$. After $1 \mathrm{~h}$, the reaction vessel was removed from the oil bath and the reaction mixture was allowed to cool for $5 \mathrm{~min}$. At this time, the cap was removed and the flask was charged with 2,4,6-tri-iso-propylphenylboronic acid (37 $\mathrm{mg}, 0.150 \mathrm{mmol}, 3.0$ equiv). The cap was returned and aqueous $\mathrm{K}_{3} \mathrm{PO}_{4}$ (degassed, $2.5 \mathrm{M}, 50 \mu \mathrm{L}$, 2.5 equiv) was added via syringe. The needle connecting the tube to the dual manifold was removed and the cap was sealed with parafilm. The tube was then placed in a preheated oil bath (bath temperature $=60{ }^{\circ} \mathrm{C}$ ). The reaction mixture was allowed to stir at $60{ }^{\circ} \mathrm{C}$ for $16 \mathrm{~h}$. After 16 $\mathrm{h}$, the reaction vessel was removed from the oil bath and the reaction mixture was allowed to cool to room temperature. The cap was then removed and DMT resin (200 mg) and $\mathrm{CH}_{2} \mathrm{Cl}_{2}(500$ $\mu \mathrm{L})$ were added. The tube was recapped and the reaction mixture was allowed to stir for $1 \mathrm{~h}$ at room temperature. After $1 \mathrm{~h}$, the cap was removed and the reaction mixture was filtered through $\mathrm{a} \sim 1 \mathrm{~cm}$ plug of Celite ${ }^{\circledR}$ into a $100 \mathrm{~mL}$ round-bottom flask. The Celite ${ }^{\circledR}$ plug was further washed with $\mathrm{CH}_{2} \mathrm{Cl}_{2}(\sim 25 \mathrm{~mL})$ and $\mathrm{MeOH}(\sim 25 \mathrm{~mL})$. The combined filtrate solution was concentrated in vacuo with the assistance of a rotary evaporator. The resulting residue was purified by preparatory thin layer chromatography (97.5:2.5 EtOAc: $\mathrm{MeOH}$, eluted twice) to yield $\mathbf{1 5}$ (13 mg, $43 \%$ ) as a white powder.

Via a Stoichiometric amount of XPhos Pd G4: An oven-dried $8 \mathrm{~mL}$ reaction tube was equipped, while still hot, with a magnetic stir bar and septum cap. The tube was allowed to cool to room temperature under vacuum by piercing the septum with a needle connected to a dual 
manifold by a rubber hose. At this point, the cap was removed and the tube was charged with rivaroxaban (22 $\mathrm{mg}, 0.050 \mathrm{mmol}, 1.0$ equiv), XPhos Pd G4 (43 mg, $0.050 \mathrm{mmol}, 1.0$ equiv) and 2,4,6-tri-iso-propylphenylboronic acid ( $37 \mathrm{mg}, 0.150 \mathrm{mmol}, 3.0$ equiv). The tube was recapped, the septum was pierced with a needle connected to a dual manifold by a rubber hose, and the tube was evacuated and backfilled with nitrogen (the evacuation/backfill process was repeated a total of three times). Under nitrogen, THF $(500 \mu \mathrm{L})$ was added via syringe, followed by aqueous $\mathrm{K}_{3} \mathrm{PO}_{4}$ (degassed, $2.5 \mathrm{M}, 70 \mu \mathrm{L}, 3.5$ equiv). The needle connecting the tube to the dual manifold was removed and the cap was sealed with parafilm. The tube was then placed in a preheated oil bath (bath temperature $=60^{\circ} \mathrm{C}$ ). The reaction mixture was allowed to stir at $60^{\circ} \mathrm{C}$ for $16 \mathrm{~h}$. At this time, the reaction vessel was removed from the oil bath and the reaction mixture was allowed to cool to room temperature. The cap was then removed and DMT resin (200 $\mathrm{mg})$ and $\mathrm{CH}_{2} \mathrm{Cl}_{2}(500 \mu \mathrm{L})$ were added. The cap was returned and the reaction mixture was allowed to stir for $1 \mathrm{~h}$ at room temperature. The cap was removed and the reaction mixture was filtered through $\mathrm{a} \sim 1 \mathrm{~cm}$ plug of Celite ${ }^{\circledR}$ into a $100 \mathrm{~mL}$ round-bottom flask. The Celite ${ }^{\circledR}$ plug was further washed with $\mathrm{CH}_{2} \mathrm{Cl}_{2}(\sim 25 \mathrm{~mL})$ and $\mathrm{MeOH}(\sim 25 \mathrm{~mL})$. The combined filtrate solution was concentrated in vacuo with the assistance of a rotary evaporator. The resulting residue was purified by preparatory thin layer chromatography (97.5:2.5 EtOAc: $\mathrm{MeOH}$, eluted twice) to yield 15 (11 mg, $37 \%$ ) as a white powder.

By using 10 mol\% XPhos Pd G4 (4 mg, 0.005 mmol, 0.1 equiv), 15 was obtained (10 mg, 33\%) as a white powder.

${ }^{1} \mathbf{H}$ NMR $\left(600 \mathrm{MHz}, \mathrm{CD}_{2} \mathrm{Cl}_{2}\right) \delta 7.58(\mathrm{~d}, J=8.9 \mathrm{~Hz}, 2 \mathrm{H}), 7.55-7.52(\mathrm{~m}, 1 \mathrm{H}), 7.35(\mathrm{~d}, J=9.0$ $\mathrm{Hz}, 2 \mathrm{H}), 7.07(\mathrm{~s}, 2 \mathrm{H}), 6.83(\mathrm{~d}, J=3.7 \mathrm{~Hz}, 1 \mathrm{H}), 6.69(\mathrm{br} \mathrm{s}, 1 \mathrm{H}), 4.90-4.83(\mathrm{~m}, 1 \mathrm{H}), 4.28(\mathrm{~s}, 2 \mathrm{H})$, $4.12(\mathrm{t}, J=8.9 \mathrm{~Hz}, 1 \mathrm{H}), 4.03-3.98(\mathrm{~m}, 2 \mathrm{H}), 3.86(\mathrm{dd}, J=9.1,6.7 \mathrm{~Hz}, 2 \mathrm{H}), 3.76-3.70(\mathrm{~m}, 3 \mathrm{H})$, 2.92 (hept, $J=6.9 \mathrm{~Hz}, 1 \mathrm{H}), 2.74$ (hept, $J=6.9 \mathrm{~Hz}, 2 \mathrm{H}), 1.27$ (d, $J=6.9 \mathrm{~Hz}, 6 \mathrm{H}), 1.14$ (dd, $J=$ $6.9,2.7 \mathrm{~Hz}, 6 \mathrm{H}), 1.11(\mathrm{dd}, J=6.9,4.6 \mathrm{~Hz}, 6 \mathrm{H})$.

${ }^{13}$ C NMR $\left(151 \mathrm{MHz}, \mathrm{CD}_{2} \mathrm{Cl}_{2}\right) \delta 167.0,162.8,154.7,150.5,149.1,147.6,138.1,137.9,137.2$, $128.9,128.8,127.8,126.6,121.2,119.2,72.4,68.9,64.5,50.0,48.1,42.8,34.8,31.0,24.7,24.4$, 24.2.

IR (neat): 3460, 3275, 2956, 2927, 2871, 1758, 1672, 1658, 1637, 1606, 1542, 1521, 1469, 1457, $1438,1428,1414,1381,1357,1341,1329,1310,1289,1260,1227,1159,1140,1121,1095$, $1071,1050,1016,995,960,943,919,877,860,836,822,815,796,775,747.735,709,687$, $666,614 \mathrm{~cm}^{-1}$

M.P.: $265-270{ }^{\circ} \mathrm{C}$

HRMS (ESI) Calcd. For $\mathrm{C}_{34} \mathrm{H}_{42} \mathrm{~N}_{3} \mathrm{O}_{5} \mathrm{~S}(\mathrm{M}+\mathrm{H})^{+}$: 604.2840. Found: 604.2849. 


\section{Pd-Catalyzed Cross-Coupling Reactions}

\section{Scheme 1}

A. ${ }^{a}$
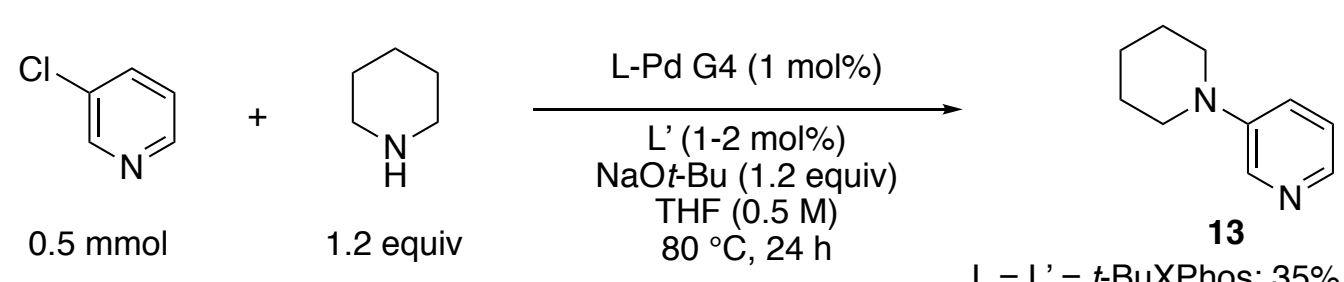

$\mathrm{L}=\mathrm{L}^{\prime}=t$-BuXPhos: $35 \%$

$L=L^{\prime}=$ RuPhos: $85 \%$

$L=t$-BuXPhos; L' = RuPhos: $85 \%$

B. ${ }^{b}$<smiles>[O]O</smiles>

t-BuXPhos-Pd G4 (1 mol\%)

RuPhos (2 mol\%)

$\mathrm{NaO} t$-Bu (1.2 equiv)

THF $(0.5 \mathrm{M})$

$80^{\circ} \mathrm{C}, 24 \mathrm{~h}$<smiles>c1cncc(N2CCCCC2)c1</smiles>

$13,78 \%$

Scheme 1A: An oven-dried $8 \mathrm{~mL}$ reaction tube was equipped, while still hot, with a magnetic stir bar and septum cap. The tube was allowed to cool to room temperature under vacuum by piercing the septum with a needle connected to a dual manifold by a rubber hose. At this point, the cap was removed and the tube was charged with L-Pd G4 (0.005 mmol, $1 \mathrm{~mol} \%), \mathrm{L}$ ' (0.005 mmol, $1 \mathrm{~mol} \%$ ), $\mathrm{NaOt}$-Bu (58 mg, $0.600 \mathrm{mmol}, 1.2$ equiv) and 1,3,5-trimethoxybenzene (42 $\mathrm{mg}$, $0.250 \mathrm{mmol}, 0.5$ equiv). The reaction tube was brought into a nitrogen-filled glovebox and charged with 3 -chloropyridine ( $48 \mu \mathrm{L}, 0.500 \mathrm{mmol}, 1.0$ equiv), piperidine ( $59 \mu \mathrm{L}, 0.600 \mathrm{mmol}$, 1.2 equiv), and THF (1.0 mL) via micropipettor. The reaction tube was capped with an unpunctured septum cap and removed from the glovebox. The cap was covered with parafilm and the tube was placed in a preheated oil bath (bath temperature $=80{ }^{\circ} \mathrm{C}$ ). The reaction mixture was allowed to stir for $24 \mathrm{~h}$ at $80{ }^{\circ} \mathrm{C}$. At this time, the reaction vessel was removed from the oil bath and the reaction mixture was allowed to cool to room. The cap was removed and the reaction mixture was filtered through a $\sim 2 \mathrm{~cm}$ Celite ${ }^{\circledR}$ plug in a Pasteur pipette. The Celite ${ }^{\circledR}$ plug was further washed with $\mathrm{CH}_{2} \mathrm{Cl}_{2}(5 \mathrm{~mL})$. The combined filtrate solution was concentrated in vacuo with the assistance of a rotary evaporator. The resulting residue was taken up in $\mathrm{CDCl}_{3}$ (1 $\mathrm{mL}$ ) and the yield was determined by quantitative ${ }^{1} \mathrm{H}$ NMR spectroscopy against $1,3,5-$ trimethoxybenzene as an internal standard.

\section{3-(piperidin-1-yl)pyridine (16)}

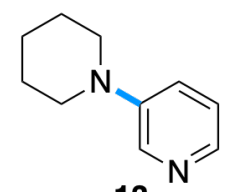

Scheme 1B: An oven-dried $16 \mathrm{~mL}$ reaction tube was equipped, while still hot, with a magnetic stir bar and septum cap. The tube was allowed to cool to room temperature under vacuum by piercing the septum with a needle connected to a dual manifold by a rubber hose. At this point, the cap was removed and the tube was charged with $t$-BuXPhos Pd G4 (8 mg, $0.01 \mathrm{mmol}$, $1 \mathrm{~mol} \%$ ), RuPhos (9 mg, $0.02 \mathrm{mmol}, 2 \mathrm{~mol} \%$ ), and $\mathrm{NaOt}$-Bu (115 mg, 1.20 mmol, 1.2 equiv). The reaction tube was brought into a nitrogen-filled glovebox and charged with 3-chloropyridine ( $95 \mu \mathrm{L}, 1.00 \mathrm{mmol}, 1.0$ equiv), piperidine ( $119 \mu \mathrm{L}, 1.20 \mathrm{mmol}, 1.2$ equiv), and THF $(2.0 \mathrm{~mL})$ via micropipettor. The reaction tube was capped with an unpunctured septum cap and removed from the glovebox. The cap was covered with parafilm and the tube was placed 
in a preheated oil bath (bath temperature $=80{ }^{\circ} \mathrm{C}$ ). The reaction mixture was allowed to stir for $24 \mathrm{~h}$ at $80^{\circ} \mathrm{C}$. At this point, the reaction vessel was removed from the oil bath and the reaction mixture was allowed to cool to room. The cap was removed and the reaction mixture was filtered through a $\sim 1 \mathrm{~cm}$ Celite ${ }^{\circledR}$ plug in a $15 \mathrm{~mL}$ fritted funnel. The Celite ${ }^{\circledR}$ plug was further washed with $\mathrm{CH}_{2} \mathrm{Cl}_{2}(25 \mathrm{~mL})$. The combined filtrate solution was concentrated in vacuo with the assistance of a rotary evaporator. The crude material was purified by column chromatography (40 $\mathrm{g} \mathrm{SiO}_{2}$, EtOAc, $16 \times 100 \mathrm{~mm}$ fractions, UV) to yield $16(126 \mathrm{mg}, 78 \%$ ) as a yellow-colored oil.

${ }^{1} \mathbf{H}$ NMR $\left(500 \mathrm{MHz}, \mathrm{CDCl}_{3}\right) \delta 8.31(\mathrm{~d}, J=2.9 \mathrm{~Hz}, 1 \mathrm{H}), 8.05(\mathrm{dd}, J=4.4,1.4 \mathrm{~Hz}, 1 \mathrm{H}), 7.17$ $(\mathrm{ddd}, J=8.5,3.0,1.5 \mathrm{~Hz}, 1 \mathrm{H}), 7.12(\mathrm{dd}, J=8.4,4.5 \mathrm{~Hz}, 1 \mathrm{H}), 3.21-3.15(\mathrm{~m}, 4 \mathrm{H}), 1.71(\mathrm{p}, J=$ $5.7 \mathrm{~Hz}, 4 \mathrm{H}), 1.60$ (qd, $J=8.2,7.1,4.4 \mathrm{~Hz}, 2 \mathrm{H})$.

${ }^{13}$ C NMR (126 MHz, $\left.\mathrm{CDCl}_{3}\right) \delta 147.9,140.3,139.2,123.5,122.7,50.0,25.7,24.2$. 


\section{References}

1. Fulmer, G. R.; Miller, A. J. M.; Sherden, M. H.; Gottlieb, H. E.; Nudelman, A.; Stoltz, B. M.; Bercaw, J. E.; Goldberg, K. I. NMR chemical shifts of trace impurities: common laboratory solvents, organics, and gases in deuterated solvents relevant to the organometallic chemist. Organometallics 2010, 29, 2176-2179.

2. Uehling, M. R.; King, R. P.; Krska, S. W.; Cernak, T.; Buchwald, S. L. Pharmaceutical diversification via palladium oxidative addition complexes. Science 2019, 363, 405-408.

3. Lee, H. G.; Milner, P. J.; Colvin, M. T.; Andreas, L.; Buchwald, S. L. Structure and reactivity of [(L·Pd $)_{n} \cdot(1,5$-cyclooctadiene $\left.)\right](n=1-2)$ complexes bearing biaryl phosphine ligands. Inorg. Chim. Acta. 2014, 422, 188-192.

4. Lovinger, G. J.; Aparece, M. D.; Morken, J. P. Pd-Catalyzed Conjunctive Cross-Coupling between Grignard-Derived Boron "Ate" Complexes and $\mathrm{C}\left(\mathrm{sp}^{2}\right)$ Halides or Triflates: NaOTf as a Grignard Activator and Halide Scavenger. J. Am. Chem. Soc. 2017, 139, 3153-3160.

5. Han, C.; Buchwald, S. L. Negishi Coupling of Secondary Alkylzinc Halides with Aryl Bromides and Chlorides. J. Am. Chem. Soc. 2009, 131, 7532-7533.

6. Barder, T. E.; Walker, S. D.; Martinelli, J. R.; Buchwald, S. L. Catalysts for Suzuki-Miyaura Coupling Processes: Scope and Studies of the Effect of Ligand Structure. J. Am. Chem. Soc. 2005, 127, 4685-4696.

7. Bruno, N. C.; Niljianskul, N.; Buchwald, S. L. N-Substituted 2-Aminobiphenylpalladium Methanesulfonate Precatalysts and Their Use in $\mathrm{C}-\mathrm{C}$ and $\mathrm{C}-\mathrm{N}$ Cross-Couplings. J. Org. Chem. 2014, 79, 4161-4166. 


\section{Copies of NMR Spectra}




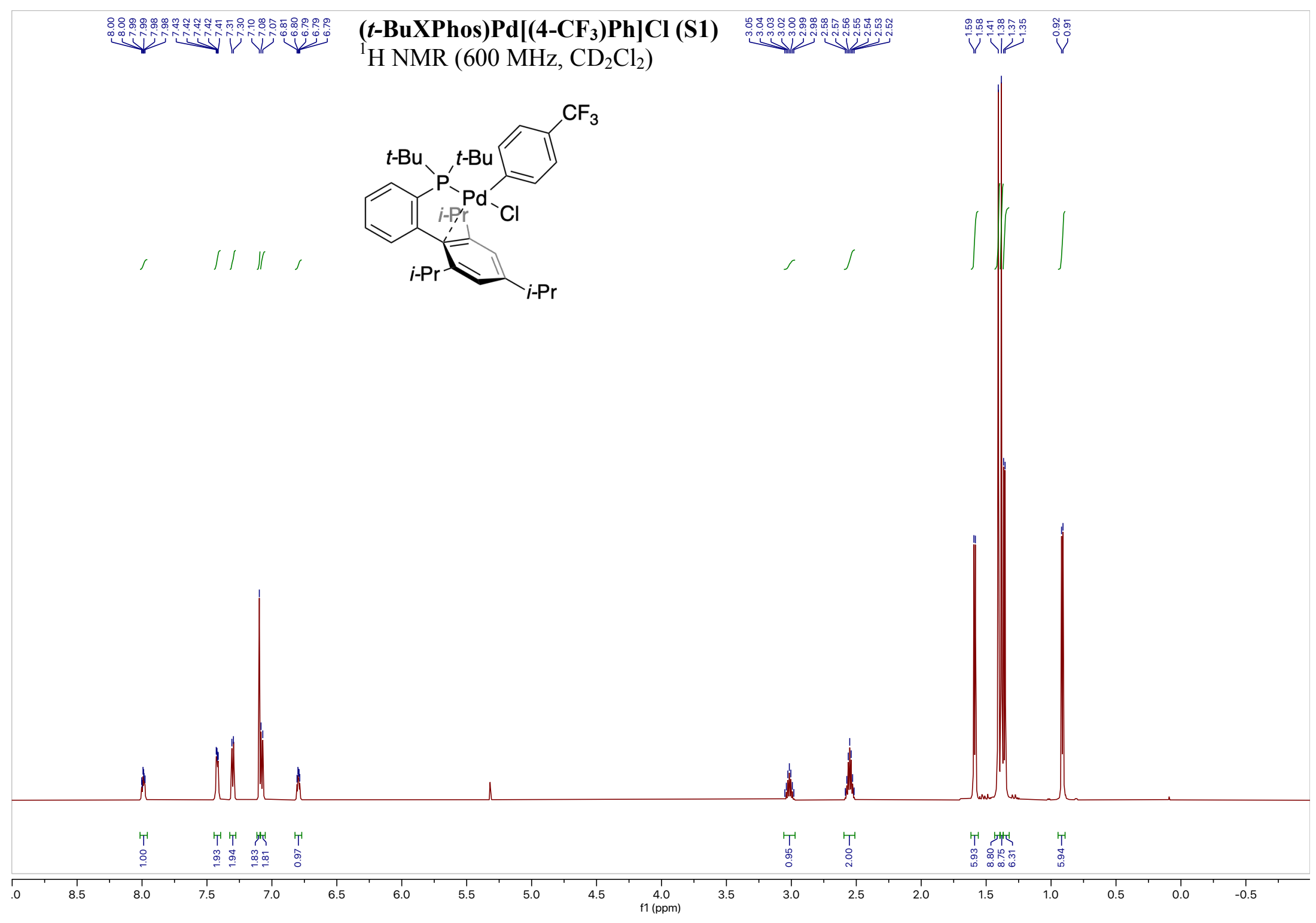




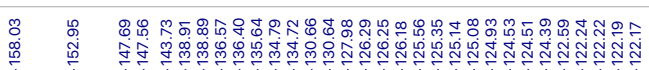

(t-BuXPhos)Pd[(4-CF 3 )Ph]Cl (S1)

${ }^{13} \mathrm{C}$ NMR (151 MHz, $\mathrm{CD}_{2} \mathrm{Cl}_{2}$ )
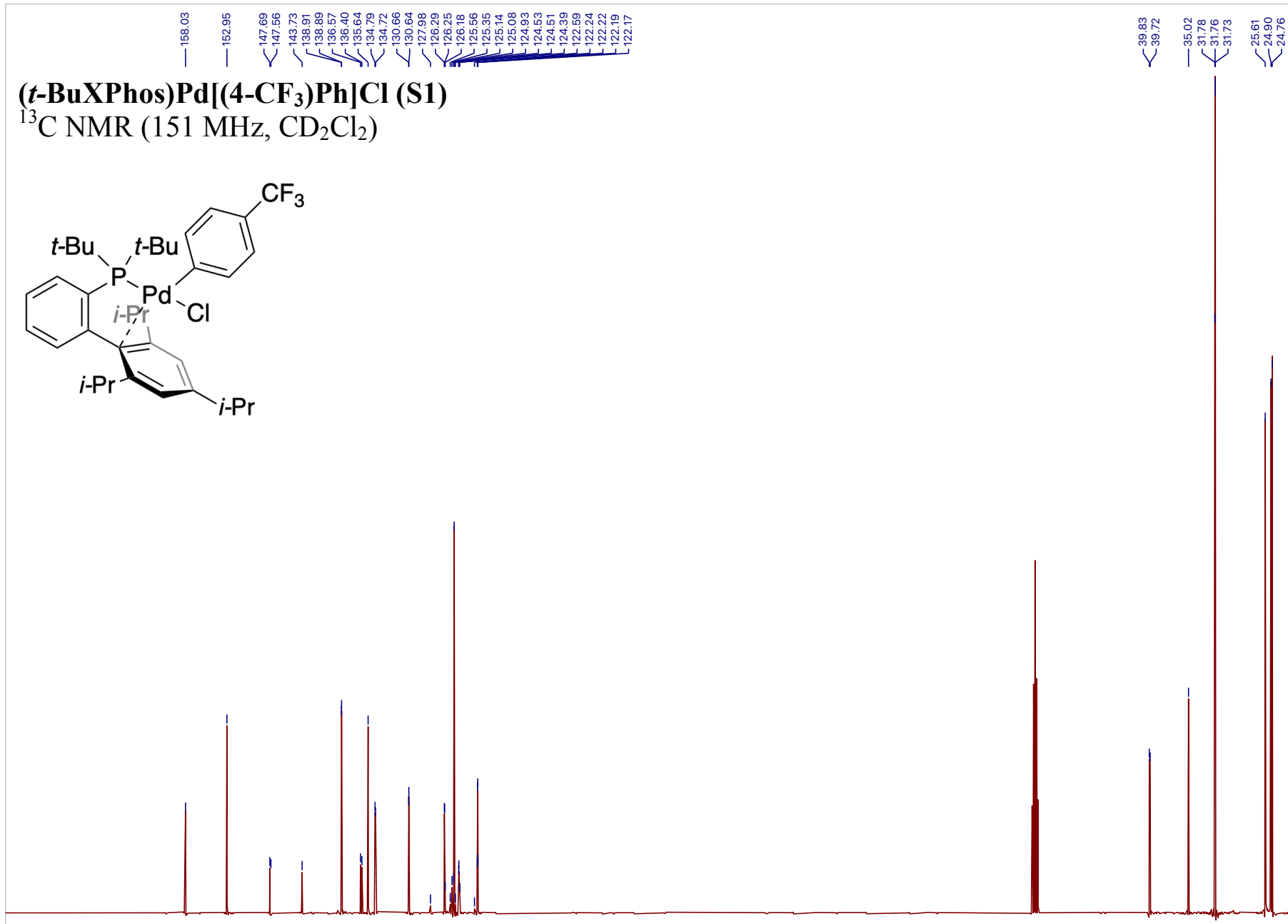

170 160 150 140 130 120 110 100 $90 \underset{f 1(\mathrm{ppm})}{80}$ $70+60$ 50 40 30 20

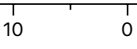


(t-BuXPhos)Pd[(4-CF 3$) \mathrm{Ph}] \mathrm{Cl}(\mathrm{S} 1)$

${ }^{19} \mathrm{~F}$ NMR $\left(565 \mathrm{MHz}, \mathrm{CD}_{2} \mathrm{Cl}_{2}\right.$ )

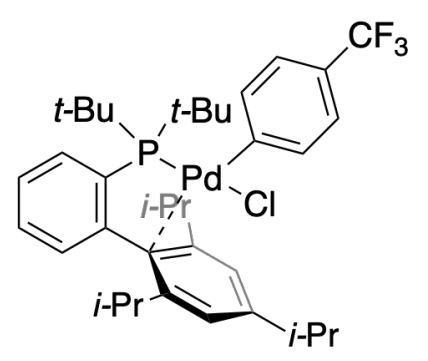


(t-BuXPhos)Pd[(4-CF 3$) P h] C l$ (S1)

${ }^{31} \mathrm{P}$ NMR (202 MHz, $\mathrm{CD}_{2} \mathrm{Cl}_{2}$ )

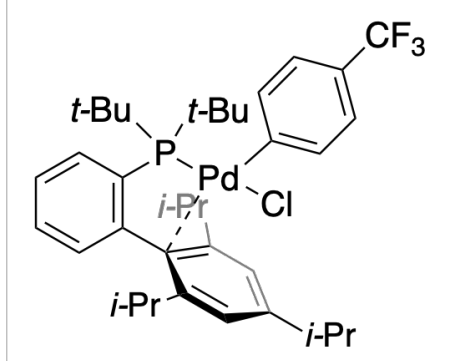

50

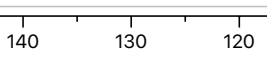
110 100 1
90
90 60 50
$\mathrm{f} 1(\mathrm{ppm})$ 


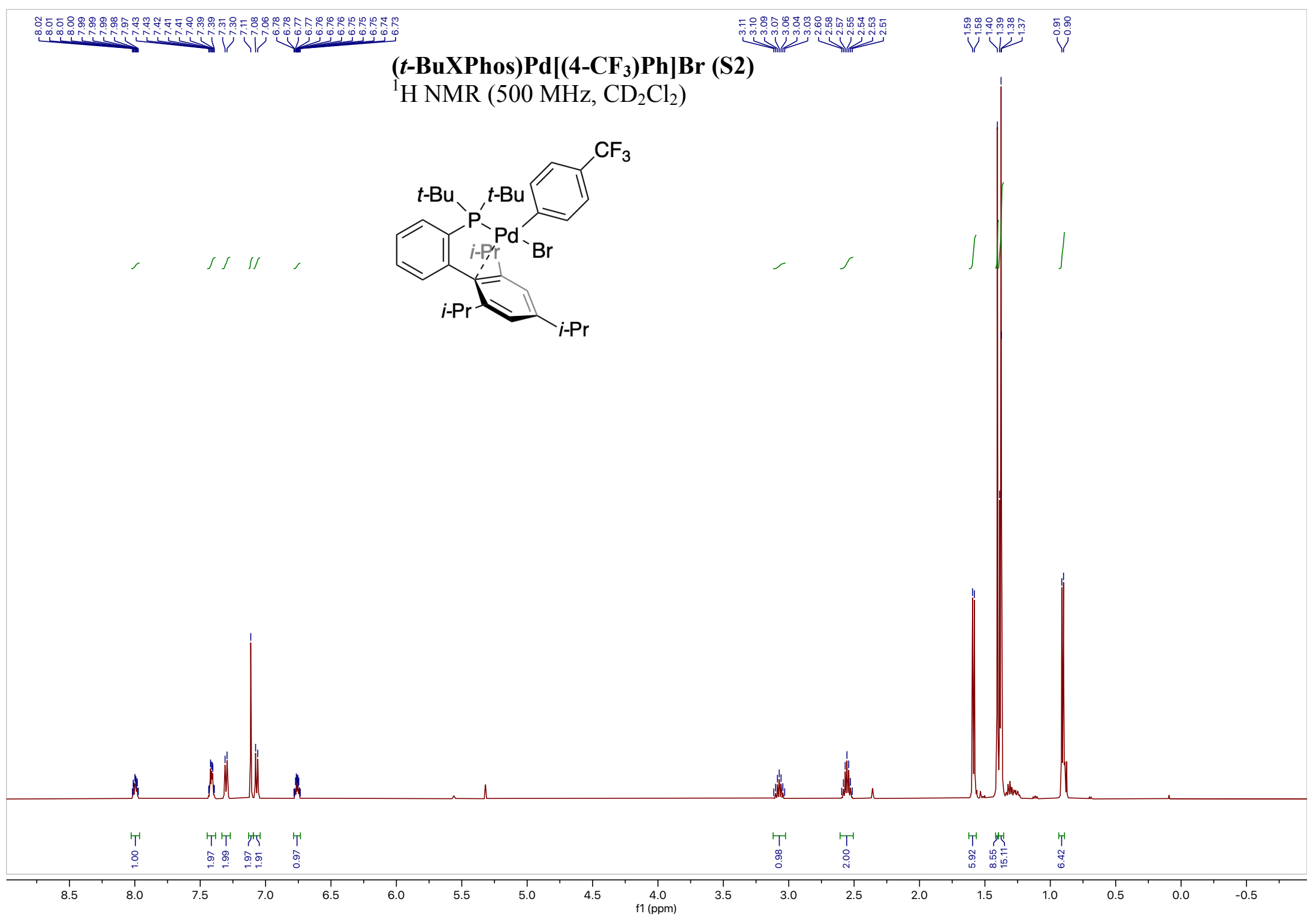




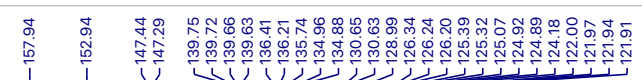

$1 \sqrt{2}$

(t-BuXPhos)Pd[(4-CF 3 )Ph]Br (S2)

${ }^{13} \mathrm{C}$ NMR (126 MHz, $\mathrm{CD}_{2} \mathrm{Cl}_{2}$ )
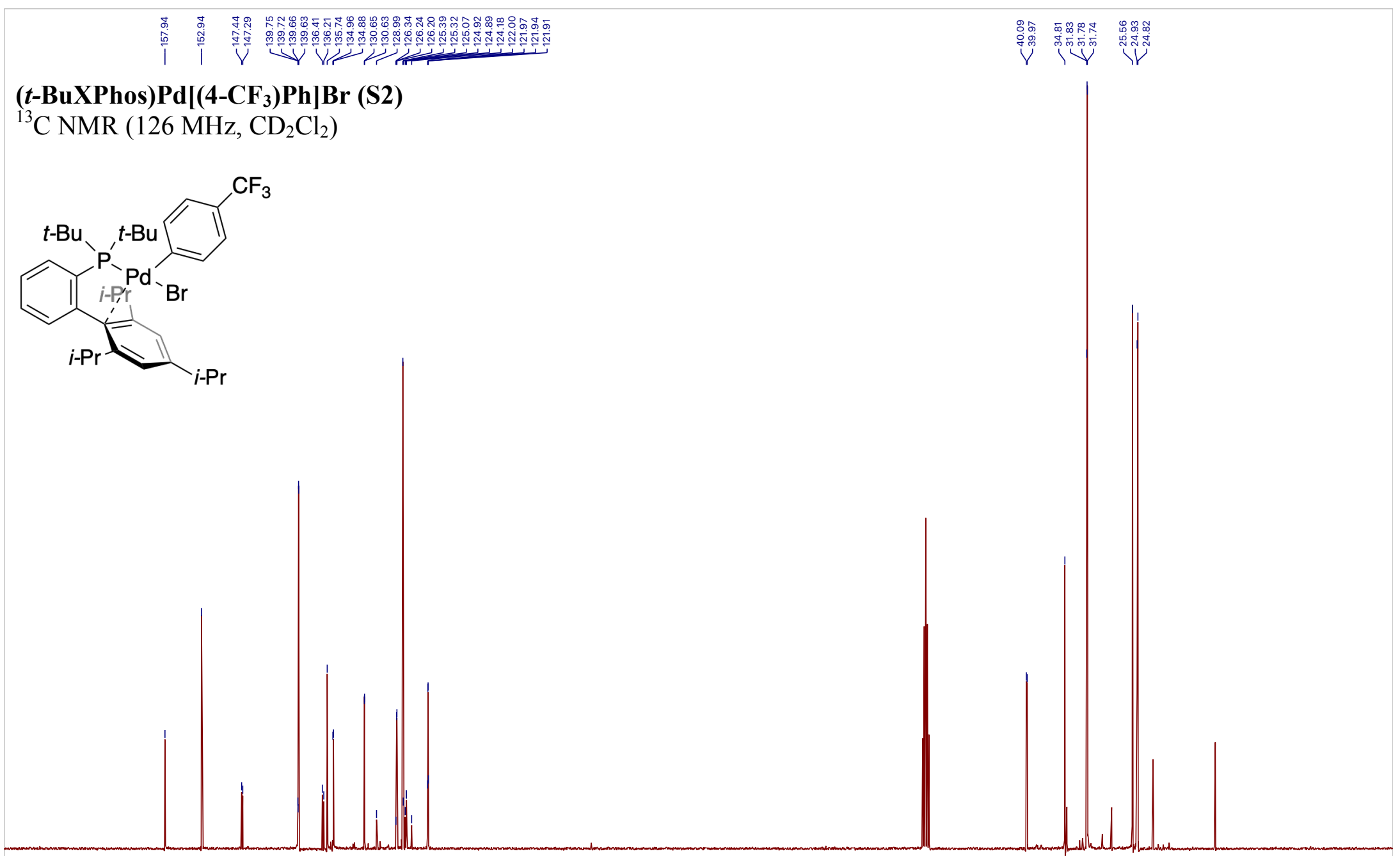

170

160 150 140

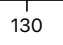
120 110 100 901 f1 (ppm) 80 
(t-BuXPhos)Pd[(4-CF 3$) P h] B r(S 2)$

${ }^{19} \mathrm{~F}$ NMR (471 MHz, $\mathrm{CD}_{2} \mathrm{Cl}_{2}$ )
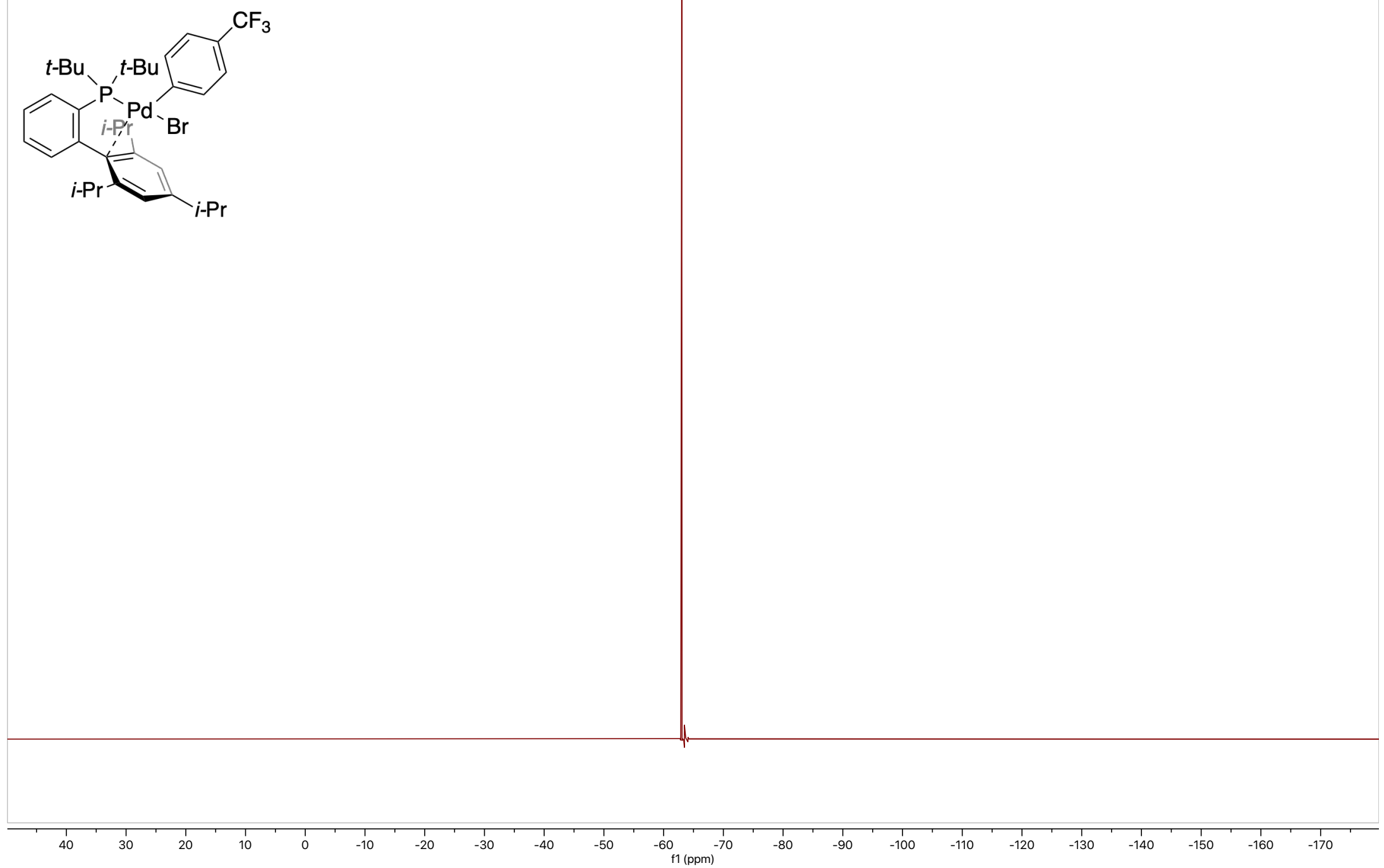
(t-BuXPhos)Pd[(4-CF 3$) \mathrm{Ph}] \mathrm{Br}$ (S2)

${ }^{31} \mathrm{P}$ NMR (203 MHz, $\mathrm{CD}_{2} \mathrm{Cl}_{2}$ )

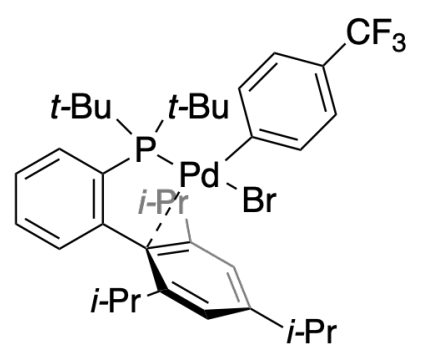

50 140 $130 \quad 120$ 110 100 


\section{( $t$-BuXPhos)Pd[(4-CF 3 )Ph]OTf (S3)}

${ }^{1} \mathrm{H}$ NMR $\left(600 \mathrm{MHz}, \mathrm{CD}_{2} \mathrm{Cl}_{2}\right.$ )

1,111
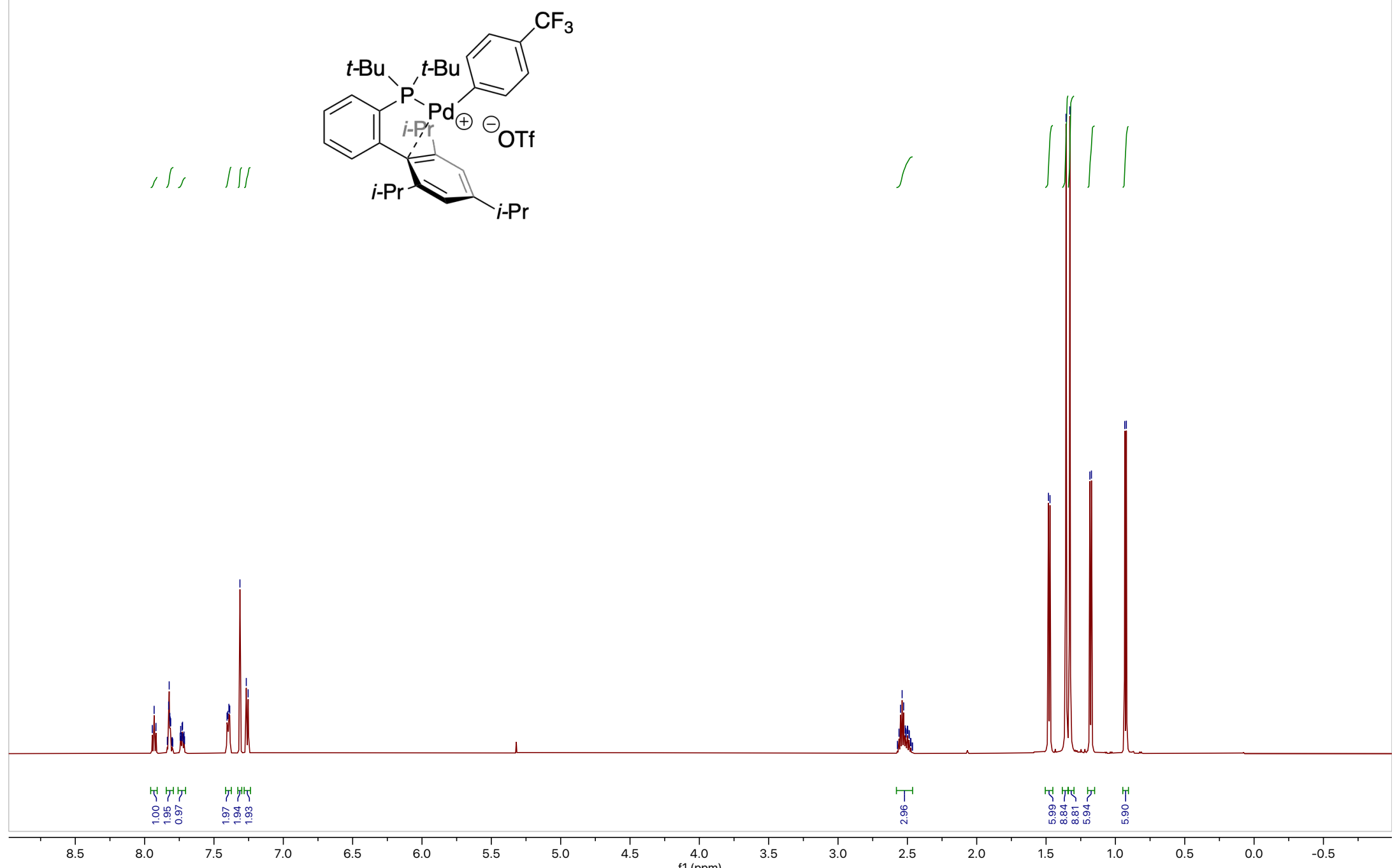


\section{0}

政

(t-BuXPhos)Pd[(4-CF 3$) P h] O T f(S 3)$

${ }^{13} \mathrm{C}$ NMR (151 MHz, $\left.\mathrm{CD}_{2} \mathrm{Cl}_{2}\right)$

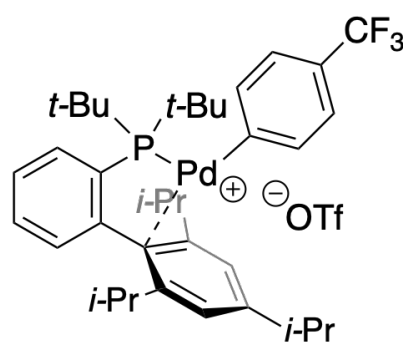

\section{$\mathrm{F}_{3}$}


(t-BuXPhos)Pd[(4-CF 3$) P h] O T f(S 3)$

${ }^{19} \mathrm{~F}$ NMR $\left(565 \mathrm{MHz}, \mathrm{CD}_{2} \mathrm{Cl}_{2}\right)$
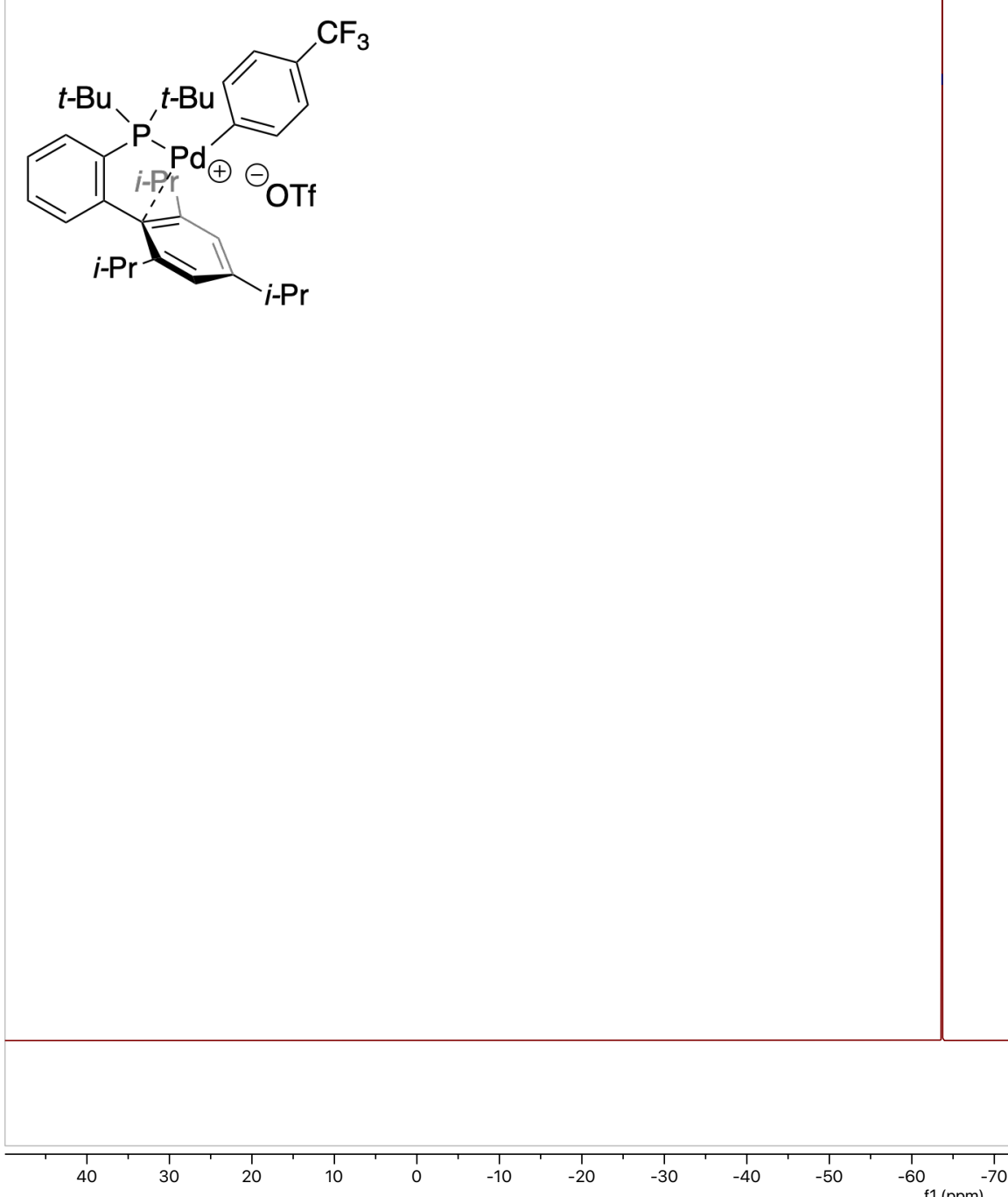

f1 (ppm)

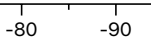

$-100 \quad-110$ 


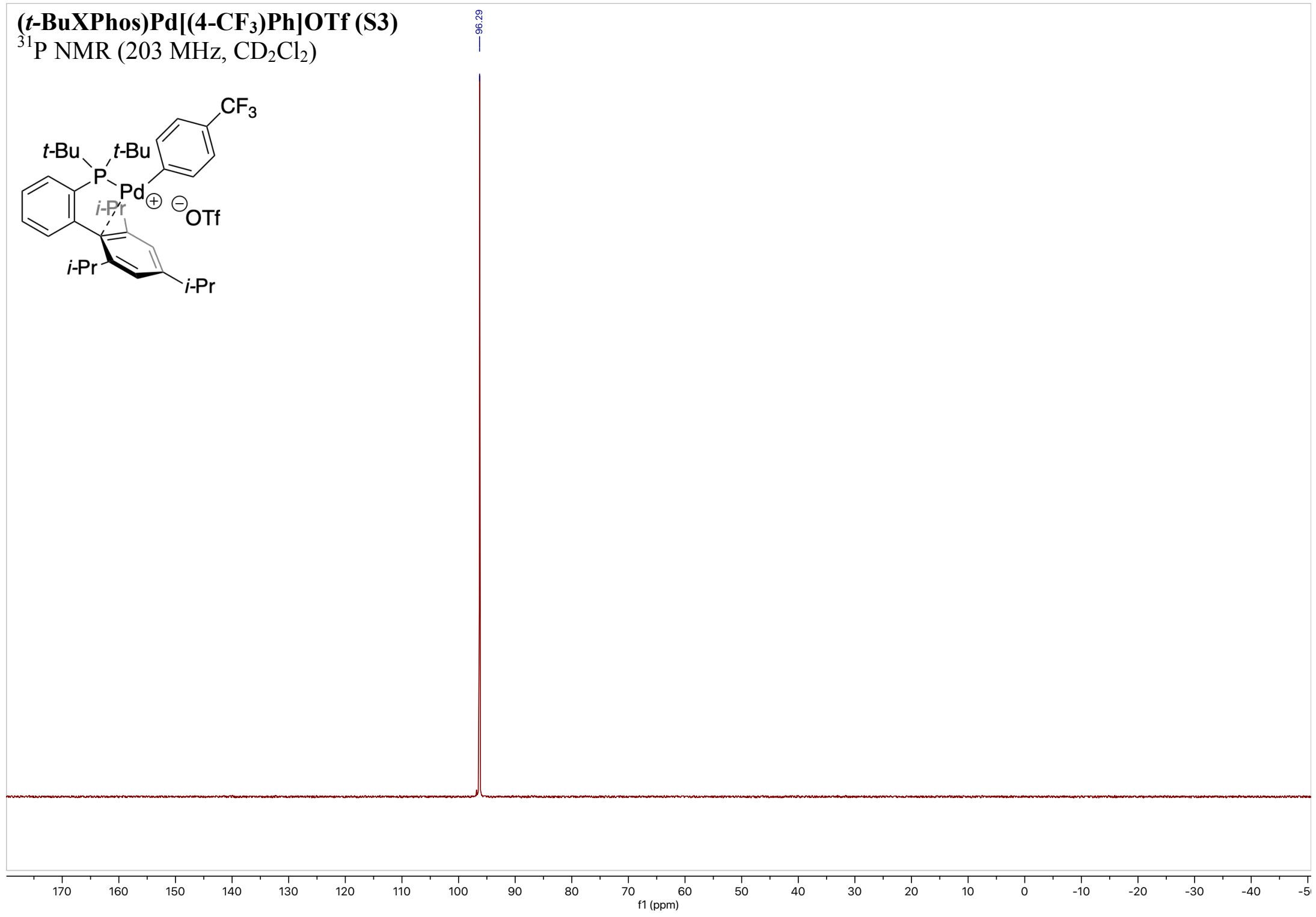




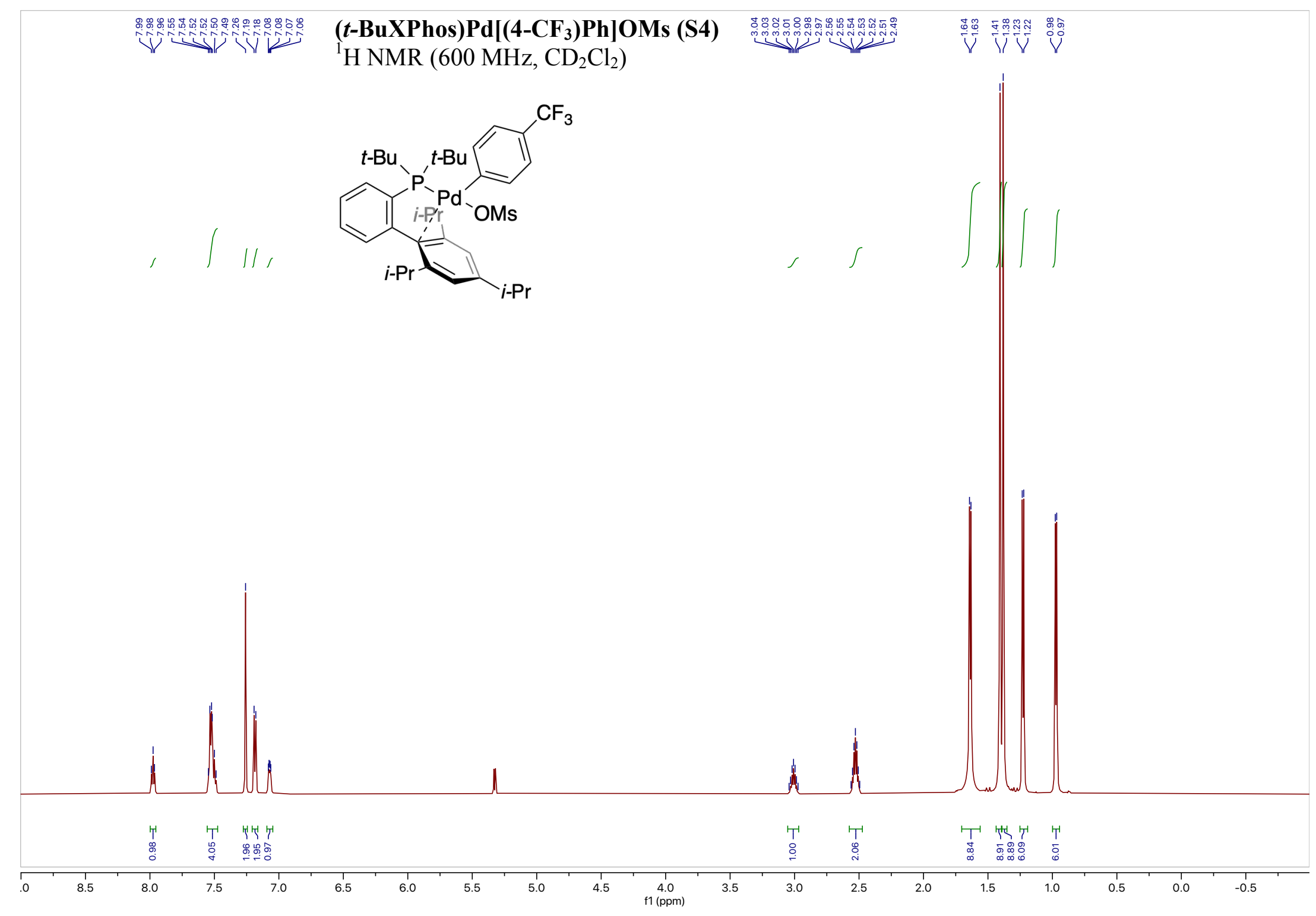




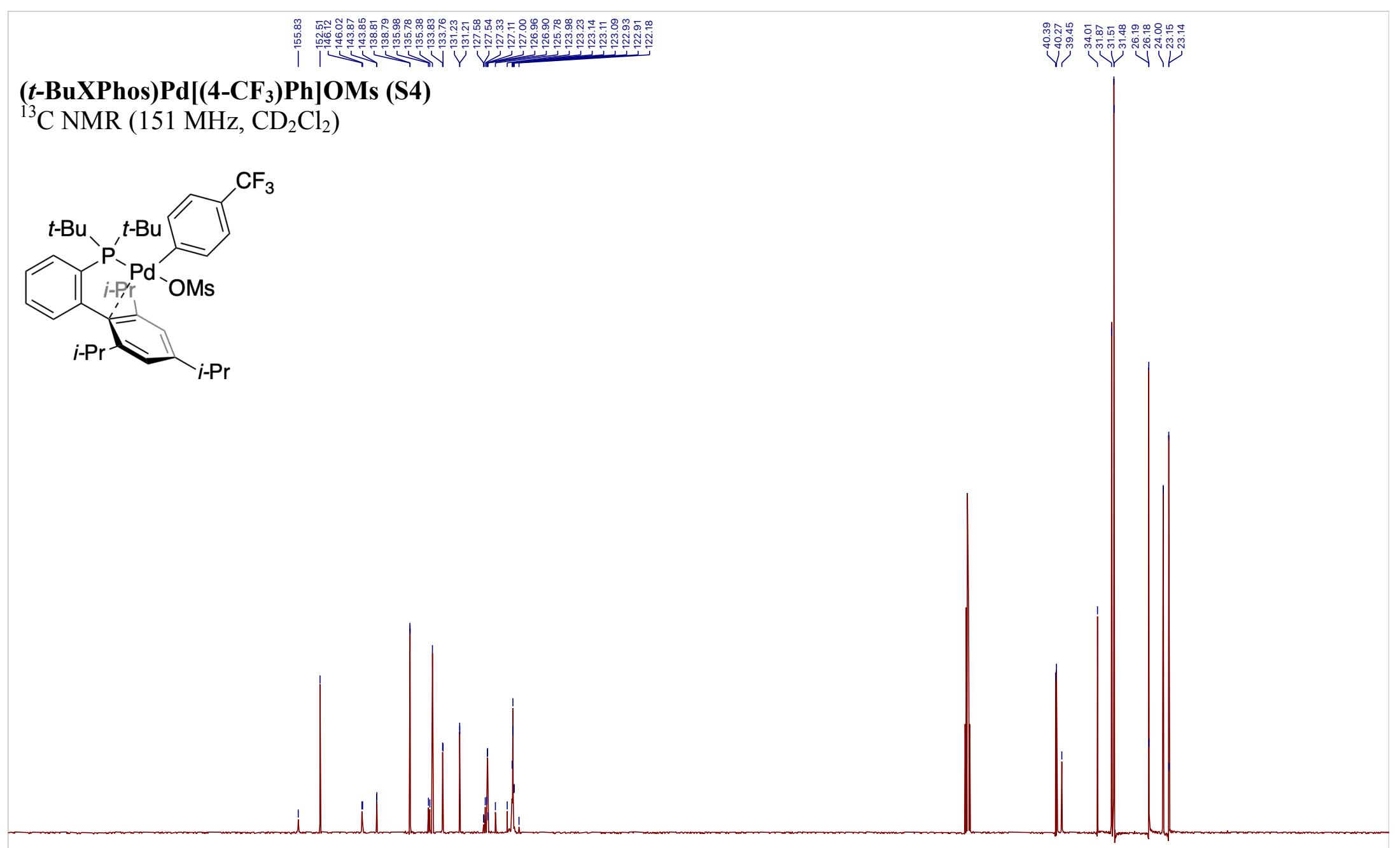


(t-BuXPhos)Pd[(4-CF 3$) P h] O M s ~(S 4)$

${ }^{19} \mathrm{~F}$ NMR $\left(565 \mathrm{MHz}, \mathrm{CD}_{2} \mathrm{Cl}_{2}\right.$ )
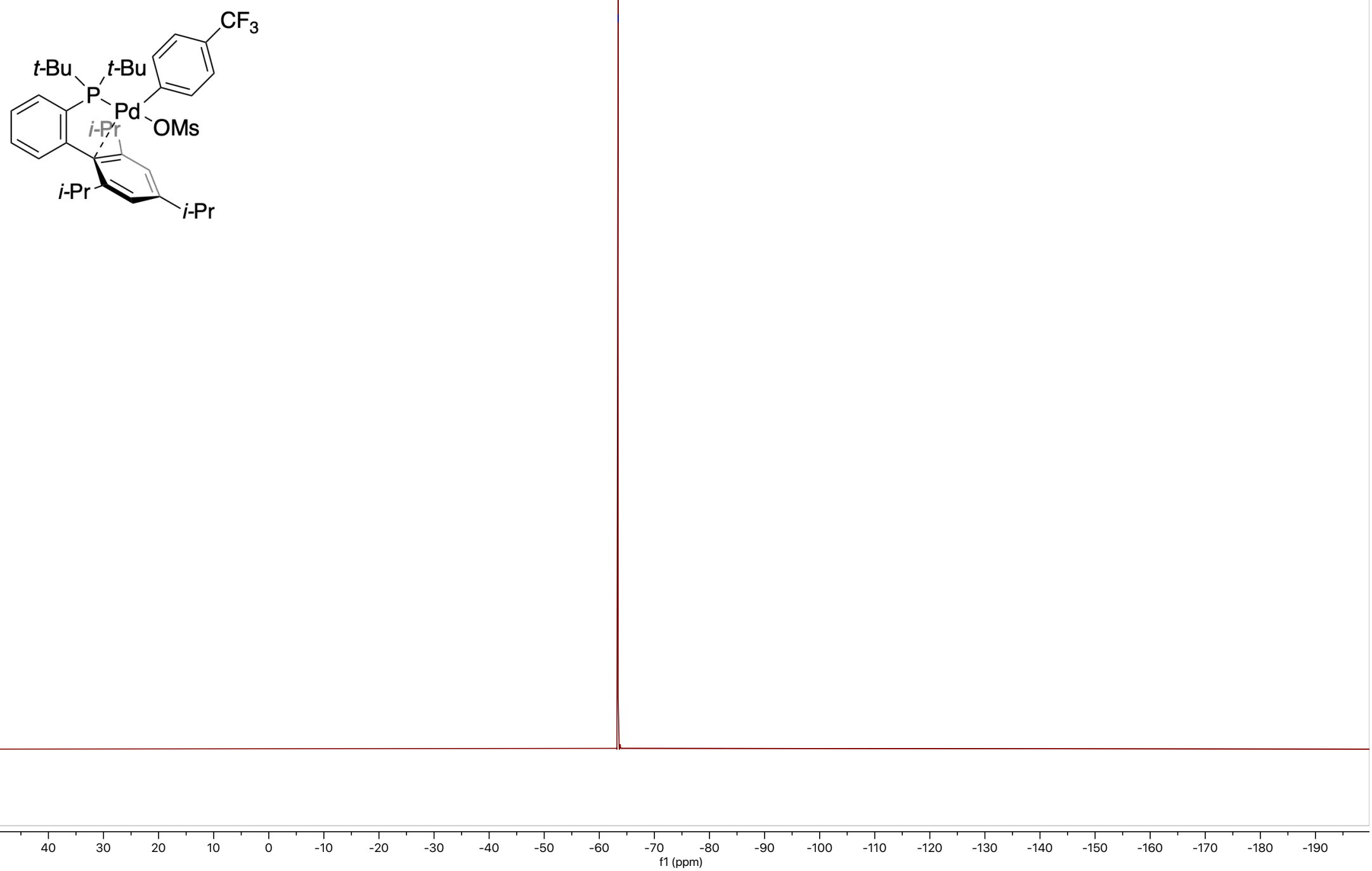
(t-BuXPhos)Pd[(4-CF 3$) P h] O M s ~(S 4)$

${ }^{31} \mathrm{P}$ NMR (202 MHz, $\mathrm{CD}_{2} \mathrm{Cl}_{2}$ )

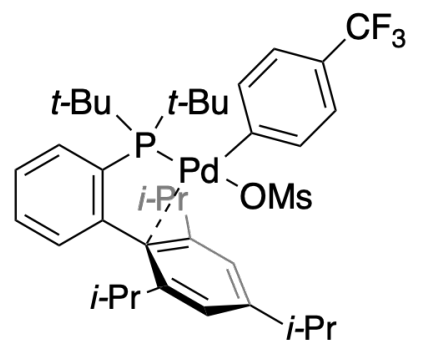

WWy

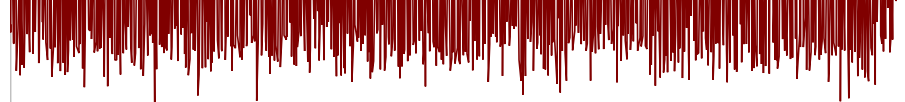

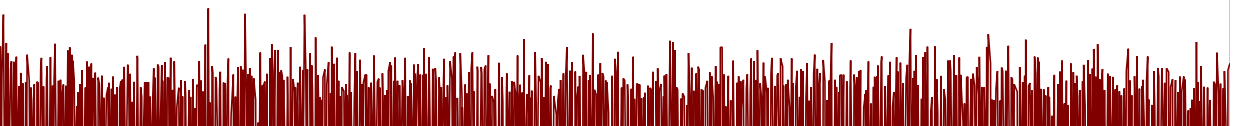

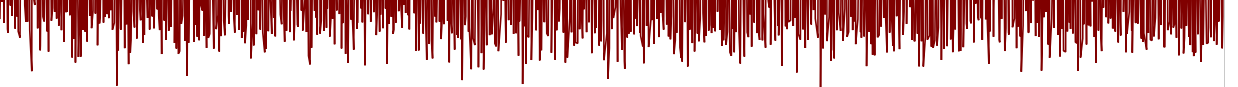

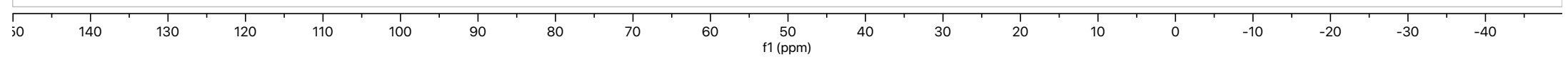




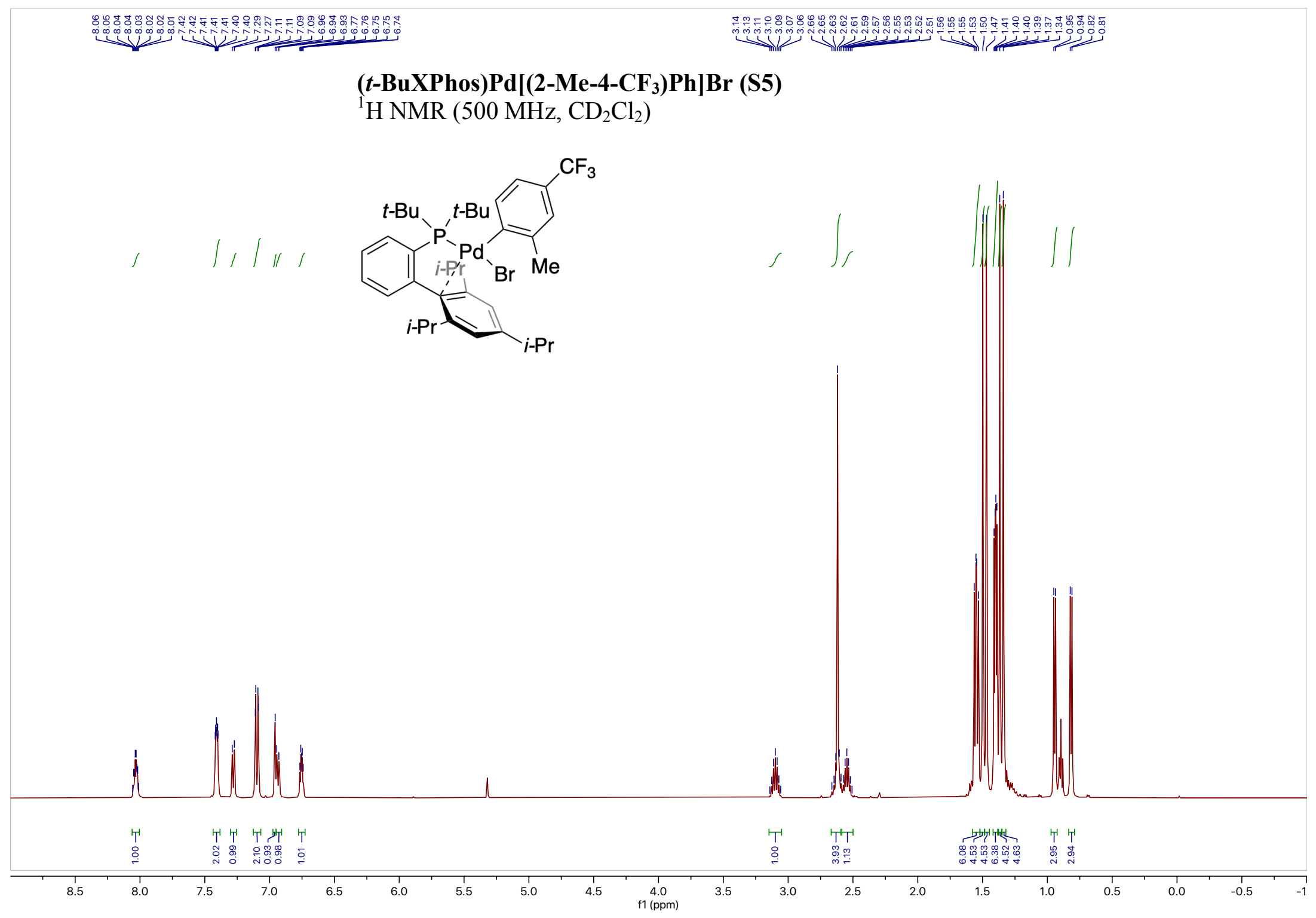




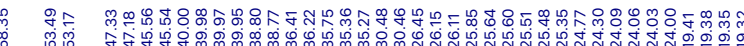

$\sqrt{1}$

(t-BuXPhos)Pd[(2-Me-4-CF 3 )Ph]Br (S5)

${ }^{13} \mathrm{C}$ NMR $\left(126 \mathrm{MHz}, \mathrm{CD}_{2} \mathrm{Cl}_{2}\right)$

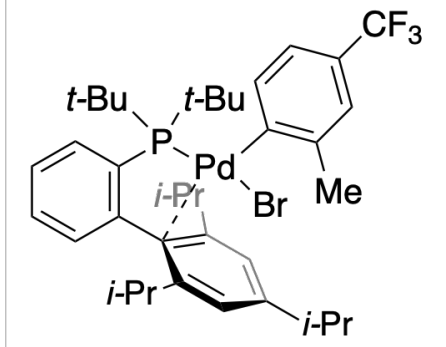

30

170 160

150

140

130

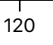

110

100 90 f1 (ppm) 80 70 60 50 40 30 20 10 
( $t$-BuXPhos)Pd[(2-Me-4-CF 3 )Ph]Br (S5)

${ }^{19} \mathrm{~F}$ NMR (471 MHz, $\mathrm{CD}_{2} \mathrm{Cl}_{2}$ )
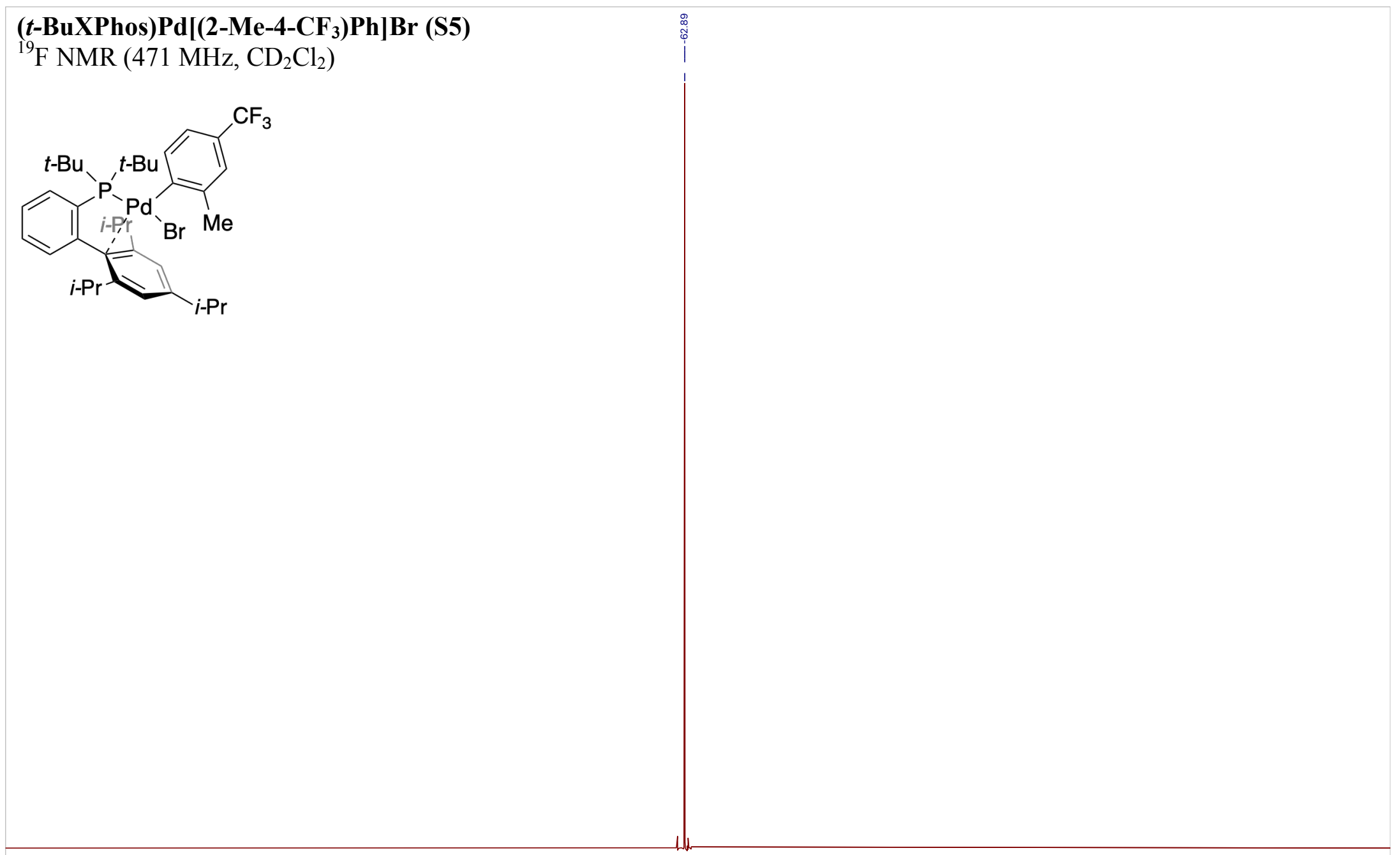

0
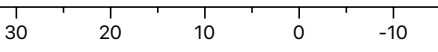

1
-20

$\begin{array}{ll}-40 & -50\end{array}$

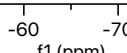


(t-BuXPhos)Pd[(2-Me-4-CF 3$) P h] B r(S 5)$

${ }^{31} \mathrm{P}$ NMR (203 MHz, $\mathrm{CD}_{2} \mathrm{Cl}_{2}$ )

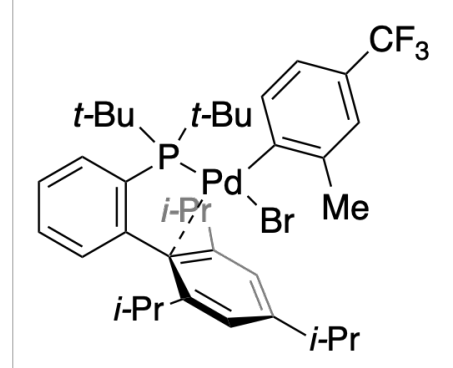

50

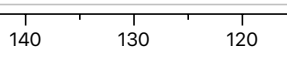
110 100 90 80 $70 \quad 60$ 50
$\mathrm{f} 1(\mathrm{ppm})$ 


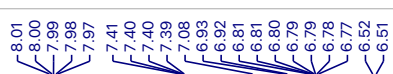

(t-BuXPhos)Pd[(4-OMe)Ph]Cl (S6)

${ }^{1} \mathrm{H}$ NMR $\left(500 \mathrm{MHz}, \mathrm{CD}_{2} \mathrm{Cl}_{2}\right.$ )
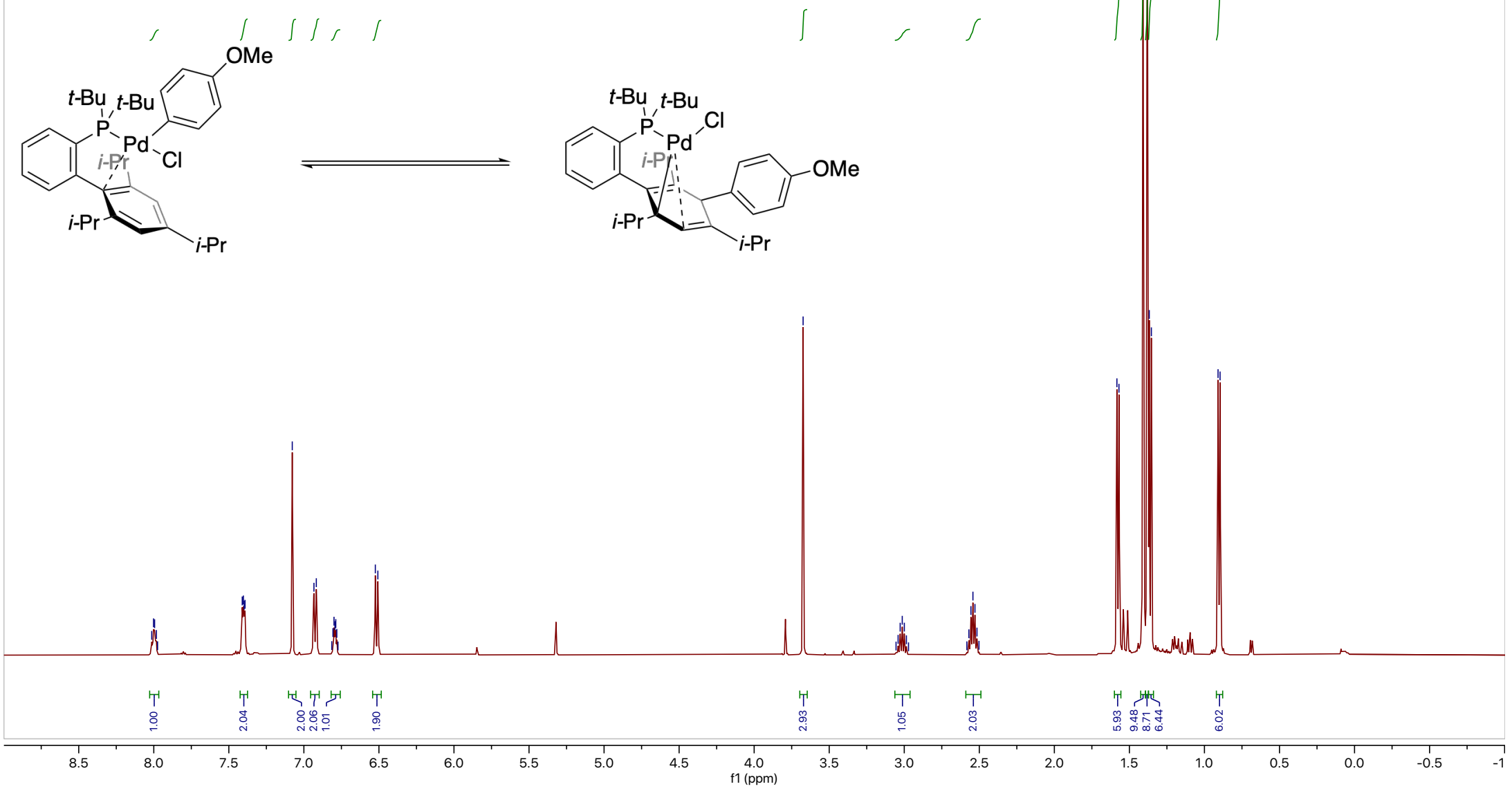


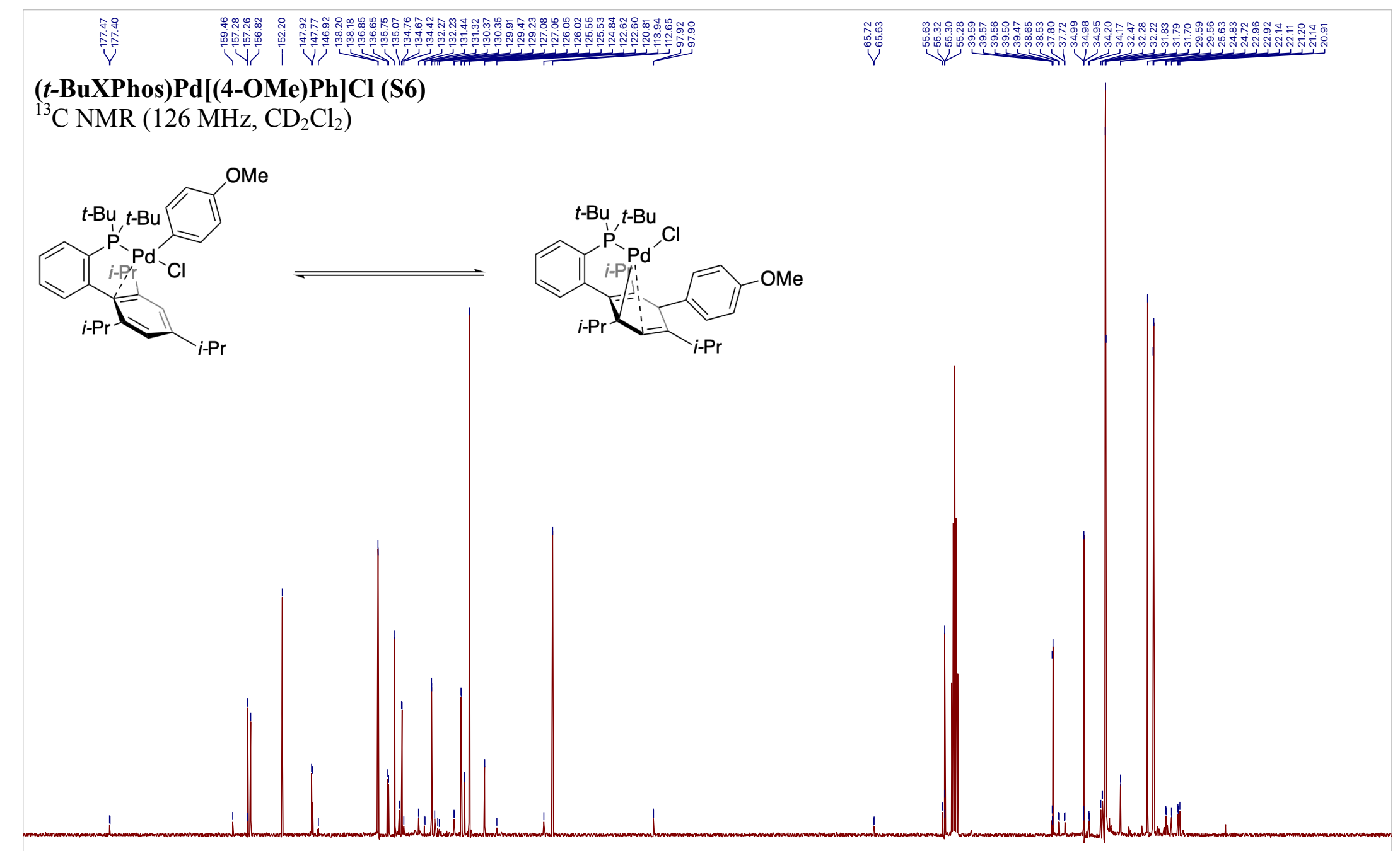


(t-BuXPhos)Pd[(4-OMe)Ph]Cl (S6)

${ }^{31} \mathrm{P}$ NMR (203 MHz, $\left.\mathrm{CD}_{2} \mathrm{Cl}_{2}\right)$
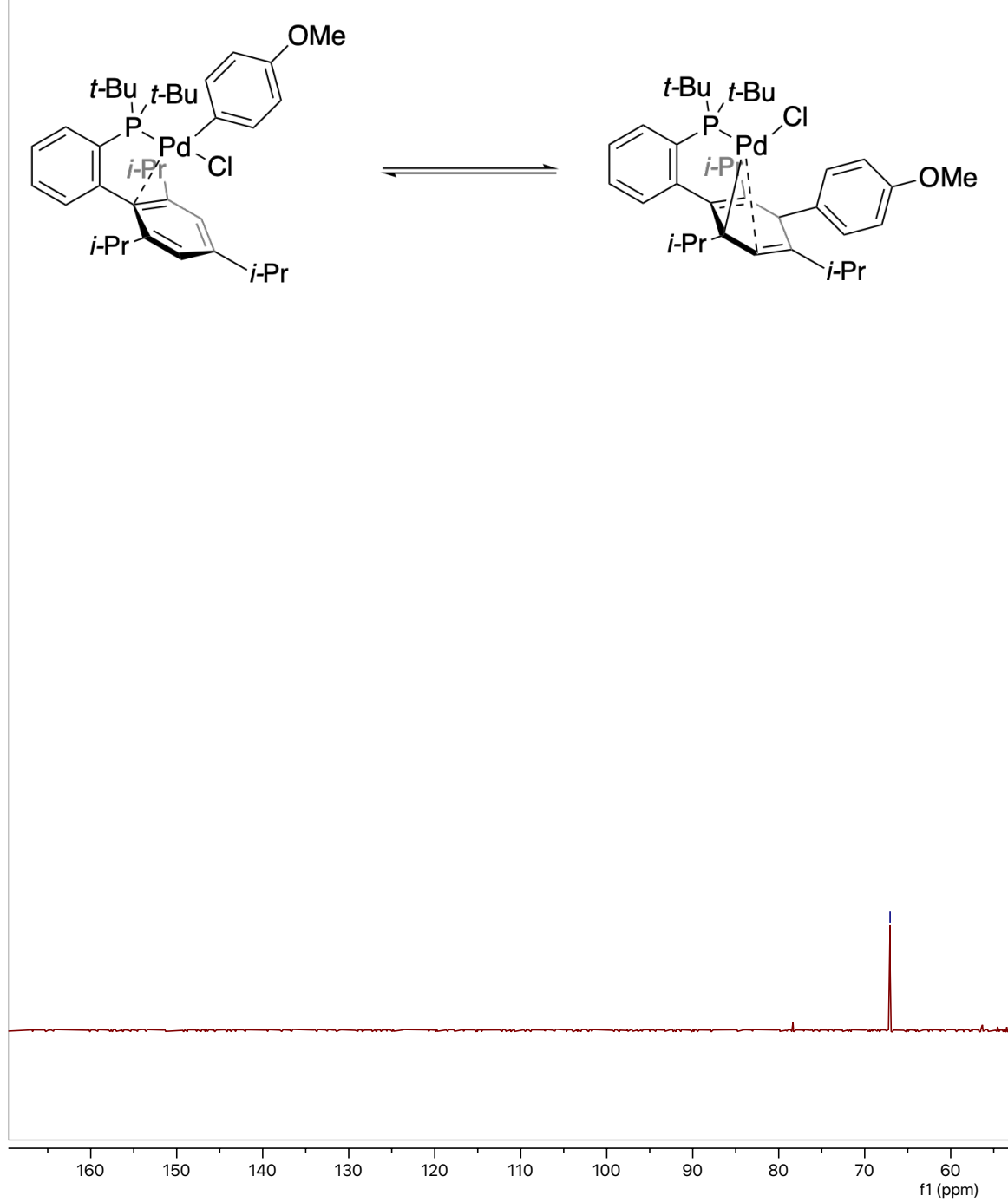

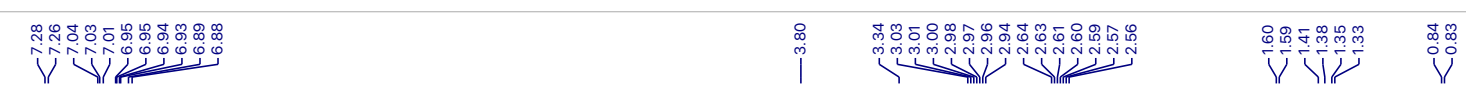

(t-BuBrettPhos)Pd[(4-CF 3$) \mathbf{P h}] \mathrm{Cl}$ (S7)

${ }^{1} \mathrm{H}$ NMR (500 MHz, $\mathrm{CD}_{2} \mathrm{Cl}_{2}$ )
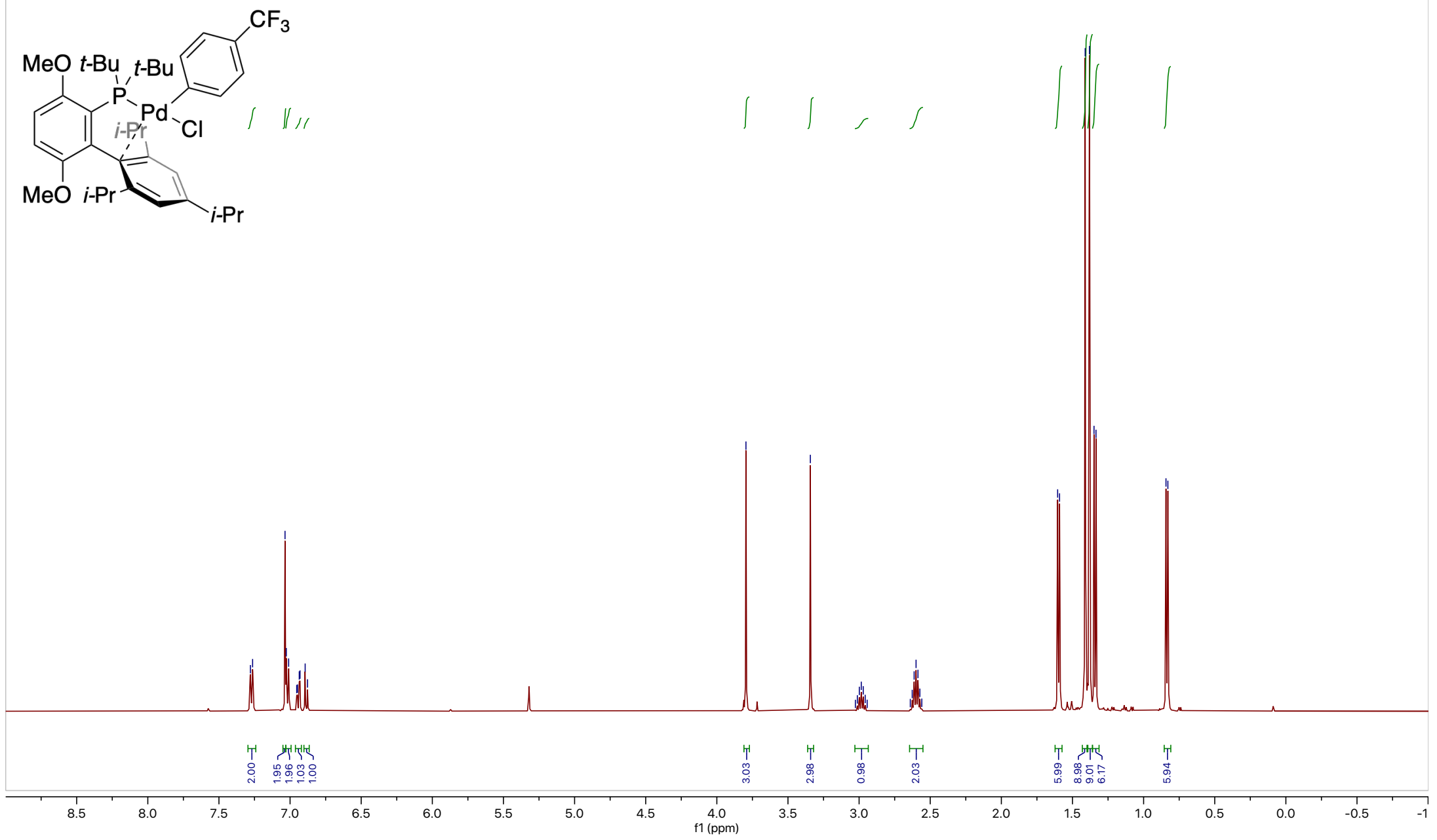


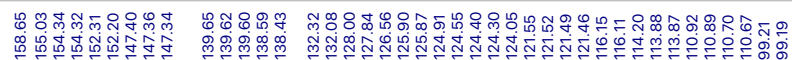

UV

(t-BuBrettPhos)Pd[(4-CF $) \mathbf{P h}] \mathrm{Cl}(\mathrm{S} 7)$

${ }^{13} \mathrm{C}$ NMR (126 MHz, $\mathrm{CD}_{2} \mathrm{Cl}_{2}$ )
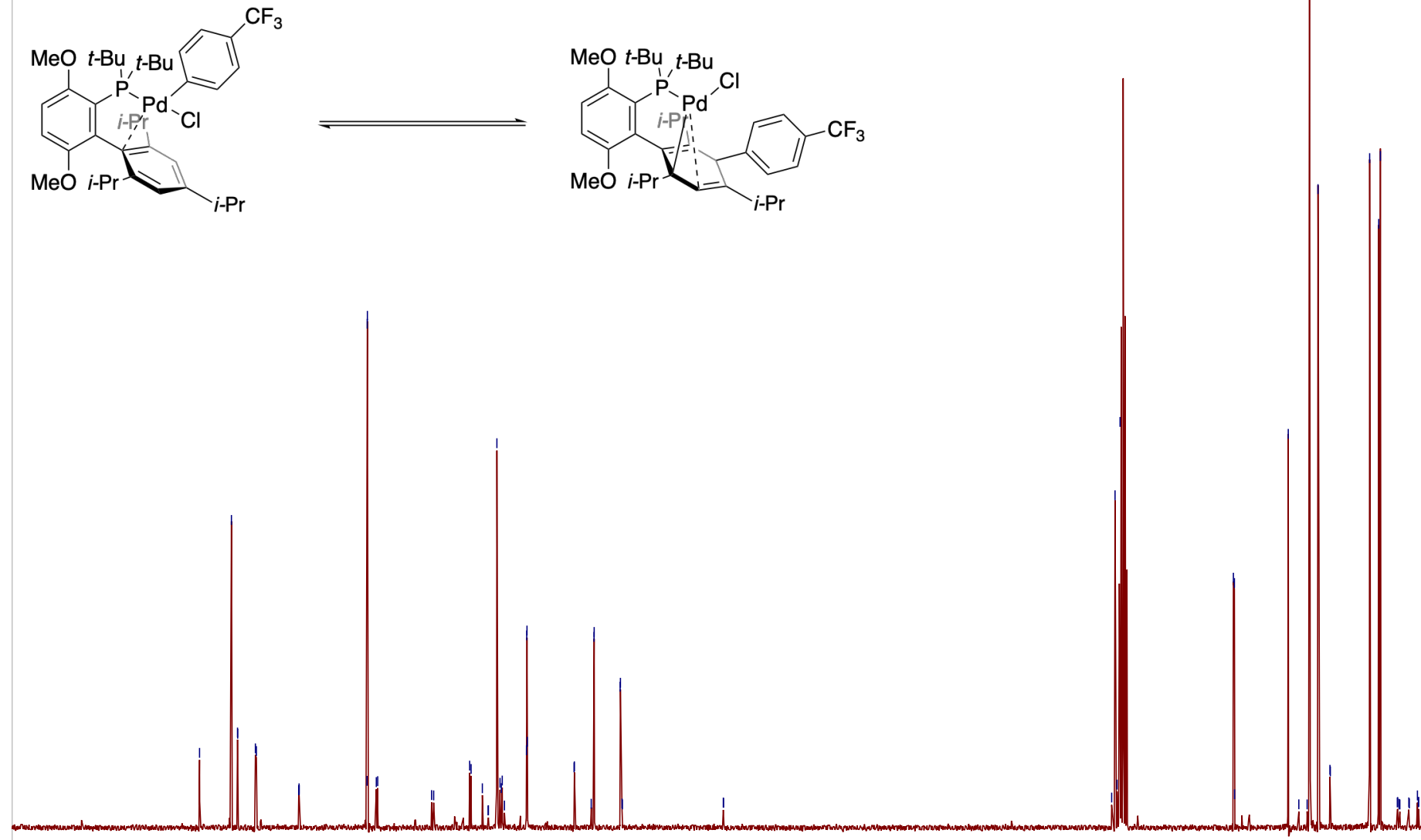
(t-BuBrettPhos)Pd[(4-CF 3$) \mathrm{Ph}] \mathrm{Cl}$ (S7)

${ }^{19} \mathrm{~F}$ NMR (471 MHz, $\mathrm{CD}_{2} \mathrm{Cl}_{2}$ )
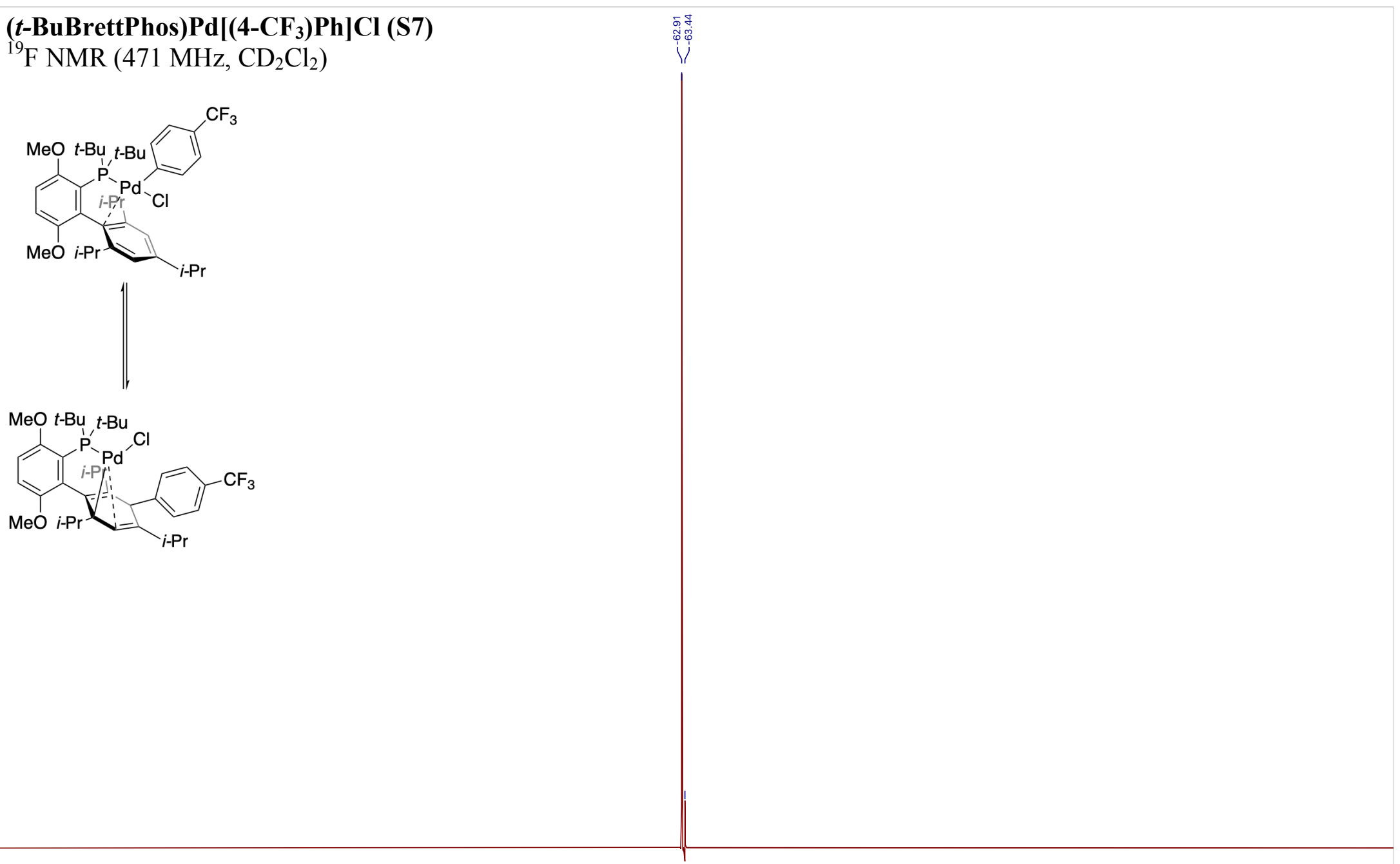
(t-BuBrettPhos)Pd[(4-CF 3$) \mathrm{Ph}] \mathrm{Cl}$ (S7)

${ }^{31} \mathrm{P}$ NMR (203 MHz, $\mathrm{CD}_{2} \mathrm{Cl}_{2}$ )
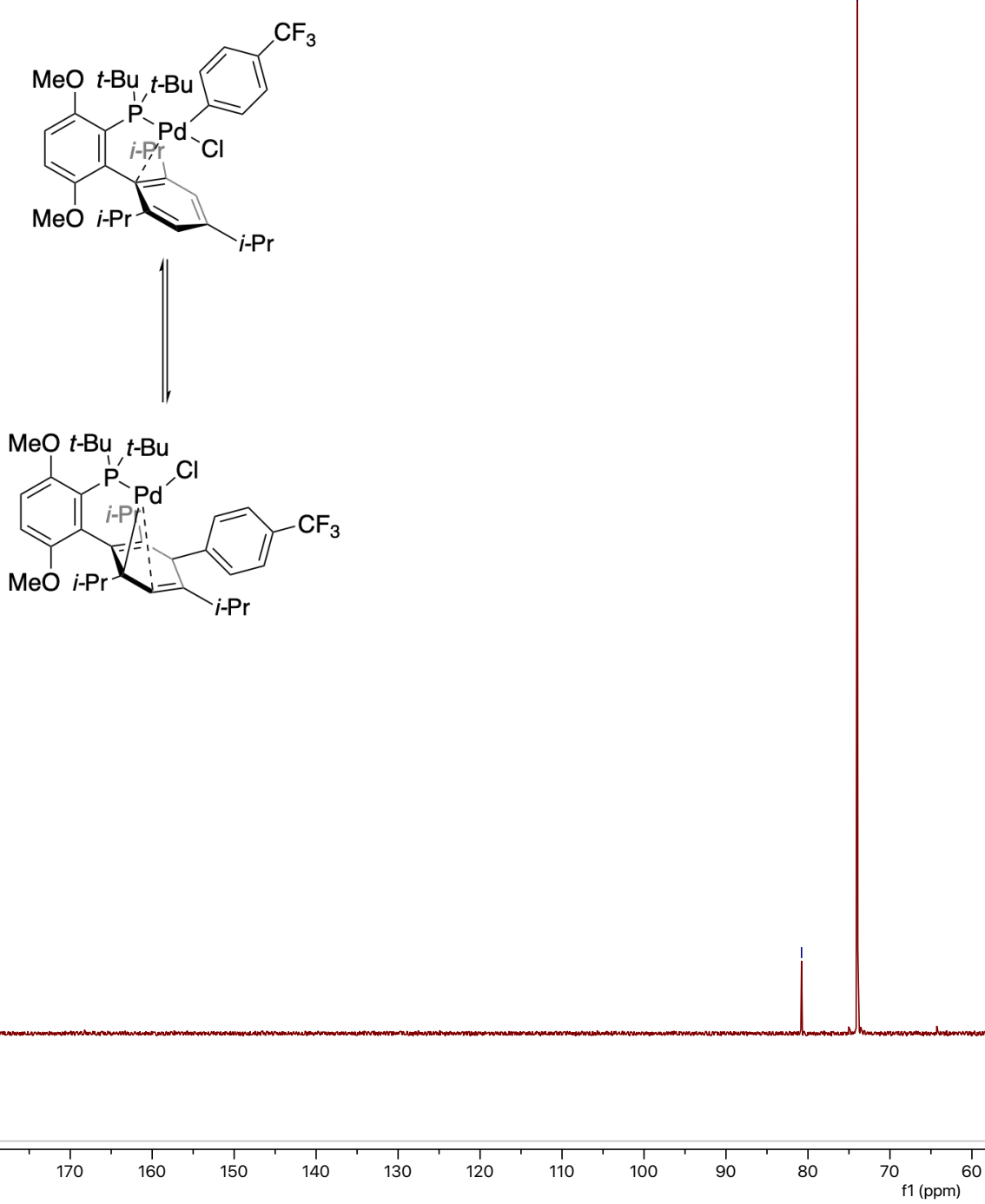


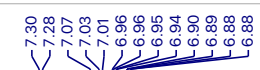

(t-BuBrettPhos)Pd[(4-CF 3$) \mathrm{Ph}] \mathrm{Br}$ (S8)

${ }^{1} \mathrm{H} \mathrm{NMR}\left(500 \mathrm{MHz}, \mathrm{CD}_{2} \mathrm{Cl}_{2}\right.$ )
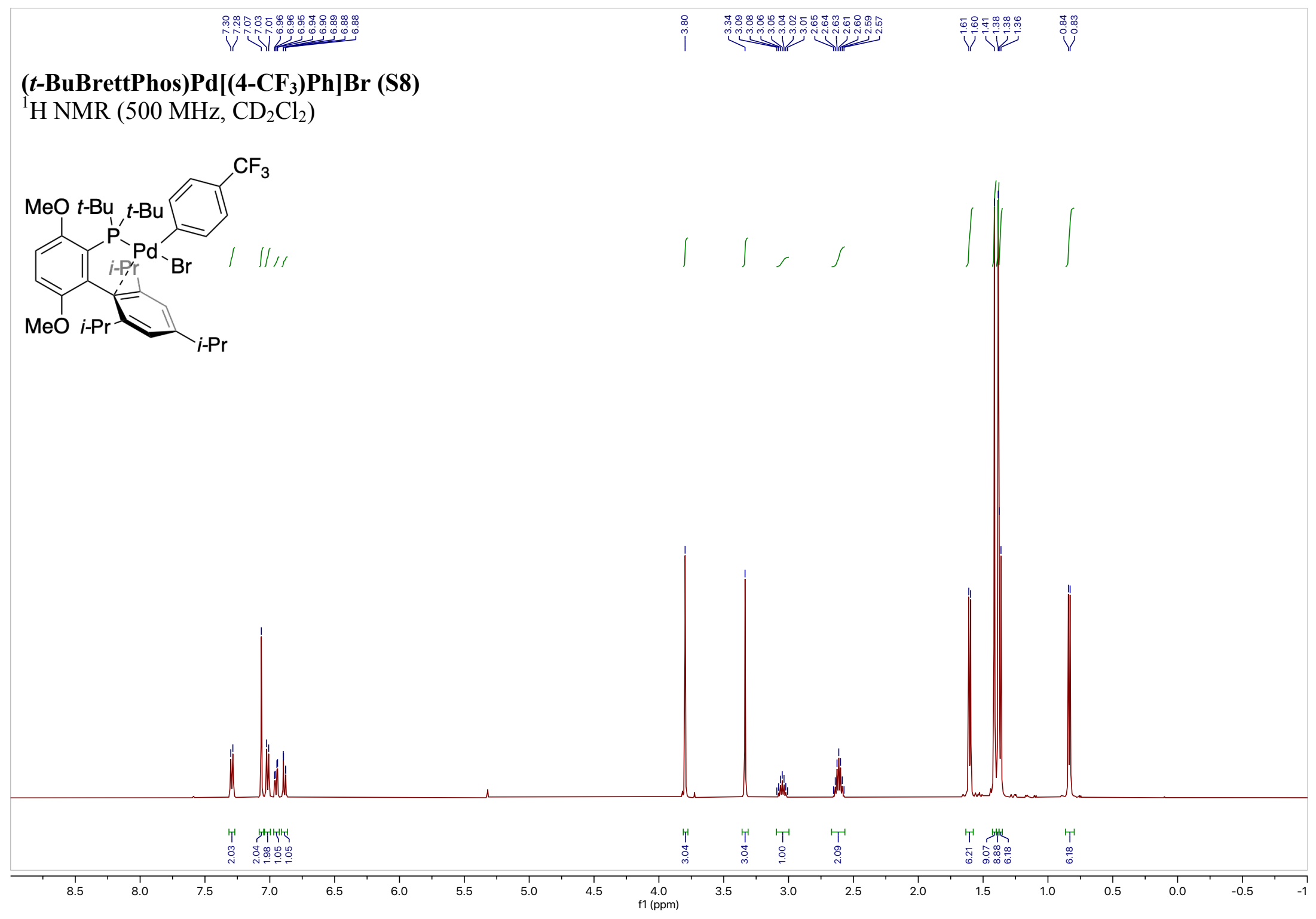

(t-BuBrettPhos)Pd[(4-CF 3$) \mathbf{P h}] \mathrm{Br}$ (S8)

${ }^{13} \mathrm{C}$ NMR $\left(126 \mathrm{MHz}, \mathrm{CD}_{2} \mathrm{Cl}_{2}\right)$
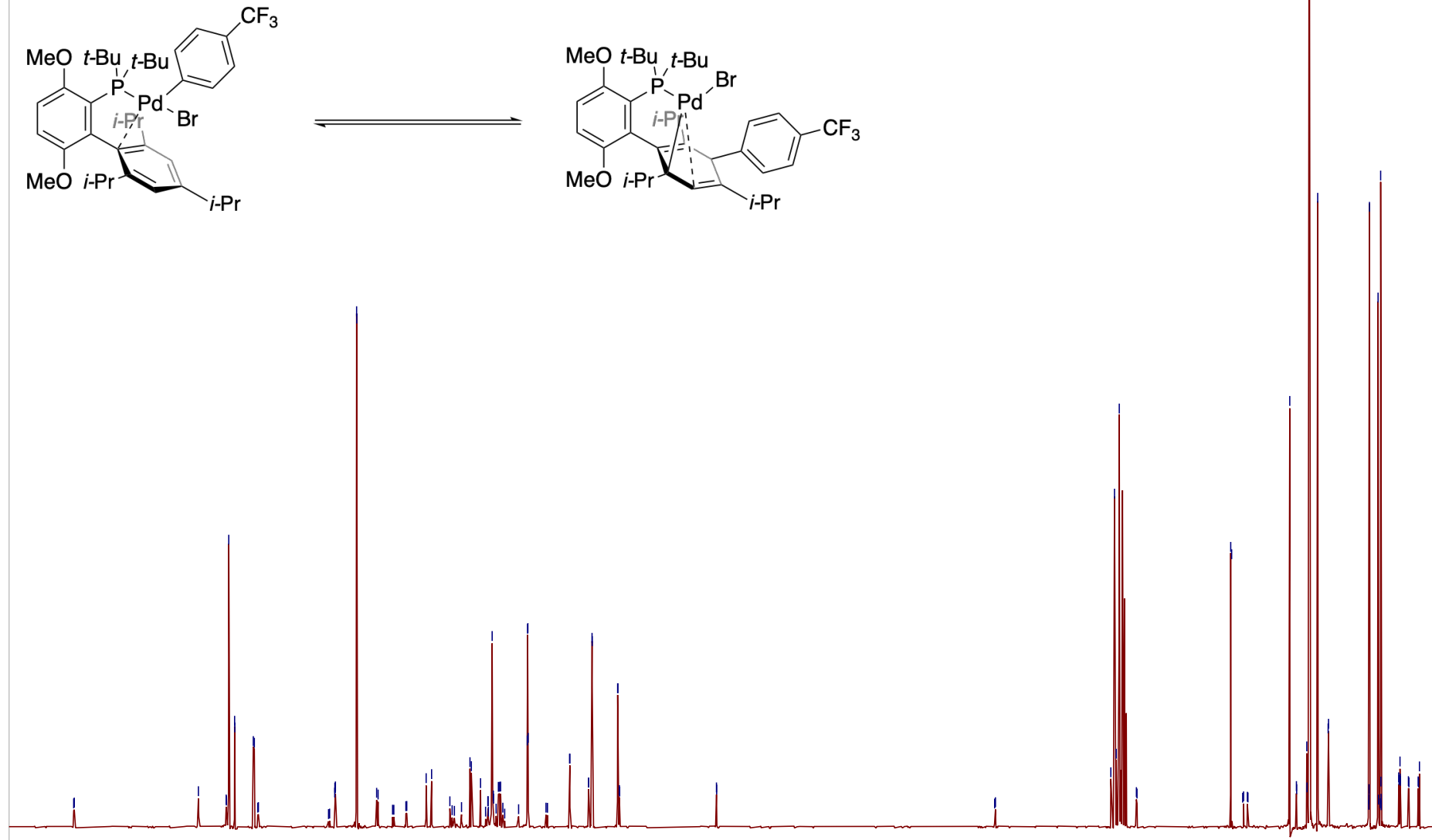
(t-BuBrettPhos)Pd[(4-CF 3$) \mathbf{P h}] \mathrm{Br}$ (S8)

${ }^{19} \mathrm{~F}$ NMR (471 MHz, $\mathrm{CD}_{2} \mathrm{Cl}_{2}$ )
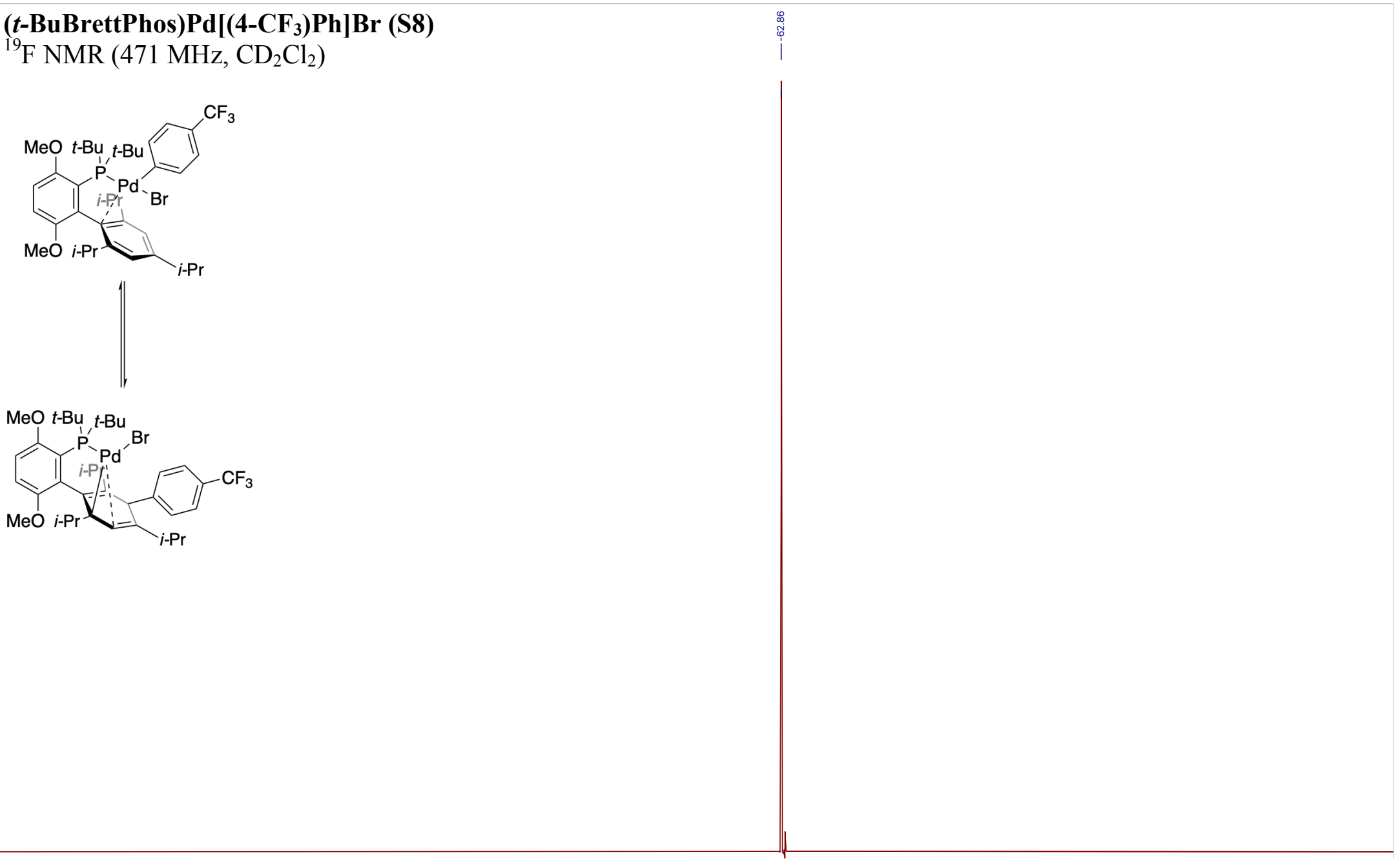
(t-BuBrettPhos)Pd[(4-CF 3 ) Ph]Br (S8)

${ }^{31} \mathrm{P}$ NMR (203 MHz, $\mathrm{CD}_{2} \mathrm{Cl}_{2}$ )
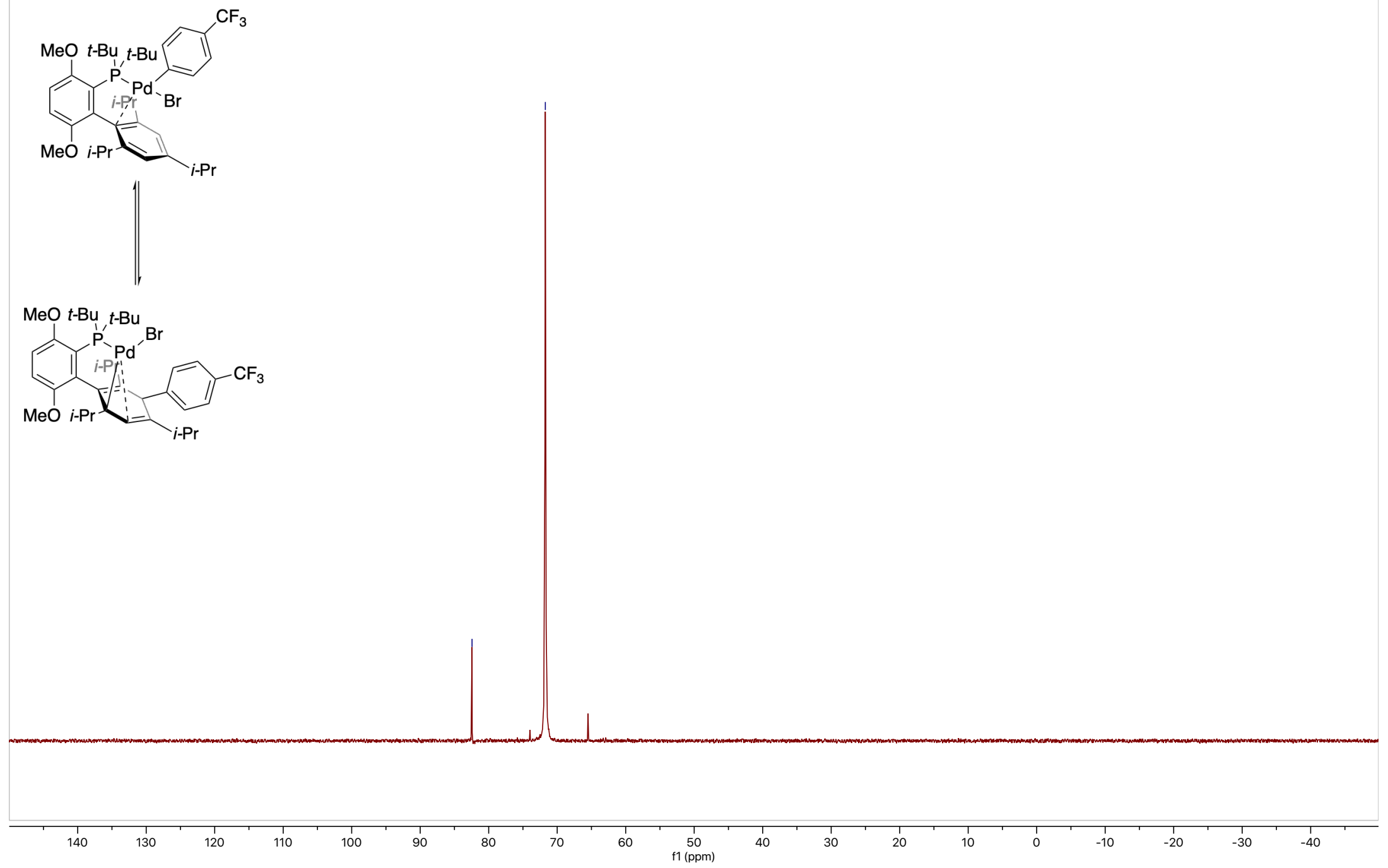

S73 


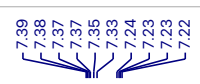

(t-BuBrettPhos)Pd[(4-CF 3$)$ Ph]OTf (S9)

${ }^{1} \mathrm{H}$ NMR $\left(600 \mathrm{MHz}, \mathrm{CD}_{2} \mathrm{Cl}_{2}\right.$ )

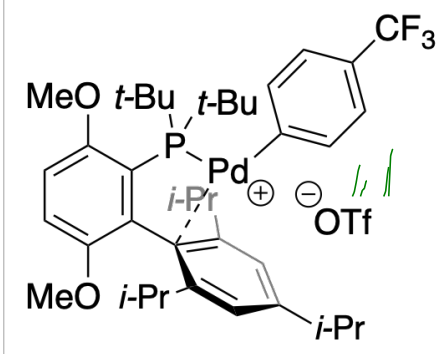

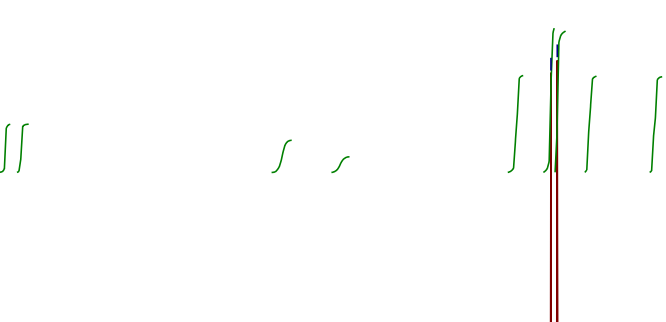

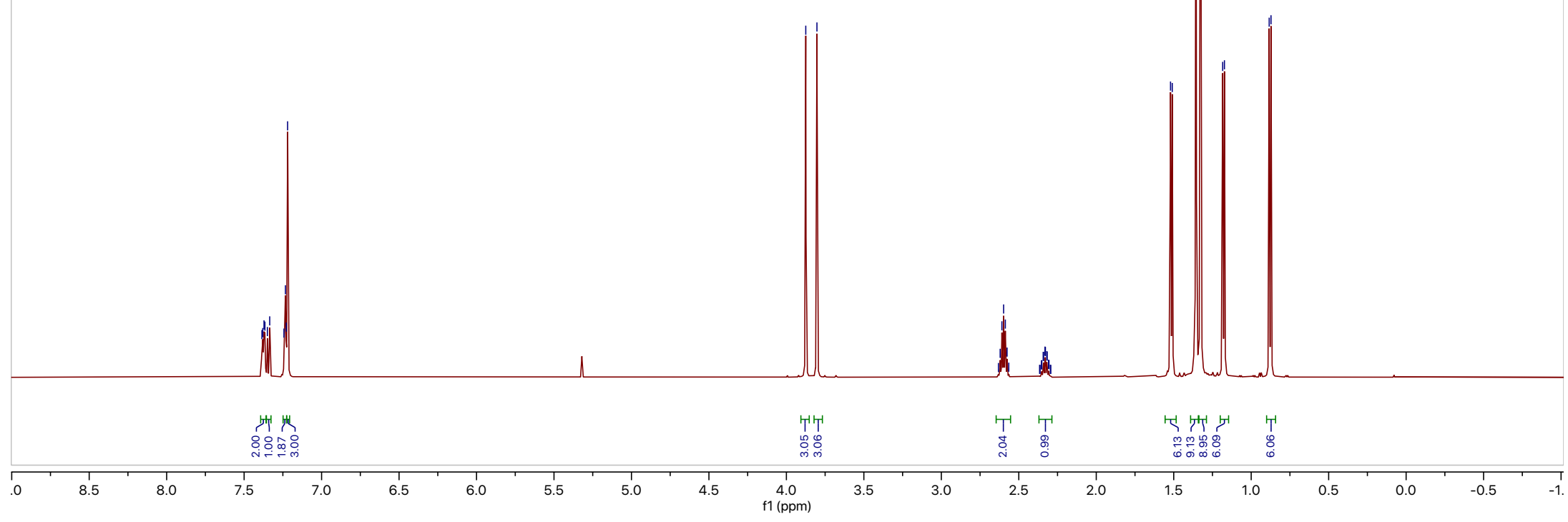




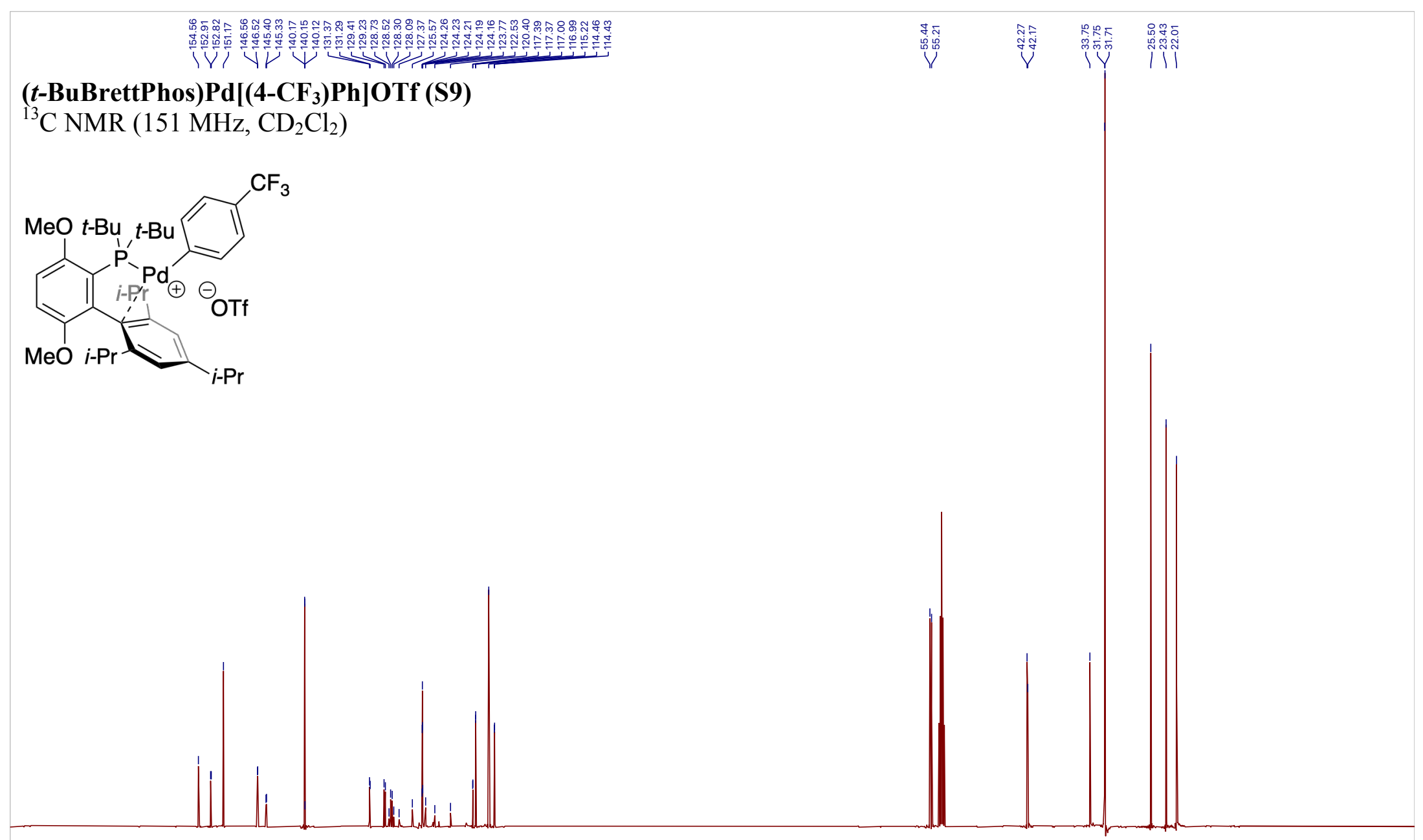


(t-BuBrettPhos)Pd[(4-CF 3 )Ph]OTf (S9)

${ }^{19} \mathrm{~F}$ NMR (565 MHz, $\mathrm{CD}_{2} \mathrm{Cl}_{2}$ )
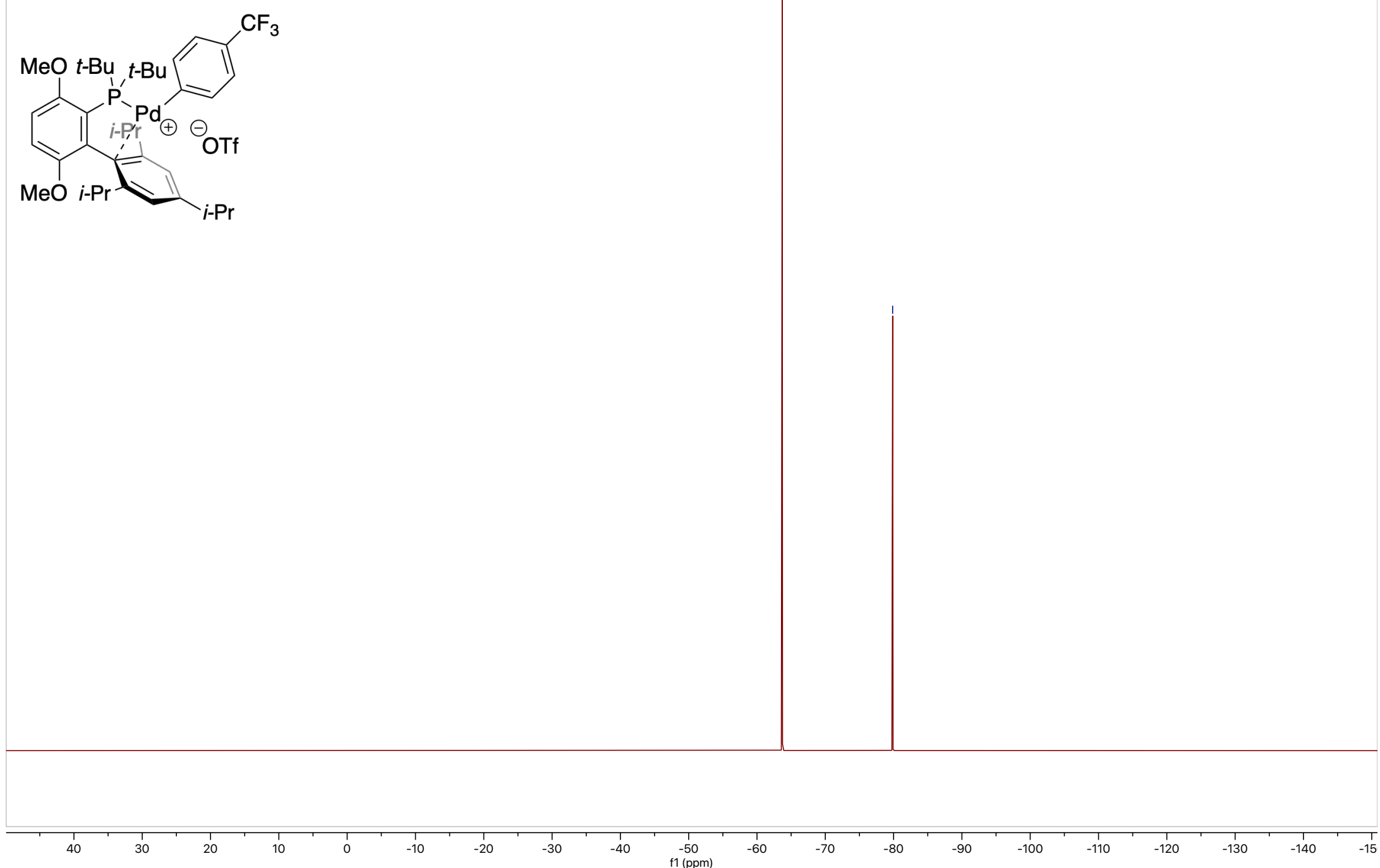


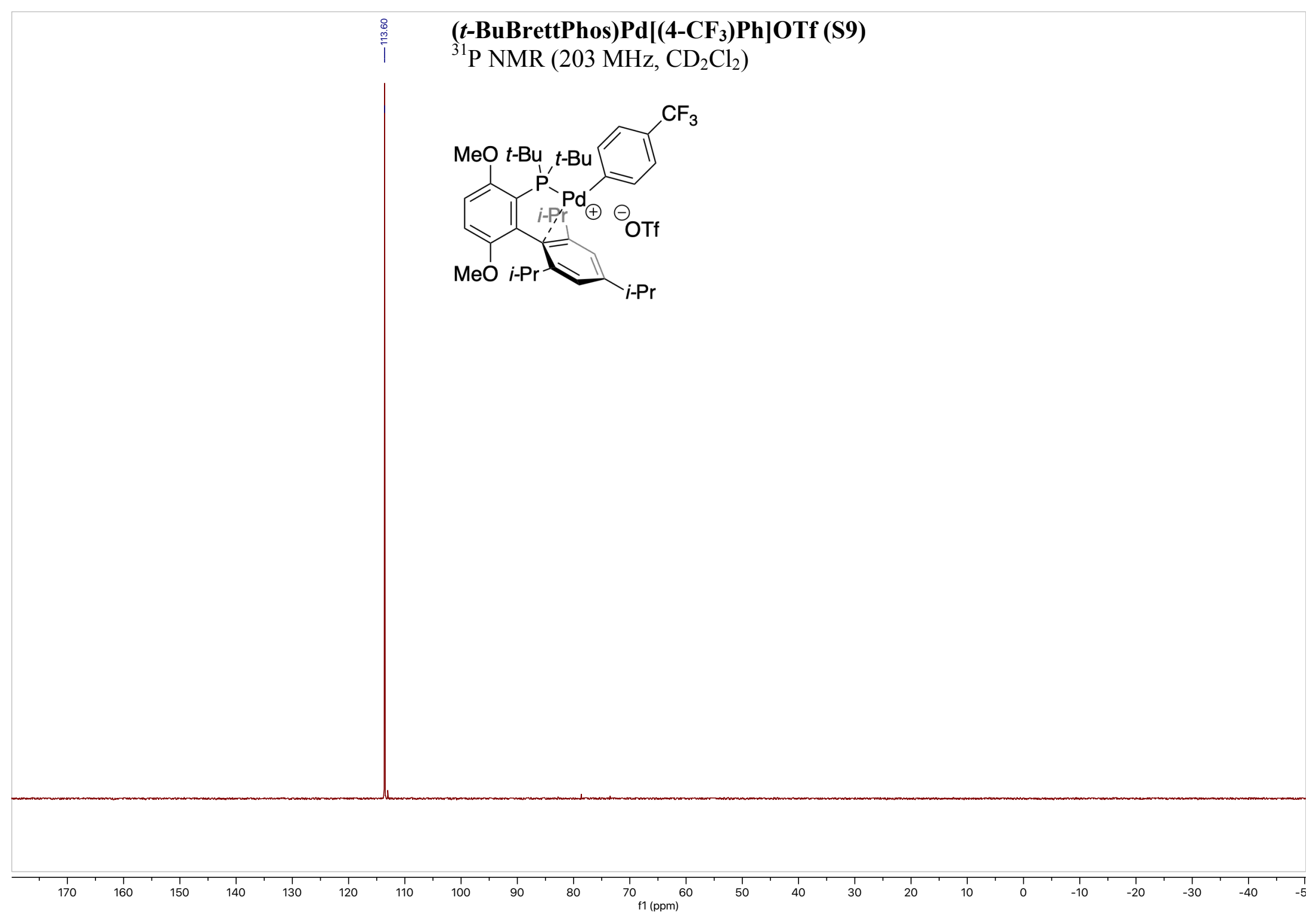




\section{بـ}

(t-BuXPhos)Pd(X14)I (S10)

${ }^{1} \mathrm{H}$ NMR $\left(600 \mathrm{MHz}, \mathrm{CD}_{2} \mathrm{Cl}_{2}\right.$ )

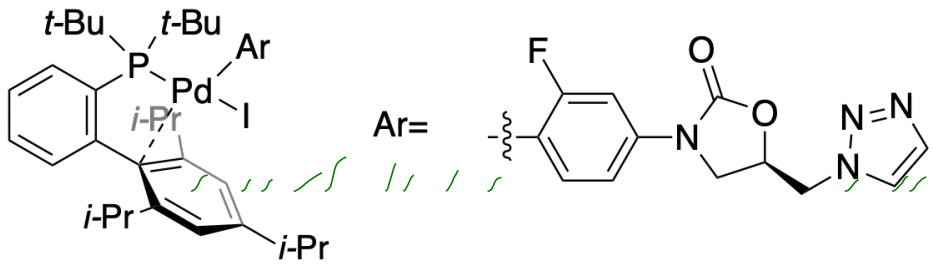

$\underbrace{ن}$

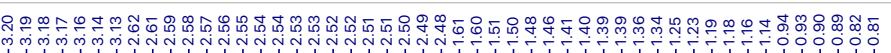
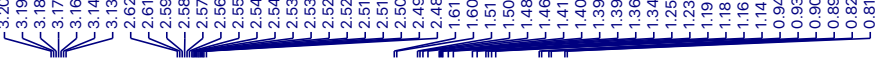
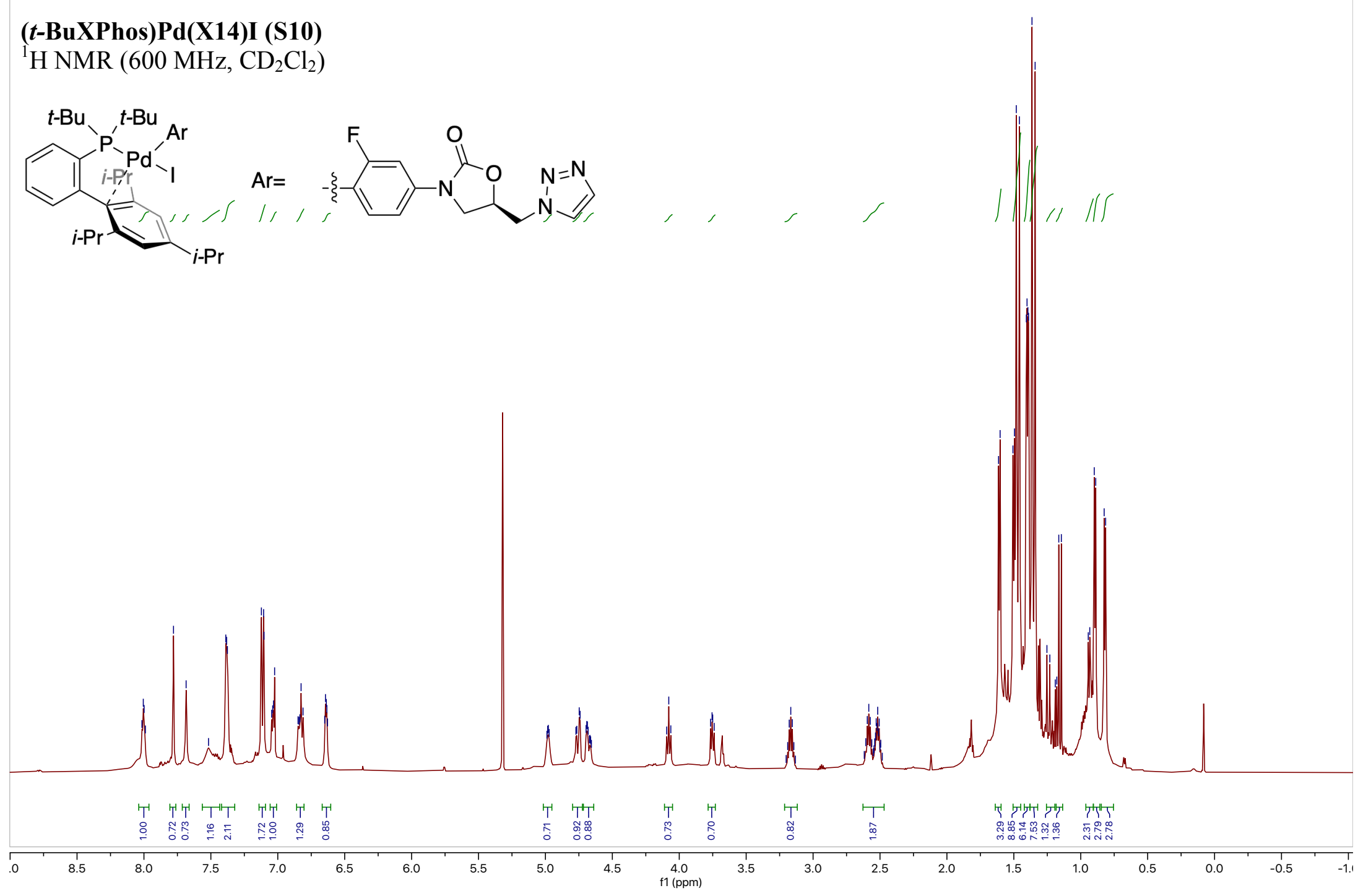


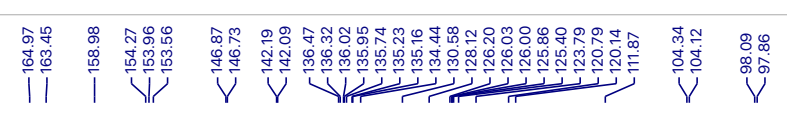

(t-BuXPhos)Pd(X14)I (S10)

${ }^{13} \mathrm{C}$ NMR (151 MHz, $\mathrm{CD}_{2} \mathrm{Cl}_{2}$ )
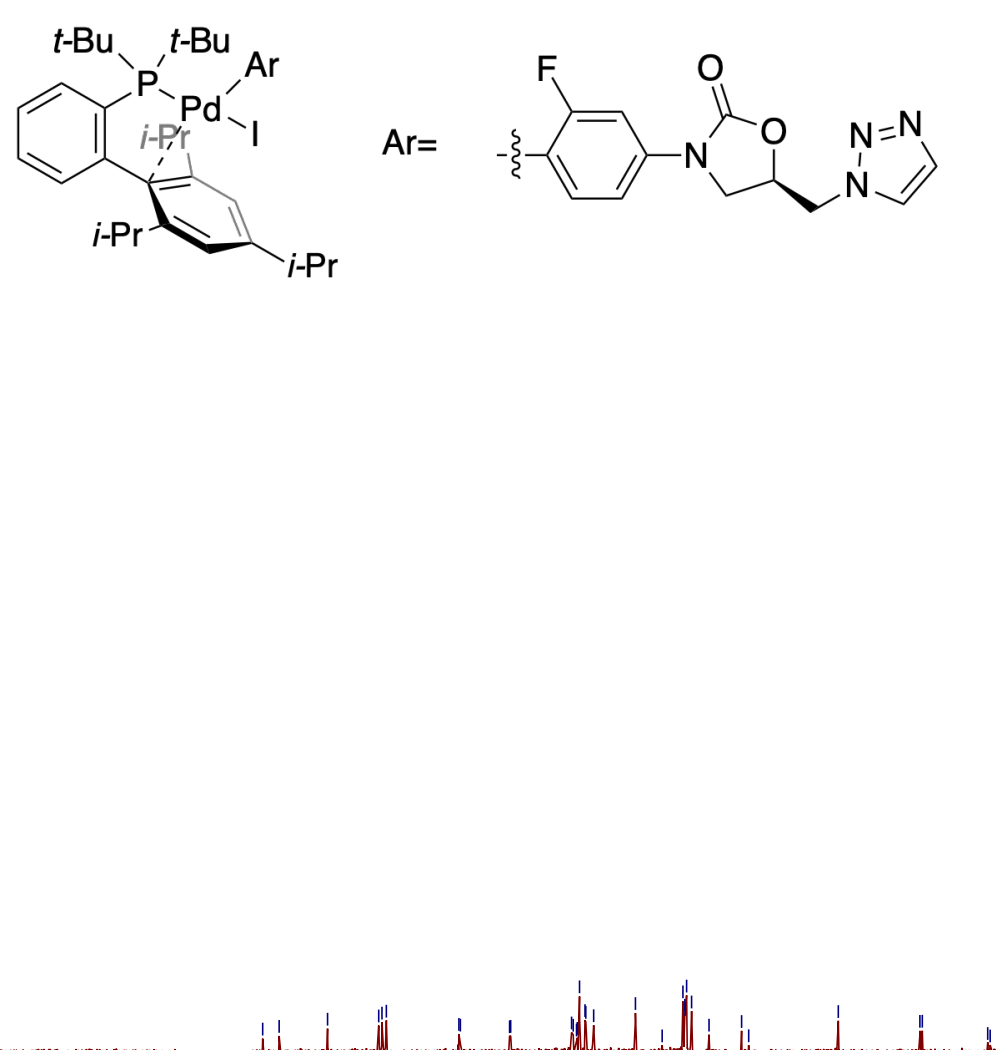
(t-BuXPhos)Pd(X14)I (S10)

${ }^{19} \mathrm{~F}$ NMR (565 MHz, $\mathrm{CD}_{2} \mathrm{Cl}_{2}$ )

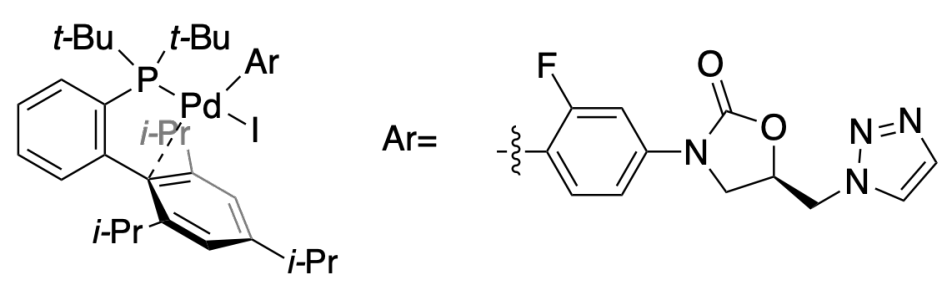

0
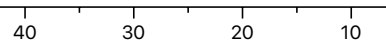

$-10$ $-20$

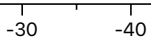
-50
$\mathrm{f} 1(\mathrm{ppm})$ 
(t-BuXPhos)Pd(X14)I (S10)

${ }^{31} \mathrm{P}$ NMR (202 MHz, $\mathrm{CD}_{2} \mathrm{Cl}_{2}$ )
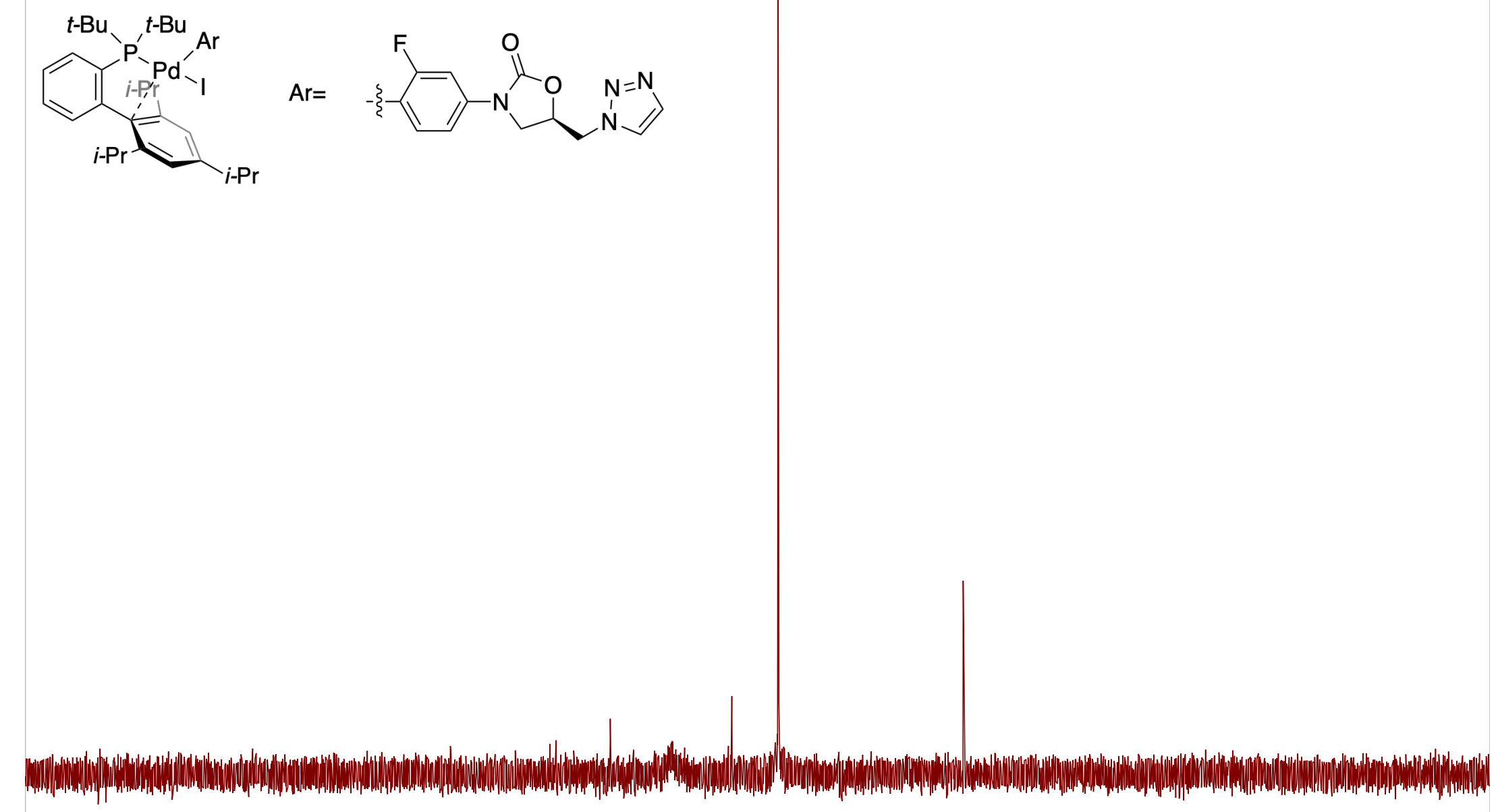

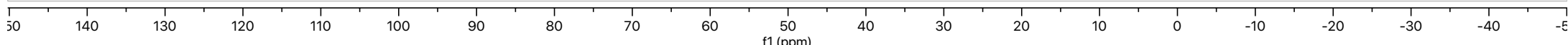




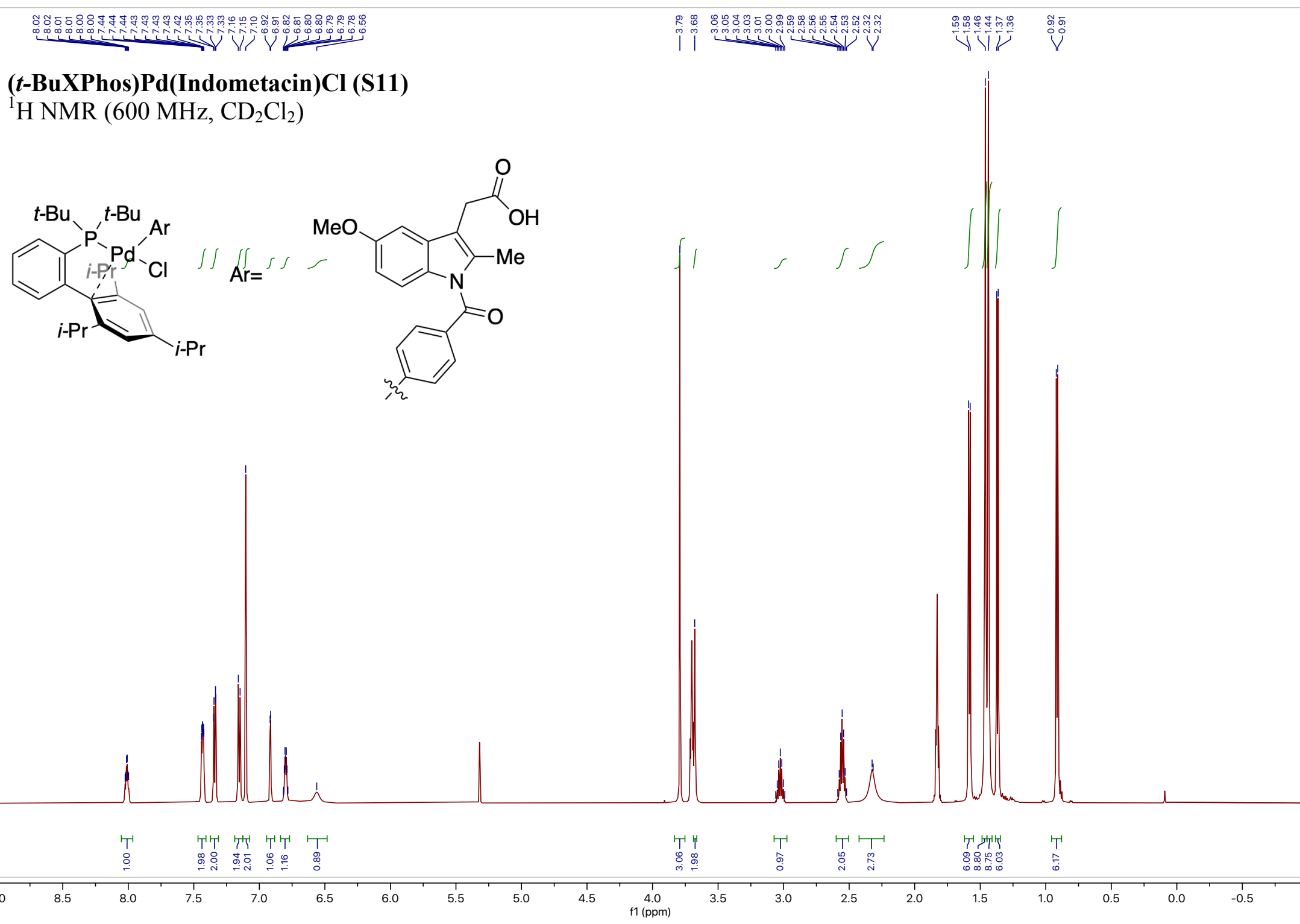




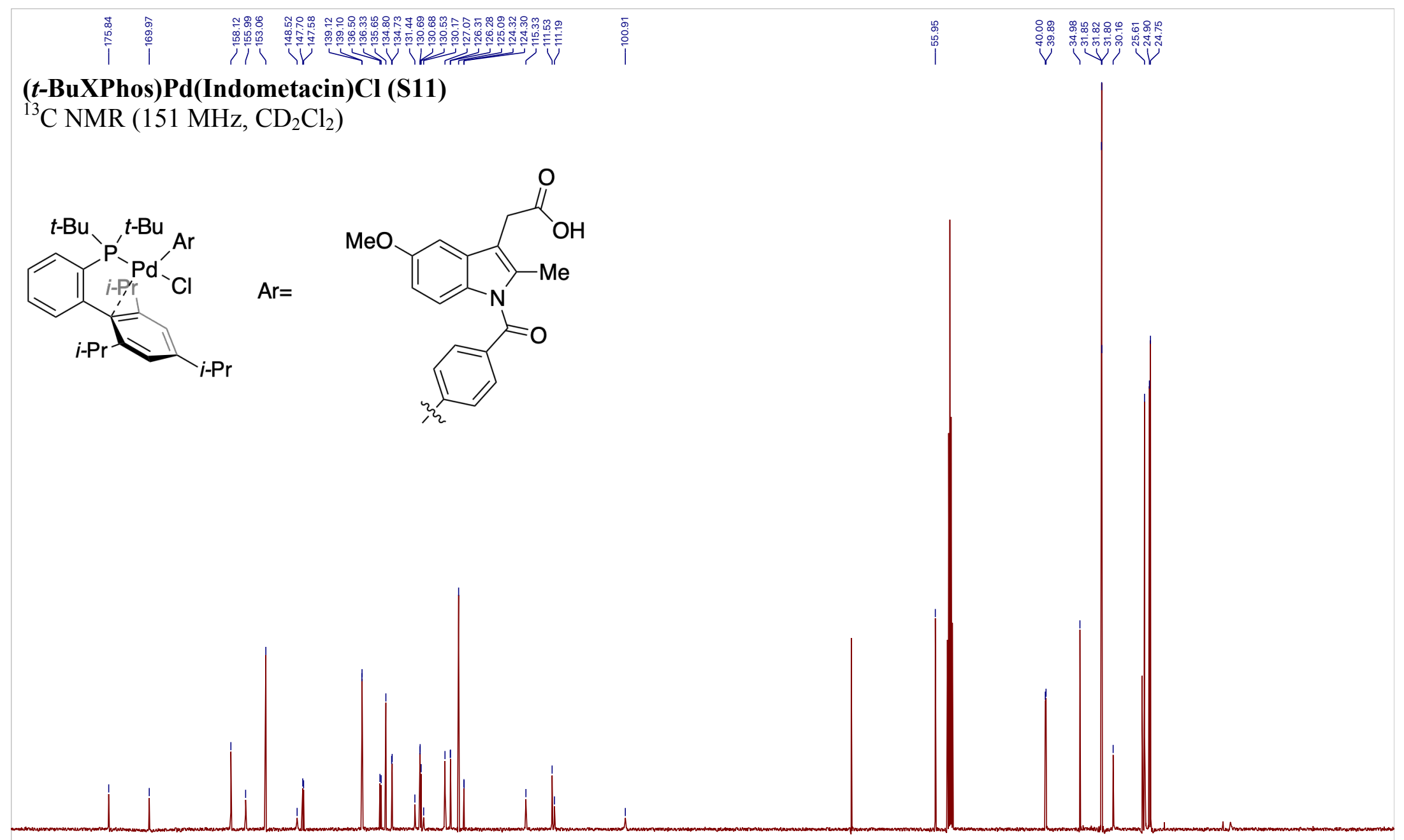


(t-BuXPhos)Pd(Indometacin)Cl (S11)

${ }^{31} \mathrm{P}$ NMR (202 MHz, $\mathrm{CD}_{2} \mathrm{Cl}_{2}$ )

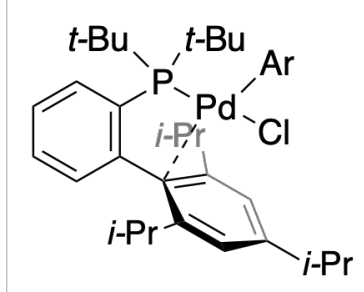

$\operatorname{Ar}=$

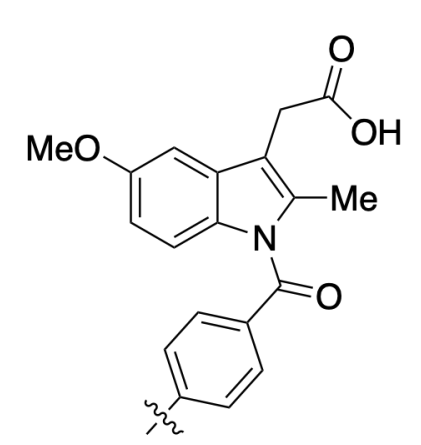

140 130 120 110 100 90 80 
(t-BuBrettPhos)Pd(rivaroxaban)Cl (S12)

${ }^{1} \mathrm{H}$ NMR $\left(600 \mathrm{MHz}, \mathrm{CD}_{2} \mathrm{Cl}_{2}\right.$ )
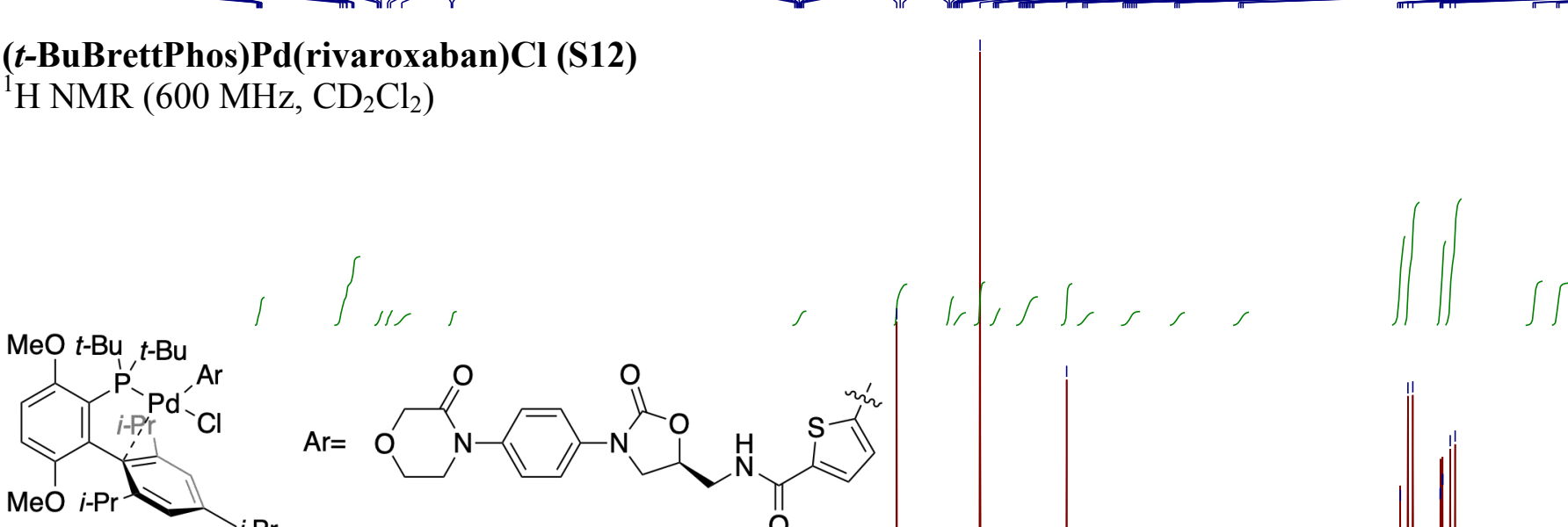

$\operatorname{Ar}=$
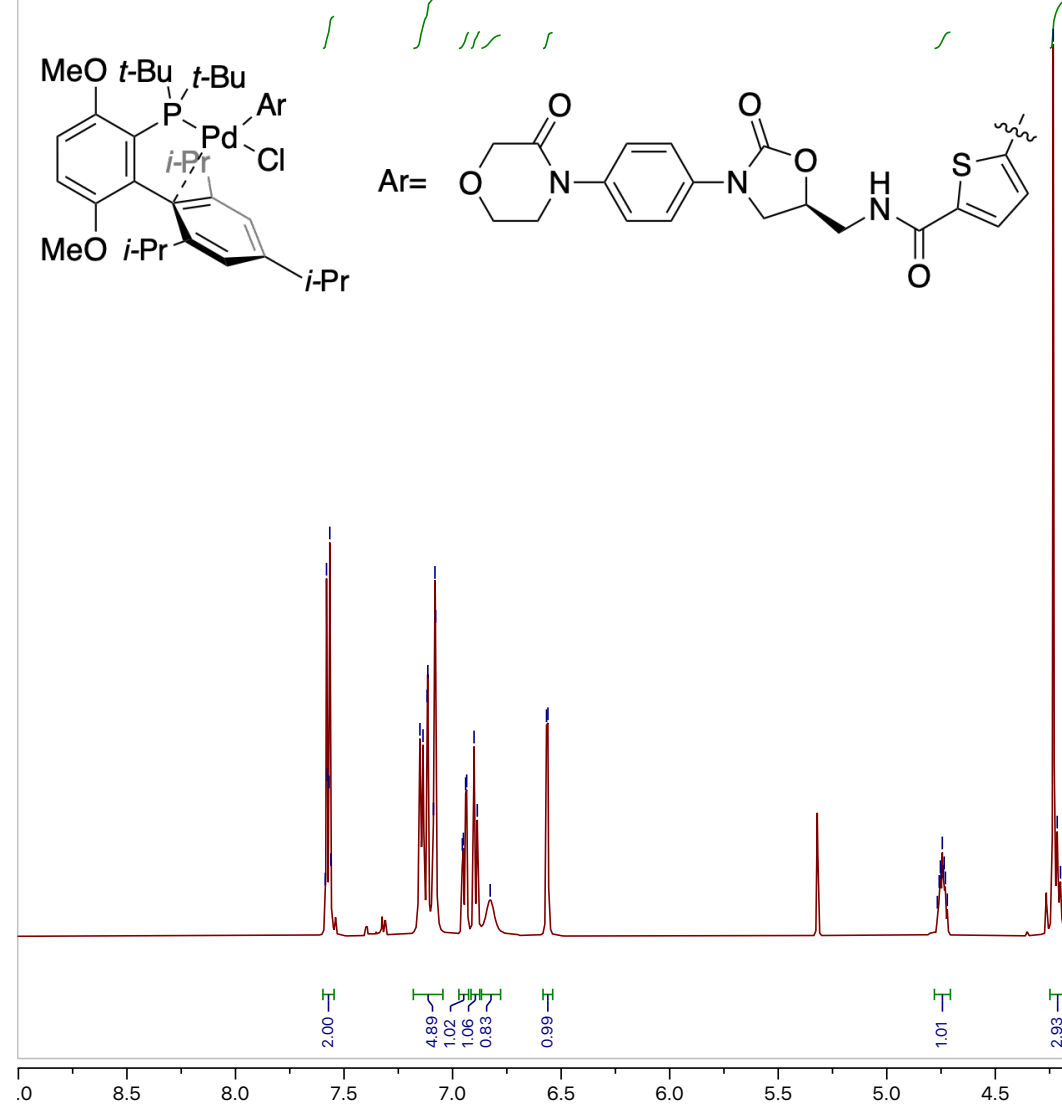

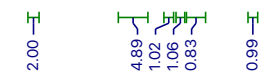
6.5 6.0 4.5 (ppm) 


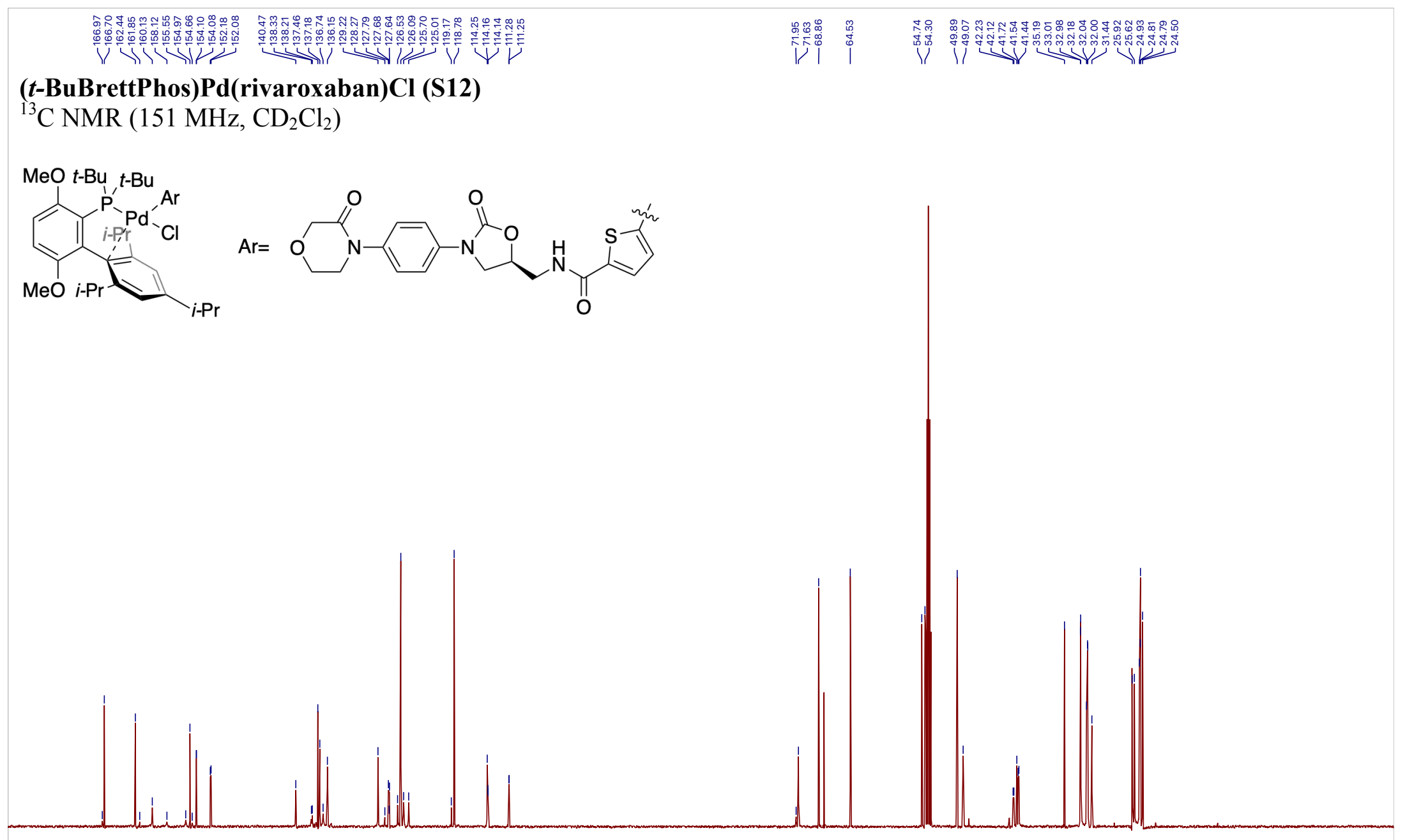




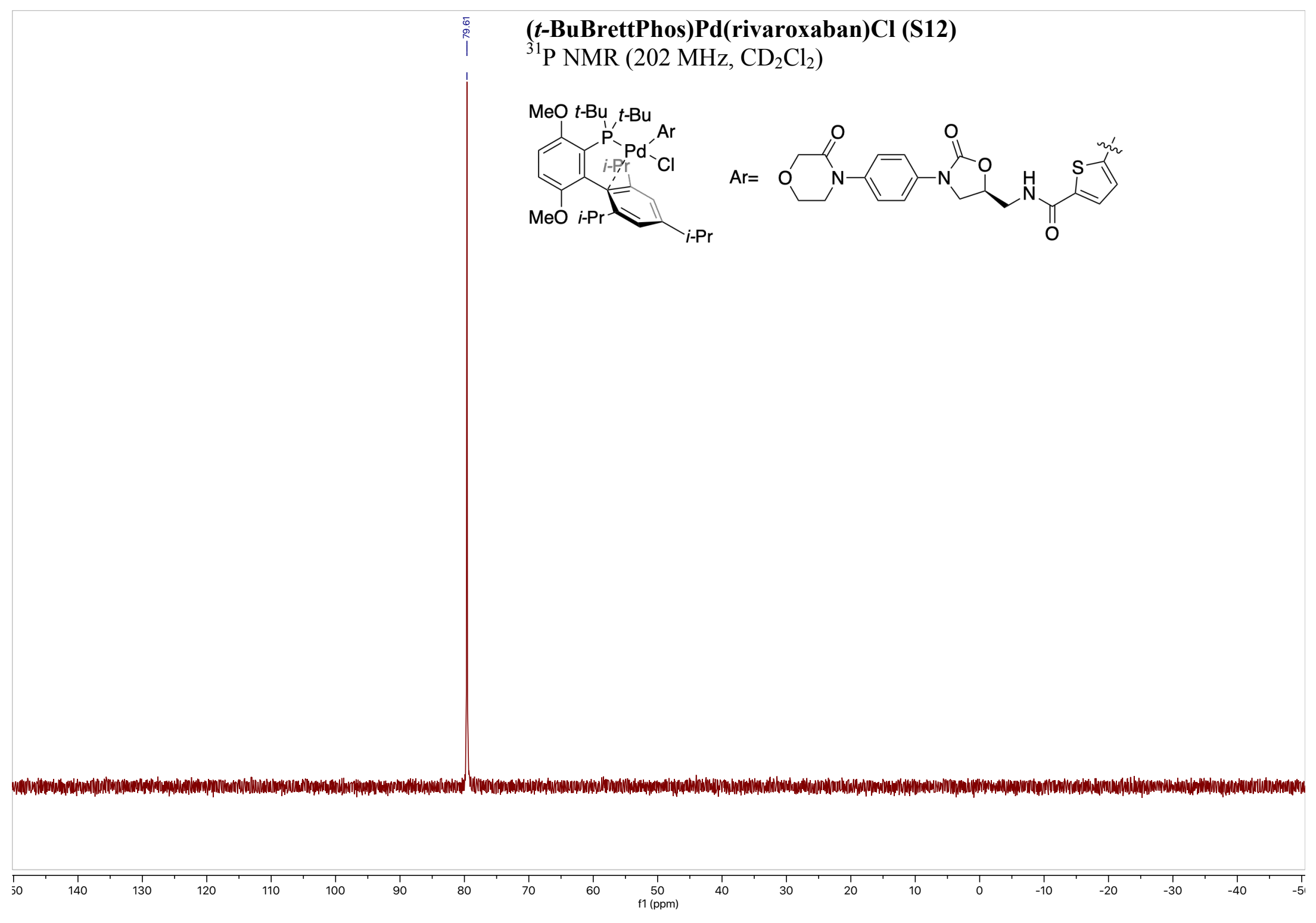




\section{(XPhos)Pd(rivaroxaban)Cl (2a)}

${ }^{1} \mathrm{H}$ NMR $\left(500 \mathrm{MHz}, \mathrm{CD}_{2} \mathrm{Cl}_{2}\right.$ )

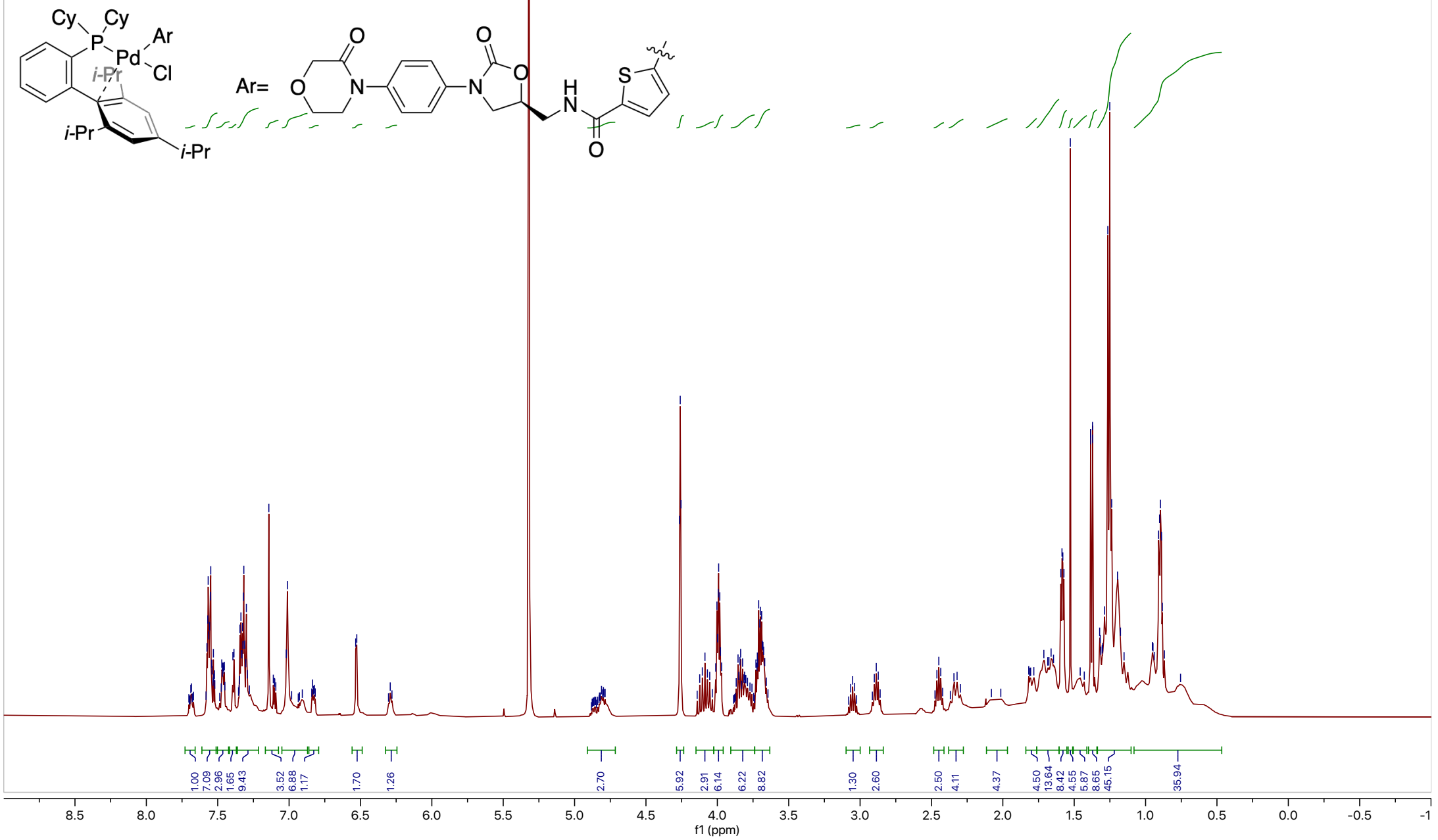




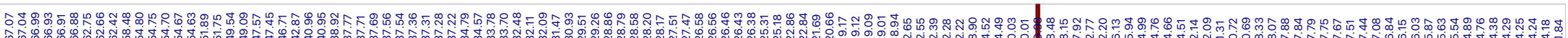

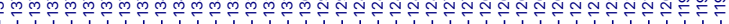
(XPhos)Pd(rivaroxaban)Cl (2a)

${ }^{13} \mathrm{C} \mathrm{NMR}\left(151 \mathrm{MHz}, \mathrm{CD}_{2} \mathrm{Cl}_{2}\right)$

(n)

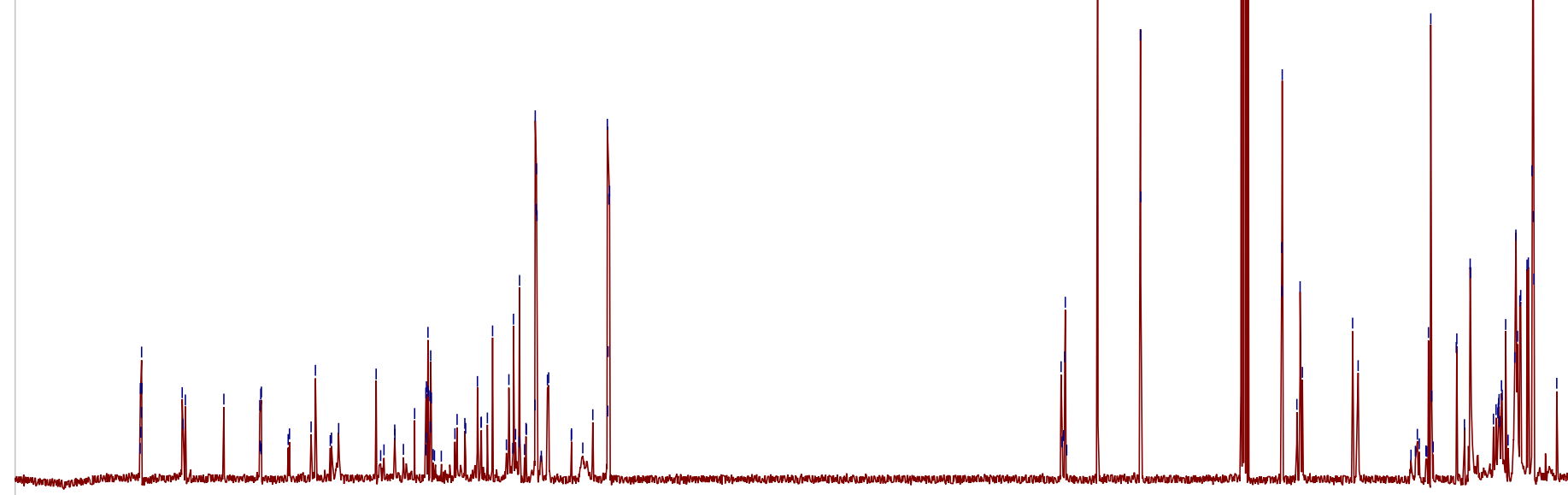


(XPhos)Pd(rivaroxaban)Cl (2a)

${ }^{31} \mathrm{P}$ NMR (203 MHz, $\mathrm{CD}_{2} \mathrm{Cl}_{2}$ )

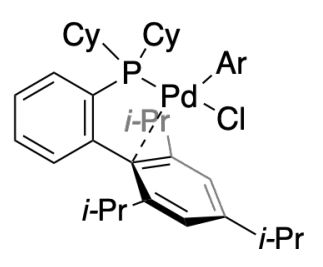

$\mathrm{Ar}=$

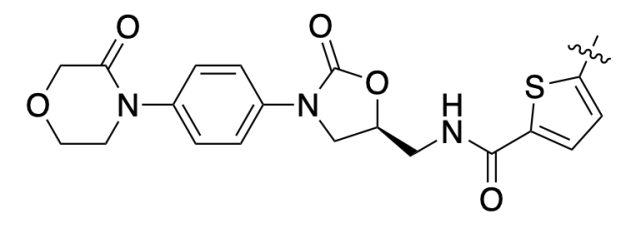

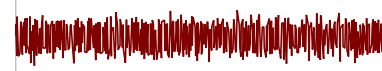

50

$140 \quad 130 \quad 120$ 110 100 $90 \quad 80$ 70,60 $\underset{f 1(p p m)}{50}$ 40 30 20 10 0 $-10$ 


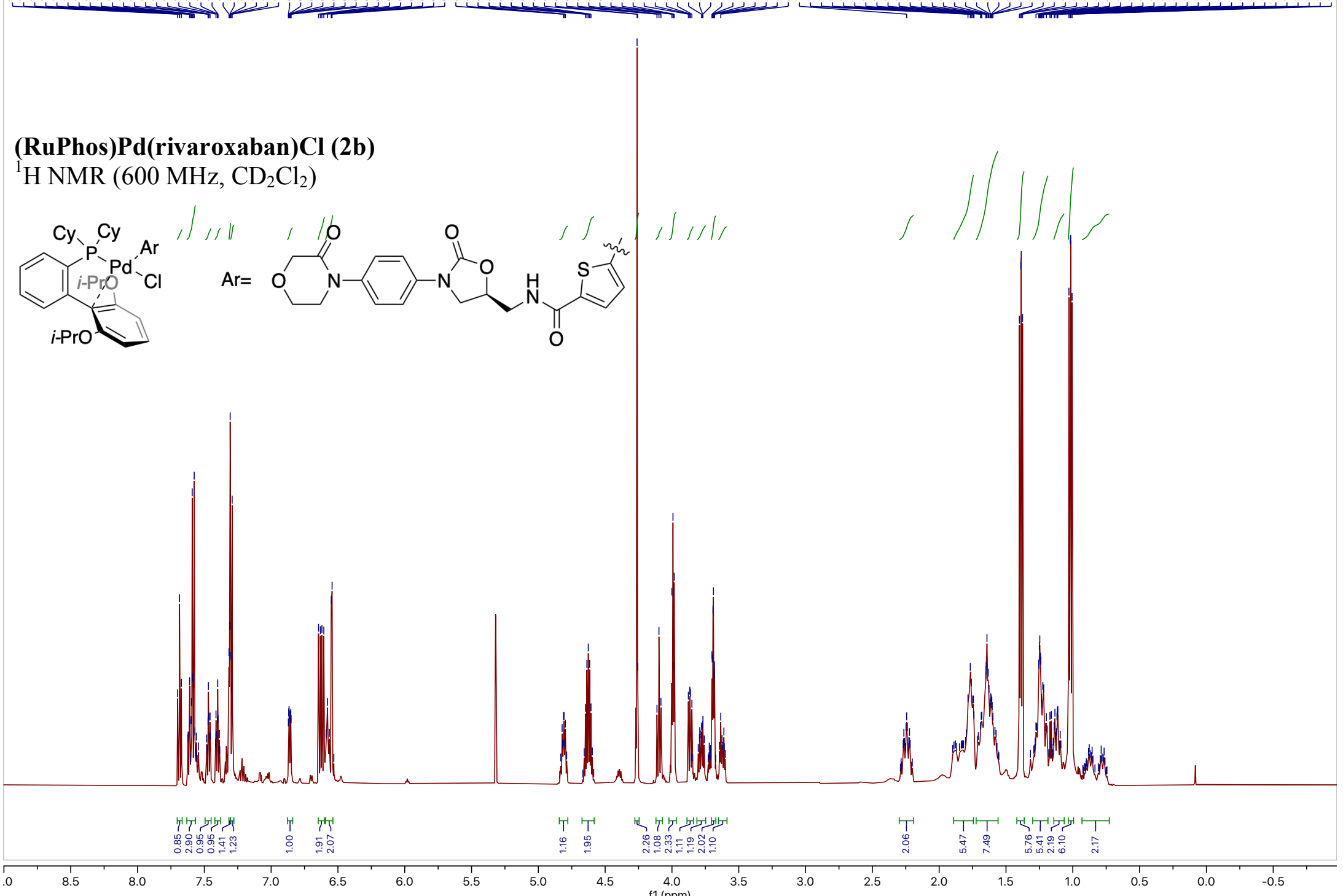




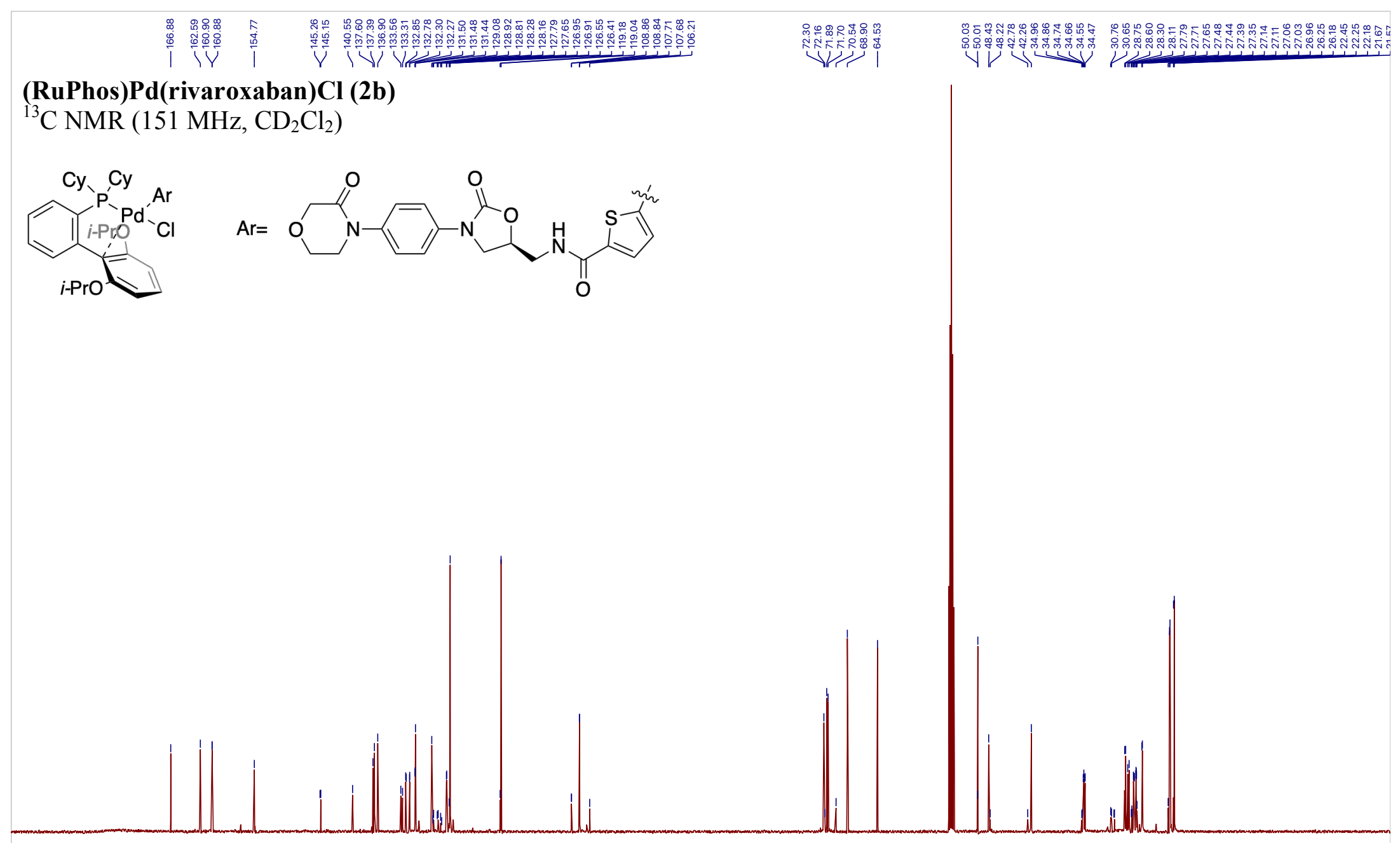


(RuPhos)Pd(rivaroxaban)Cl (2b)

${ }^{31} \mathrm{P}$ NMR (203 MHz, $\mathrm{CD}_{2} \mathrm{Cl}_{2}$ )

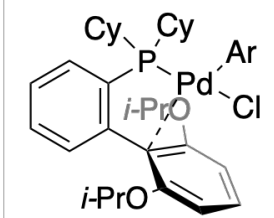

$\mathrm{Ar}=$

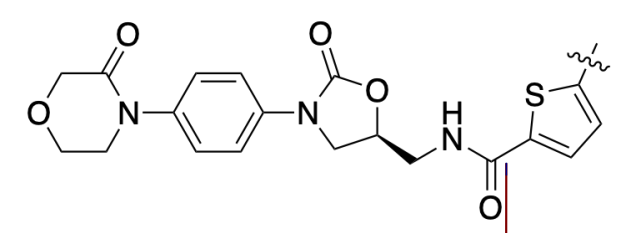

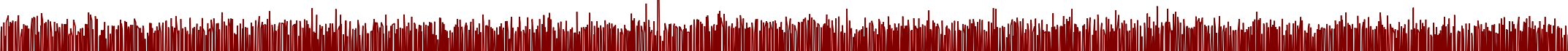

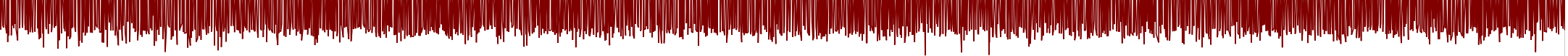

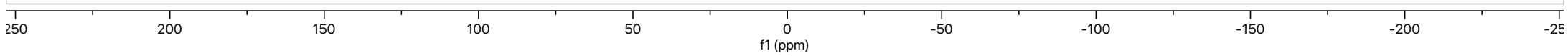




\section{(SPhos)Pd(rivaroxaban)Cl (2c)}

${ }^{1} \mathrm{H}$ NMR (600 MHz, $\mathrm{CD}_{2} \mathrm{Cl}_{2}$ )

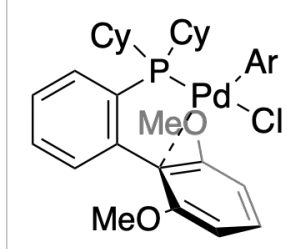

$\operatorname{Ar}=$

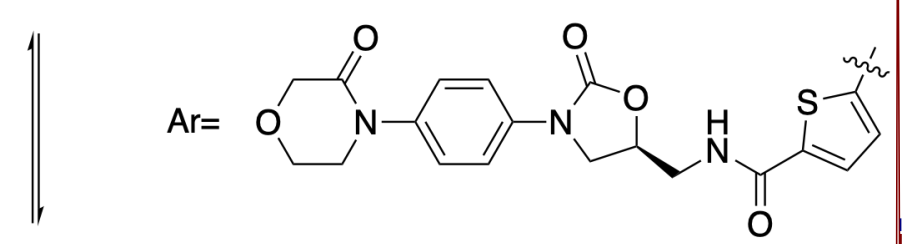

$\mathrm{Ar}^{\mathrm{L}}{ }_{\mathrm{Pd}}^{\mathrm{Cl}} \mathrm{Cl}_{\mathrm{Pd}}^{\mathrm{Cl}}$ N W
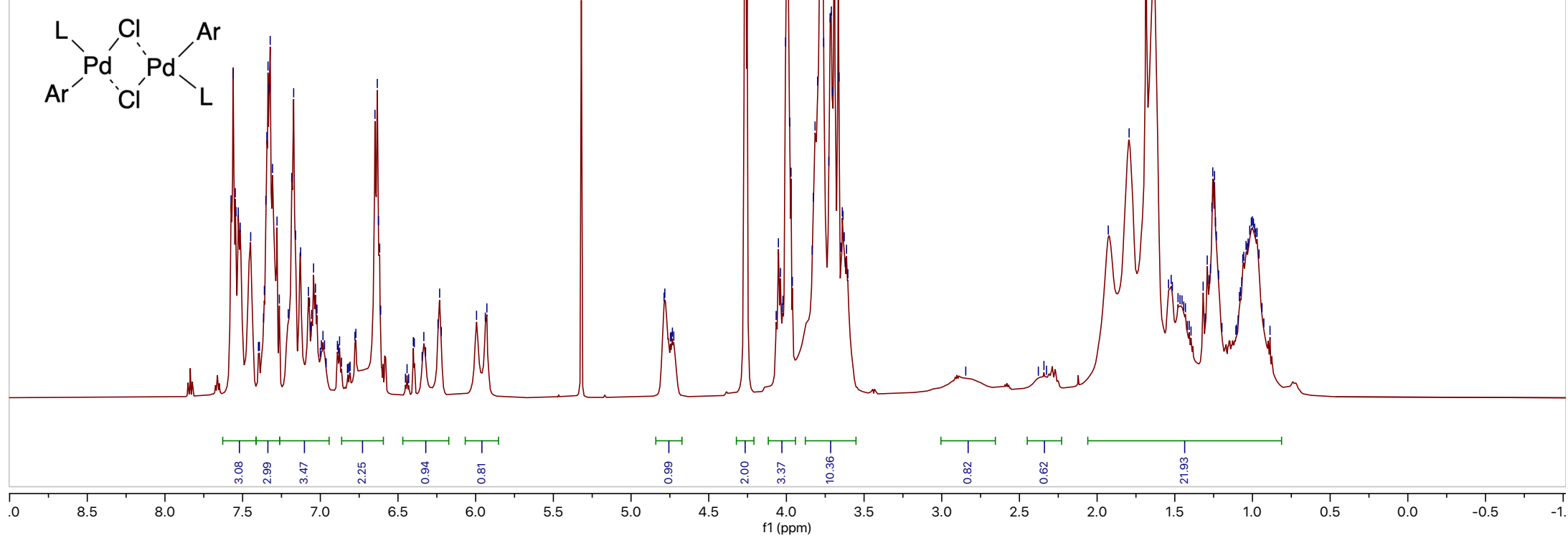


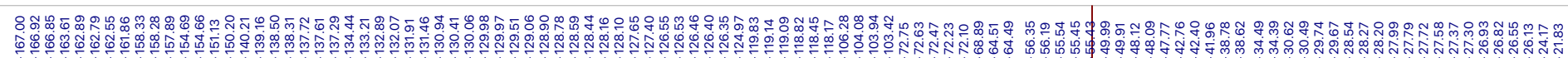

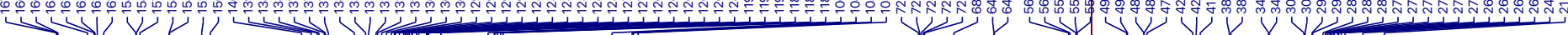

(SPhos)Pd(rivaroxaban)Cl (2c)

${ }^{13} \mathrm{C}$ NMR (151 MHz, $\mathrm{CD}_{2} \mathrm{Cl}_{2}$ )

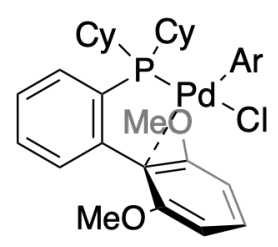

$\operatorname{Ar}=$

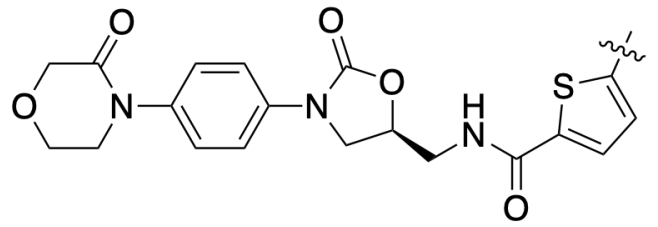

$\mathrm{Ar}^{\mathrm{L}}{ }^{\mathrm{Pd}}{ }_{\mathrm{CCl}}^{\mathrm{Cl}} \mathrm{Pd}^{\prime} \mathrm{Pr}$

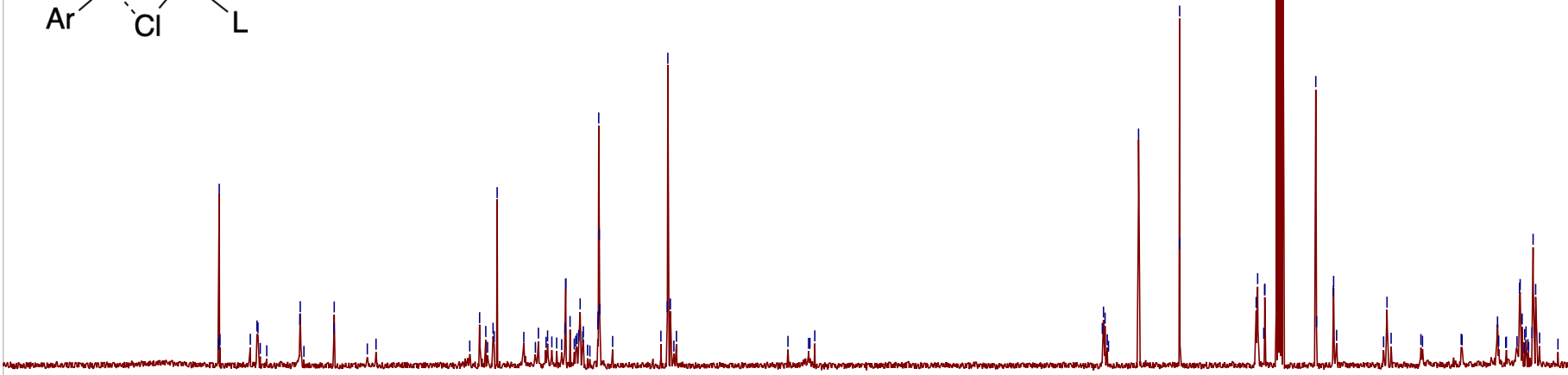


(SPhos)Pd(rivaroxaban)Cl (2c)

${ }^{31}$ P NMR (203 MHz, $\mathrm{CD}_{2} \mathrm{Cl}_{2}$ ): Concentrated

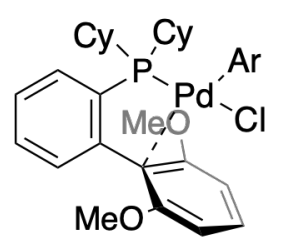

$\operatorname{Ar}=$

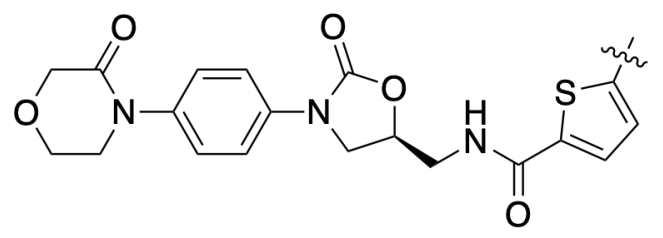

$\mathrm{L}, \mathrm{Cl}$

$\mathrm{Ar}^{\prime} \mathrm{Pd}$ "CI $\mathrm{Cl} \mathrm{Pd}^{\prime}$

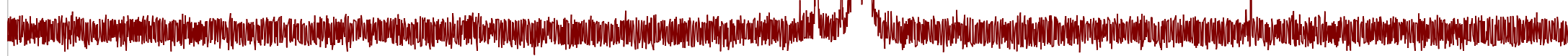

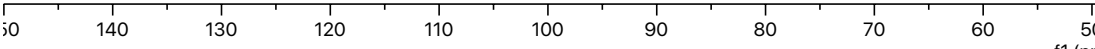

$1(\mathrm{ppm})$


(SPhos)Pd(rivaroxaban)Cl (2c)

${ }^{31} \mathrm{P}$ NMR $\left(203 \mathrm{MHz}, \mathrm{CD}_{2} \mathrm{Cl}_{2}\right)$ : Dilute
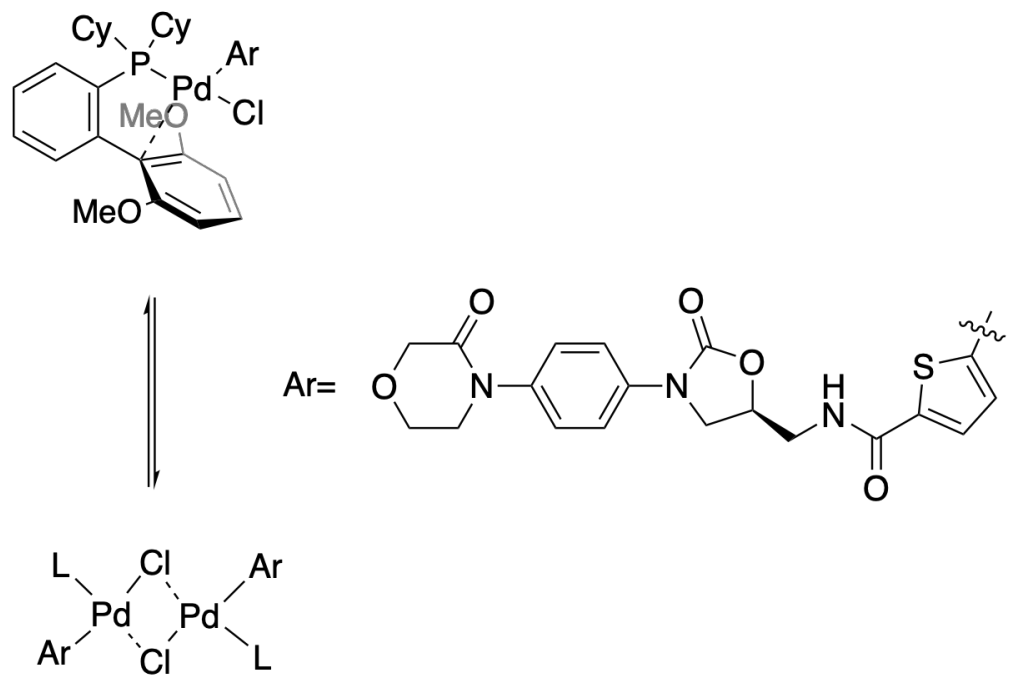

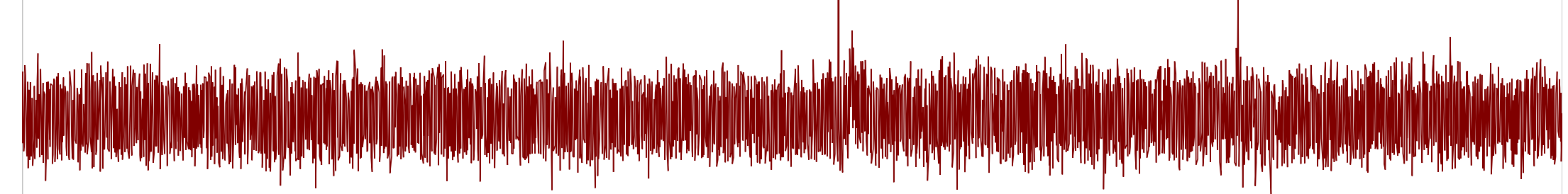

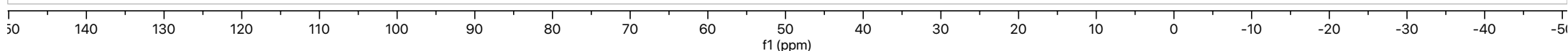




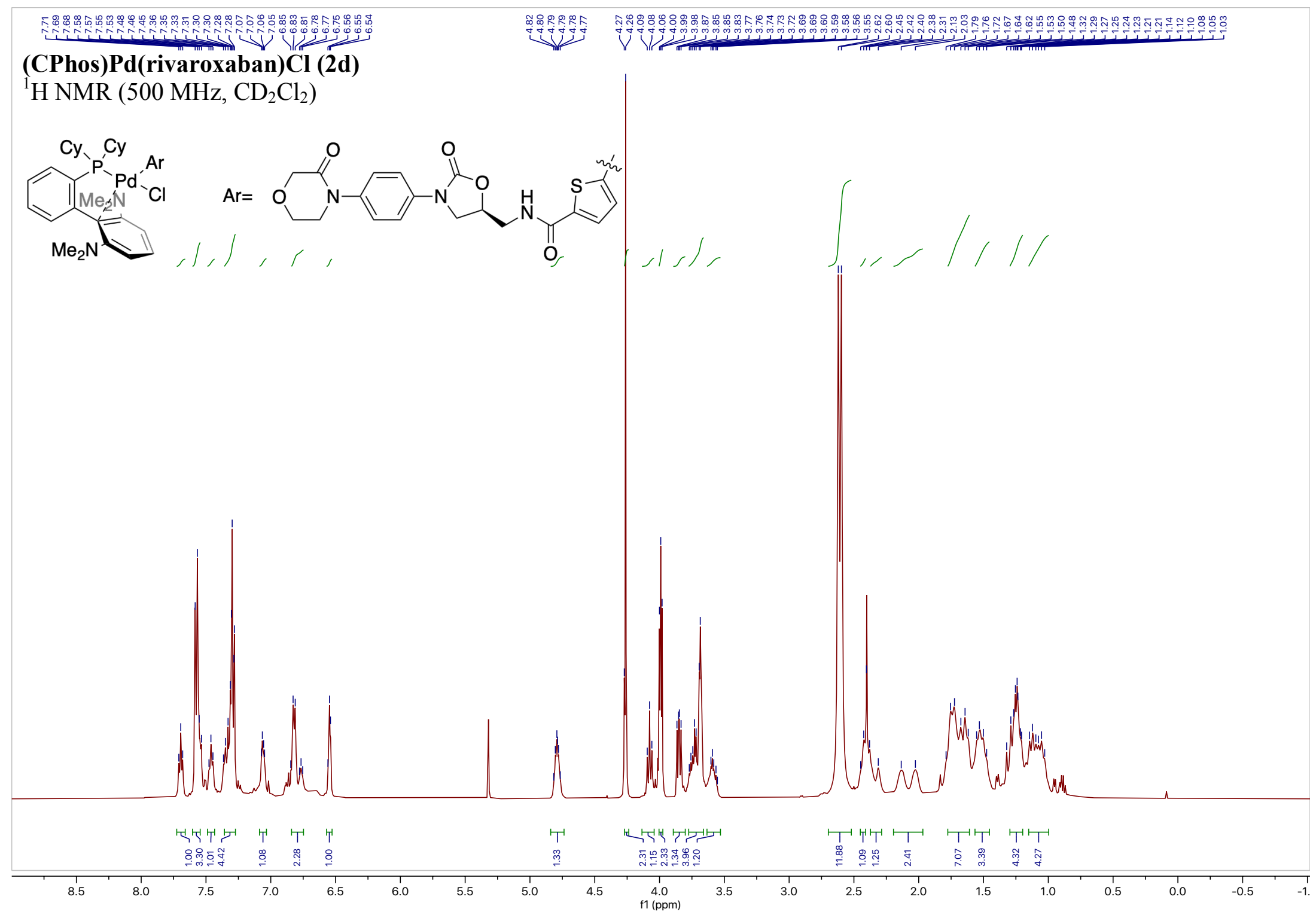




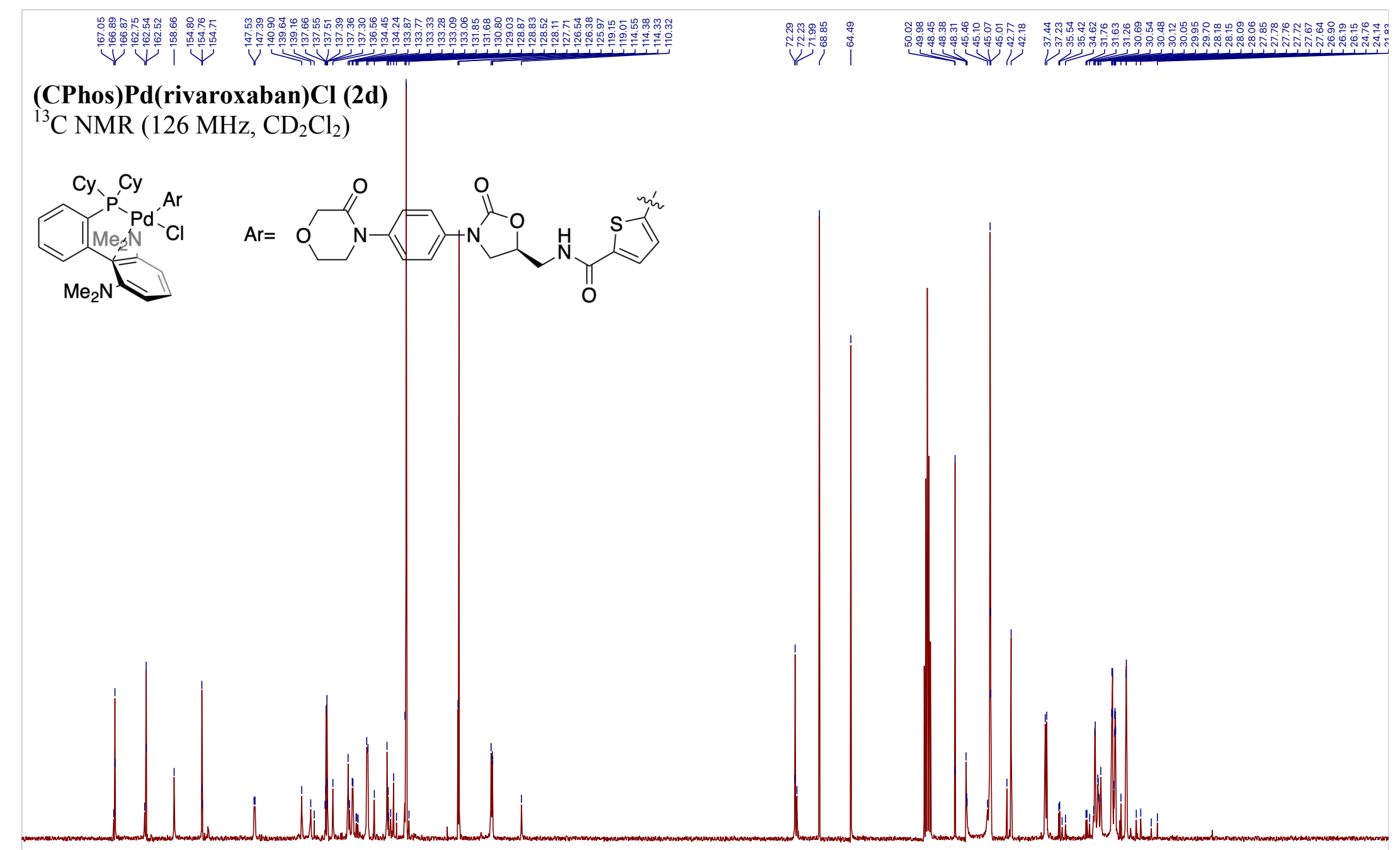


(CPhos)Pd(rivaroxaban)Cl (2d)

${ }^{31} \mathrm{P}$ NMR (203 MHz, $\mathrm{CD}_{2} \mathrm{Cl}_{2}$ )
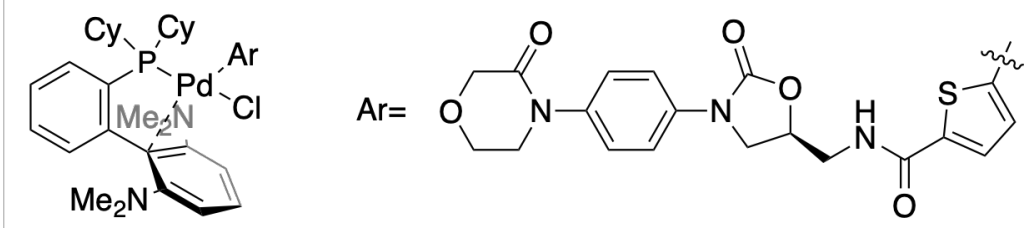

$\mathrm{O}$

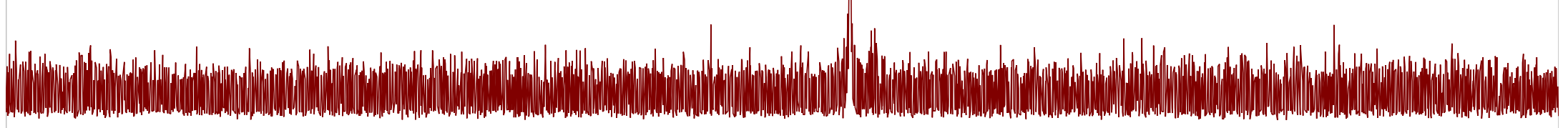


(PhXPhos)Pd(rivaroxaban)Cl (2e)

${ }^{1} \mathrm{H}$ NMR $\left(500 \mathrm{MHz}, \mathrm{CD}_{2} \mathrm{Cl}_{2}\right.$ )

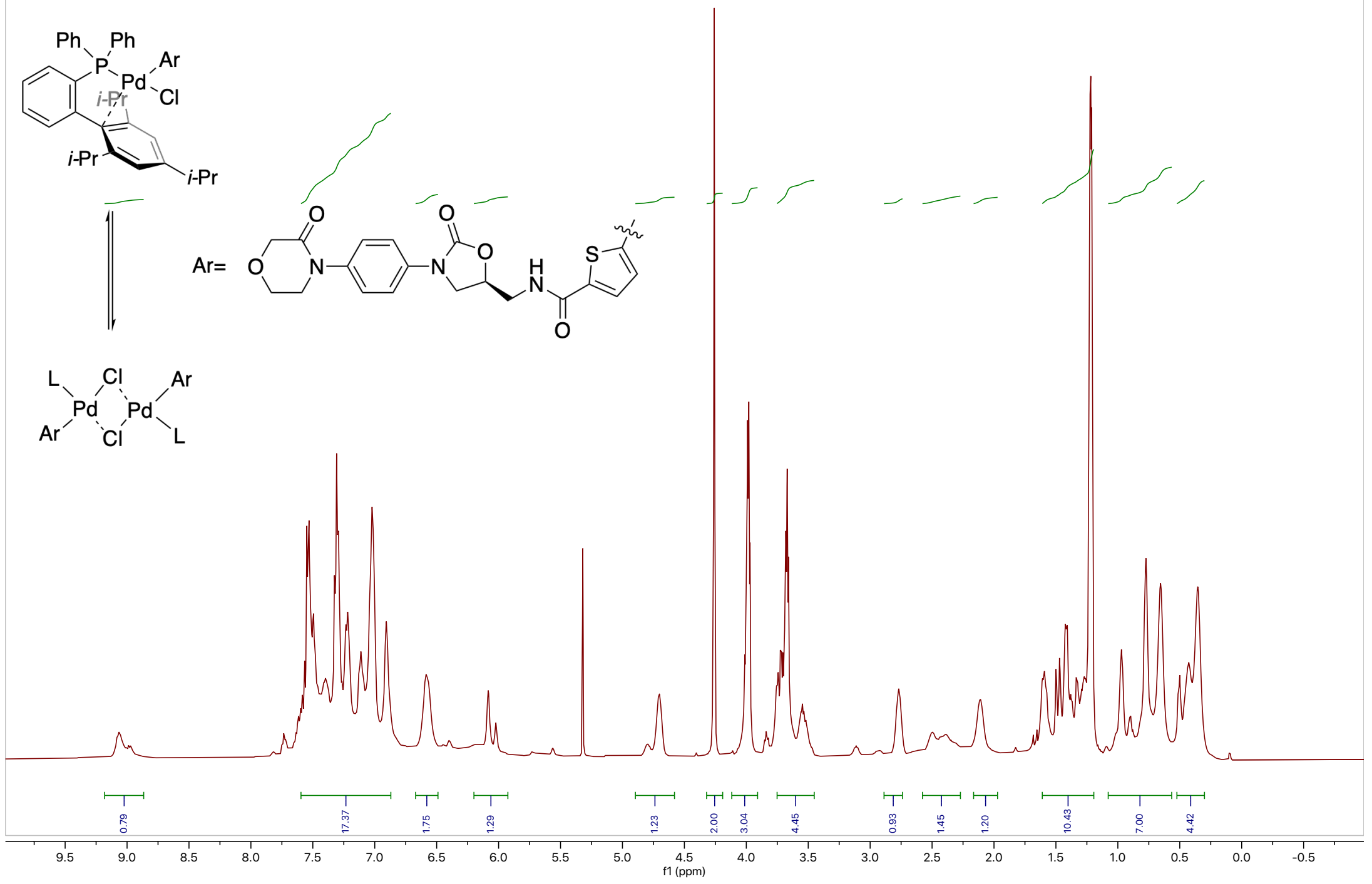




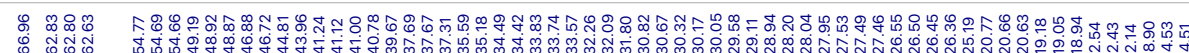

\begin{tabular}{lll} 
& \\
\hline
\end{tabular}

(PhXPhos)Pd(rivaroxaban)Cl (2e)

${ }^{13} \mathrm{C}$ NMR $\left(126 \mathrm{MHz}, \mathrm{CD}_{2} \mathrm{Cl}_{2}\right)$
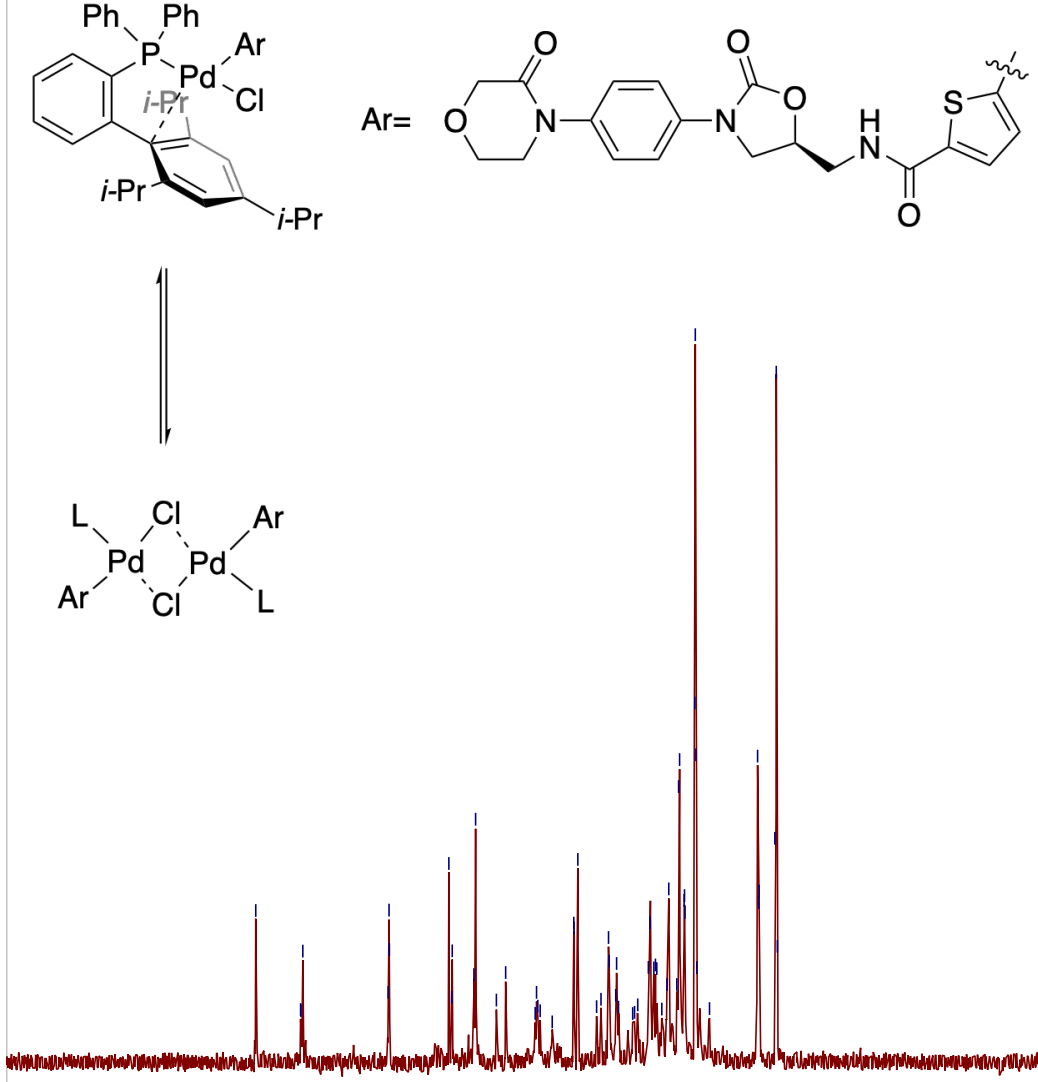

$180 \quad 170$

160

$150 \quad 140$

130

120

110

$100 \quad 90$

80

70

60

50

40

30

20 
(PhXPhos)Pd(rivaroxaban)Cl (2e)

${ }^{31}$ P NMR (203 MHz, $\mathrm{CD}_{2} \mathrm{Cl}_{2}$ ): Concentrated

$\mathrm{Ph} 、 \mathrm{Ph}$

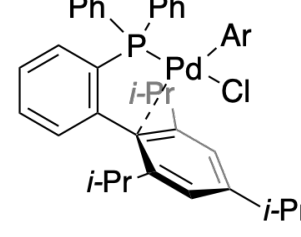

$\operatorname{Ar}=$<smiles>Cc1ccc(C(=O)NCC2CN(c3ccc(N4CCOCC4=O)cc3)C(=O)O2)s1</smiles>

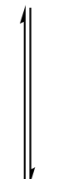

$\mathrm{Ar}^{\mathrm{L}} \mathrm{Pd}^{\mathrm{Cl}} \mathrm{CI}^{\mathrm{Cl}} \mathrm{Pd}$

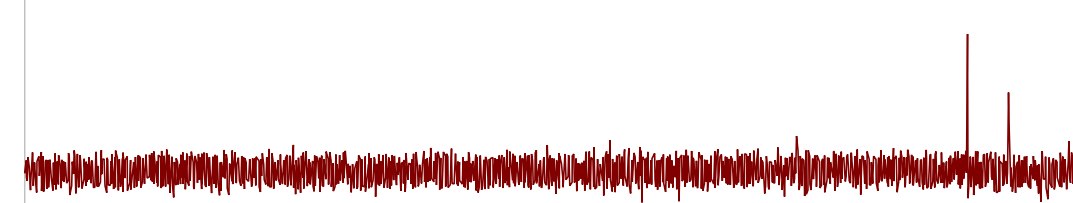

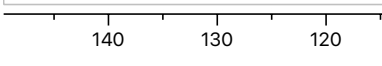

110

100

90

$80+70$

60 50
$f 1(p p m)$ 30 20 10 $-10$ $-20$ 
(PhXPhos)Pd(rivaroxaban)Cl (2e)

${ }^{31} \mathrm{P}$ NMR (203 MHz, $\mathrm{CD}_{2} \mathrm{Cl}_{2}$ ): Dilute

ind

$\operatorname{Ar}=$
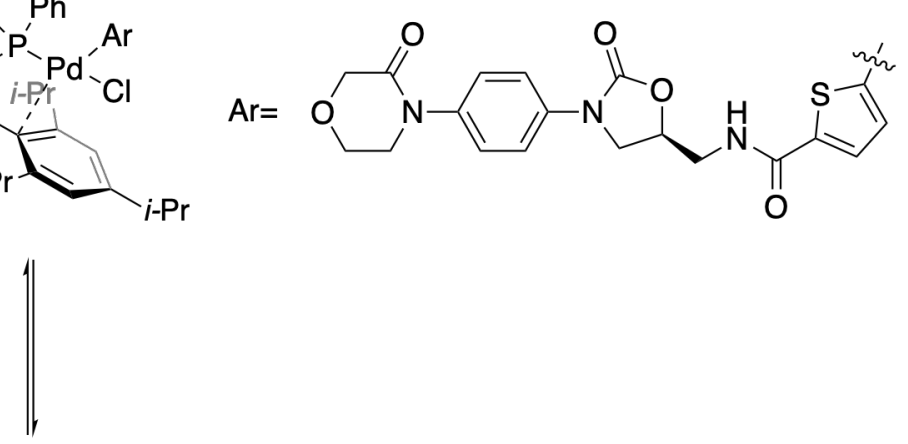

$\mathrm{Ar}^{\mathrm{L}} \mathrm{Pd}_{\mathrm{Cl}}^{\mathrm{Cl}} \mathrm{Pd} \mathrm{Pd}_{\mathrm{L}}^{\mathrm{Ar}}$

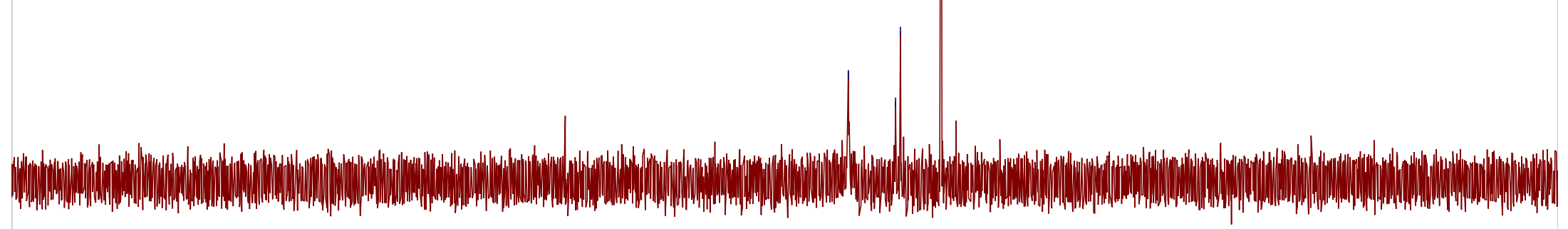




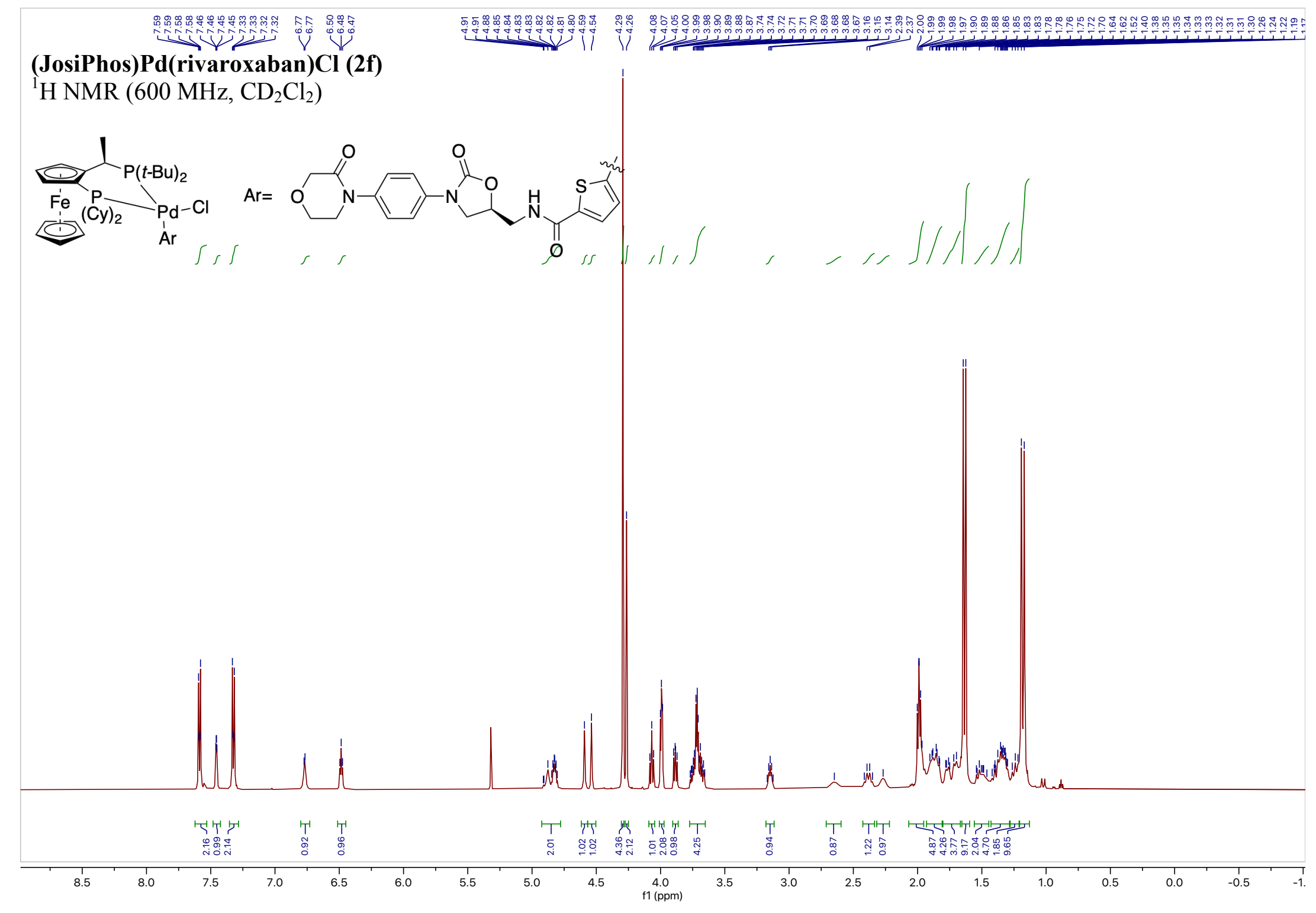




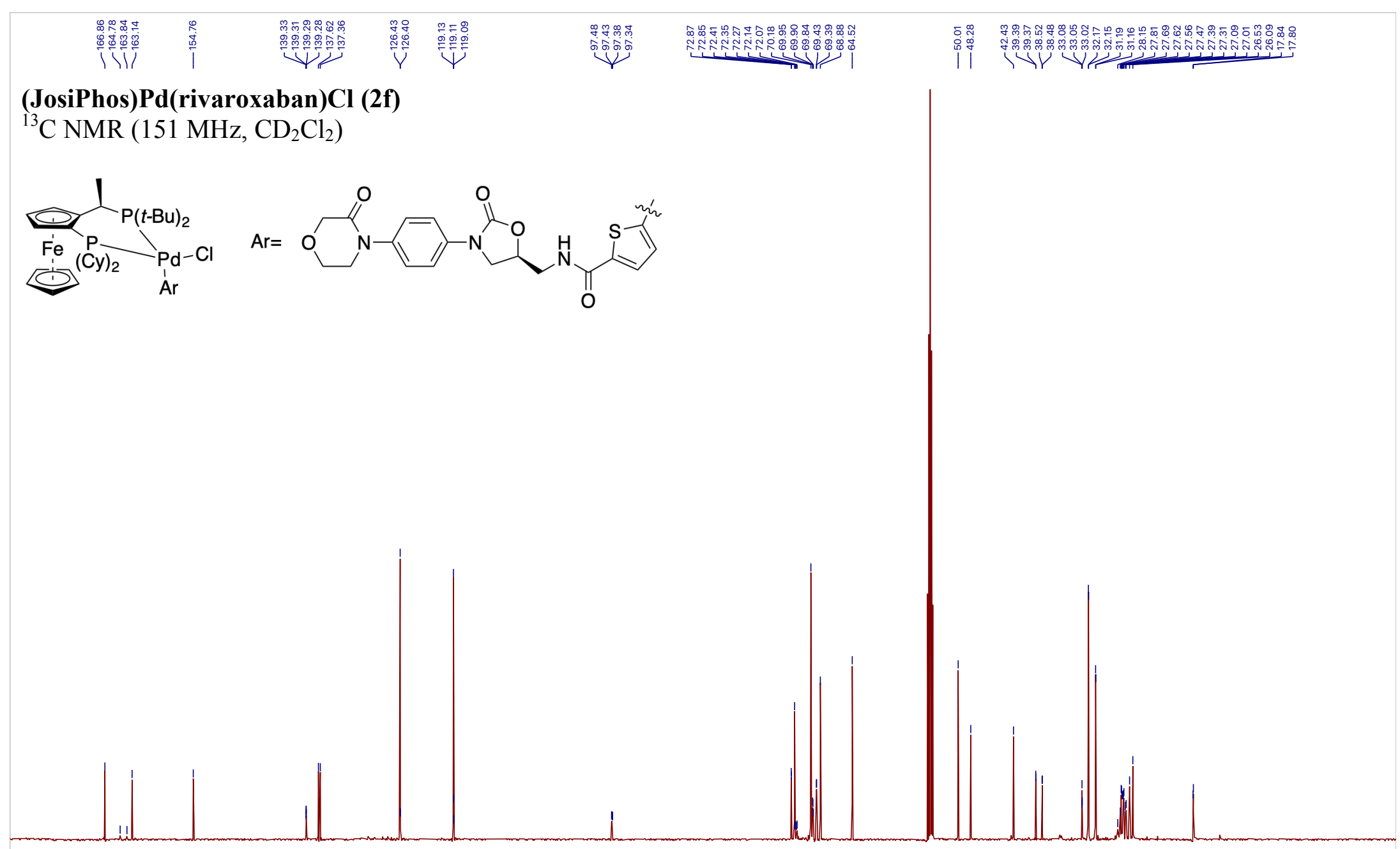

170

160 150 140 130 120 110 100 $90 \mathrm{f1}(\mathrm{ppm})$ 60

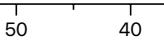
30 20 


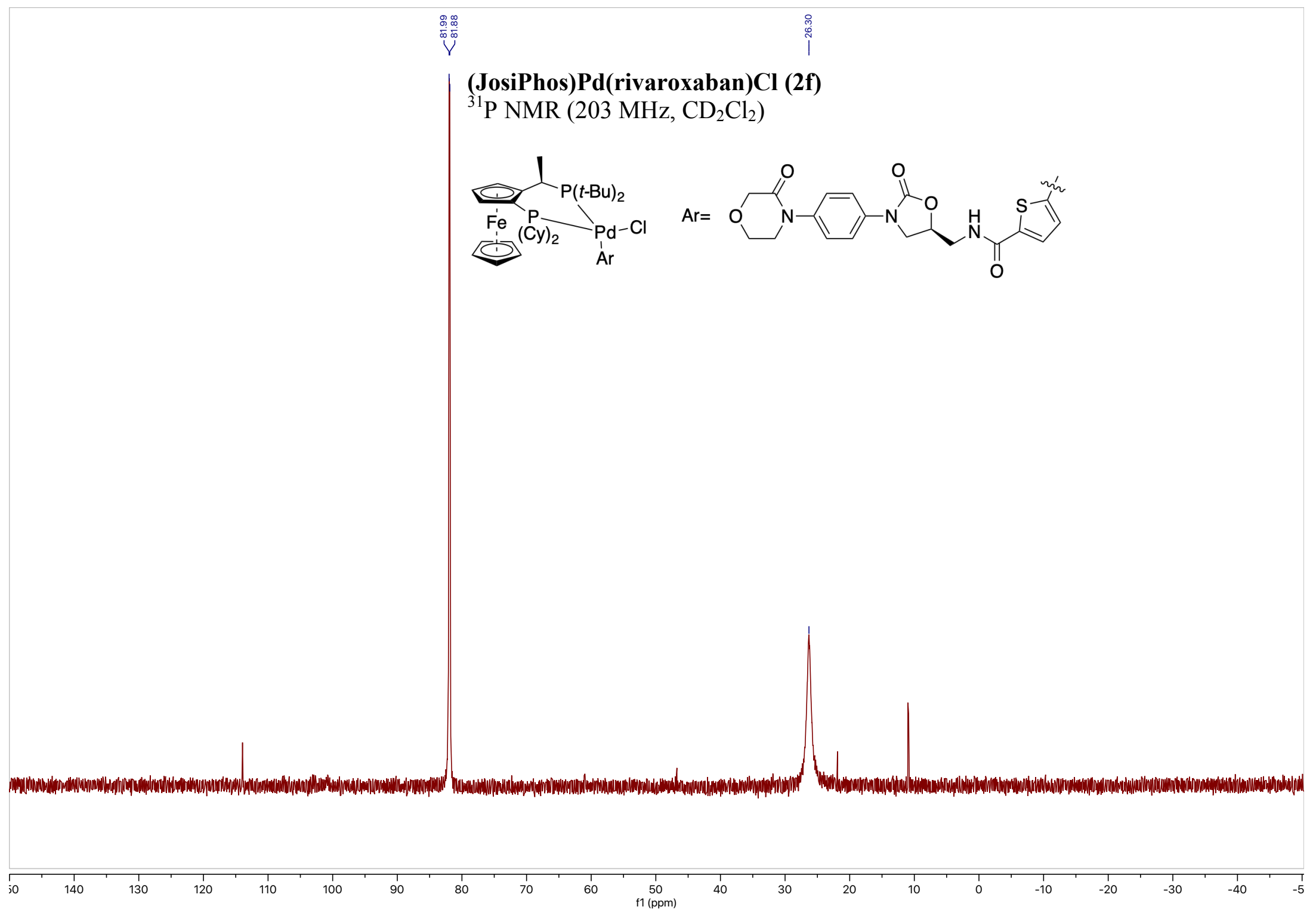




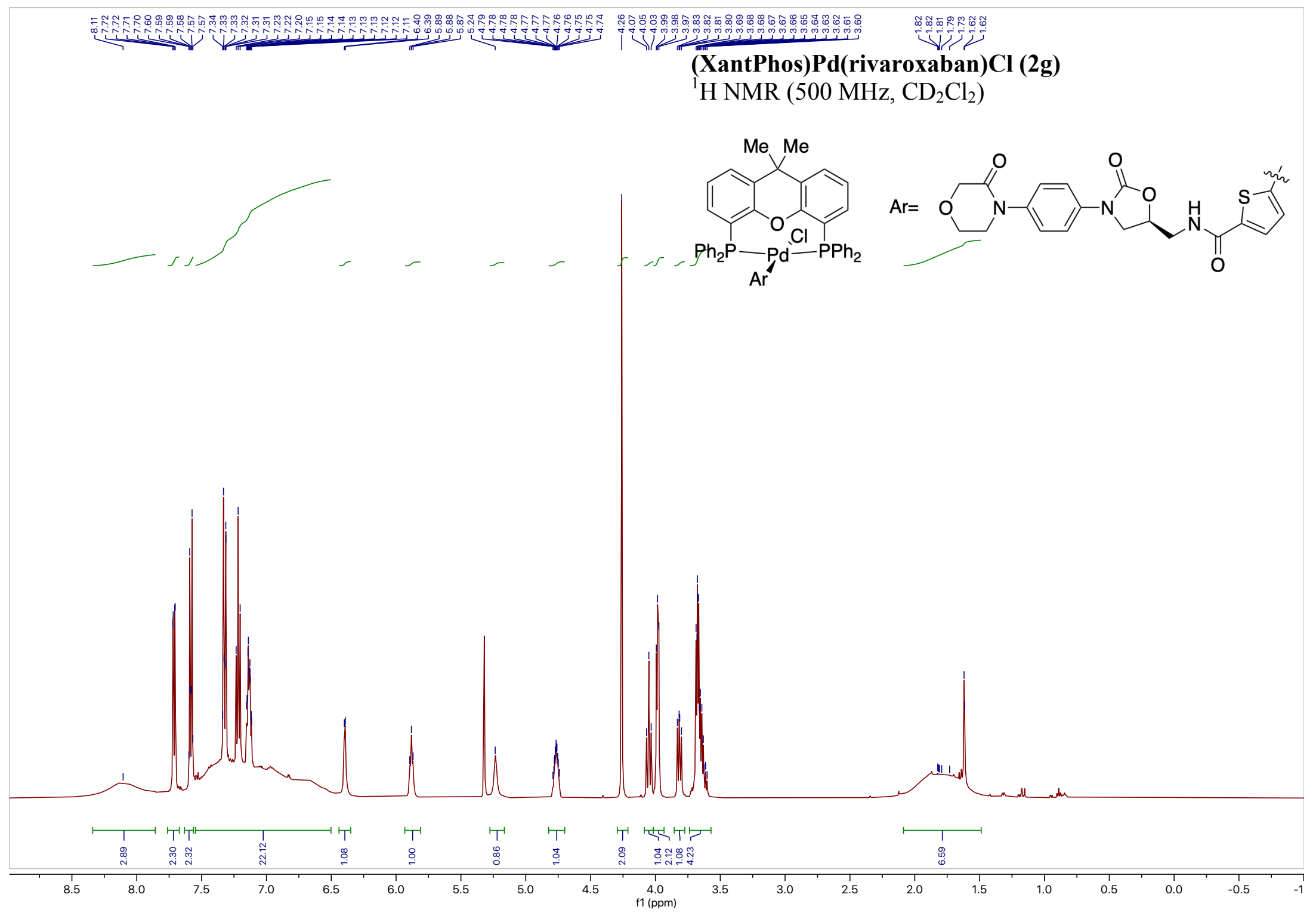




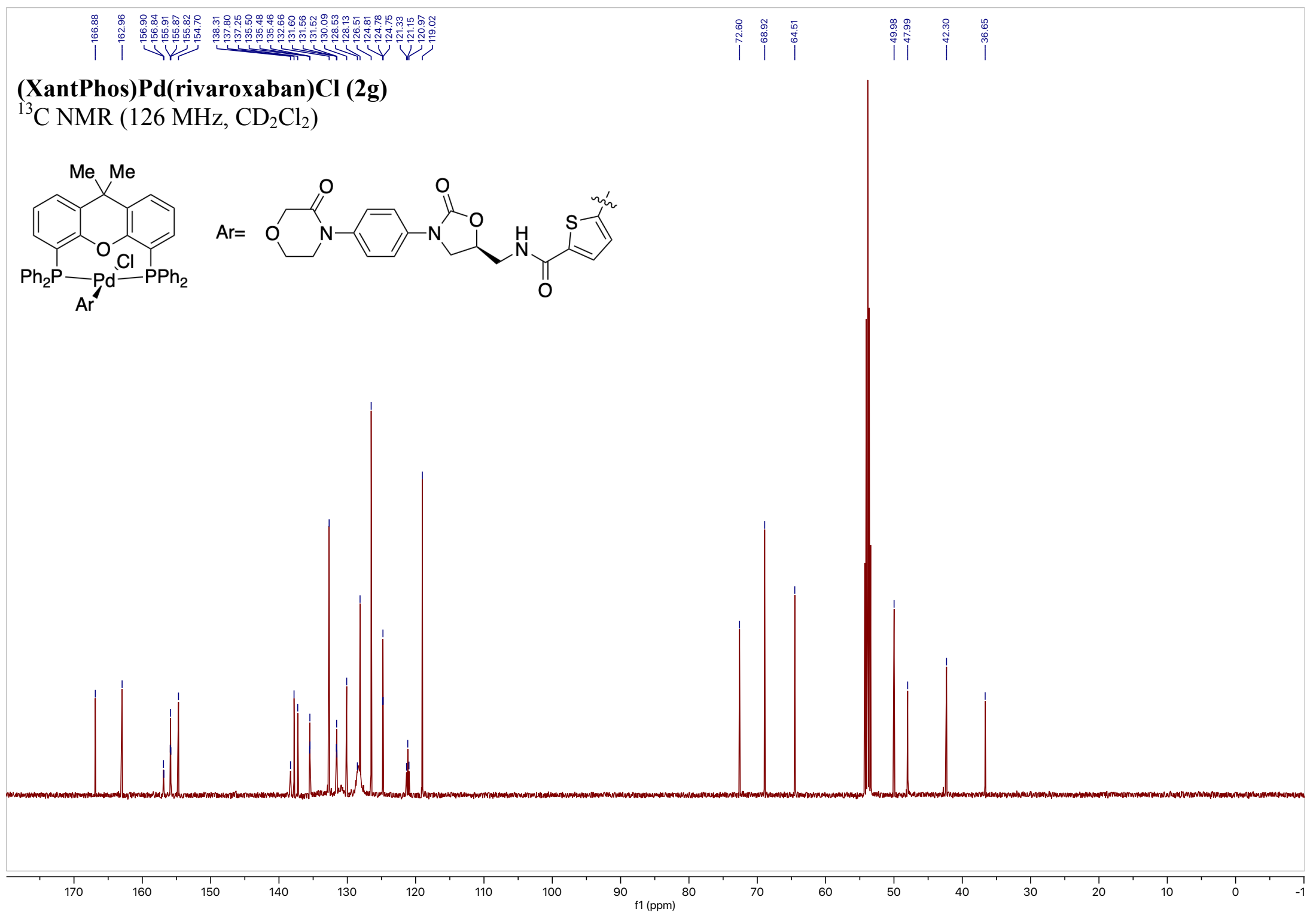

S109 
(XantPhos)Pd(rivaroxaban)Cl (2g)

${ }^{31} \mathrm{P}$ NMR (203 MHz, $\mathrm{CD}_{2} \mathrm{Cl}_{2}$ )
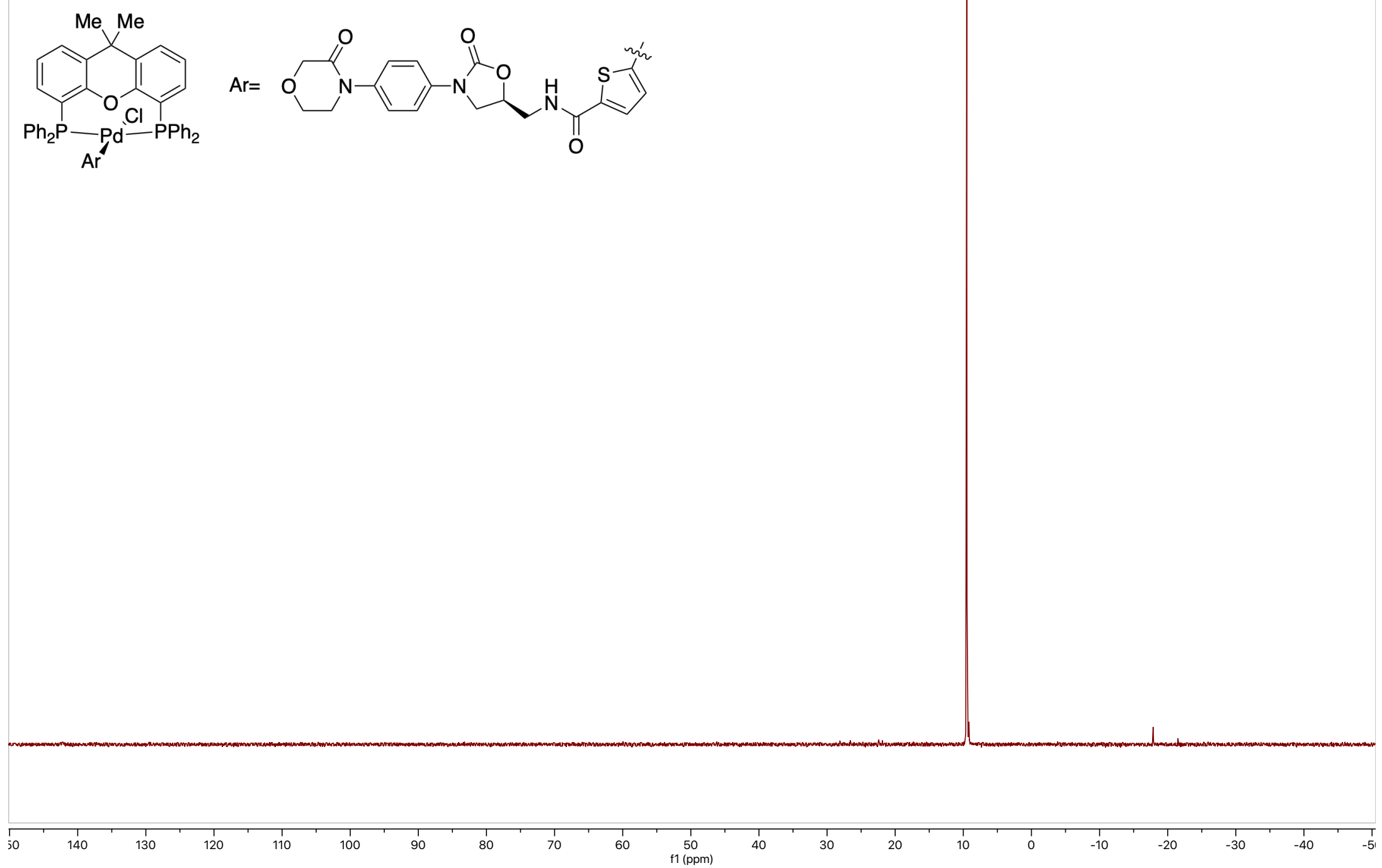


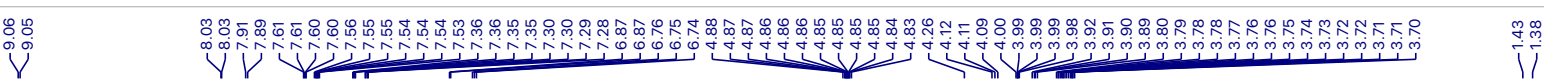

(dtbbpy)Pd(rivaroxaban)Cl (2h)

${ }^{1} \mathrm{H}$ NMR $\left(600 \mathrm{MHz}, \mathrm{CD}_{2} \mathrm{Cl}_{2}\right.$ )
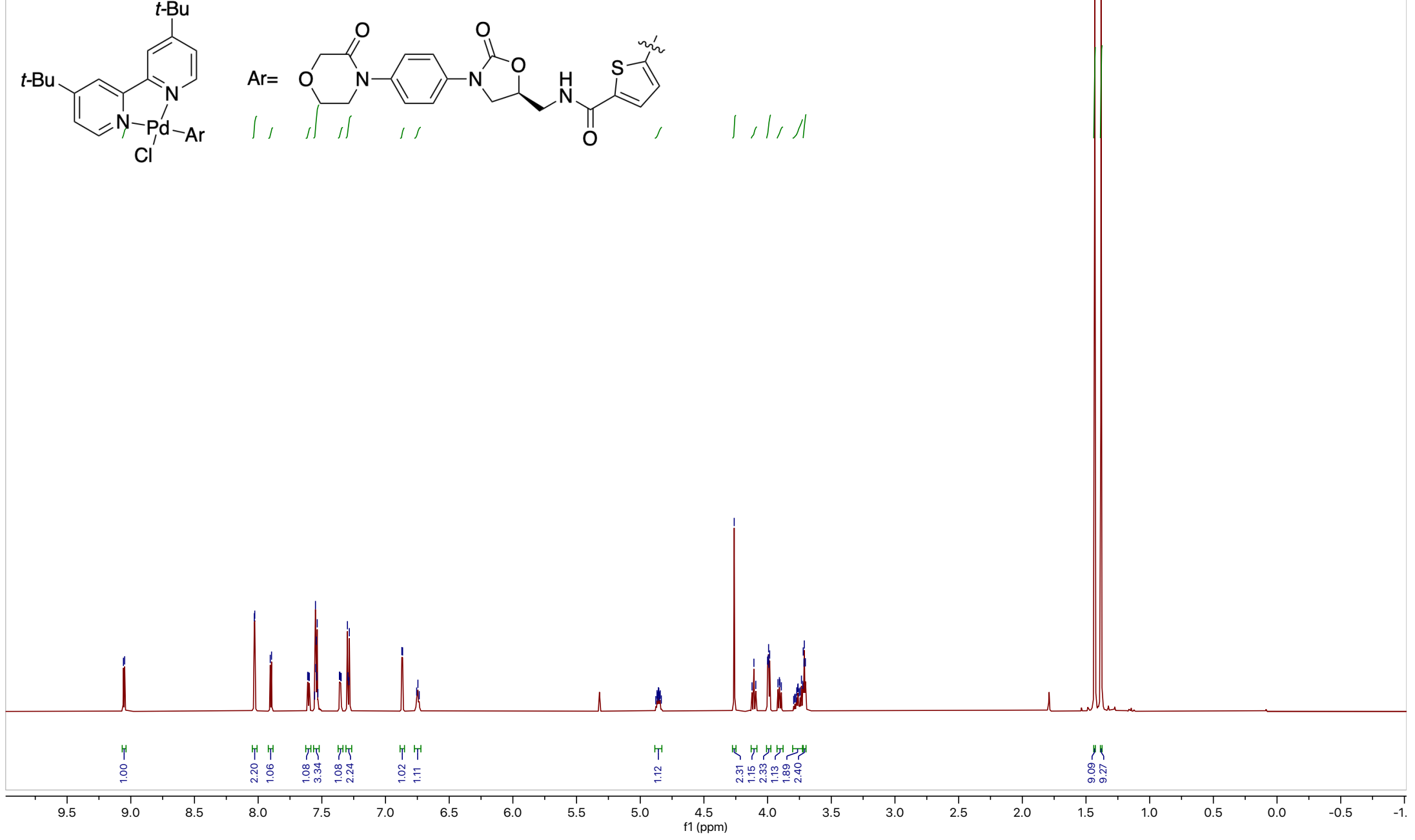


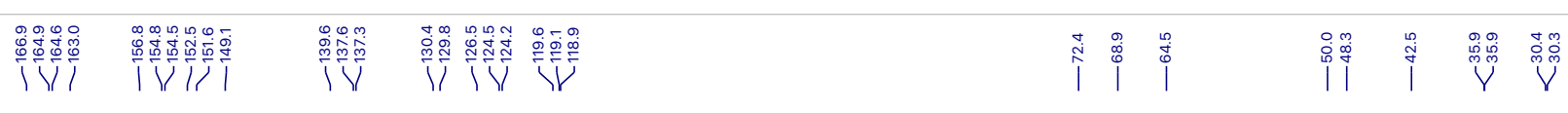

(dtbbpy)Pd(rivaroxaban)Cl (2h)

${ }^{13} \mathrm{C}$ NMR (151 MHz, $\mathrm{CD}_{2} \mathrm{Cl}_{2}$ )

(1)

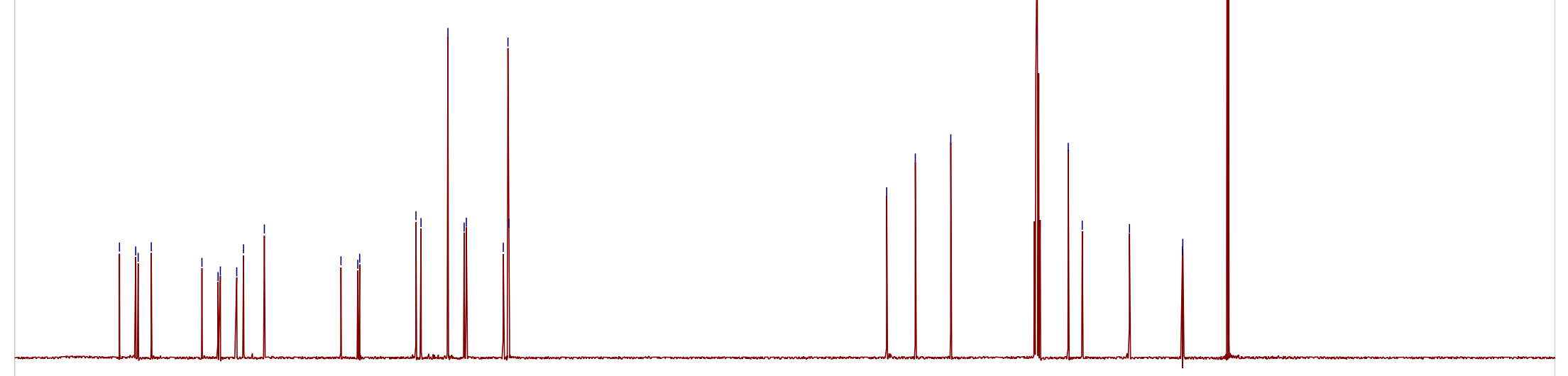

170

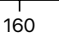

150
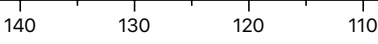

100

901 (ppm) 80

60

50

40

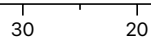




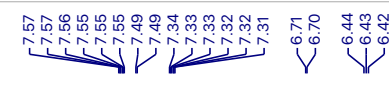

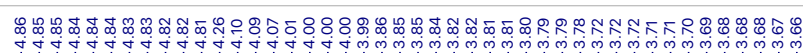

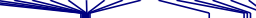

(PMe $\left._{3}\right)_{2} \mathbf{P d}$ (rivaroxaban)Cl (2i)

${ }^{1} \mathrm{H}$ NMR $\left(600 \mathrm{MHz}, \mathrm{CD}_{2} \mathrm{Cl}_{2}\right)$
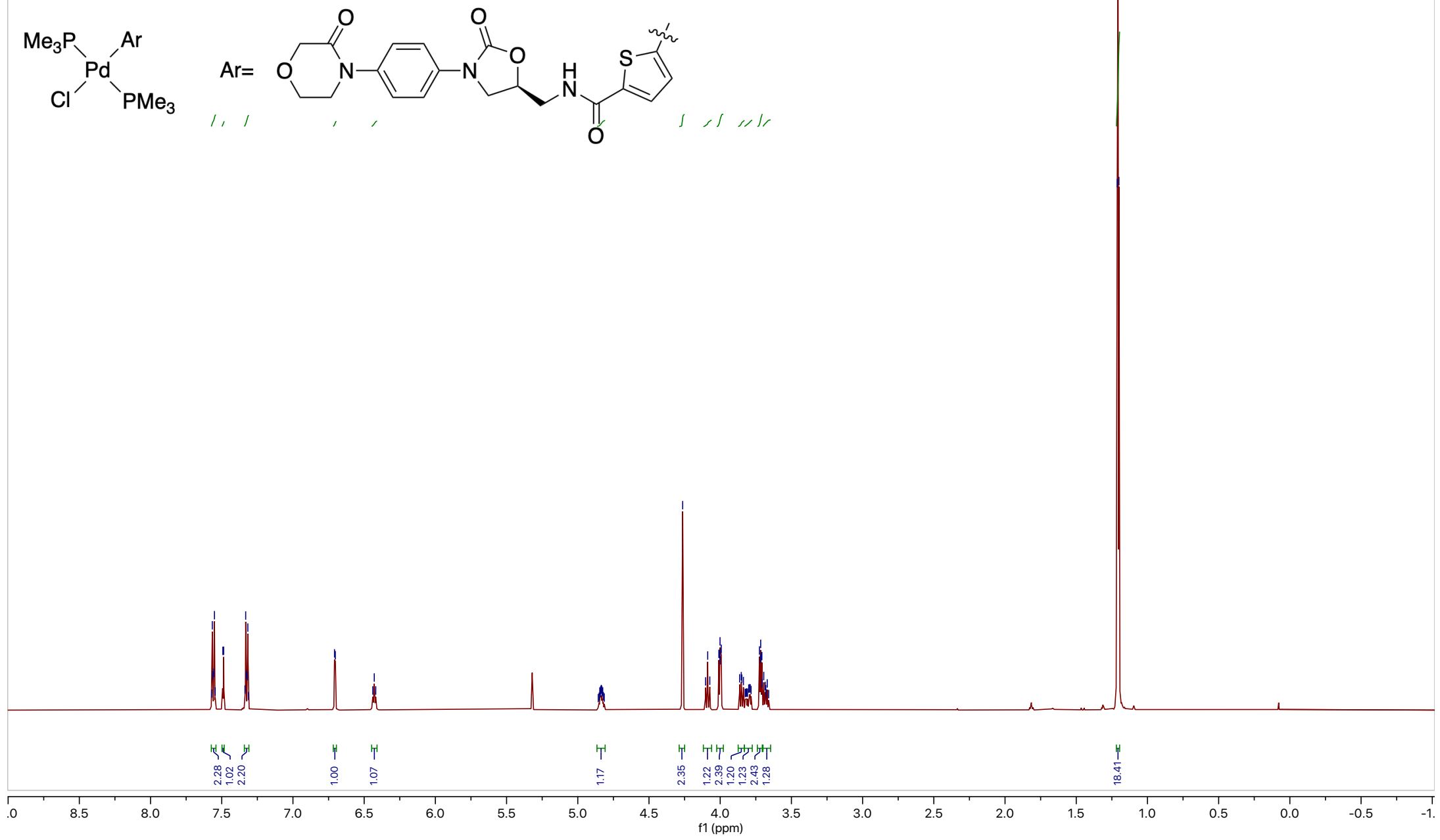


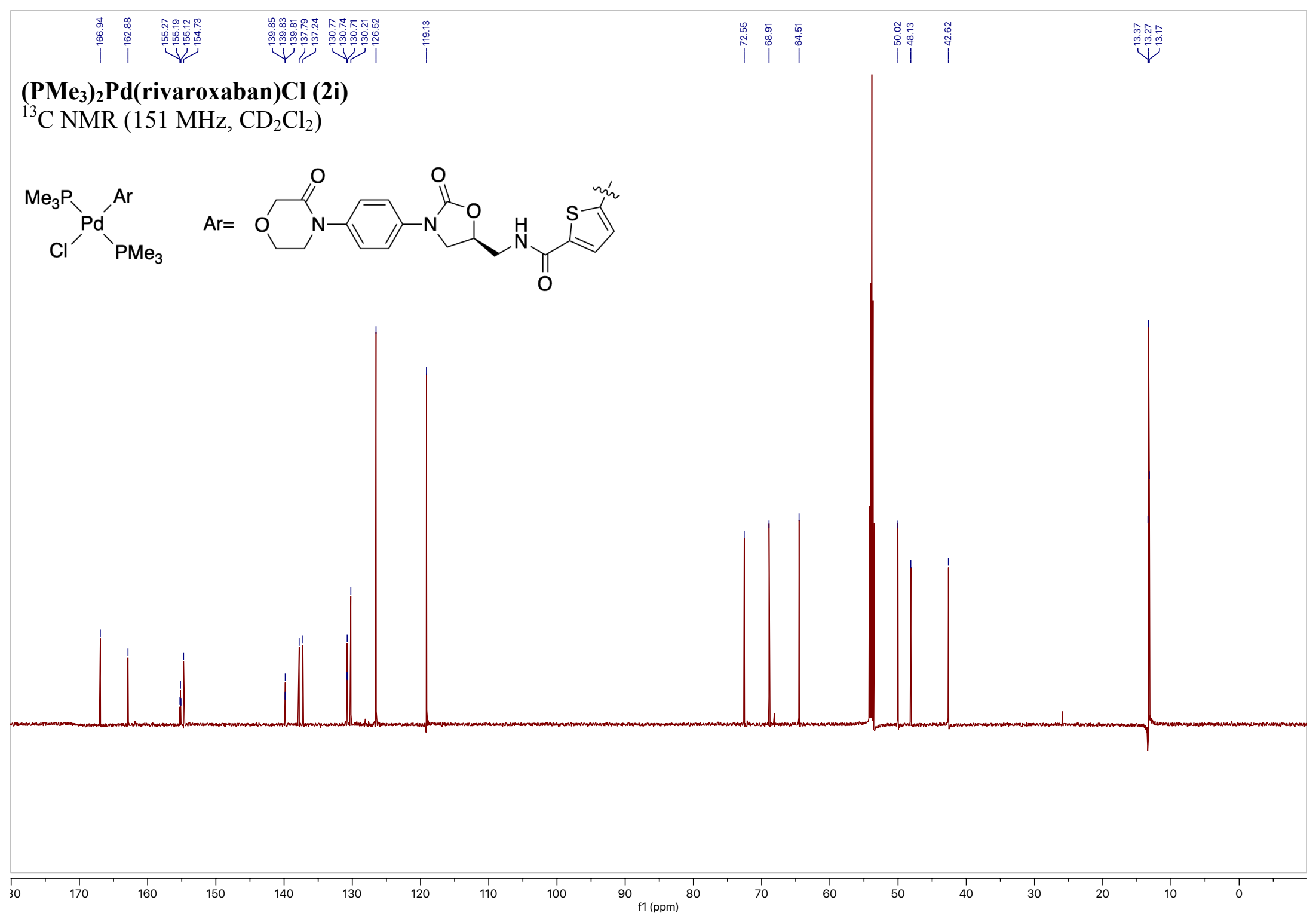


$\left(\mathrm{PMe}_{3}\right)_{2} \mathrm{Pd}($ rivaroxaban $) \mathrm{Cl}(\mathbf{2 i})$

${ }^{31} \mathrm{P}$ NMR (202 MHz, $\mathrm{CD}_{2} \mathrm{Cl}_{2}$ )

$\mathrm{Cl}^{\mathrm{Me}_{3} \mathrm{P}} \stackrel{\mathrm{Pd}}{ }{ }^{\mathrm{Ar}} \mathrm{PMe}_{3}$<smiles>Cc1ccc(C(=O)NC[C@H]2CN(c3ccc(N4CCOCC4=[V])cc3)C(=O)O2)s1</smiles>

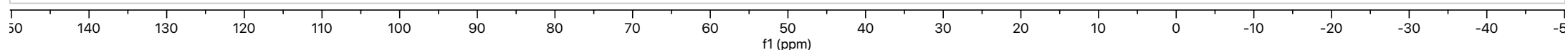




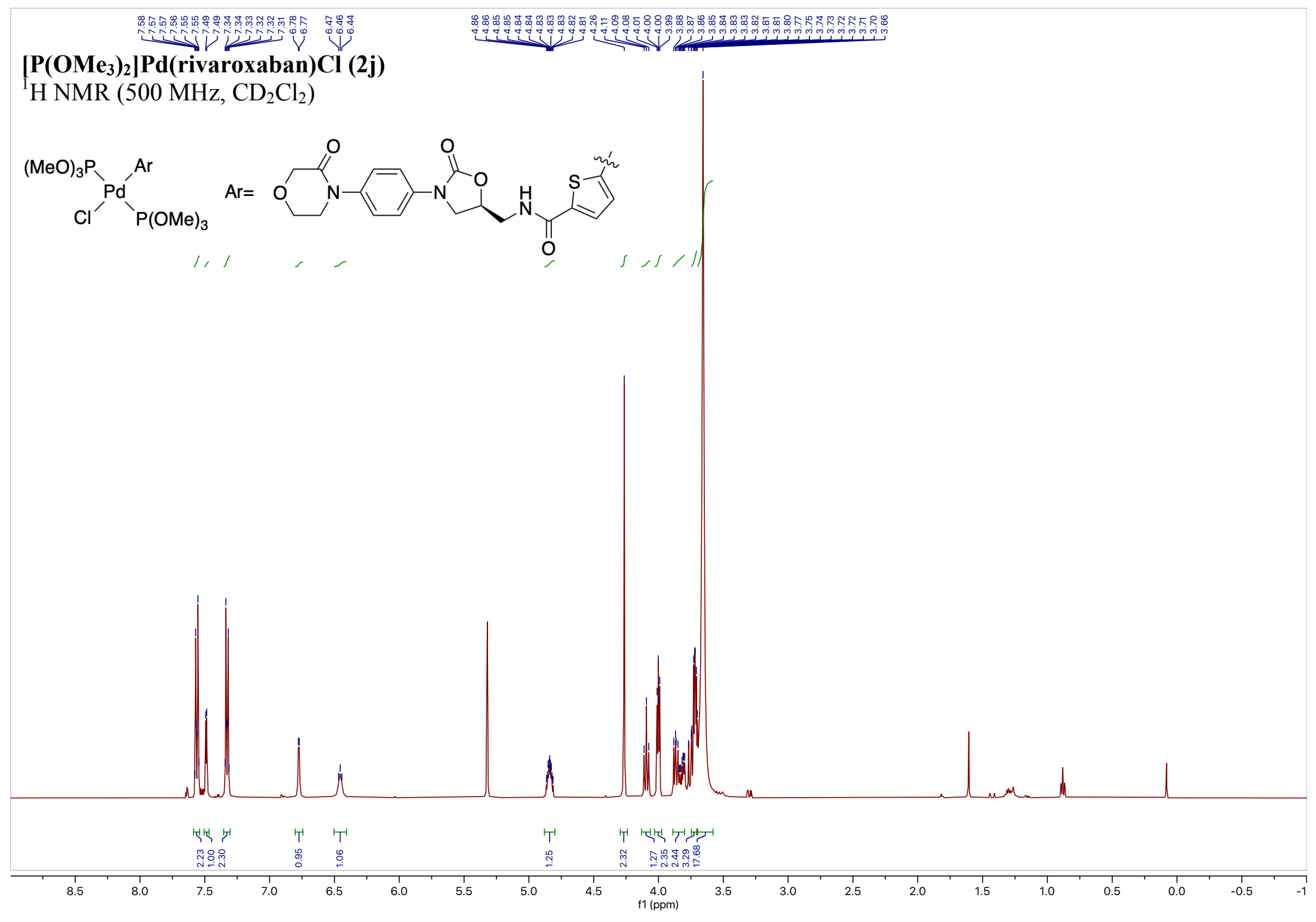




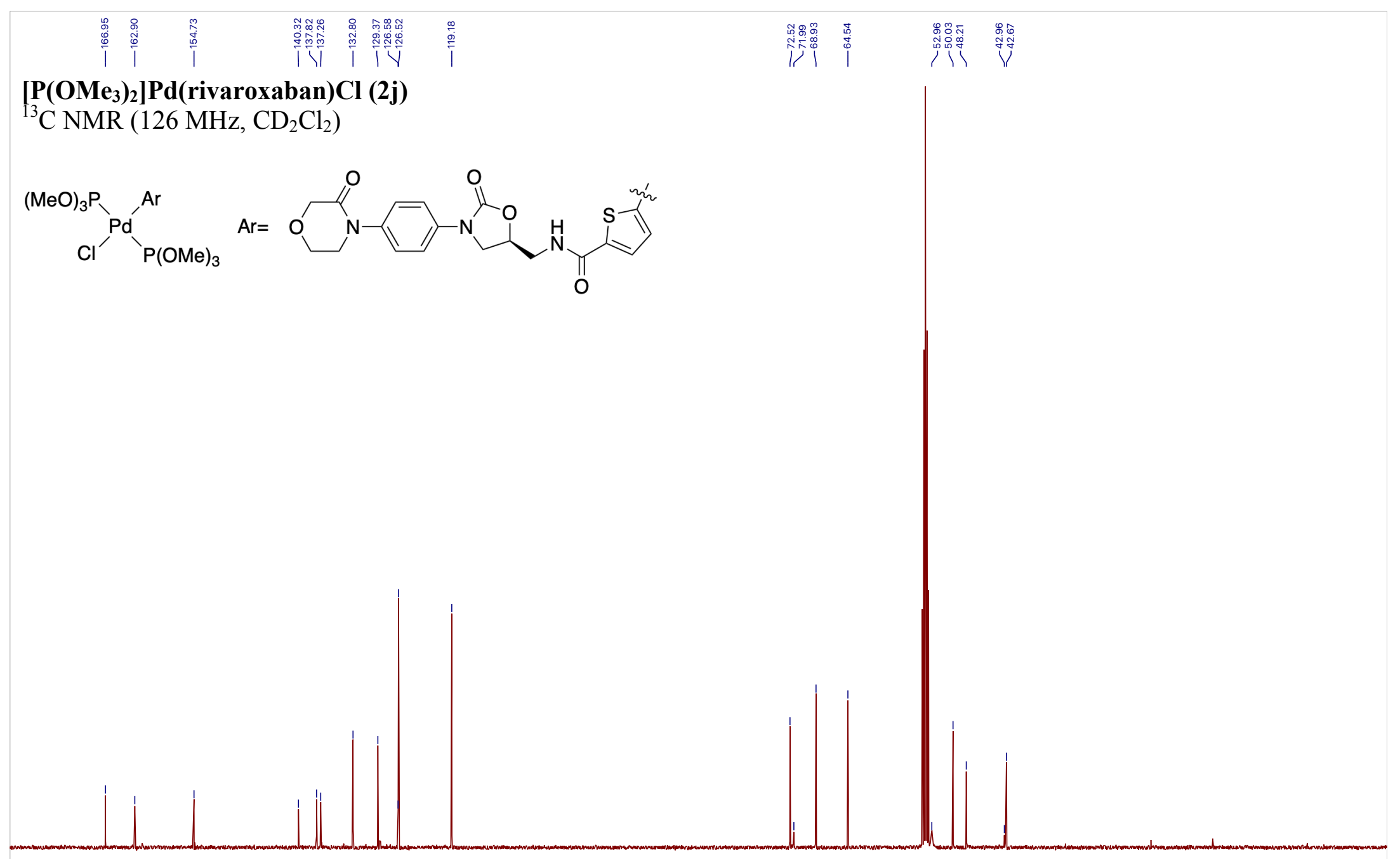

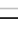
170 160 150 140

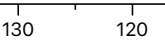

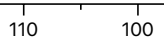
$90_{\mathrm{f} 1(\mathrm{ppm})} 80$ 70

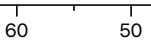
40 30 


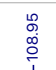

$\left[\mathbf{P}\left(\mathrm{OMe}_{3}\right)_{2}\right] \mathbf{P d}($ rivaroxaban $) \mathrm{Cl}$ (2j)

${ }^{31} \mathrm{P}$ NMR (203 MHz, $\mathrm{CD}_{2} \mathrm{Cl}_{2}$ )

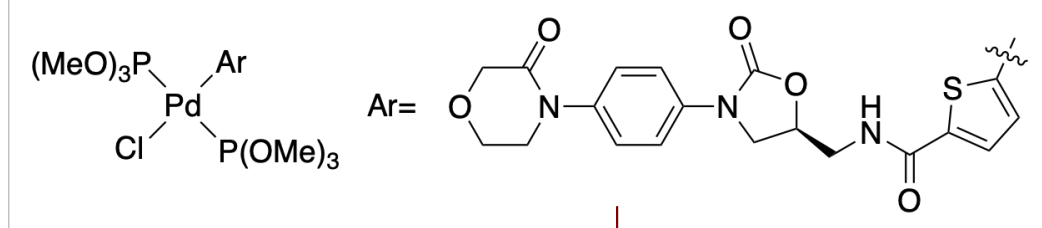

W.y.

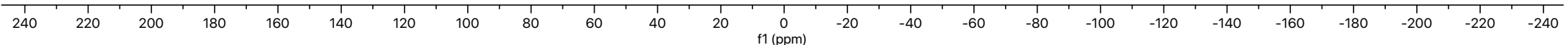




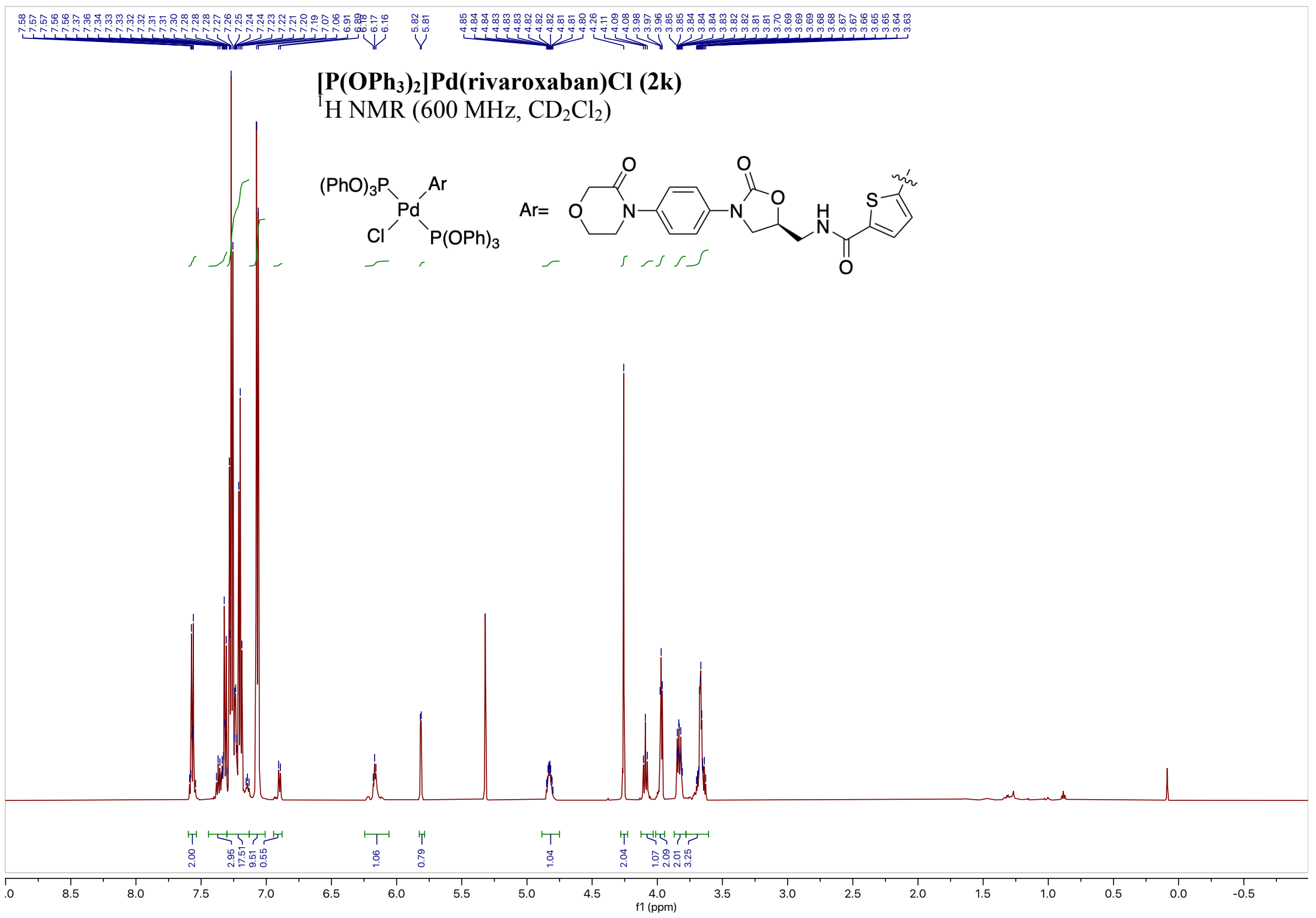




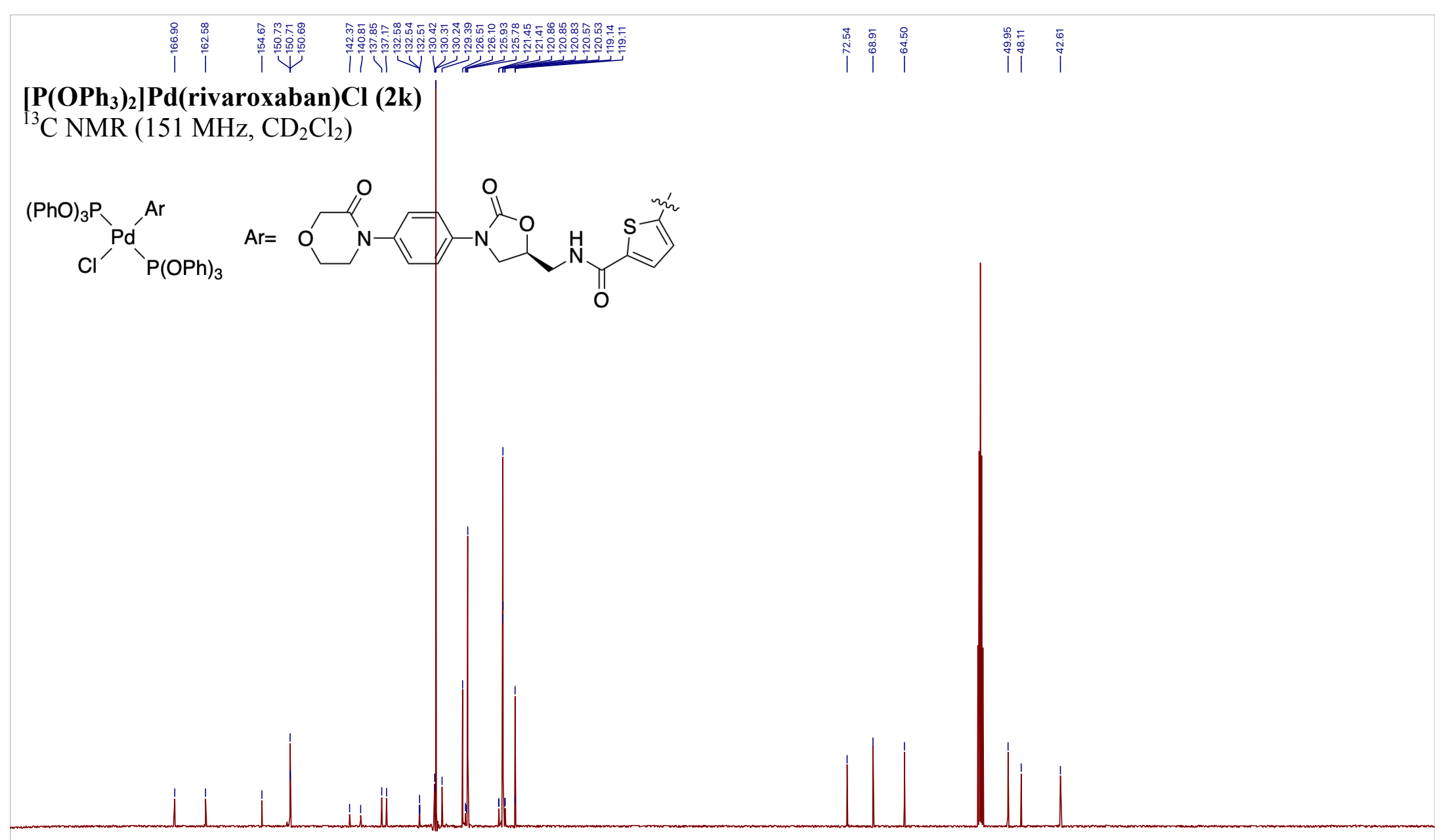




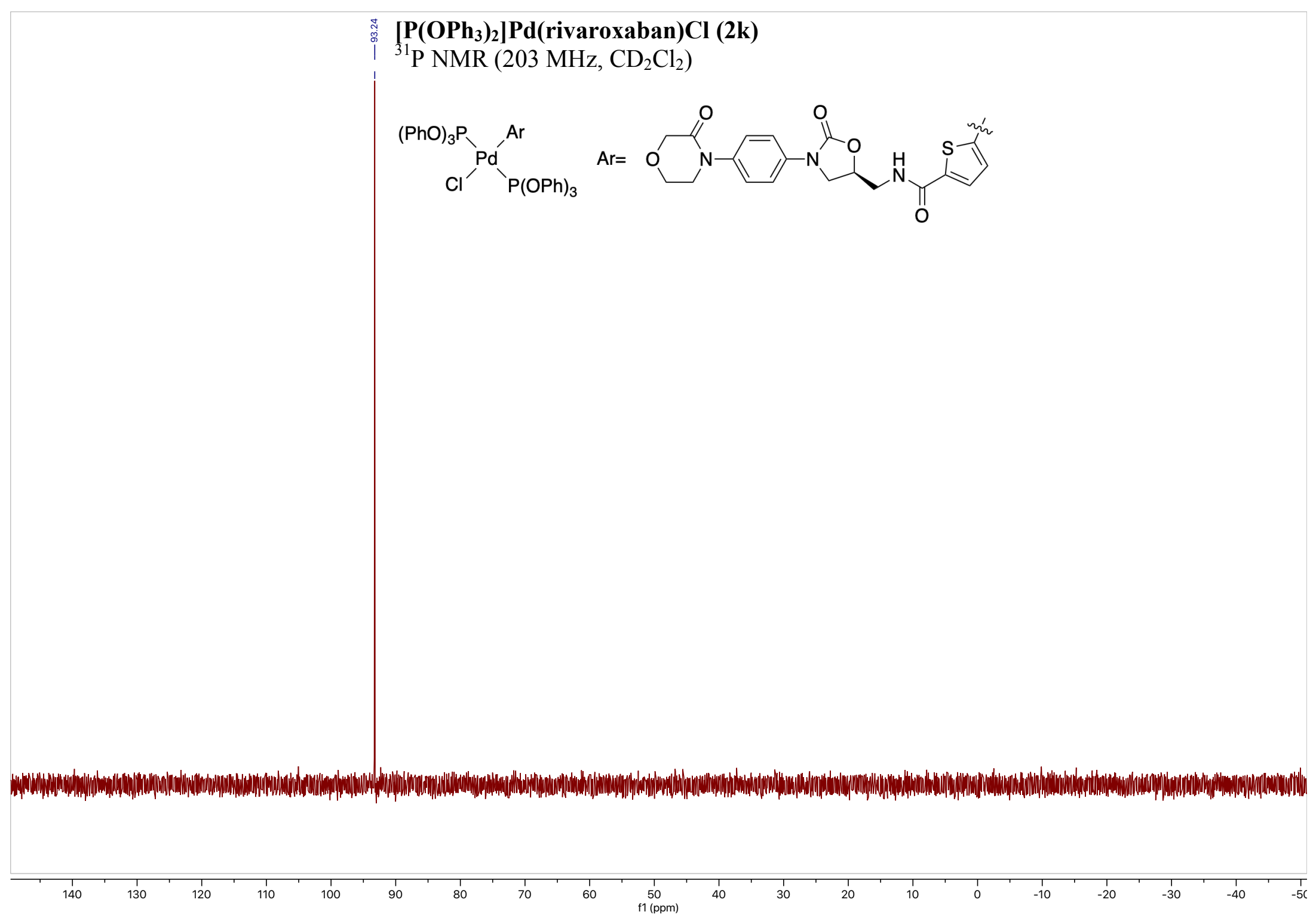


(RuPhos)Pd(X4)Br (3)

${ }^{1} \mathrm{H}$ NMR $\left(600 \mathrm{MHz}, \mathrm{CD}_{2} \mathrm{Cl}_{2}\right)$

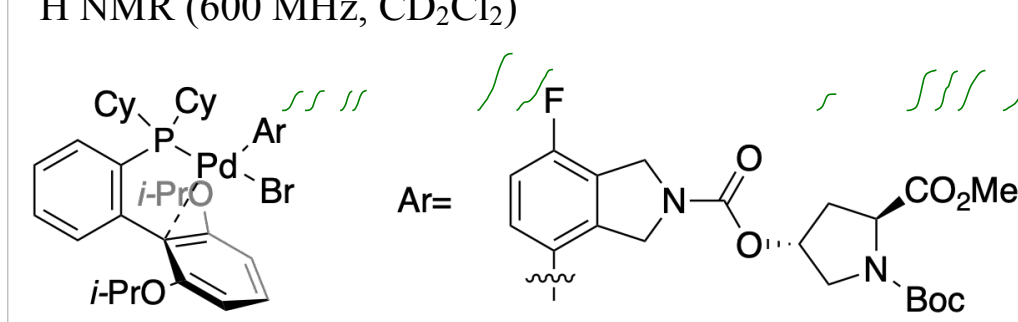

$\mathrm{Ar}=$

$$
\mathrm{O}_{\mathrm{N}}
$$
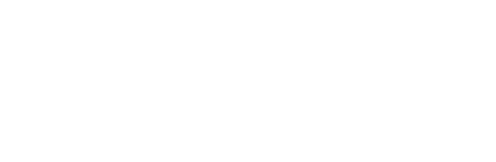


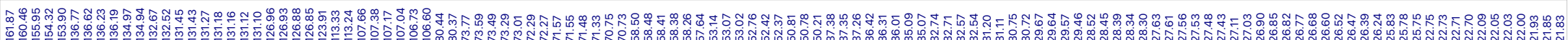

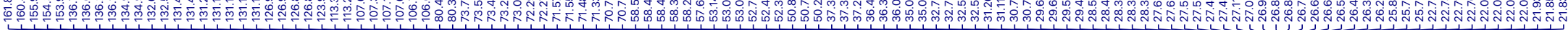

\section{(RuPhos)Pd(X4)Br (3)}

${ }^{13} \mathrm{C}$ NMR $\left(151 \mathrm{MHz}, \mathrm{CD}_{2} \mathrm{Cl}_{2}\right)$

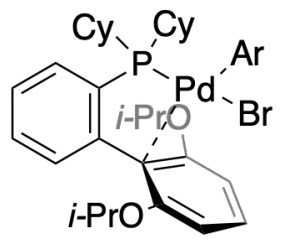

$\operatorname{Ar}=$<smiles>COC(=O)[C@@H]1C[C@@H](OC(=O)N2Cc3c(C)ccc(F)c3C2)CN1C(=O)OC(C)(C)C</smiles>

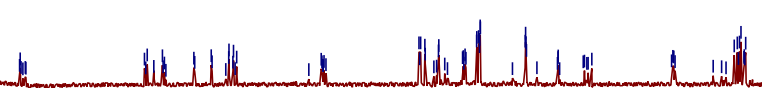


(RuPhos)Pd(X4)Br (3)

${ }^{19} \mathrm{~F}$ NMR (565 MHz, $\mathrm{CD}_{2} \mathrm{Cl}_{2}$ )

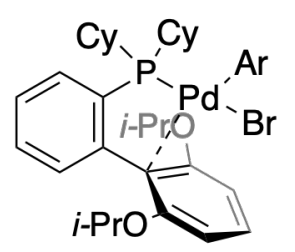

$\operatorname{Ar}=$

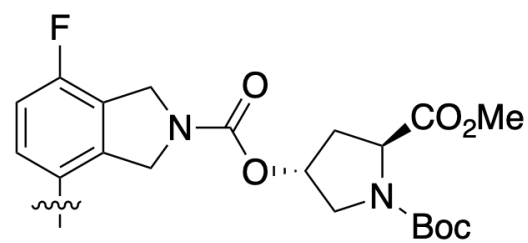

Boc

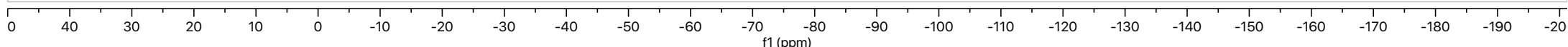


(RuPhos)Pd(X4)Br (3)

${ }^{31} \mathrm{P}$ NMR (203 MHz, $\mathrm{CD}_{2} \mathrm{Cl}_{2}$ )

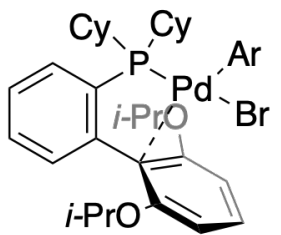

$\operatorname{Ar}=$<smiles>COC(=O)C1CC(OC(=O)N2Cc3c(C)ccc(F)c3C2)CN1C(=O)OCc1ccccc1</smiles> 


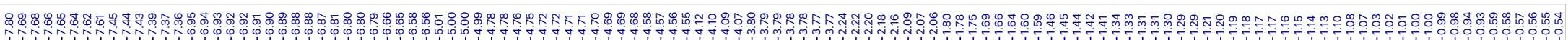
(RuPhos)Pd(X14)I (4)

${ }^{1} \mathrm{H}$ NMR $\left(600 \mathrm{MHz}, \mathrm{CD}_{2} \mathrm{Cl}_{2}\right.$ )

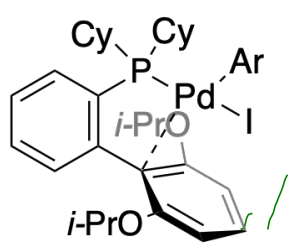<smiles></smiles>
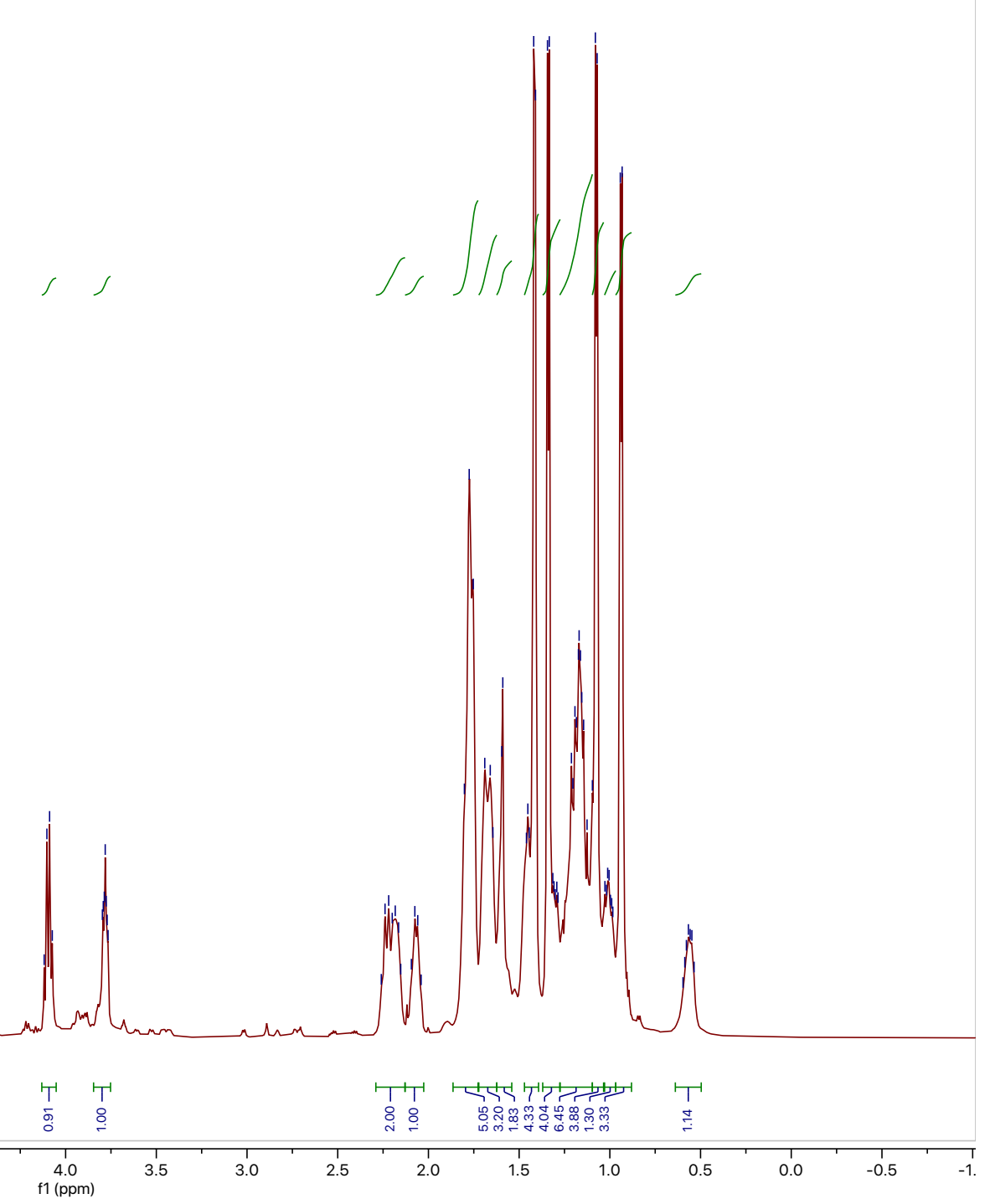


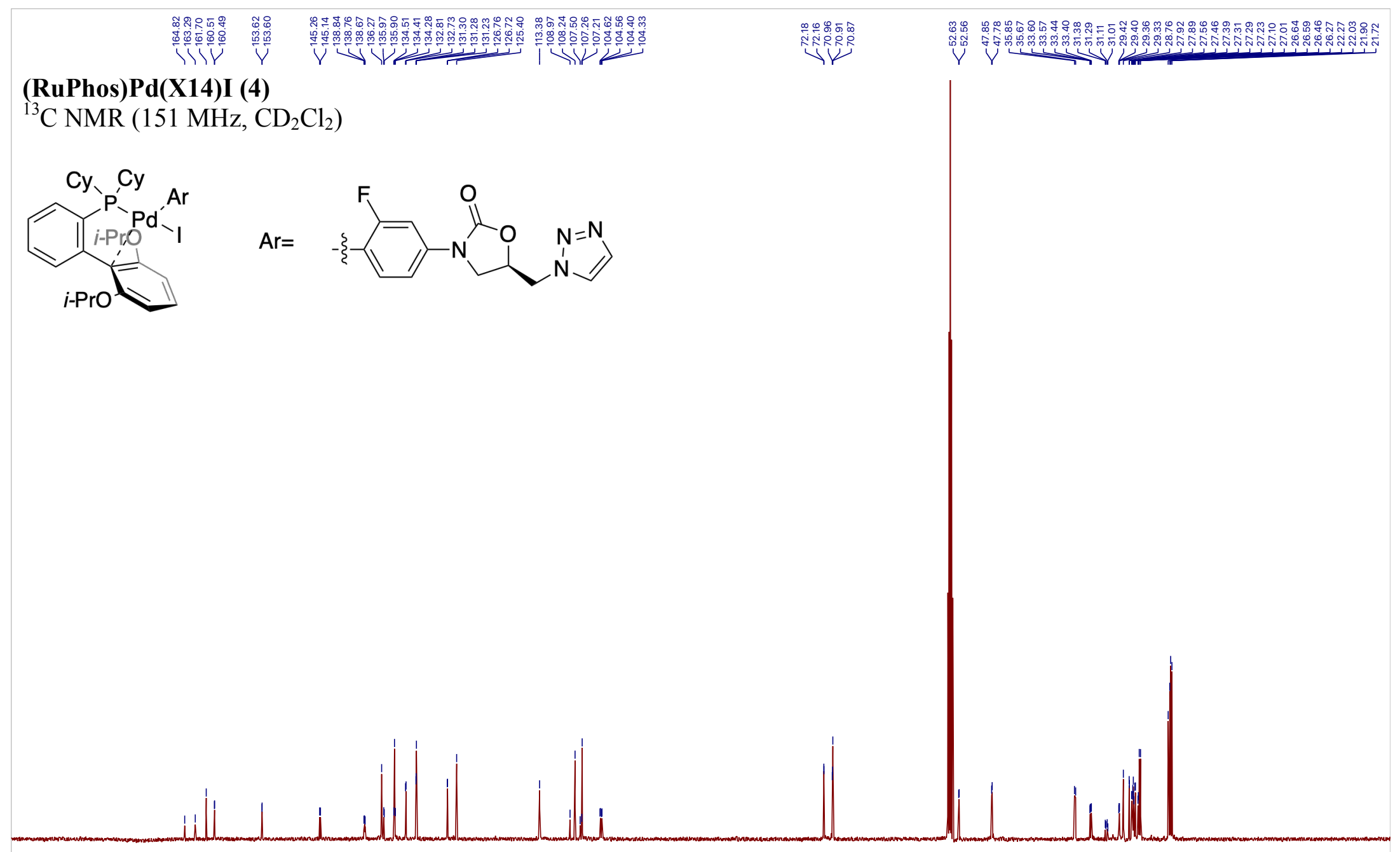


(RuPhos)Pd(X14)I (4)

${ }^{9} \mathrm{~F}$ NMR (565 MHz, $\mathrm{CD}_{2} \mathrm{Cl}_{2}$ )

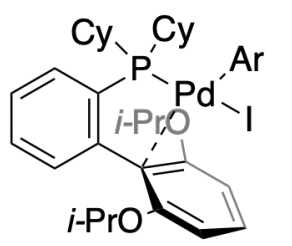

$\mathrm{Ar}=$
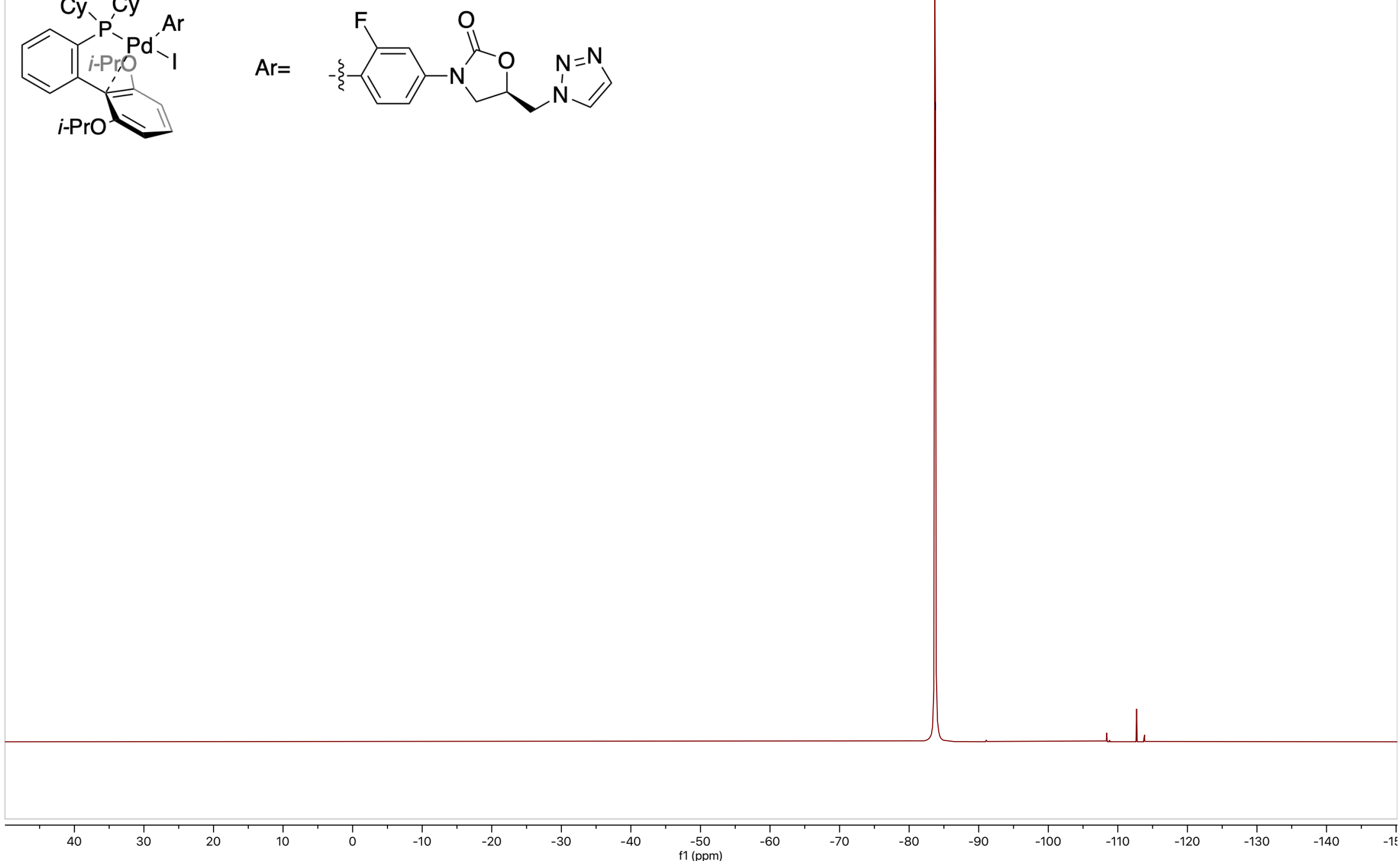
(RuPhos)Pd(X14)I (4)

${ }^{31} \mathrm{P}$ NMR $\left(203 \mathrm{MHz}, \mathrm{CD}_{2} \mathrm{Cl}_{2}\right)$

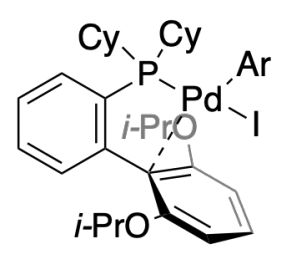

$\mathrm{Ar}=$

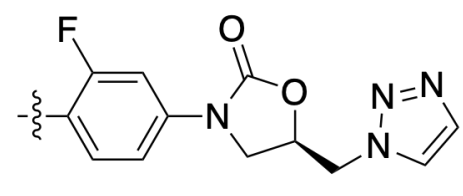

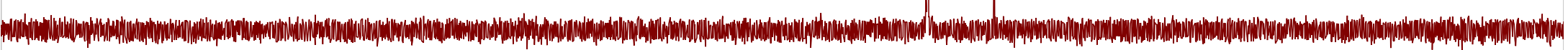

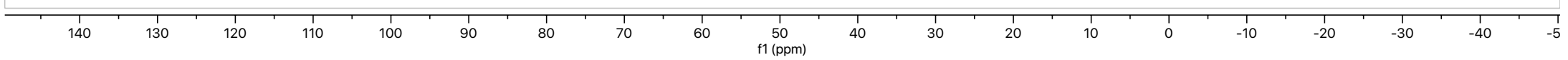


(RuPhos)Pd(indometacin)Cl (5)

${ }^{1} \mathrm{H}$ NMR $\left(500 \mathrm{MHz}, \mathrm{CD}_{2} \mathrm{Cl}_{2}\right.$ )<smiles>COc1ccc2c(c1)c(CC(=O)O)c(C)n2C(=O)c1ccc(C(C)(F)Cl)cc1</smiles>
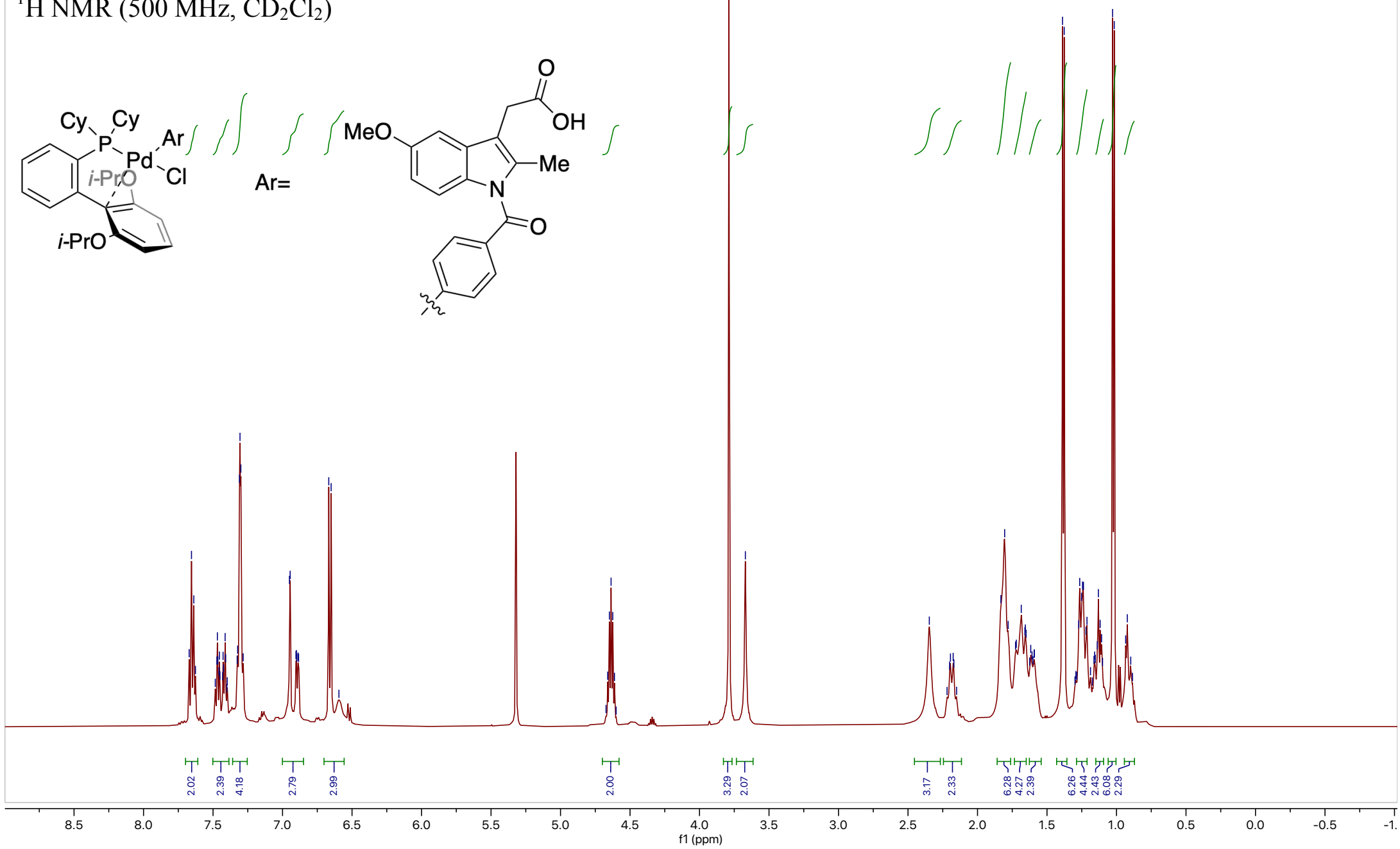


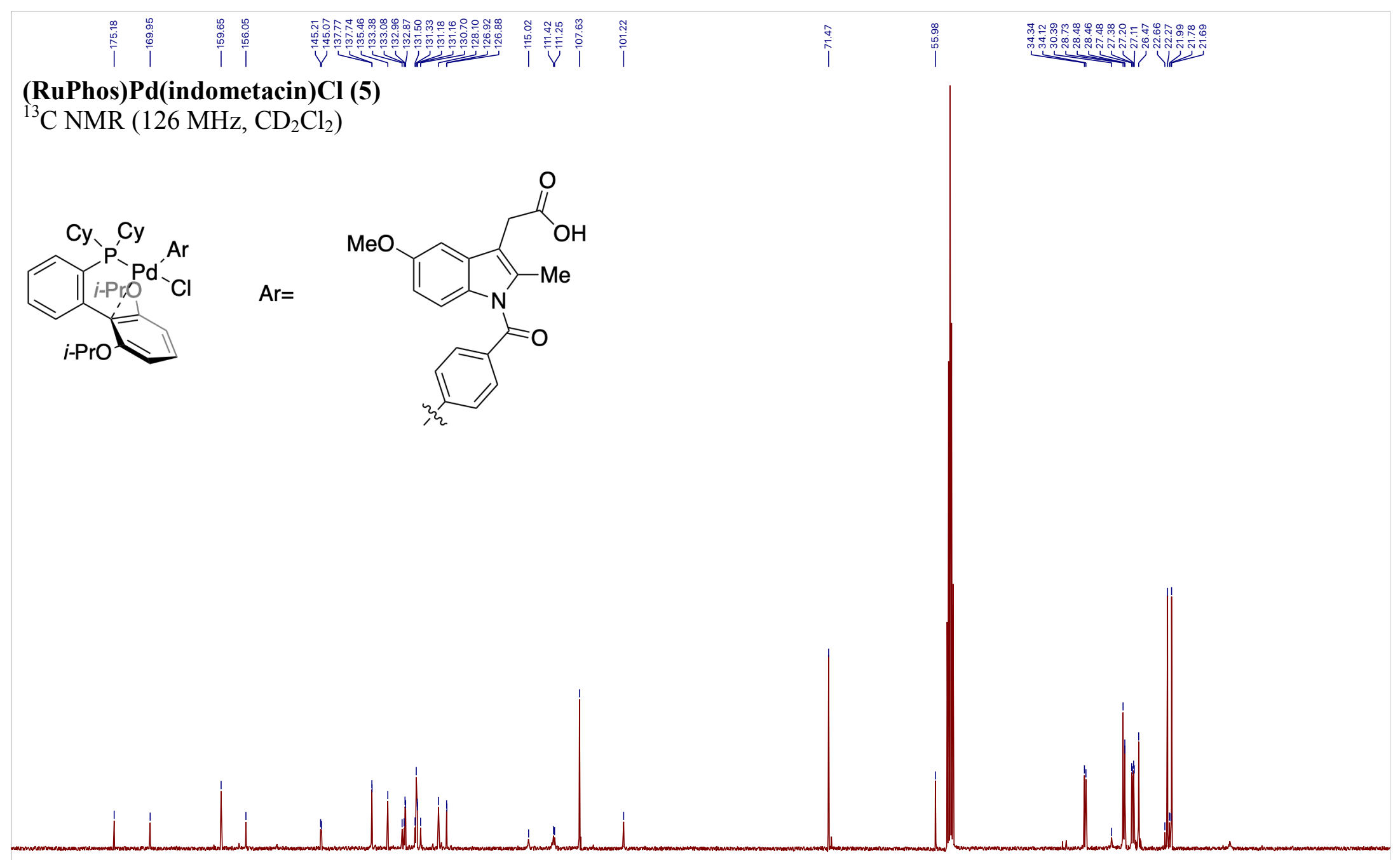

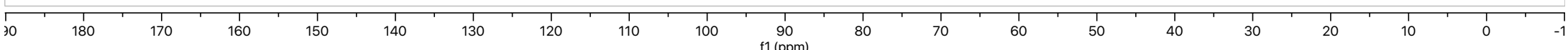


(RuPhos)Pd(indometacin)Cl (5)

${ }^{31} \mathrm{P}$ NMR (203 MHz, $\mathrm{CD}_{2} \mathrm{Cl}_{2}$ )

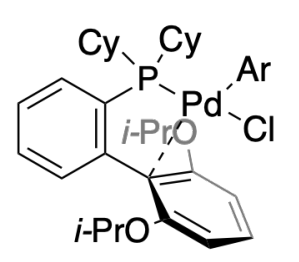

$\mathrm{Ar}=$

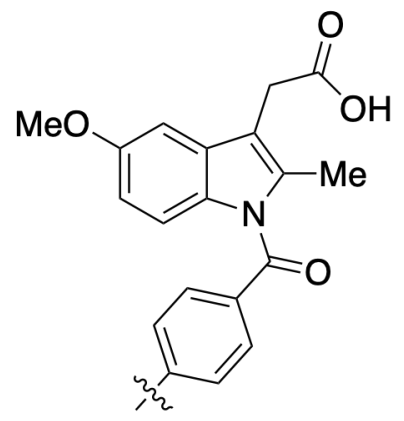


(XPhos)Pd[(4-CF 3 ) Ph] Cl (6)

${ }^{1} \mathrm{H}$ NMR $\left(500 \mathrm{MHz}, \mathrm{CD}_{2} \mathrm{Cl}_{2}\right.$ )

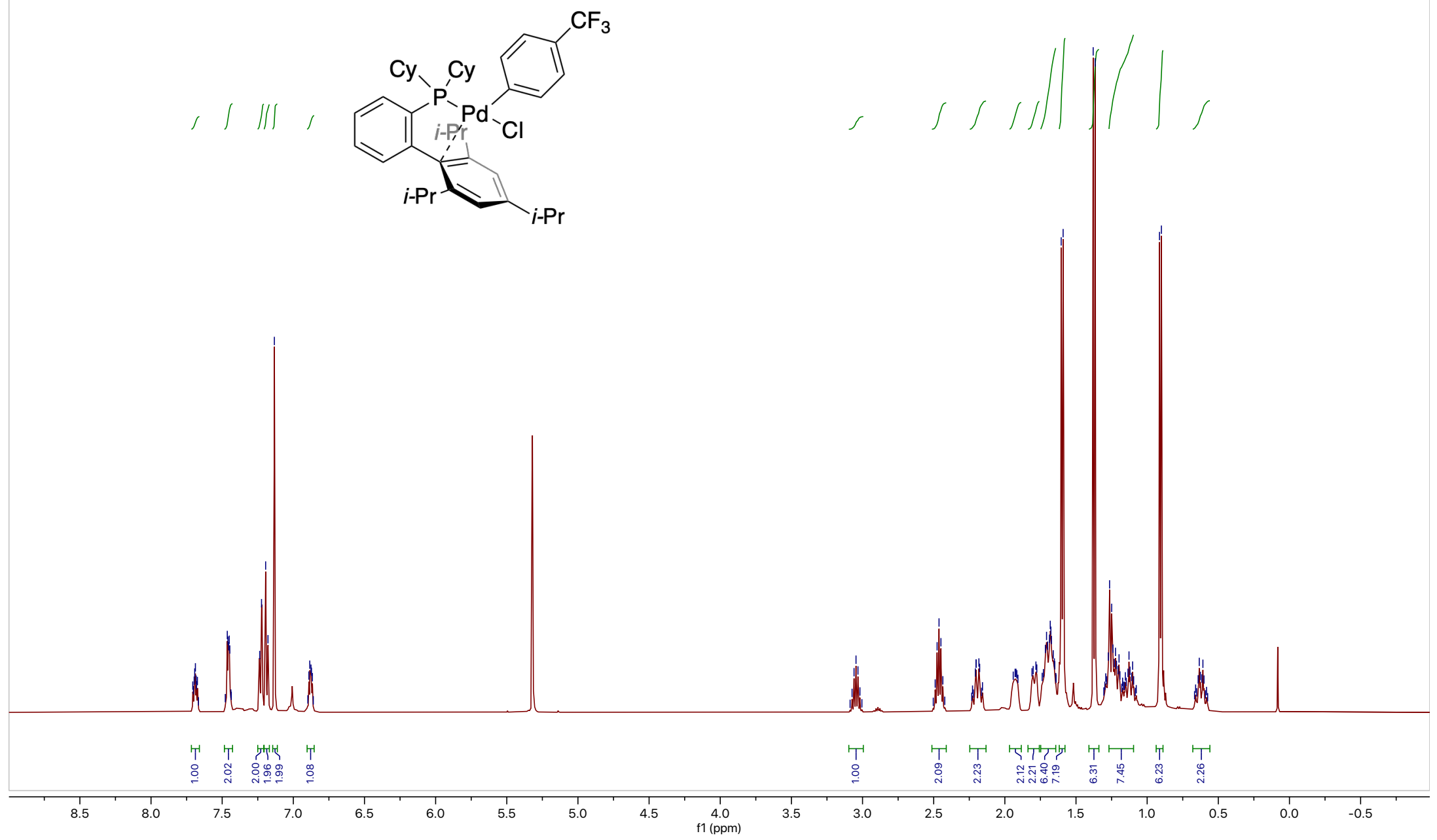




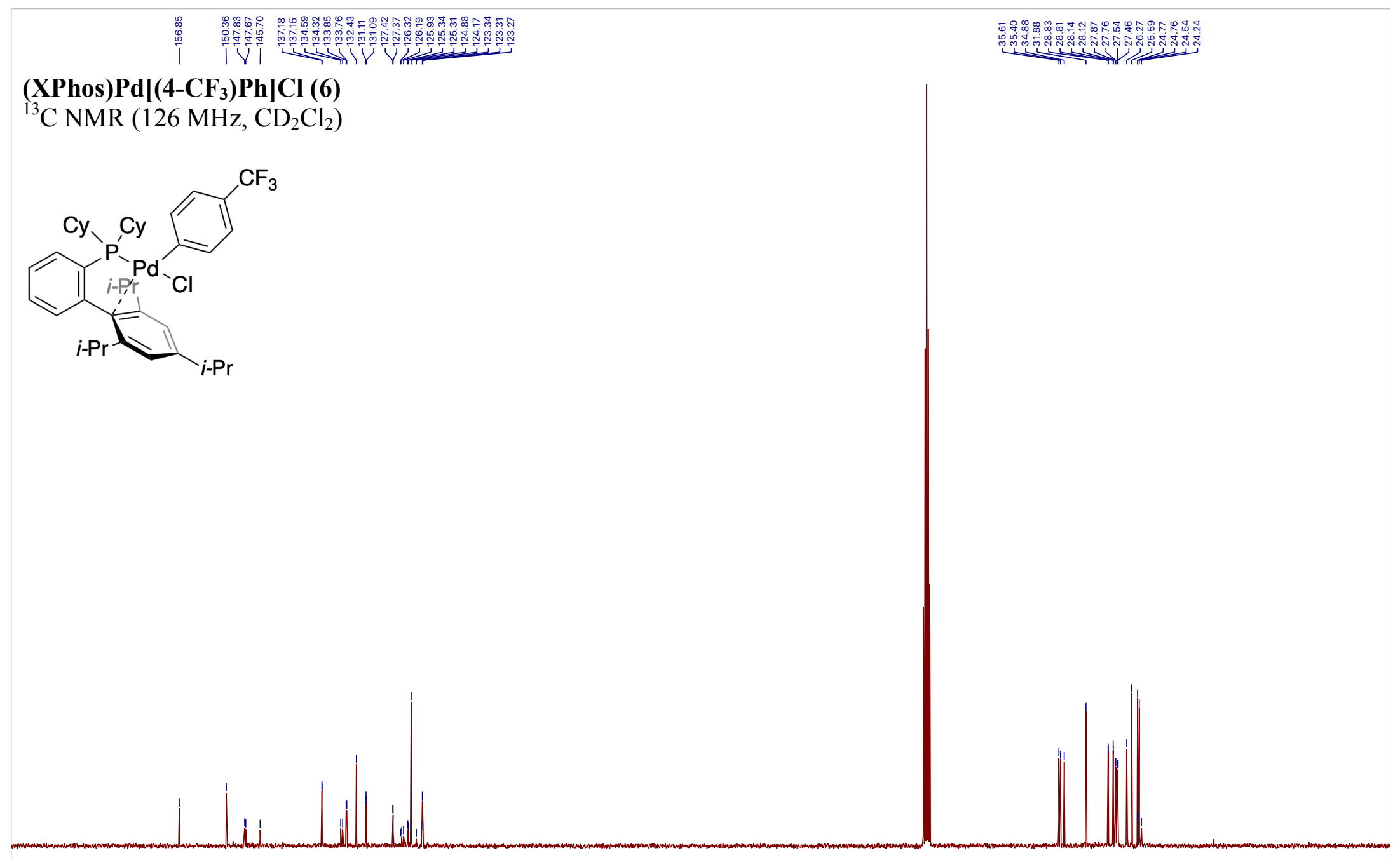


(XPhos)Pd[(4-CF 3$) P h] C l(6)$

${ }^{19} \mathrm{~F}$ NMR (471 MHz, $\mathrm{CD}_{2} \mathrm{Cl}_{2}$ )
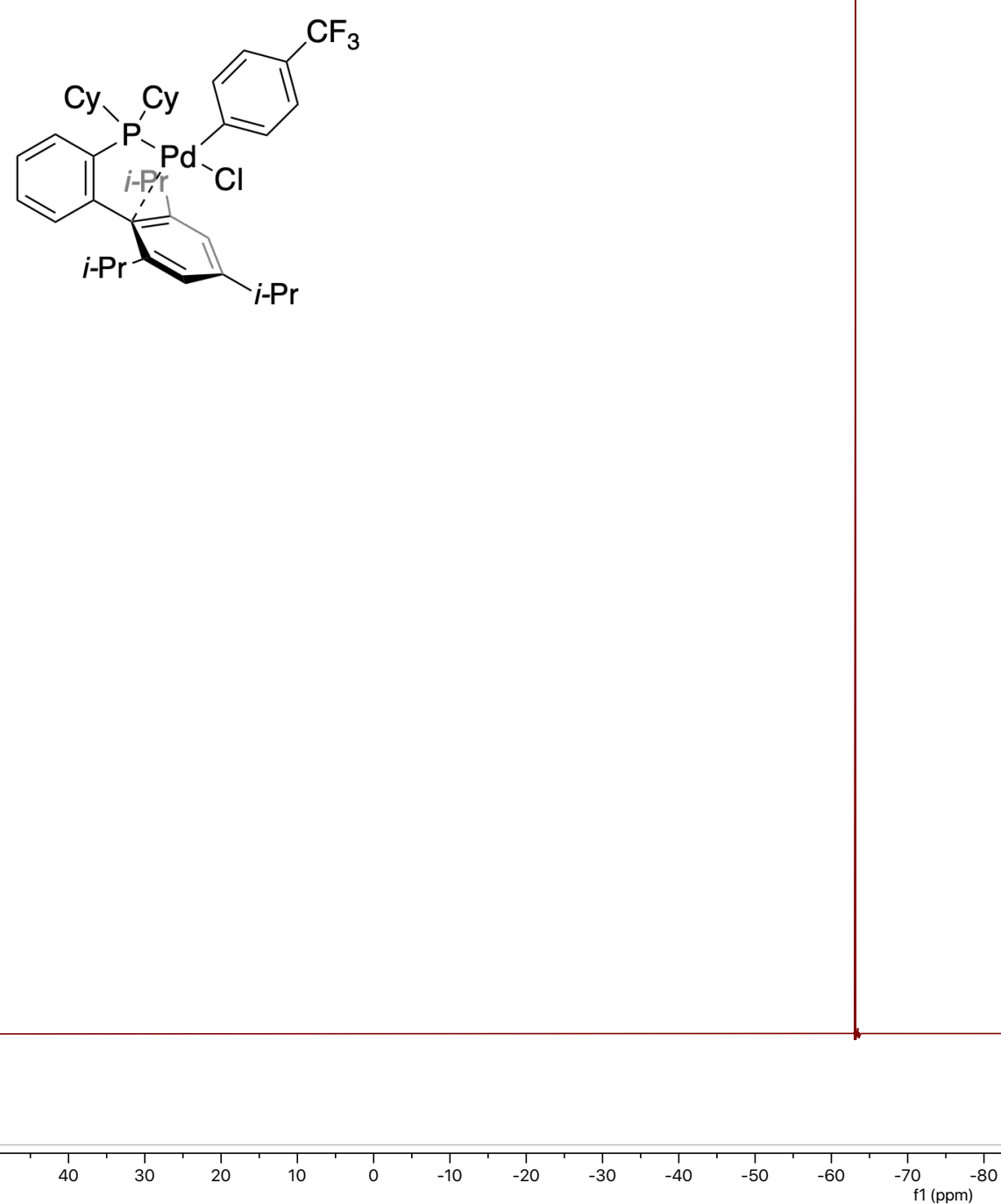
(XPhos)Pd[(4-CF $) \mathrm{Ph}] \mathrm{Cl}(6)$

${ }^{31} \mathrm{P}$ NMR $\left(203 \mathrm{MHz}, \mathrm{CD}_{2} \mathrm{Cl}_{2}\right)$
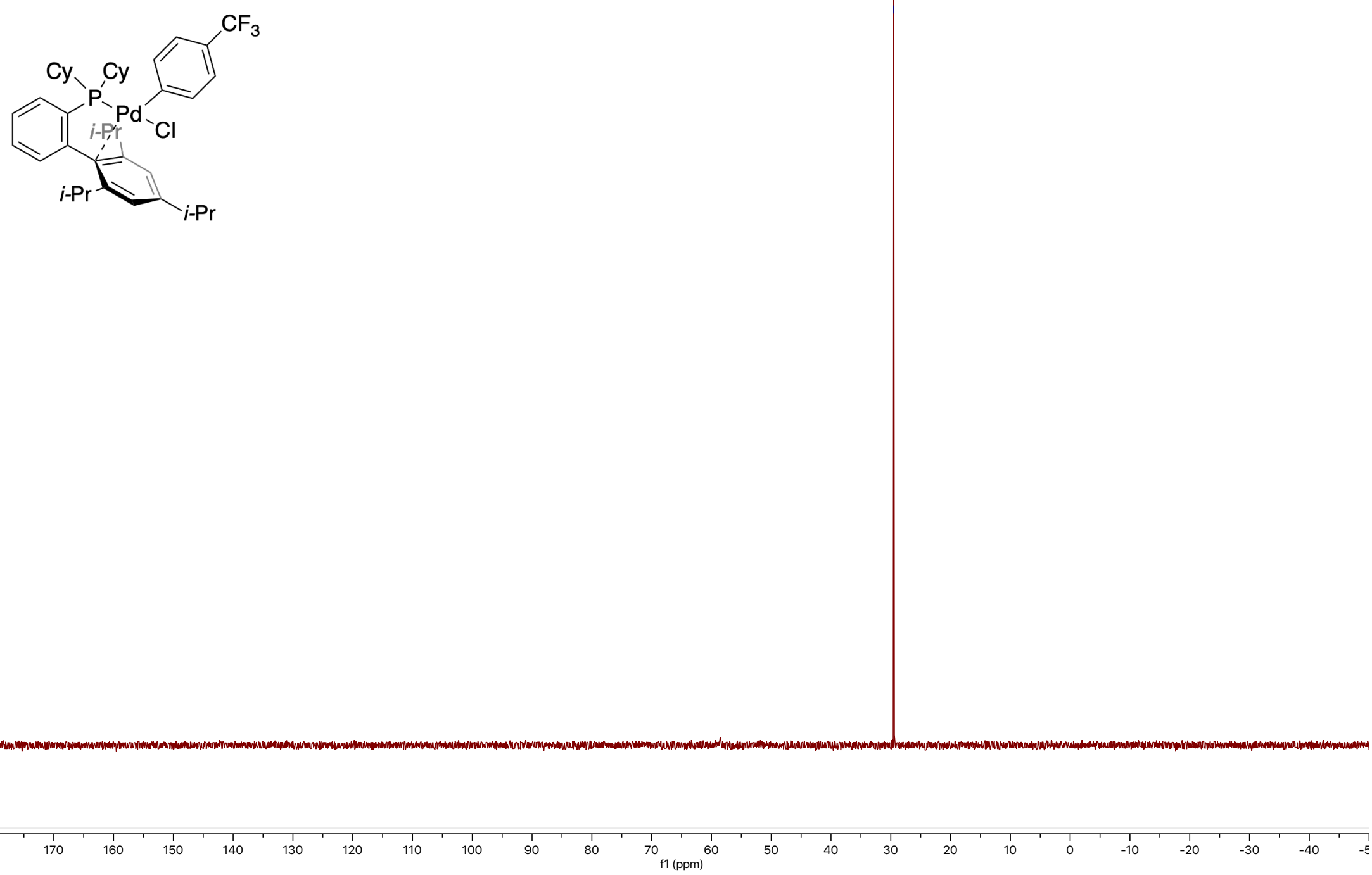


\section{(XPhos) Pd[(4-CF 3 ) Ph] Br (7)}

${ }^{1} \mathrm{H}$ NMR $\left(600 \mathrm{MHz}, \mathrm{CD}_{2} \mathrm{Cl}_{2}\right.$ )

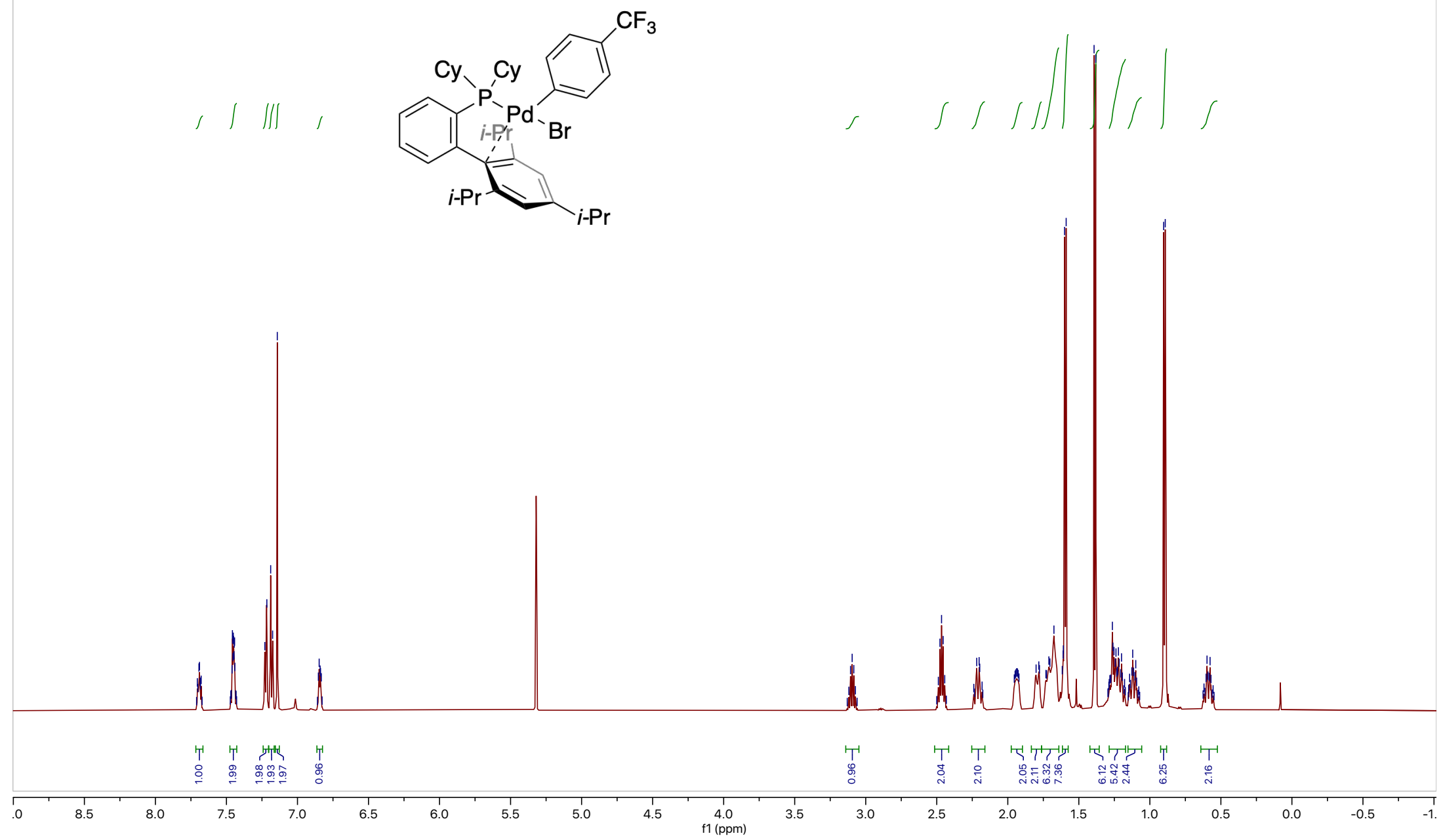




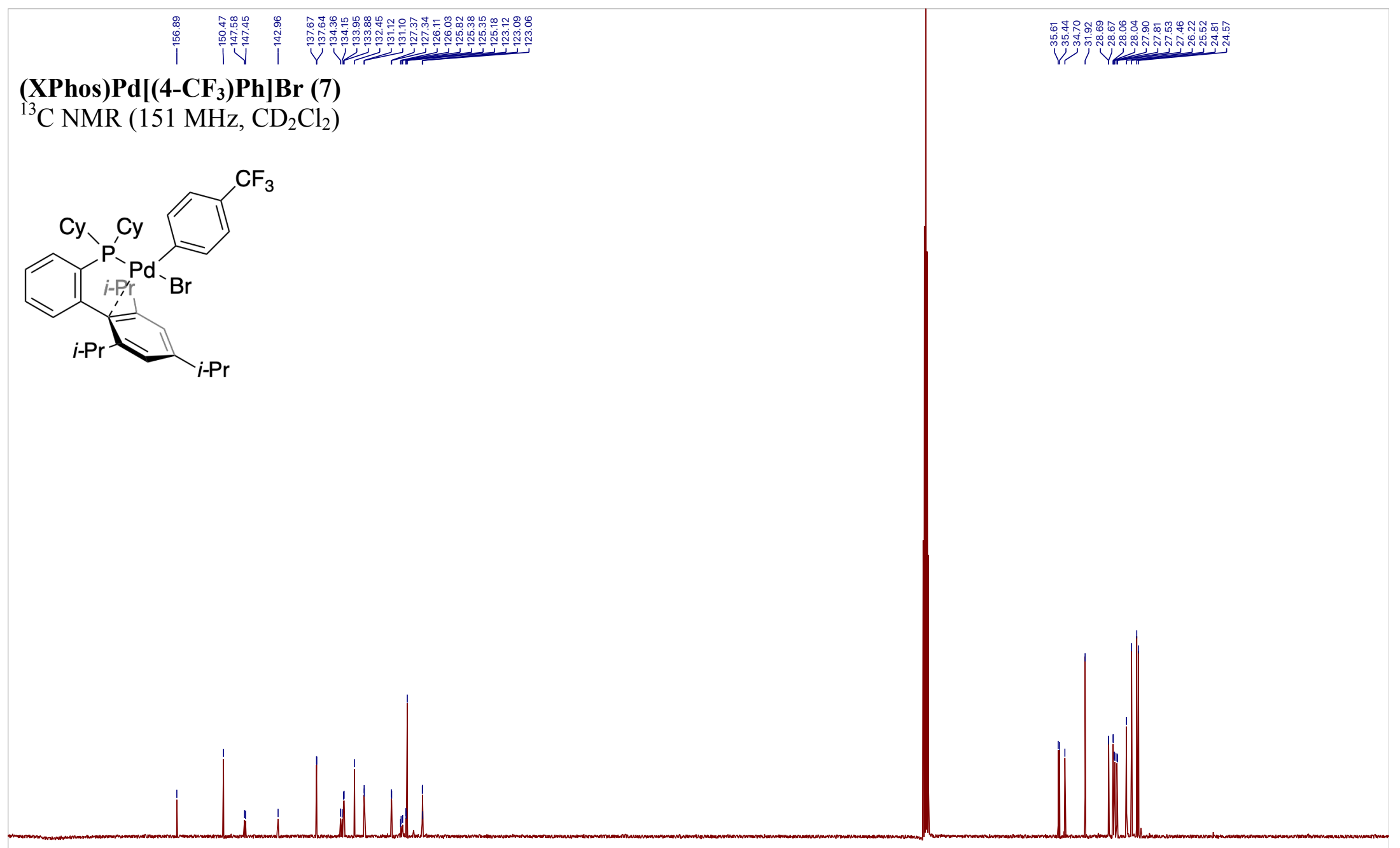


(XPhos)Pd[(4-CF 3$) P h] B r(7)$

${ }^{19} \mathrm{~F}$ NMR (565 MHz, $\mathrm{CD}_{2} \mathrm{Cl}_{2}$ )
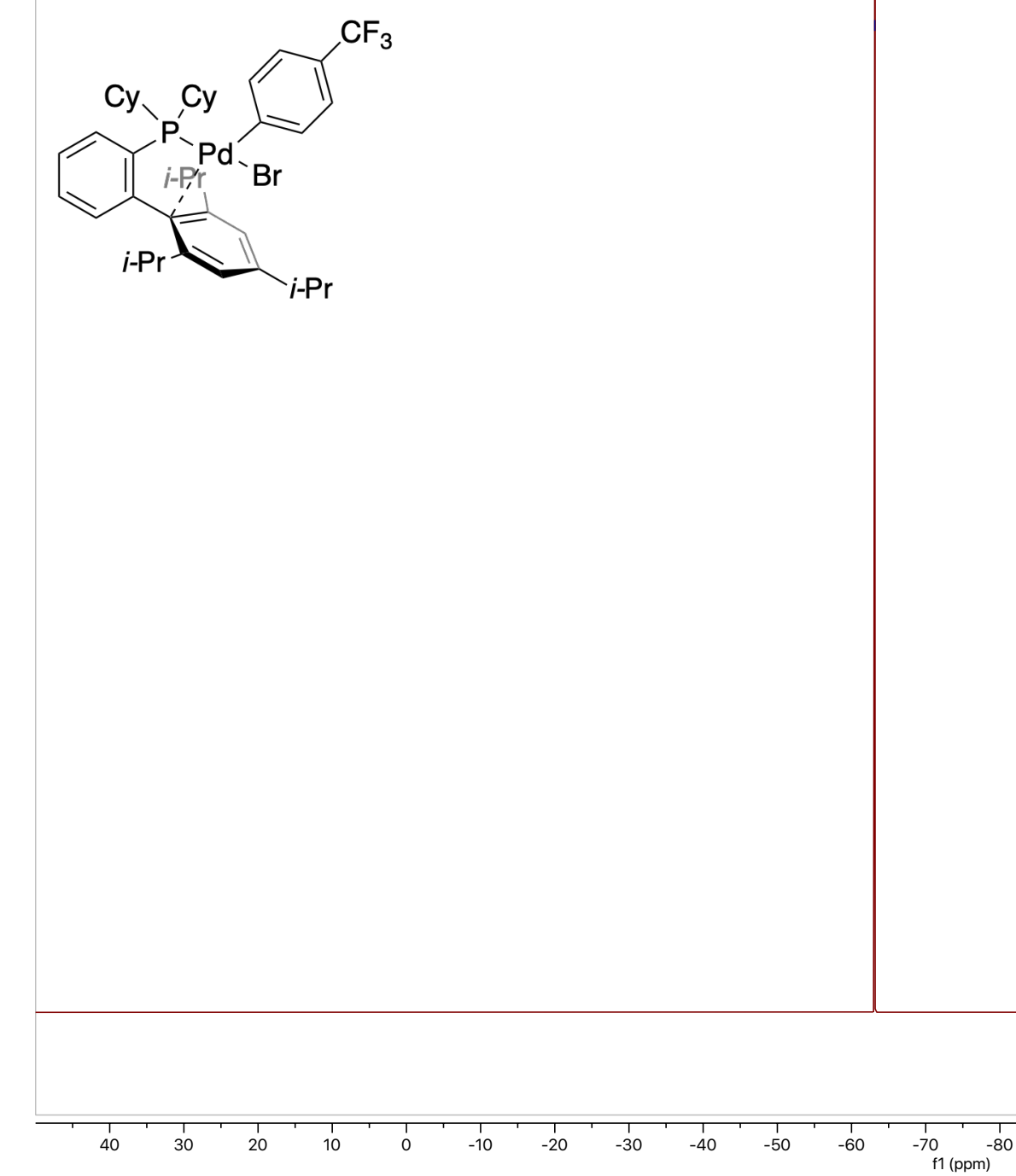
(XPhos)Pd[(4-CF 3$) P h] B r(7)$

${ }^{31} \mathrm{P}$ NMR (203 MHz, $\mathrm{CD}_{2} \mathrm{Cl}_{2}$ )
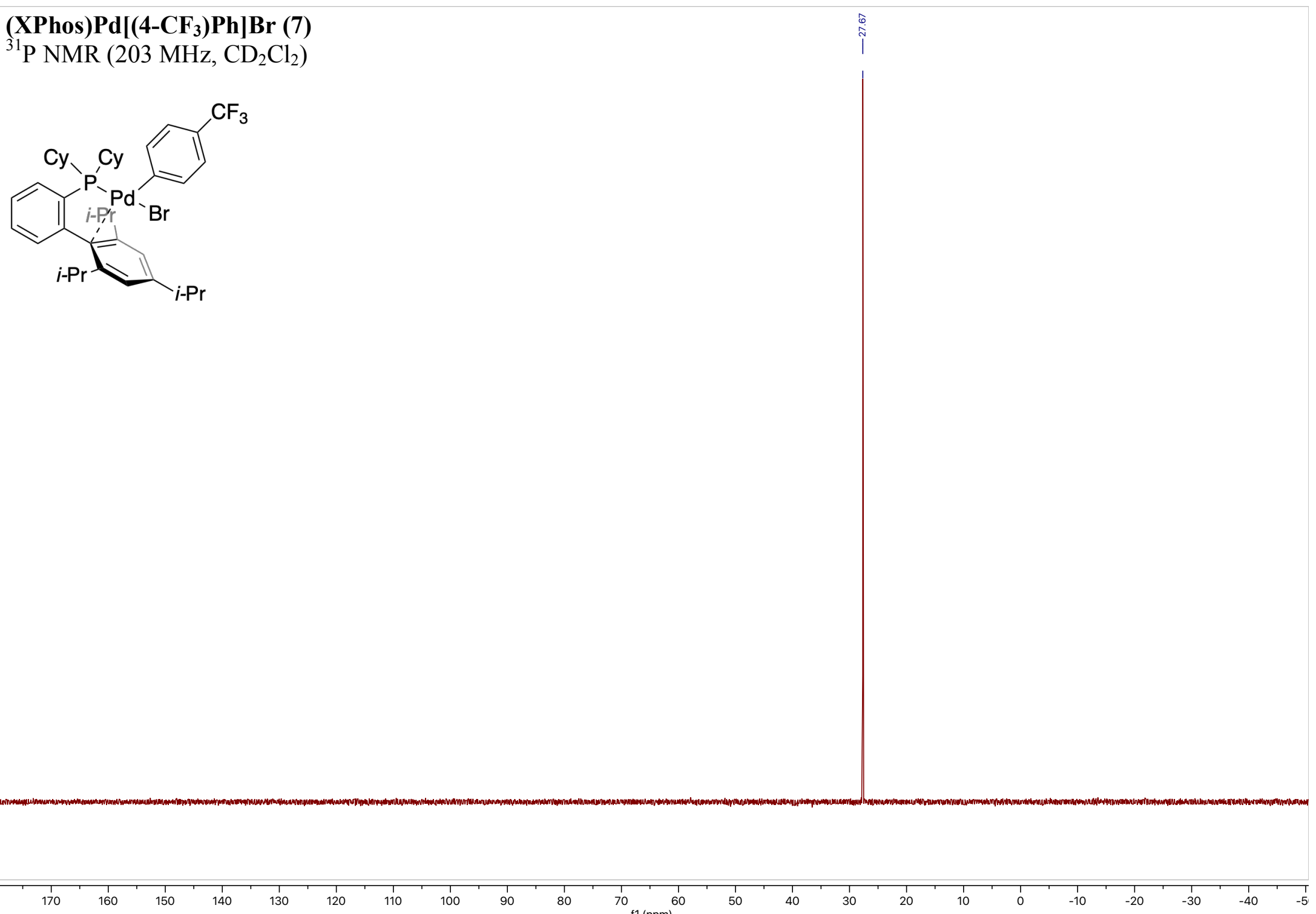

f1 (ppm) 


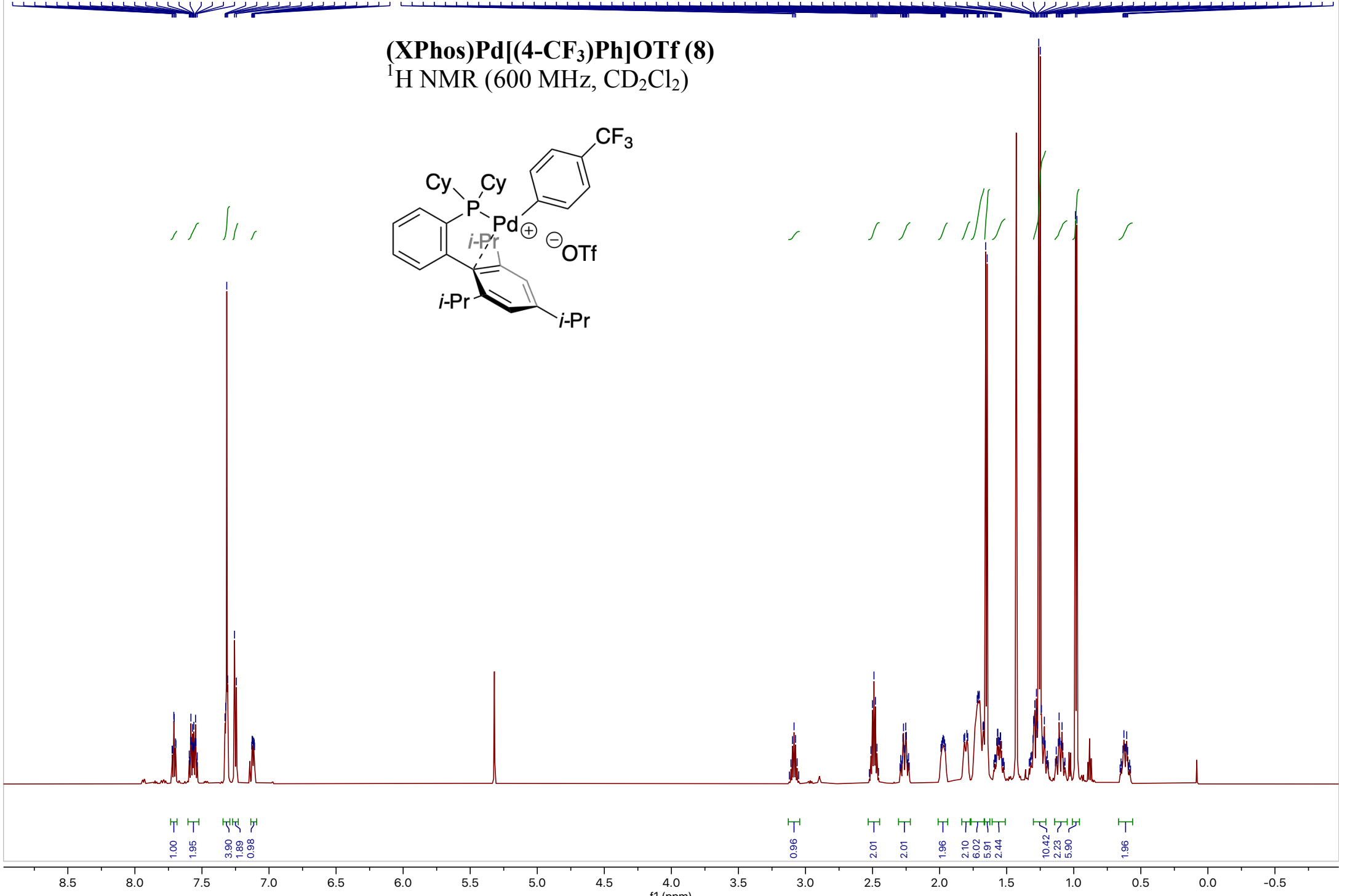




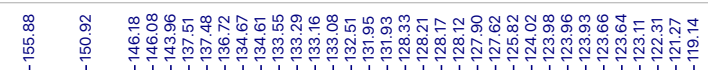

(XPhos)Pd[(4-CF 3 )Ph]OTf (8)

${ }^{13} \mathrm{C}$ NMR $\left(151 \mathrm{MHz}, \mathrm{CD}_{2} \mathrm{Cl}_{2}\right)$

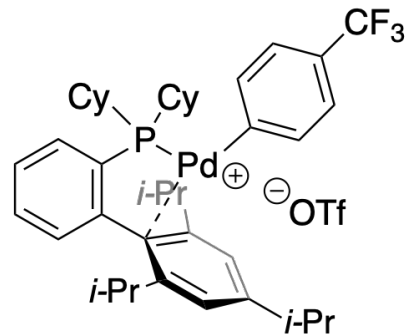

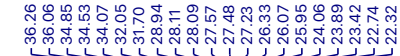

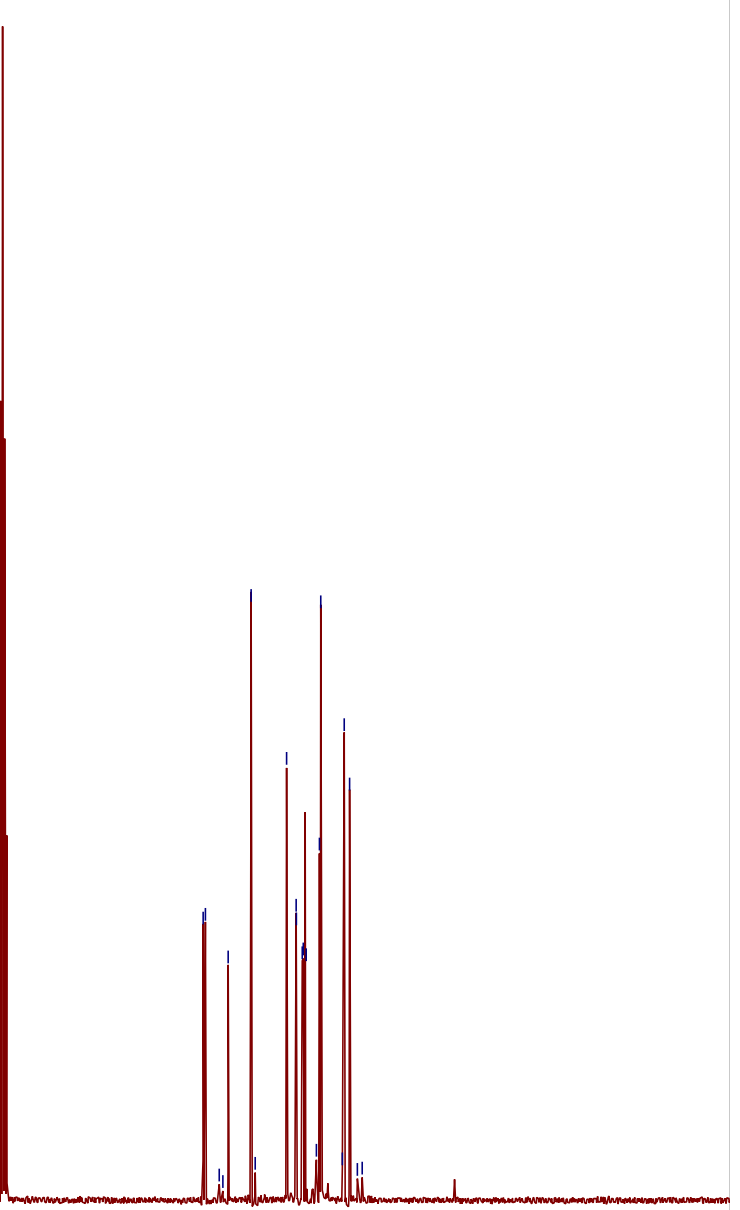

170 160 150 140 130 120 110 100 $900_{\mathrm{f1}(\mathrm{ppm})}^{80}$ 70 60 50 40 30 20 
(XPhos)Pd[(4-CF 3 )Ph]OTf (8)

${ }^{19} \mathrm{~F}$ NMR (377 MHz, $\mathrm{CDCl}_{3}$ )

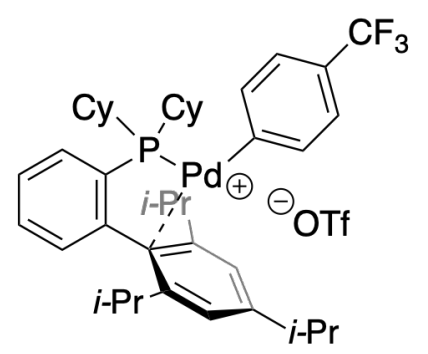

0

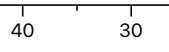
20 10
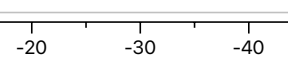
(XPhos)Pd[(4-CF 3$) P h] O T f(8)$

${ }^{31} \mathrm{P}$ NMR $\left(162 \mathrm{MHz}, \mathrm{CDCl}_{3}\right)$

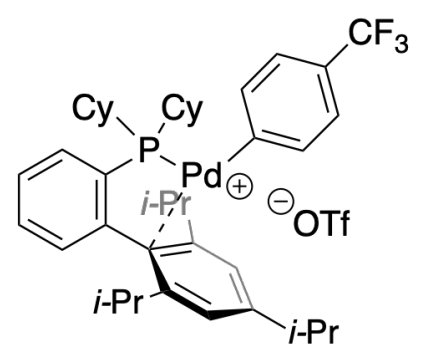

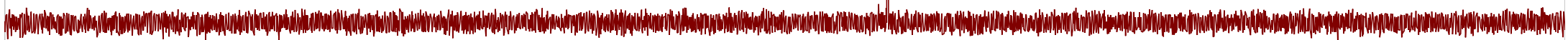




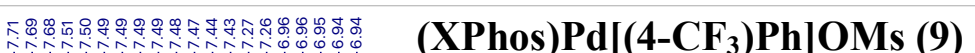

${ }^{1} \mathrm{H}$ NMR $\left(500 \mathrm{MHz}, \mathrm{CD}_{2} \mathrm{Cl}_{2}\right)$

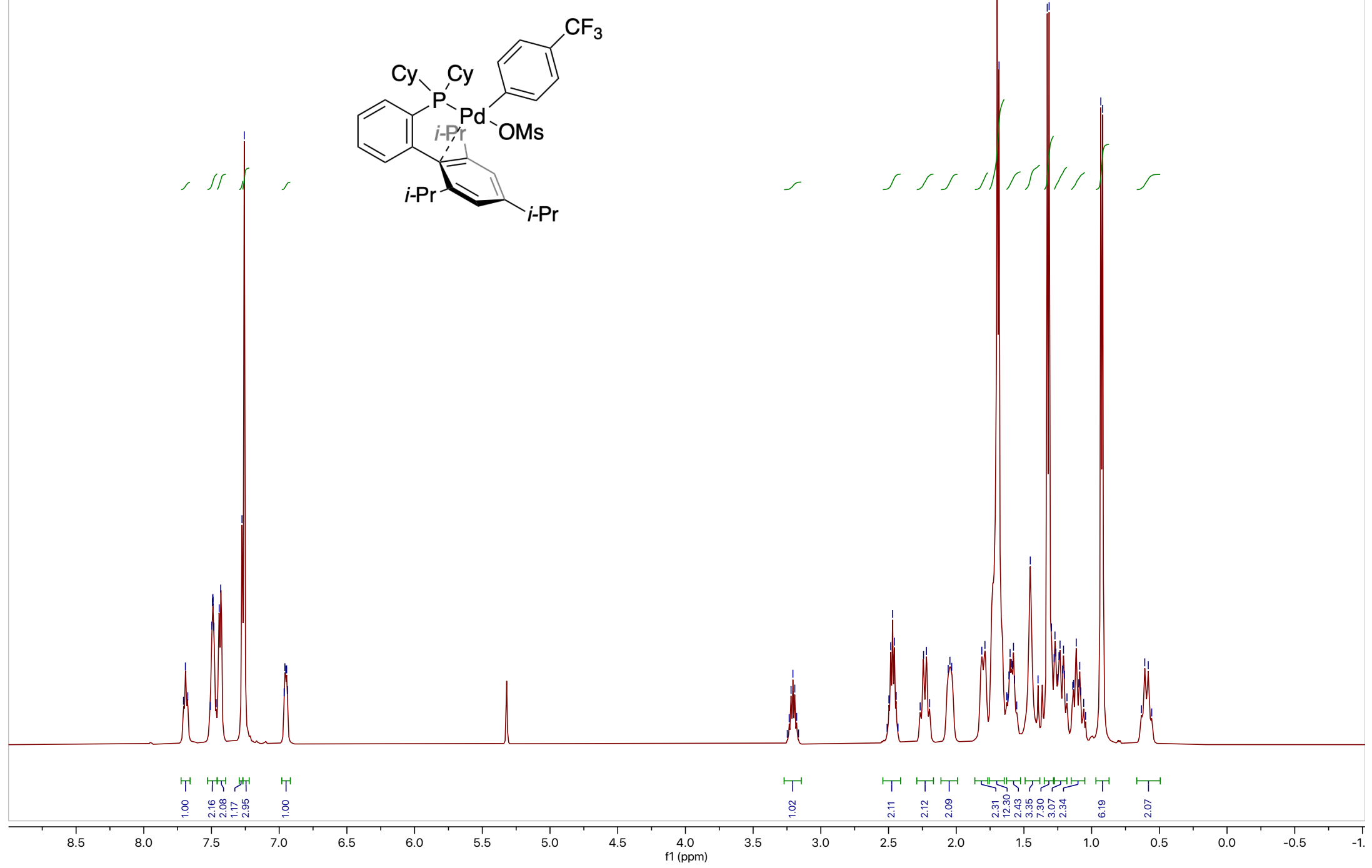


(XPhos)Pd[(4-CF 3 )Ph]OMs (9)

${ }^{13} \mathrm{C}$ NMR $\left(126 \mathrm{MHz}, \mathrm{CD}_{2} \mathrm{Cl}_{2}\right)$

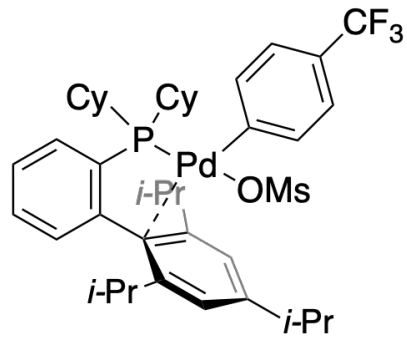

\section{$\mathrm{F}_{3}$}
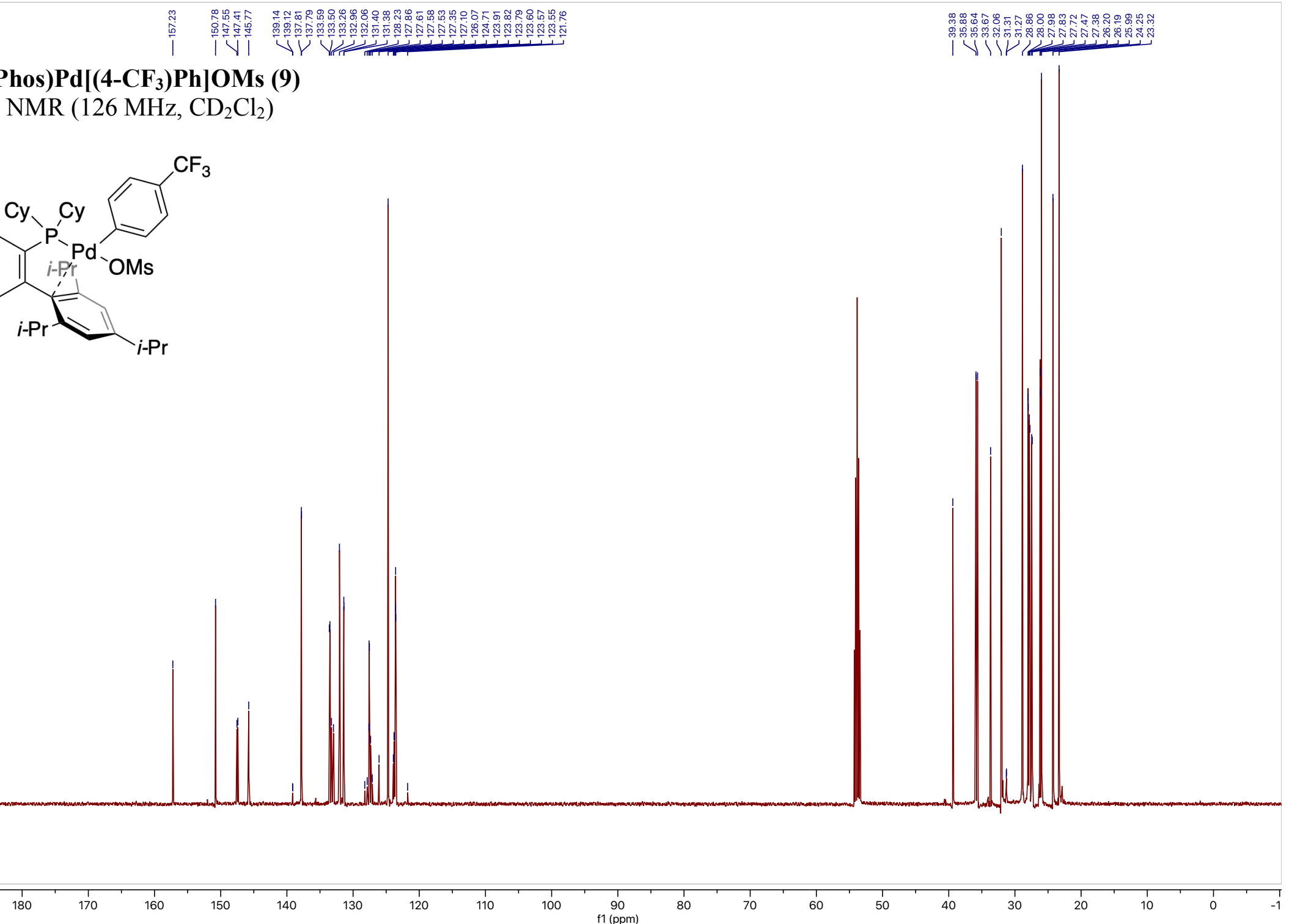

170

160

150

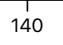

130

120
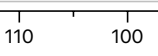

10
$11(\mathrm{ppm})$

80

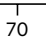

60

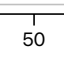

40

30

20 
(XPhos)Pd[(4-CF 3 )Ph]OMs (9)

${ }^{19} \mathrm{~F}$ NMR $\left(471 \mathrm{MHz}, \mathrm{CD}_{2} \mathrm{Cl}_{2}\right)$

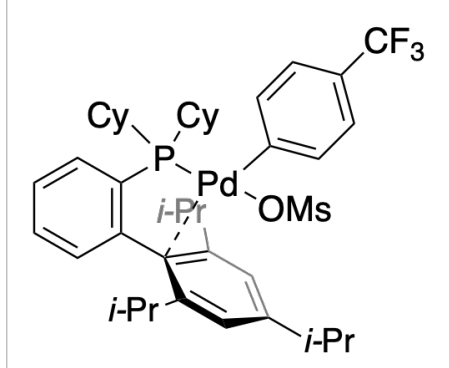

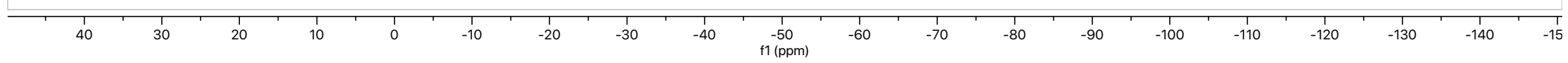


(XPhos)Pd[(4-CF 3 )Ph]OMs (9)

${ }^{31} \mathrm{P}$ NMR (203 MHz, $\mathrm{CD}_{2} \mathrm{Cl}_{2}$ )

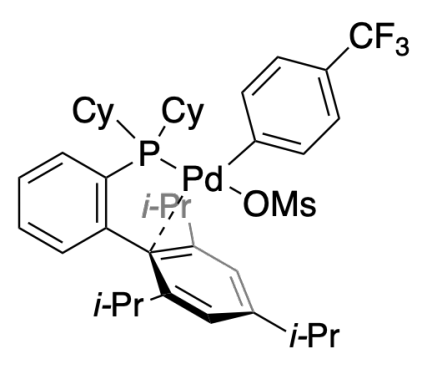


(RuPhos)Pd[(4-CF 3 )Ph]Cl (10)

${ }^{1} \mathrm{H}$ NMR (600 MHz, $\mathrm{CD}_{2} \mathrm{Cl}_{2}$ )
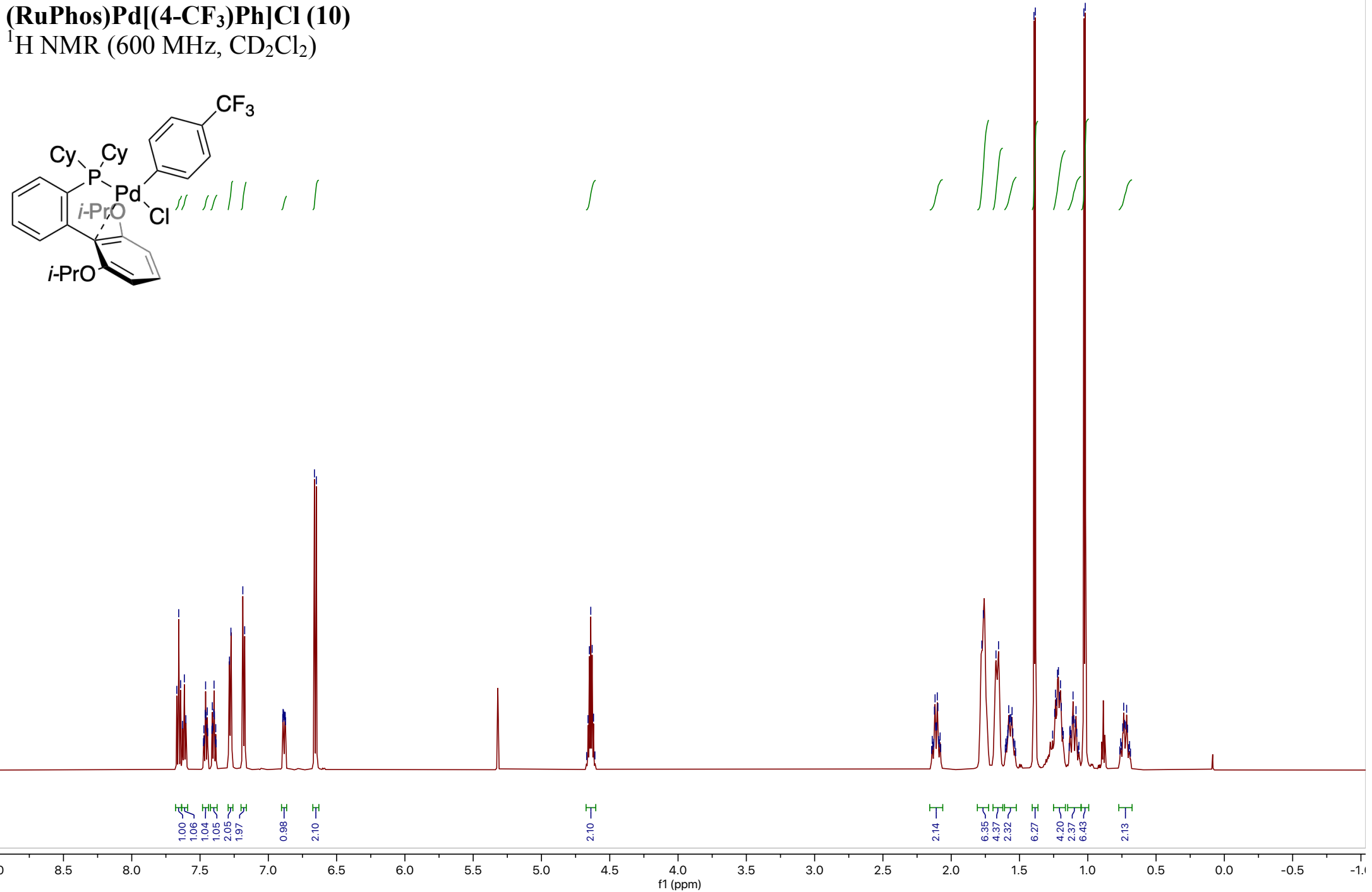


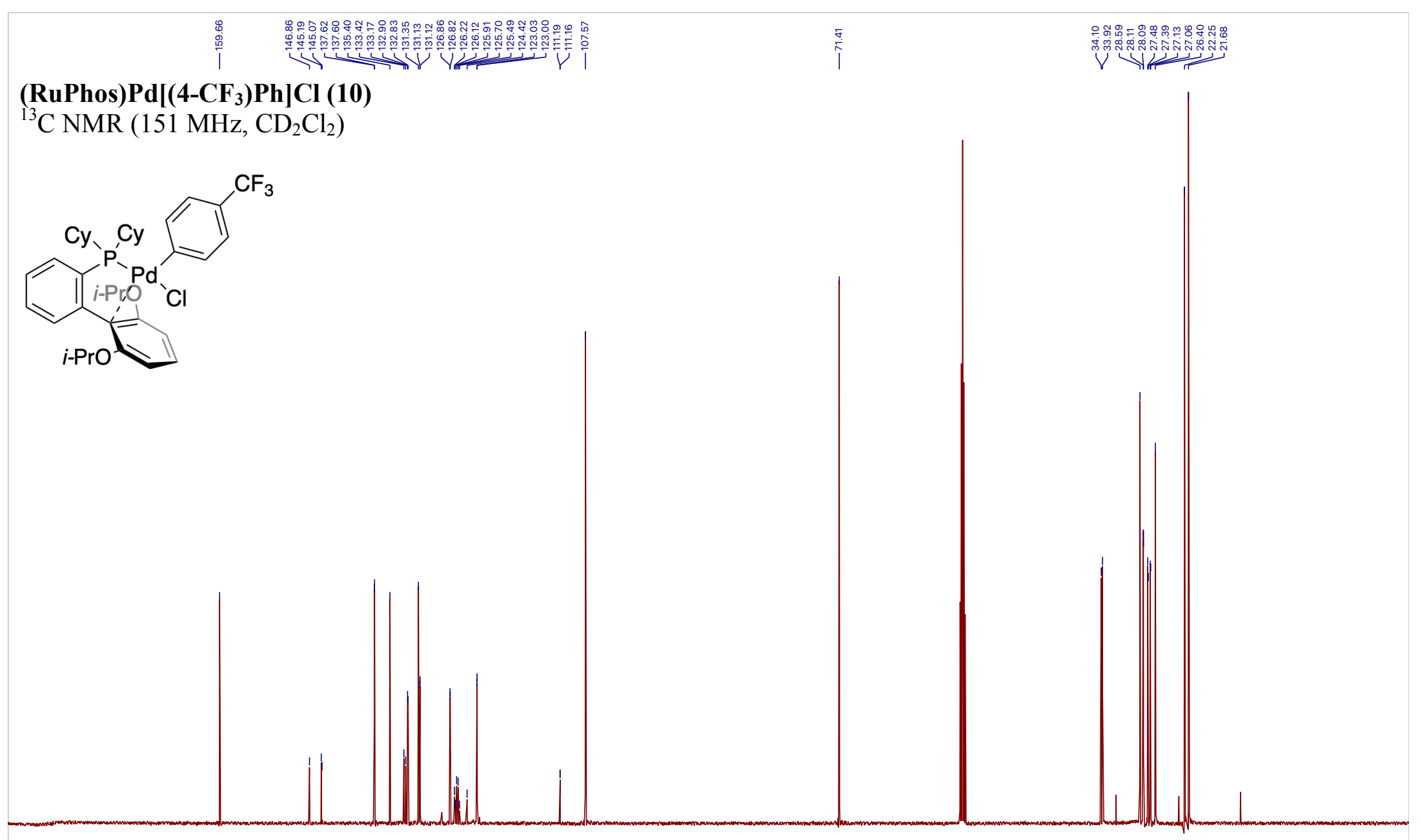


(RuPhos)Pd[(4-CF 3 )Ph]Cl (10)

${ }^{19} \mathrm{~F}$ NMR $\left(565 \mathrm{MHz}, \mathrm{CD}_{2} \mathrm{Cl}_{2}\right)$

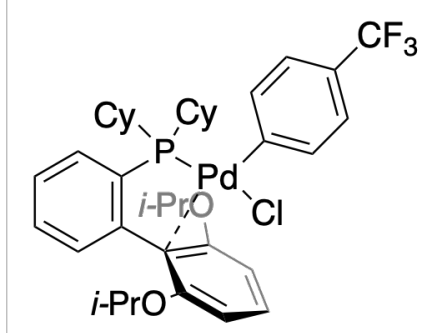

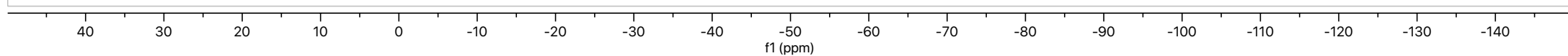


(RuPhos)Pd[(4-CF 3 )Ph]Cl (10)

${ }^{31} \mathrm{P}$ NMR (202 MHz, $\mathrm{CD}_{2} \mathrm{Cl}_{2}$ )

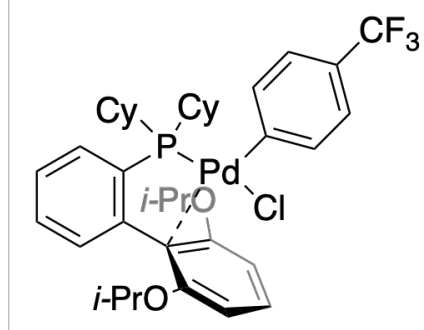

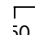

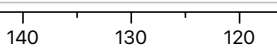
110 100 90 80 $70 \quad 60$ 50
$f 1(\mathrm{ppm})$ 40 30 20 10 


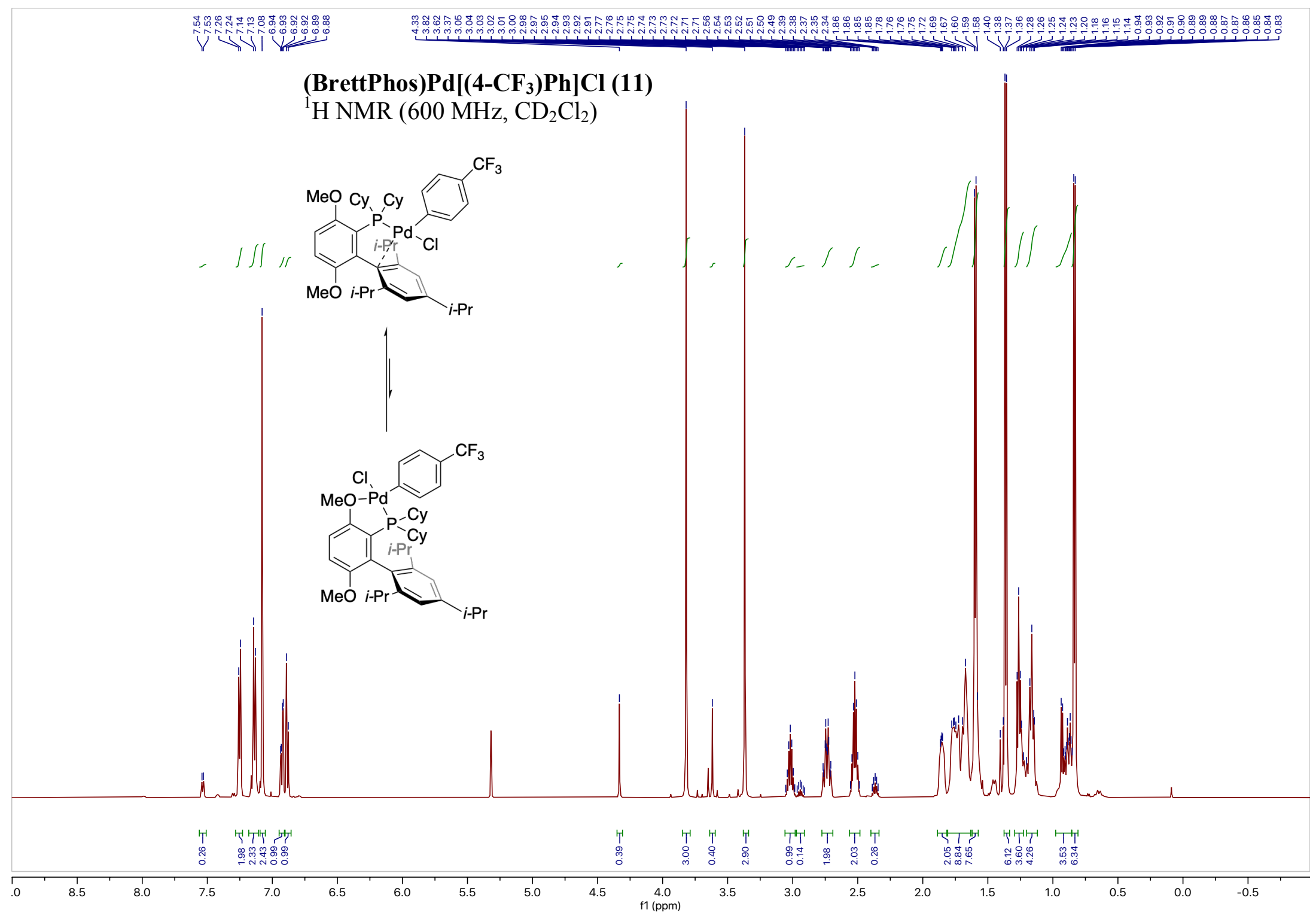




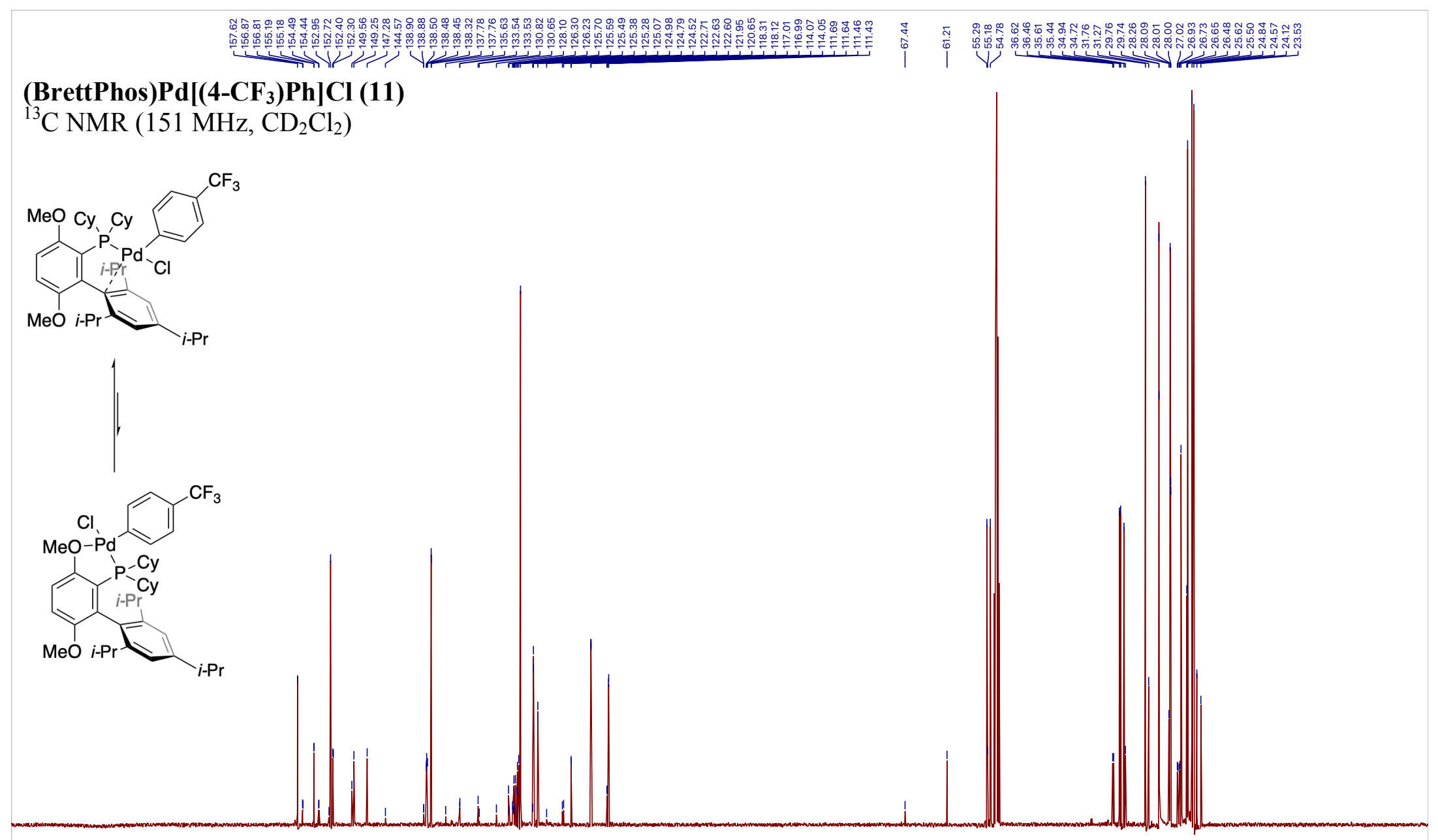


(BrettPhos)Pd[(4-CF 3$) \mathrm{Ph}] \mathrm{Cl}$ (11)

${ }^{19} \mathrm{~F}$ NMR (565 MHz, $\mathrm{CD}_{2} \mathrm{Cl}_{2}$ )
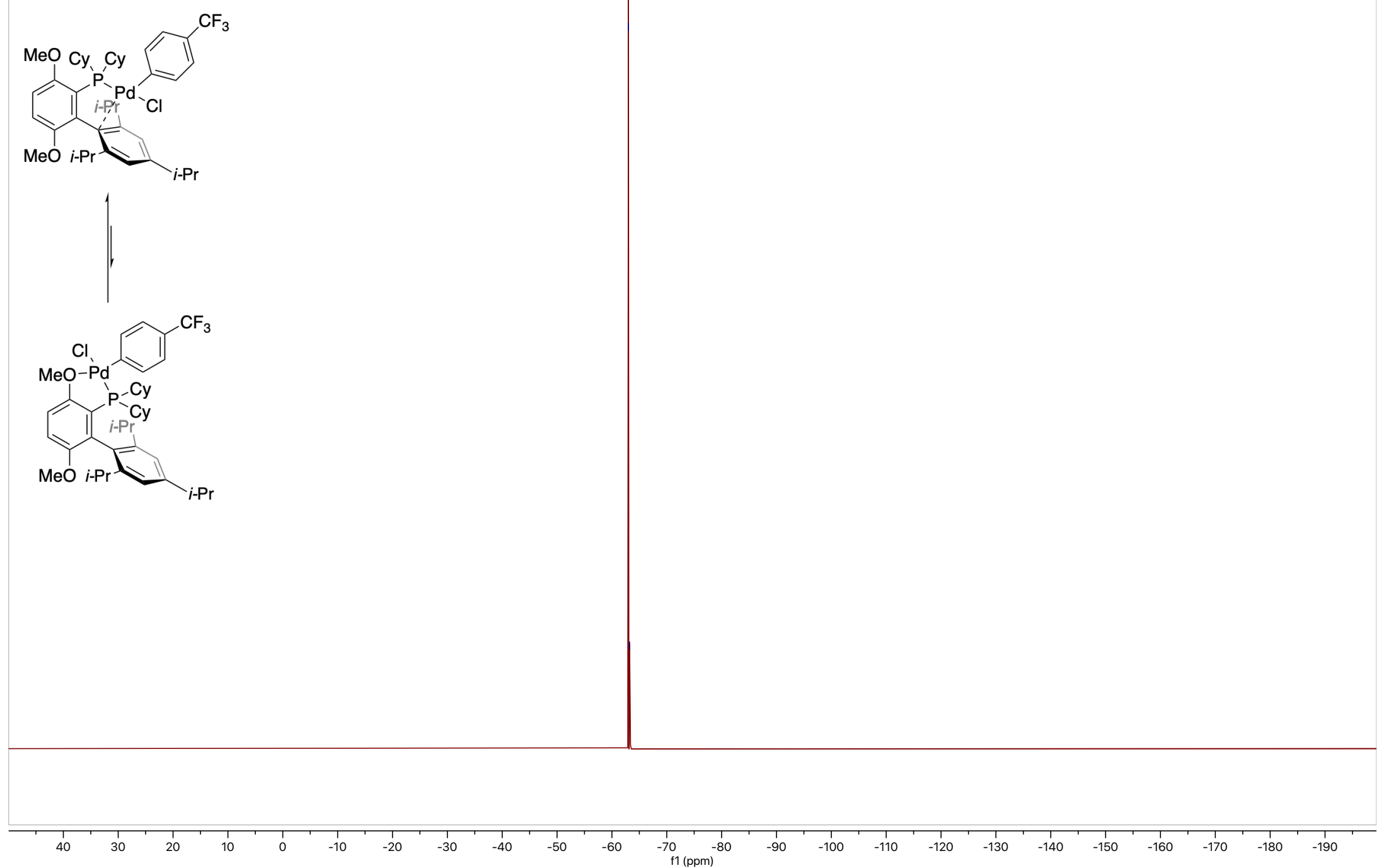
(BrettPhos)Pd[(4-CF 3$) P h] C l(11)$

${ }^{31} \mathrm{P}$ NMR (202 MHz, $\mathrm{CD}_{2} \mathrm{Cl}_{2}$ )

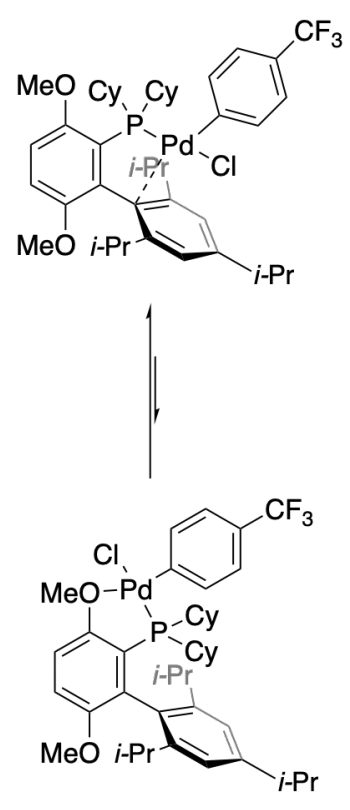

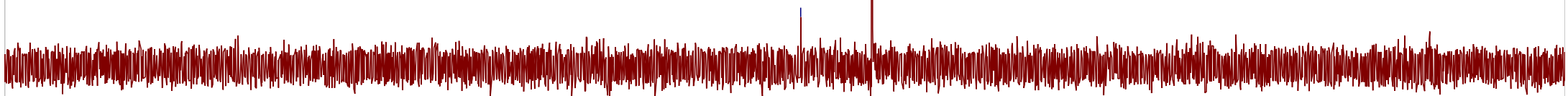

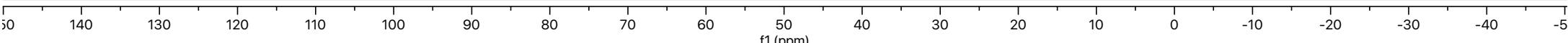




\section{(XPhos)Pd(Ph)Cl (12)}

${ }^{1} \mathrm{H}$ NMR $\left(600 \mathrm{MHz}, \mathrm{CD}_{2} \mathrm{Cl}_{2}\right)$
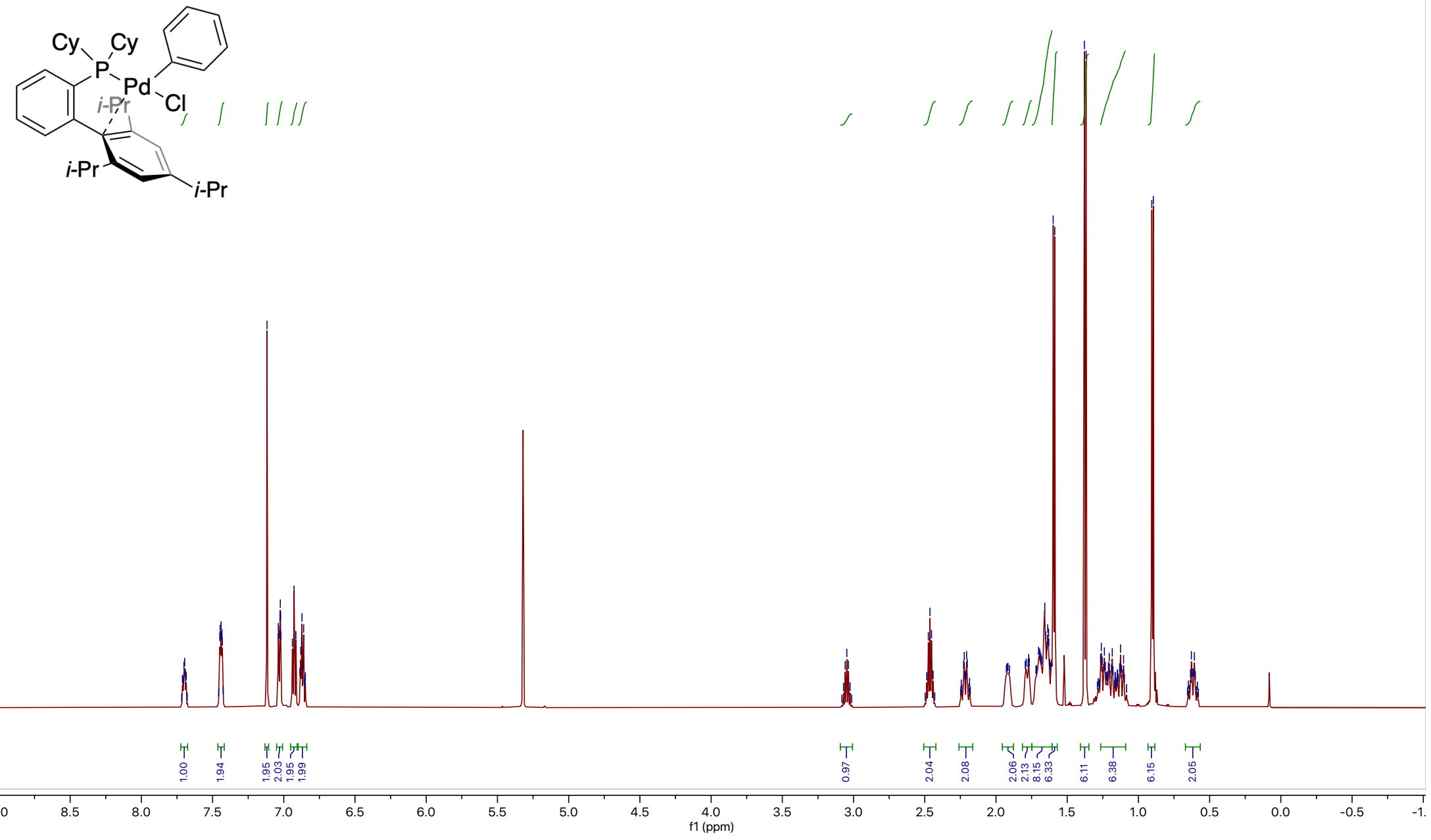
(XPhos)Pd(Ph)Cl (12)

${ }^{13} \mathrm{C}$ NMR $\left(151 \mathrm{MHz}, \mathrm{CD}_{2} \mathrm{Cl}_{2}\right.$ )
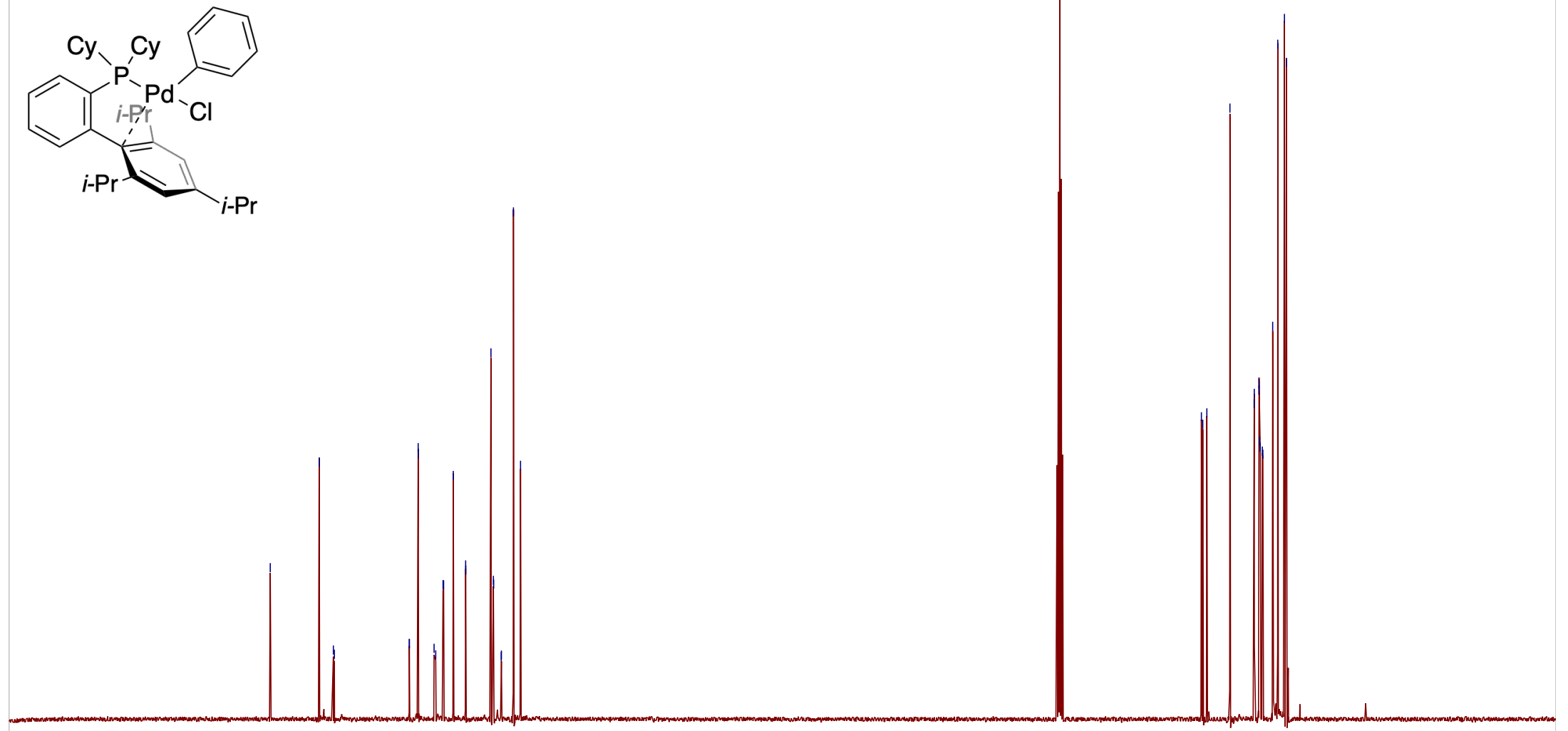

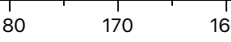
150 140 130 120 $110+100$ 90
$11(p p m)$ 80 70 60 $50 \quad 40$ 30 20 $10+0$ 
(XPhos)Pd(Ph)Cl (12)

${ }^{31} \mathrm{P}$ NMR (203 MHz, $\mathrm{CD}_{2} \mathrm{Cl}_{2}$ )

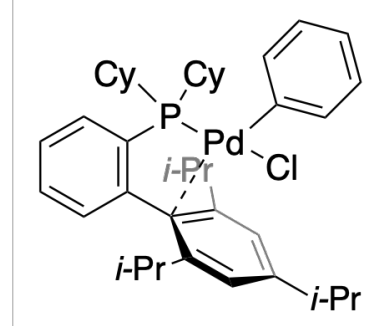

5 


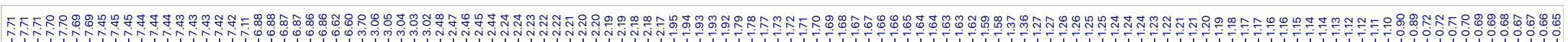
(XPhos)Pd[(4-OMe)Ph]Cl (13)

${ }^{1} \mathrm{H} \mathrm{NMR}\left(600 \mathrm{MHz}, \mathrm{CD}_{2} \mathrm{Cl}_{2}\right)$

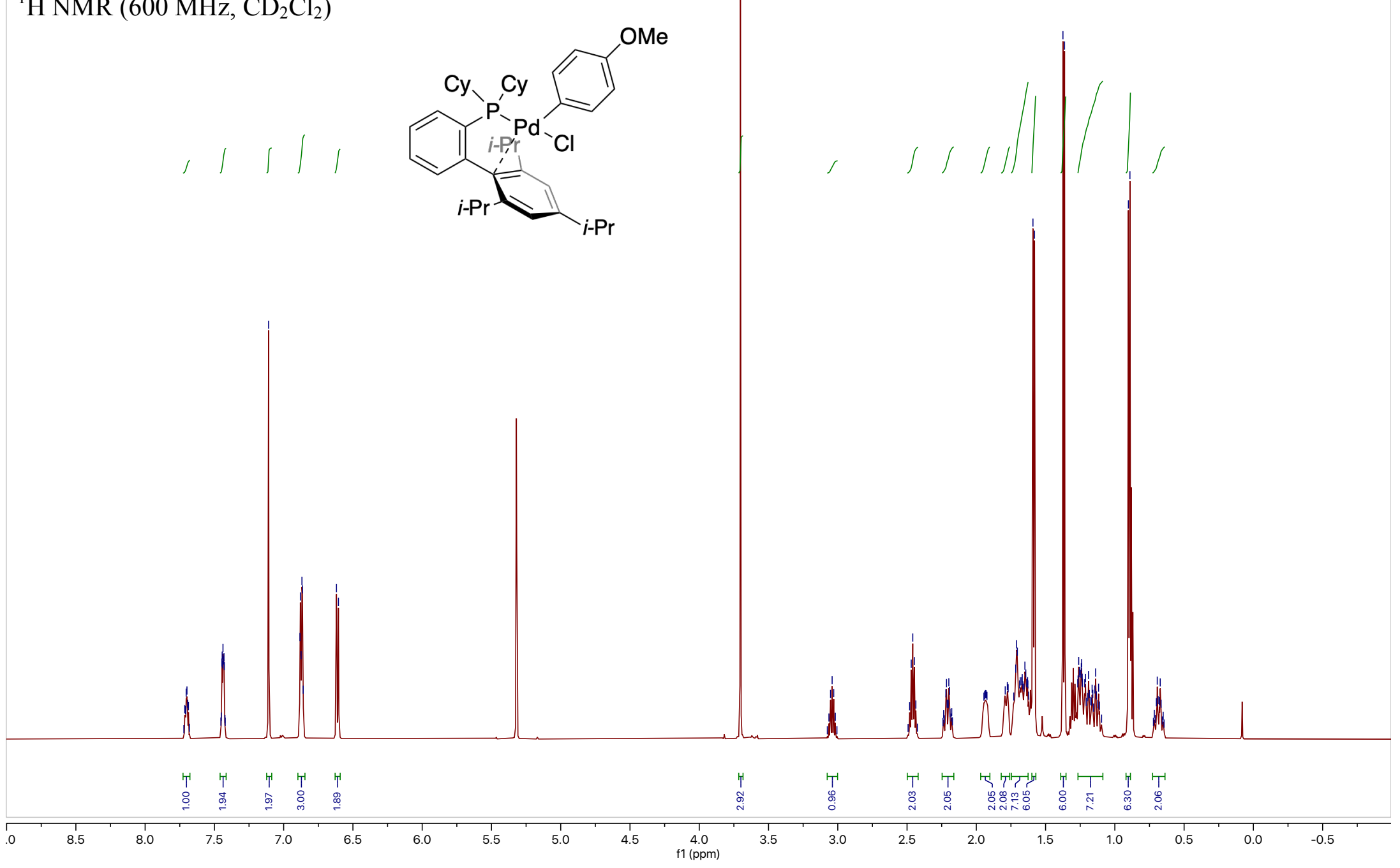


(XPhos)Pd[(4-OMe)Ph]Cl (13)

${ }^{13} \mathrm{C}$ NMR (126 MHz, $\left.\mathrm{CD}_{2} \mathrm{Cl}_{2}\right)$

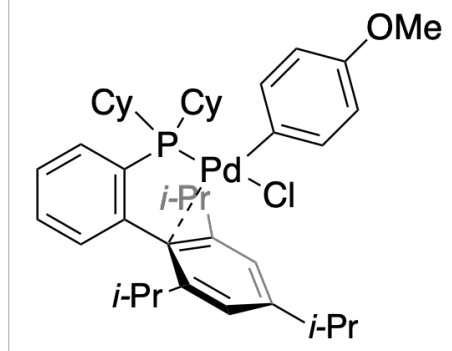


(XPhos)Pd[(4-OMe)Ph]Cl (13)

${ }^{31} \mathrm{P}$ NMR (203 MHz, $\mathrm{CD}_{2} \mathrm{Cl}_{2}$ )
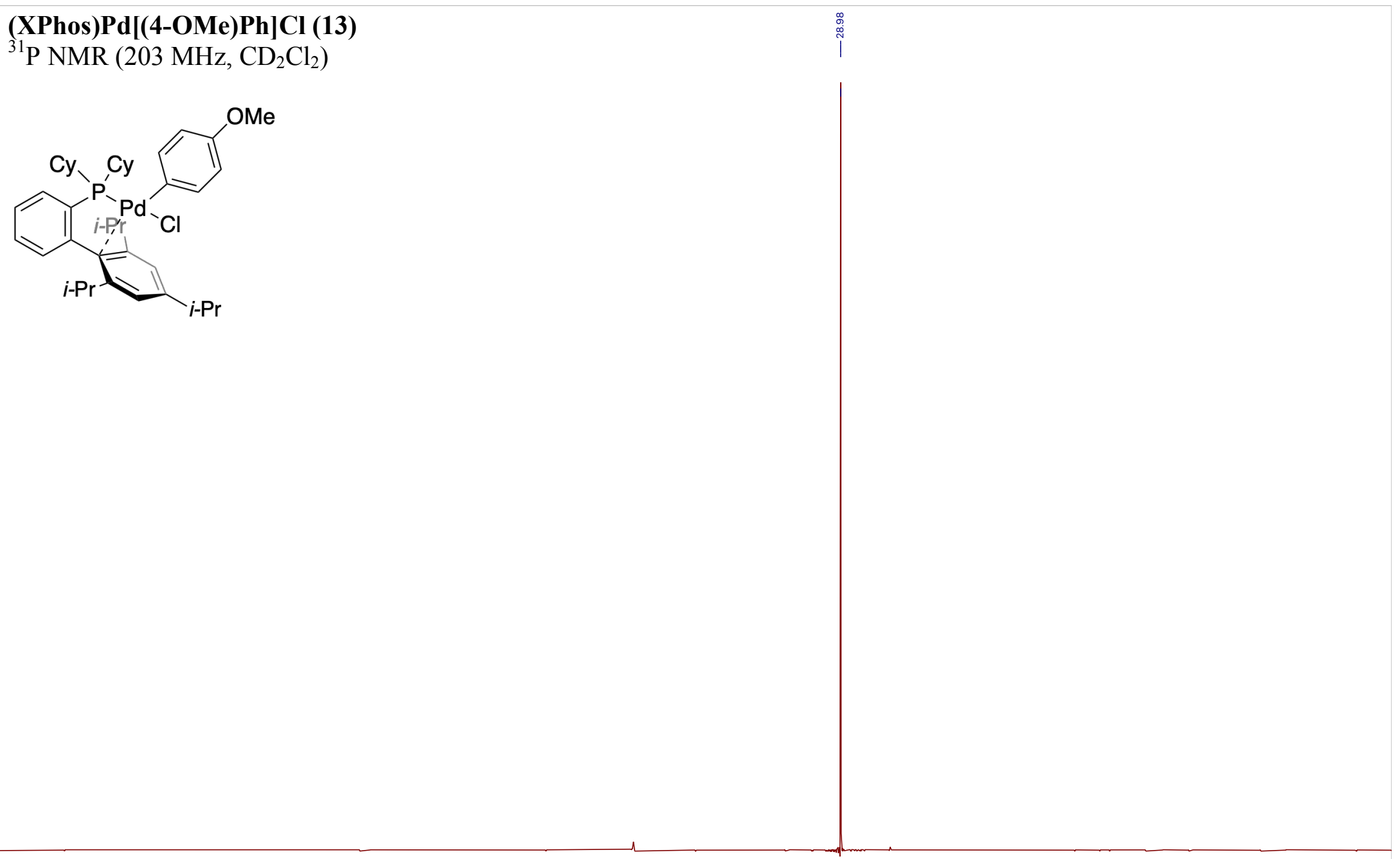

$50 \quad 140$ $130 \quad 120$ 110 100 90 80 $70 \quad 60$ 50
$\mathrm{f} 1(\mathrm{ppm})$ 


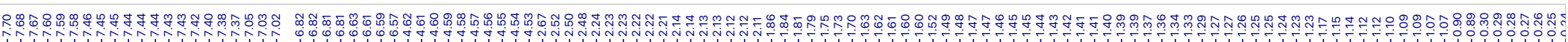

(RuPhos)Pd[(2-Me-4-CF 3 )Ph]Br (14)

${ }^{1} \mathrm{H}$ NMR $\left(600 \mathrm{MHz}, \mathrm{CD}_{2} \mathrm{Cl}_{2}\right.$ )

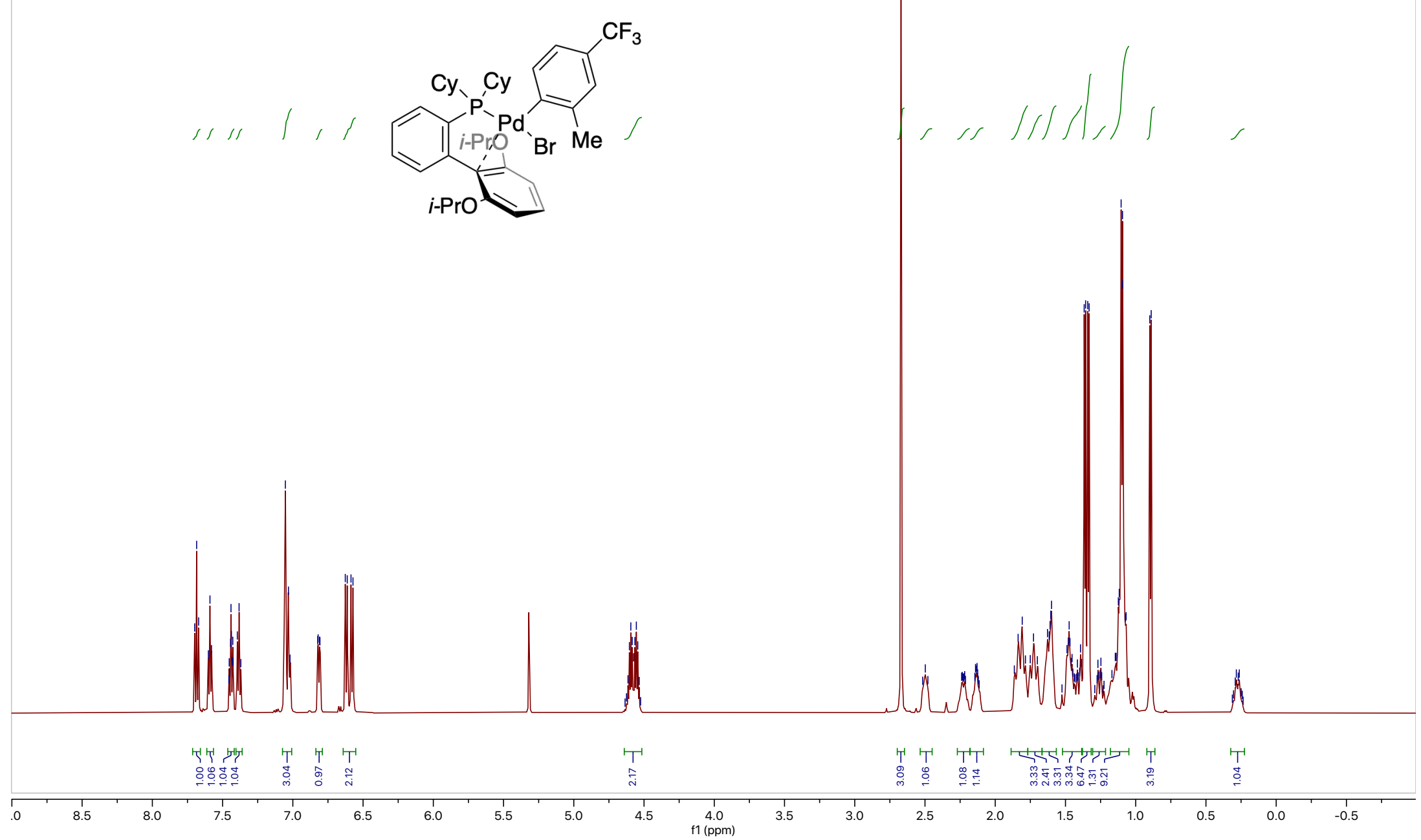




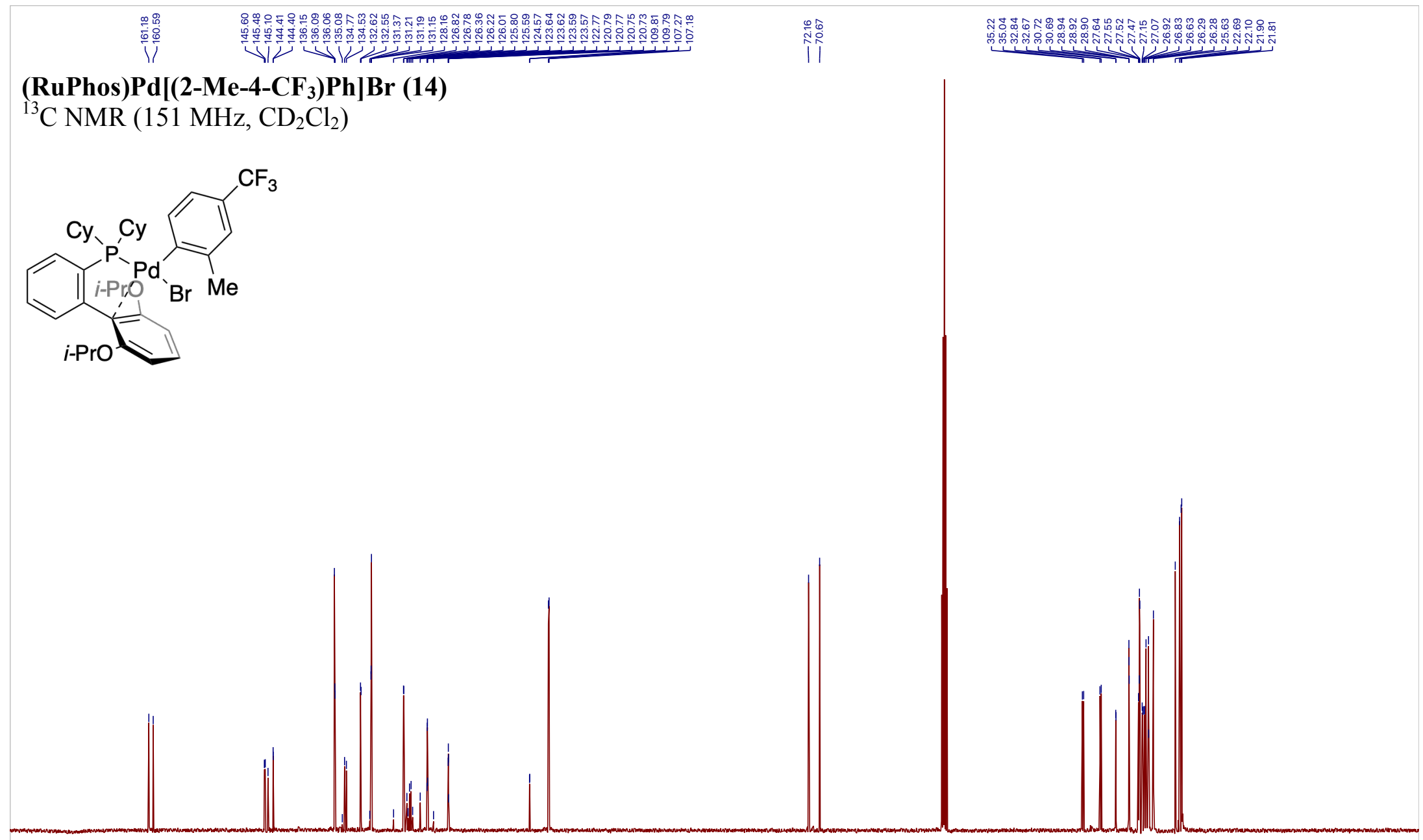

170

160 150 140 130 120 $110 \quad 100$ $90_{\mathrm{f1}(\mathrm{ppm})} 80$ 70 60 50 $40 \quad 30$ 10 
(RuPhos)Pd[(2-Me-4-CF 3$) P h] B r(14)$

${ }^{9} \mathrm{~F}$ NMR (565 MHz, $\mathrm{CD}_{2} \mathrm{Cl}_{2}$ )
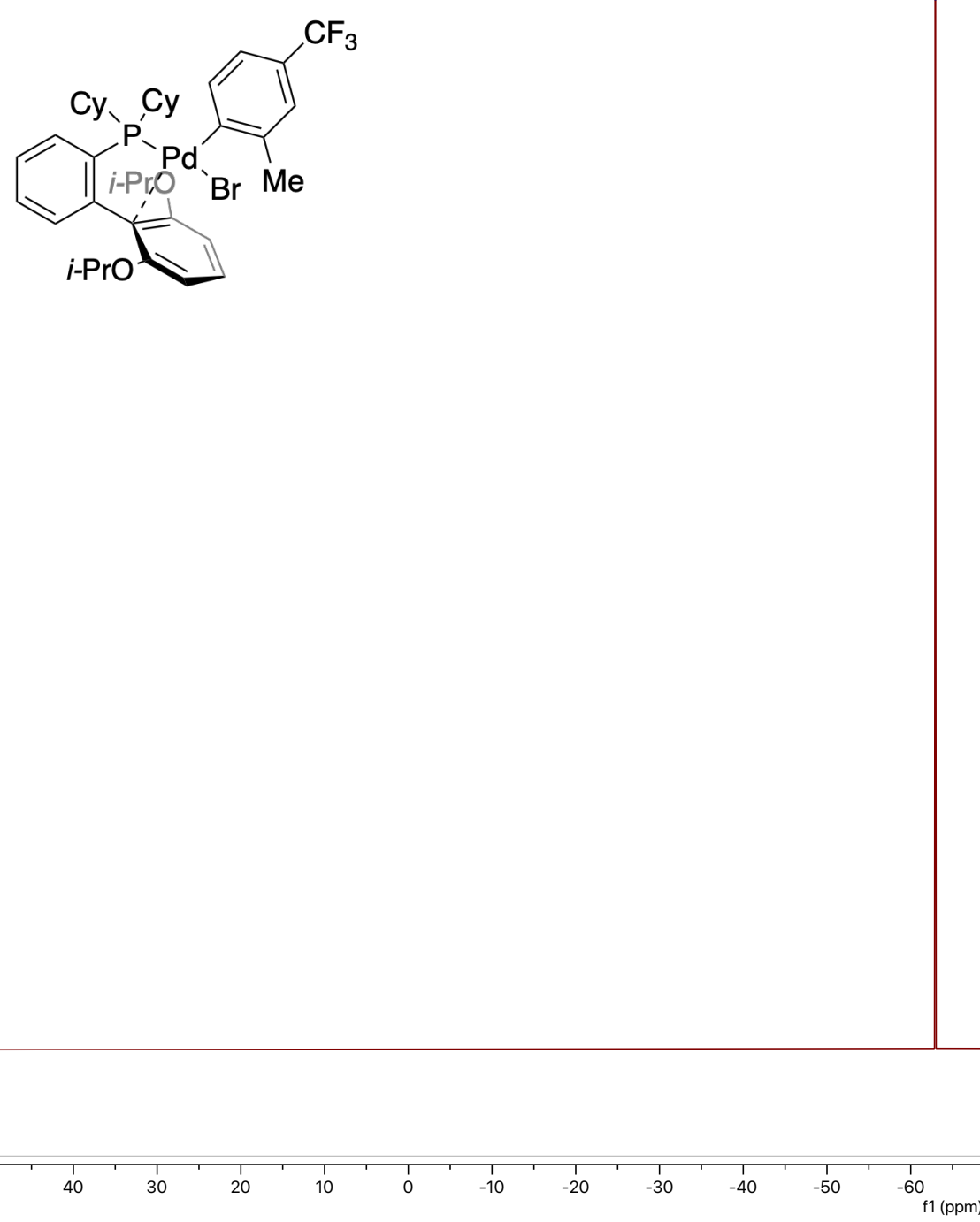
(RuPhos)Pd[(2-Me-4-CF 3 )Ph]Br (14)

${ }^{31} \mathrm{P}$ NMR (203 MHz, $\mathrm{CD}_{2} \mathrm{Cl}_{2}$ )

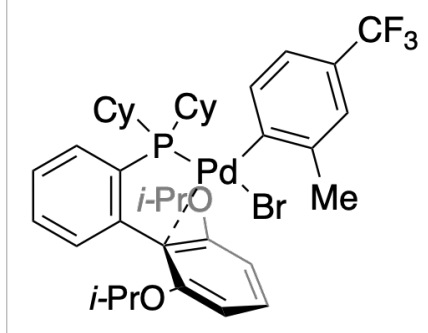




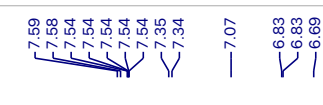

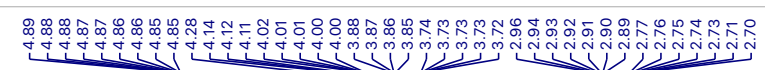
-

(S)-N-((2-0xo-3-(4-(3-oxomorpholino)phenyl)oxazolidin-5-yl)methyl)-5(2,4,6-triisopropylphenyl)thiophene-2-carboxamide (15)

${ }^{1} \mathrm{H}$ NMR (600 MHz, $\mathrm{CD}_{2} \mathrm{Cl}_{2}$ )<smiles>CCCc1cc(C(C)C)c(-c2ccc(C(=O)NCC3CN(c4ccc(N5CCOCC5=O)cc4)C(=O)O3)s2)c(C(C)C)c1</smiles>

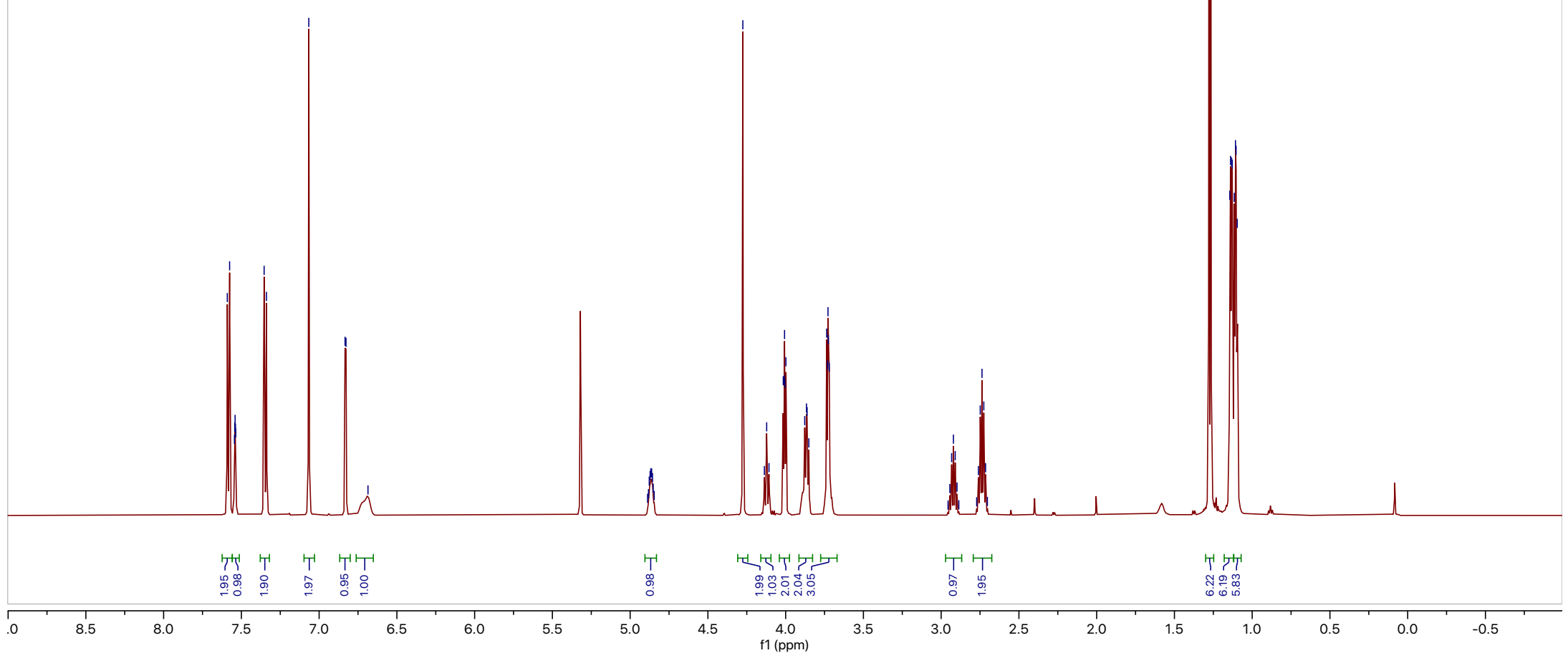




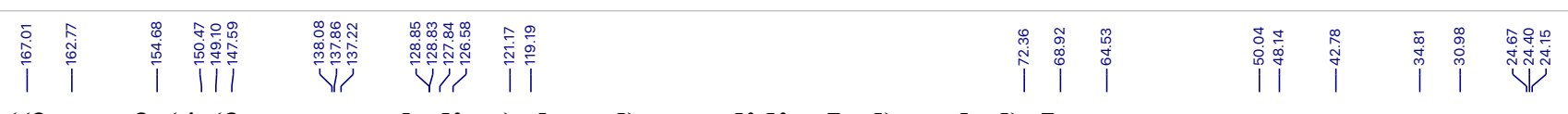

(S)-N-((2-oxo-3-(4-(3-oxomorpholino)phenyl)oxazolidin-5-yl)methyl)-5(2,4,6-triisopropylphenyl)thiophene-2-carboxamide (15)

${ }^{13} \mathrm{C}$ NMR (151 MHz, $\mathrm{CD}_{2} \mathrm{Cl}_{2}$ )
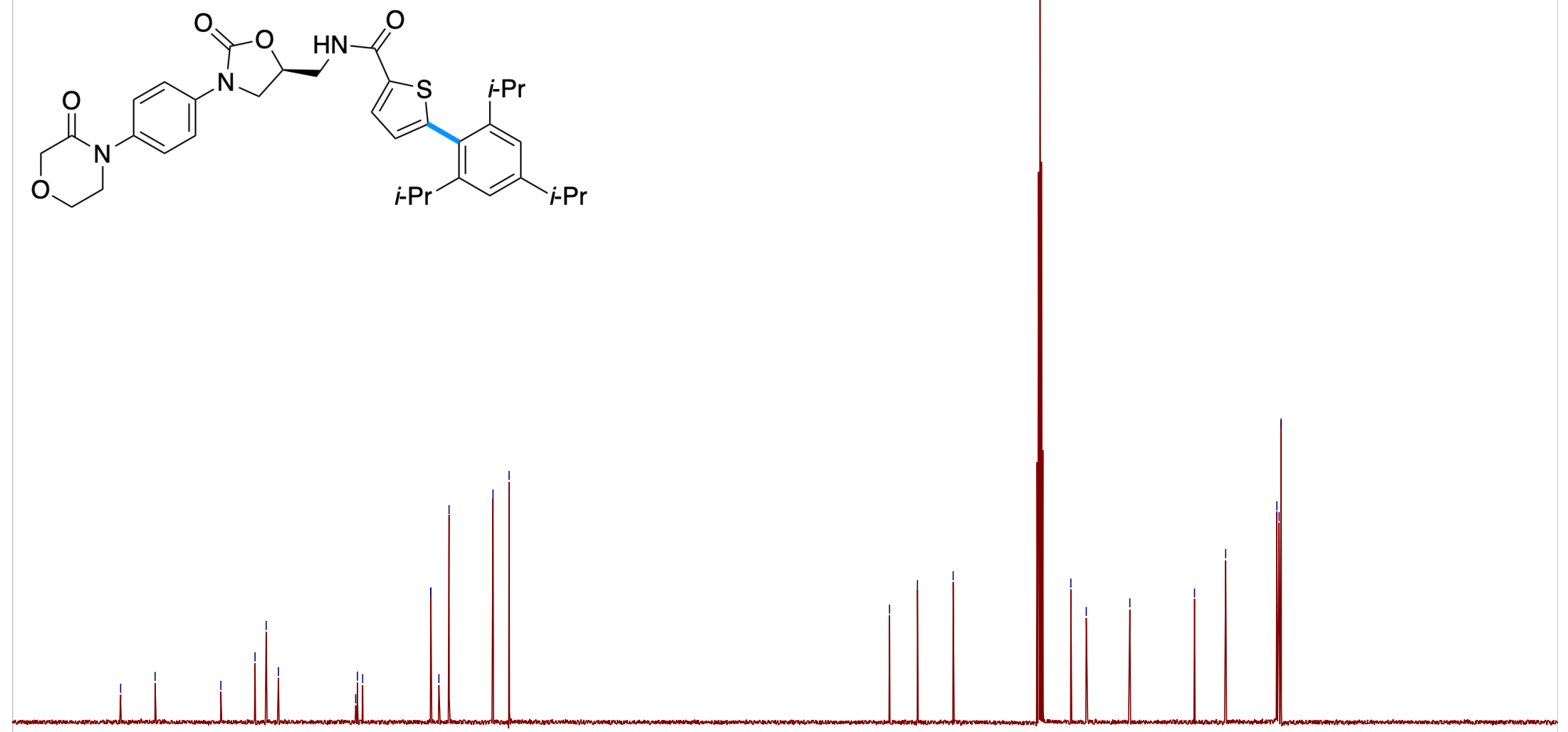


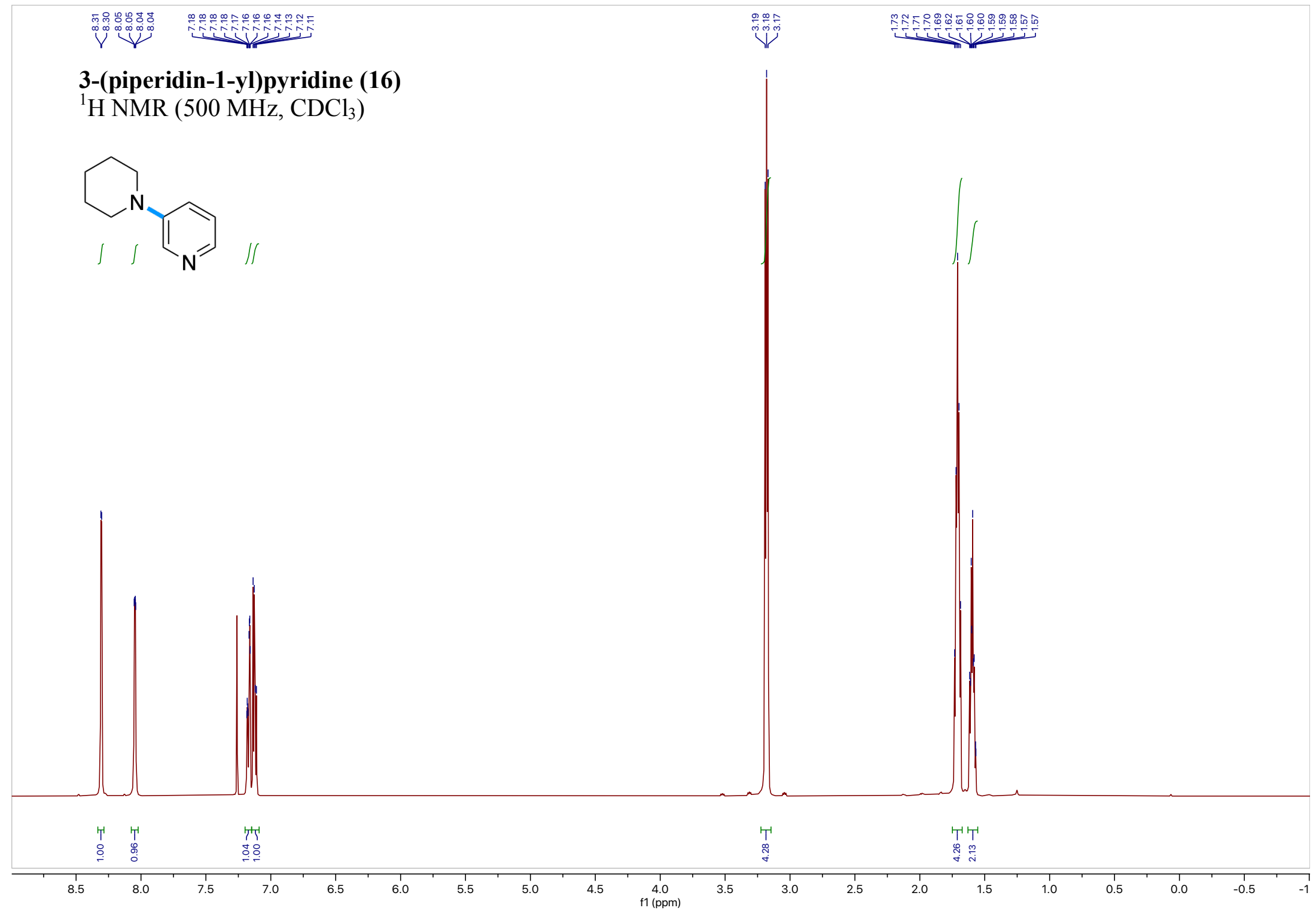




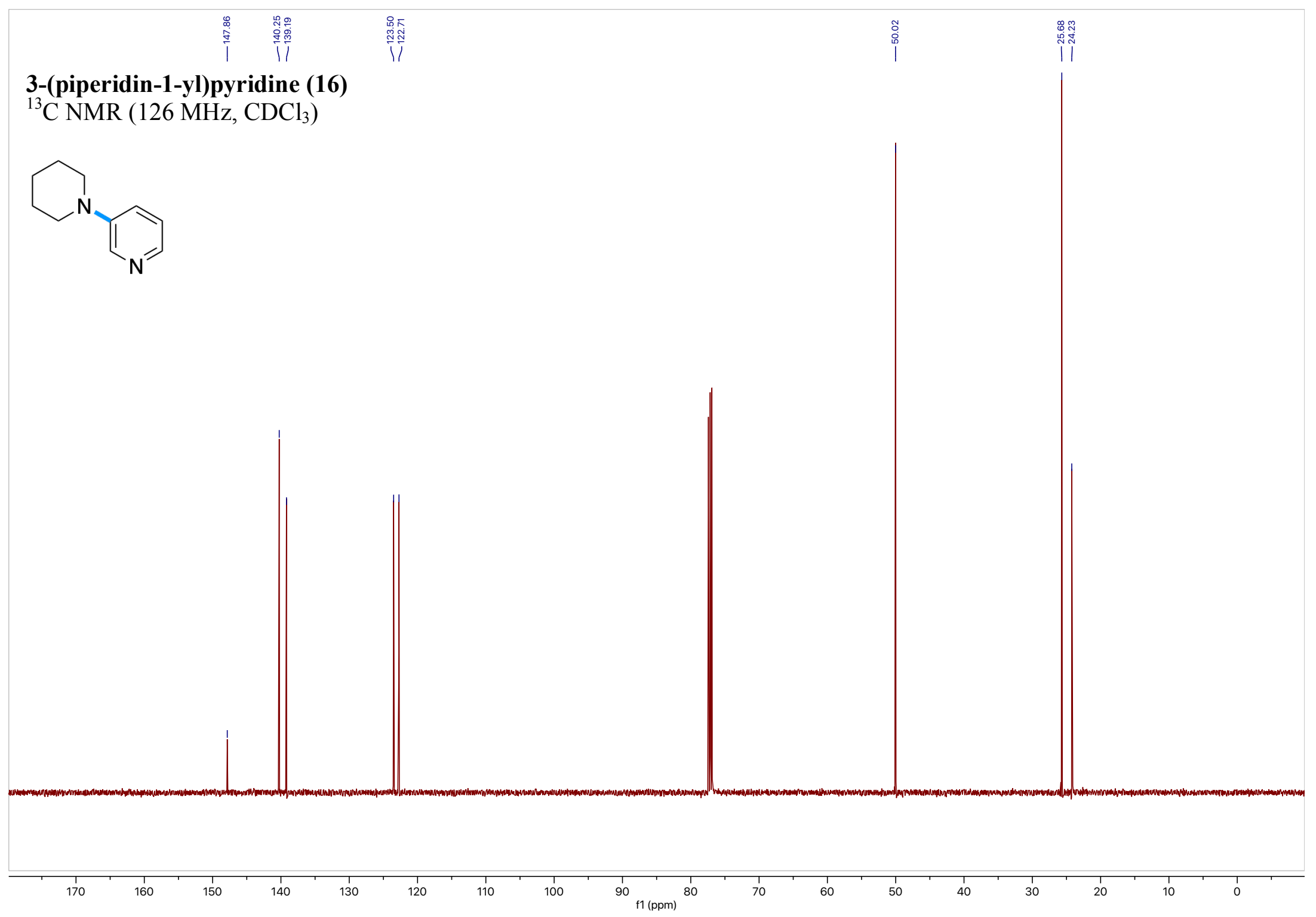

S170 